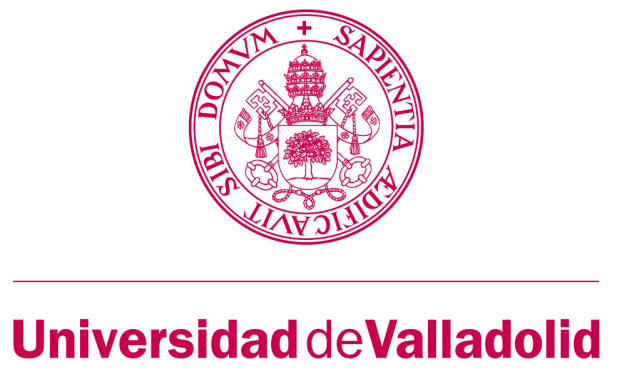

TESIS DOCTORAL

\title{
TOPOLOGIES OF CONTINUITY FOR \\ CARATHÉODORY DIFFERENTIAL EQUATIONS \\ WITH APPLICATIONS IN \\ NON-AUTONOMOUS DYNAMICS
}

Presentada por Iacopo Paolo Longo para optar

al grado de Doctor por la Universidad de Valladolid

Dirigida por

Sylvia Novo y Rafael Obaya 

Certificamos que la presente memoria ha sido realizada por Iacopo Paolo Longo bajo nuestra dirección en el Departamento de Matemática Aplicada de la Universidad de Valladolid.

Valladolid, a 25 de Julio 2018.

Fdo.: Dra Sylvia Novo Martín

Dr. Rafael Obaya García 

Ai miei genitori, con tutto il mio amore. 

I would like to truly thank Rafael Obaya and Sylvia Novo, not only for helping me to complete this dissertation, but above all for the privilege of working with them in the past years, in which I have grown as a mathematician and as a person. I will never forget that.

From the bottom of my heart, I thank my family for being always so supportive with me. Despite living so far, I have always felt your warmly comforting vicinity.

A special thank goes to all the members of the European Innovative Training Network CRITICS, especially to Jeroen Lamb and to Martin Rasmussen who created such an ambitious group of incredibly talented people (I feel very lucky to be part of) and who were always very supportive and helpful with me, and also to all the young researchers with whom I have shared three unforgettable years.

Similarly, I would like to thank all the members of the Department of Applied Mathematics of the University of Valladolid for the valuable time spent together every day.

Last, but not the least, I would like to thank all the people from all over the world that made my life special in the last three years; the many people that I had the possibility to know, to care of and that cared of me; those that left and those that will never leave. Among them, a special thank goes to Laura and to Elisabetta. 


\section{Contents}

$\begin{array}{ll}\text { Introduction } & 1\end{array}$

1 Preliminaries 9

1.1 Carathéodory differential equations . . . . . . . . . . . 10

1.2 Basic notions of non-autonomous dynamics . . . . . . . . . . . . . . . 13

1.2.1 Processes and skew product flows . . . . . . . . . . . 13

1.2.2 Exponential dichotomy and dichotomy spectrum . . . . . 16

2 Spaces and Topologies $\quad 19$

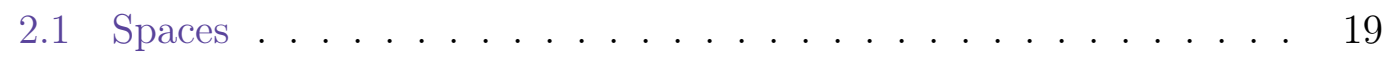

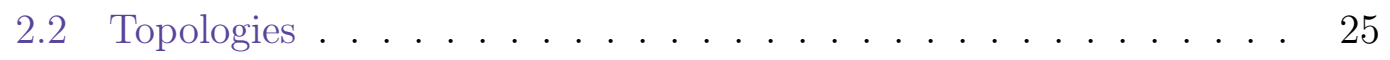

2.3 The $m$-bounds and the l-bounds . . . . . . . . . . . . . . . . 28

2.4 Topological closure . . . . . . . . . . . . . . . . . . . . . . . 32

2.5 Equivalence of the topologies . . . . . . . . . . . . . . . . . . . . . 40

2.6 Relative compactness in $\mathfrak{L C}_{p} \ldots \ldots \ldots$. . . . . . . . . 46

2.6.1 Strong topologies . . . . . . . . . . . . . . . . . . . 46

2.6 .2 Weak Topologies ... . . . . . . . . . . 50

2.7 Continuity of the time translations . . . . . . . . . . . 52

3 Continuity of the flow for Carathéodory ODEs 59

3.1 Continuity of the flow . . . . . . . . . . . . . . 60

3.2 Linearized skew-product flows . . . . . . . . . . . . . . . . . . 74

3.3 Exponential dichotomy and dichotomy spectrum . . . . . . . . . . 82

3.4 More applications . . . . . . . . . . . . . . . . . . 87

3.4.1 Existence of solutions for a differential problem in $\mathfrak{W} \Theta \mathfrak{C} .87$

3.4.2 Digitization of a non-autonomous control system . . . . . . 90

3.4.3 Carathéodory compartmental systems . . . . . . . . . 93

4 Pullback and global attractors for Carathéodory ODEs $\quad 97$

4.1 Preliminary definitions and results . . . . . . . . . . . . . 98

4.2 Pullback attractors and the skew-product flow . . . . . . . . . . . 102

4.3 Comparison results for Carathéodory ODEs . . . . . . . . . . . 105

4.3.1 Comparison with a scalar Carathéodory linear equation . . 108

4.3.2 Comparison with a Carathéodory linear system . . . . . . 117 
5 Continuity of the semiflow for Carathéodory DDEs 123

5.1 Preliminaries . . . . . . . . . . . . . . . . . . . . 124

5.2 Spaces and topologies revised . . . . . . . . . . . . . . . . . . . . . . . . 125

5.3 Continuity of the semiflow . . . . . . . . . . . . . . 136

5.3.1 Continuity with respect to $\mathcal{T}_{B}$ and $\mathcal{T}_{D} \ldots \ldots \ldots$

5.3.2 Continuity with respect to $\mathcal{T}_{\Theta B}, \mathcal{T}_{\Theta D}$ and $\sigma_{\Theta D} \ldots \ldots . .140$

$\begin{array}{lr}\text { Conclusions } & 149\end{array}$

$\begin{array}{ll}\text { Summary in Spanish } & 151\end{array}$

$\begin{array}{lr}\text { Bibliography } & 161\end{array}$ 


\section{Introduction}

A basic exercise of Calculus is to prove that for any $f: D \subset \mathbb{R} \times \mathbb{R}^{N} \rightarrow \mathbb{R}^{N}$, continuous, the Cauchy problem

$$
\dot{x}=f(t, x), \quad x\left(t_{0}\right)=x_{0},
$$

is equivalent to the integral problem

$$
x(t)=x_{0}+\int_{t_{0}}^{t} f(s, x(s)) d s .
$$

However, it is straightforward to notice that, due to Lebesgue theory, the formula in (0.2) is meaningful for a broader class of functions than just the continuous ones. In other words, given $I \subset \mathbb{R}$ so that $t_{0} \in I$, if one looks for a function $x: I \subset \mathbb{R} \rightarrow \mathbb{R}^{N}$, not necessarily continuously differentiable, satisfying (0.2) in its interval of definition, then one can relax the assumptions on $f$ to the sole local integrability. The Greek and cosmopolitan mathematician Constantin Carathéodory proved in [15] that under the assumptions

- $f$ is Borel measurable,

- for every compact set $K \subset \mathbb{R}^{N}$ there exists a real-valued function $m^{K} \in L_{l o c}^{1}$ such that for almost every $t \in \mathbb{R}$, one has

$$
|f(t, x)| \leq m^{K}(t) \quad \text { for all } x \in K,
$$

- for almost every $t \in \mathbb{R}, f(t, \cdot)$ is continuous,

any problem like (0.1) admits a solution in an extended sense, that is, an absolutely continuous function defined on an interval $I \subset \mathbb{R}$ containing $t_{0}$, so that (0.1) holds almost everywhere in $I$ or, equivalently, (0.2) holds for all $t \in I$.

Such a class of differential problems has been named after Carathéodory, and contributed to enlarge the field of applicability of differential equations in both theoretical and applied studies. As a matter of fact, the development of a coherent mathematical theory of differential equations with discontinuous right-hand side has been, in some sense, pushed forward by its many applications including, for example, numerous problems in automatic control, mechanics and electrical 
engineering, where the prevalent use of switches, relays or digital inputs, is not only customary but, in some cases, also necessary to reach optimal results (see for example Bressan and Piccoli [11], Brogliato [12], Clarke [19]).

The aim of this work is to apply tools of non-autonomous dynamical systems to Carathéodory differential equations, and, in particular, to successfully define a continuous skew-product flow in order to study the qualitative behavior of the solutions. The idea of skew-product flow dates back to the groundbreaking work of Bebutov [7], and it has become a fundamental tool in the study of nonautonomous ordinary differential equations. In order to retrieve a group structure on the evolution of a non-autonomous system (immediate for autonomous systems, where one can build a flow using the solutions), the idea is to jointly track the solution and the evolution of the vector field in time. If these two components are continuous with respect to time, initial vector field and initial data, then the obtained map defines a continuous skew-product flow.

The study of the topologies of continuity for a skew-product flow generated by a Carathéodory differential equations is a classical question which was initially posed by Miller and Sell [41, 42], and then treated by Artstein [3, 4, 5], Heunis [30], Neustadt [43], Opial [45], Sell [52, 53], among many others. Since then, strong and weak $L_{l o c}^{p}$ topologies have been employed to investigate nonautonomous linear differential equations (see Bodin and Sacker [10], Chow and Leiva [20] and Siegmund [55] among others) but, despite its potential interest, the classic theory has not been conveniently developed in the field of non-linear differential equations.

We start this work by harmonizing most of the results contained in Longo et al. [38, 39], and building on top of them, in order to fill some gaps in the original theory, improve its applicability, and allow a more exhaustive analysis of the qualitative behavior of the solutions. In particular, we define new metric topologies and new locally convex vector spaces where the flow map defined by the time-translation is proved to be continuous, and deduce theorems of continuous dependence with respect to the variation of initial data for the solutions of differential problems whose vector fields belong to such spaces. Hence, we obtain the continuity of the skew-product flow composed of the base flow of time translations on the hull of a vector field, and by the solutions of the respective differential problem.

A key ingredient to achieve such results is the thorough study of the so-called $m$-bounds and $l$-bounds of a Carathéodory function $f$, i.e. the families of positive locally integrable functions which respectively serve as a bound for the modulus of $f$ and a Lipschitz coefficient for $f$ on the compact subsets of $\mathbb{R}^{N}$. In fact, the analysis of the $m$-bounds and $l$-bounds allows us to obtain additional topological information. For example, we provide the requisites for the relative compactness of subsets of Lipschitz Carathéodory functions and clarify the conditions (rather weak indeed) under which the (strong or weak) topologies considered in our work, 
and previously in the literature, coincide. Such results are particularly important, not only because they make the whole theory more homogeneous, but also because, wherever applicable, they improve and/or simplify the usable mathematical tools. Indeed, in the first case we obtain, for example, the existence of an invariant ergodic measure for the base flow and thus the possibility of applying results from ergodic theory, whereas in the second case we are allowed to handle the easiest among all the possible topologies, that is, the ones which involve the pointwise convergence. Interestingly, most of the known applications in science and engineering include assumptions which are stronger than the ones for which such results hold.

As a further evidence of the importance of the results of continuity for the skew-product flow, we include a range of theoretical applications. We are able to define two types of linearized skew-product flows, and to obtain the differentiability of the solutions with respect to initial data also for some systems which are not continuously differentiable in the variable $x$, and thus do not admit a classic variational equation. Additionally, we propagate the exponential dichotomy of a system, as well as the structure of the corresponding Sacker-Sell spectrum, over the trajectories of such linearized flows. We also include a result of existence of solutions for specific Carathéodory differential equations whose vector field is possibly discontinuous in the variable $x$ and, as such, is not covered by Carathéodory's theorem. The used approach is completely independent of Filippov theory and relies on Carathéodory's theory and continuity of the solutions with respect to the variation of the vector field.

Furthermore, as a consequence of the previous results, a range of dynamical scenarios is opened in which it is possible to combine techniques of continuous skew-product flows, processes and random dynamical systems (see Arnold [2], Aulbach and Wanner [6], Berger and Siegmund [9], Caraballo and Han [13], Carvalho et al. [16], Johnson et al. [34], Kloeden and Rasmussen [36], Sell [53], Shen and Yi [54] and the references therein). Particularly, by thoroughly calibrating the contemporary use of processes and skew-product formalisms, we provide conditions under which the existence of particular bounded absorbing sets for the process defined by a suitable Carathéodory vector field $f$, allows to deduce the existence of bounded pullback attractors for the processes with vector field belonging to either the alpha-limit set, the omega-limit set, or the whole hull of $f$. Under appropriate assumptions, these theorems also provide the existence of a pullback or a global attractor for the induced skew-product semiflow.

As a final theoretical contribution, we aim to set the path for a generalization of the obtained results to Carathéodory delay differential equations of the type

$$
\dot{x}=f(t, x(t), x(t-\tau)) .
$$

Such a problem has the additional intrinsic difficulty of being infinite dimensional due to the fact that the phase space is $C\left([-\tau, 0], \mathbb{R}^{N}\right)$, i.e. the space of 
continuous functions mapping $[-\tau, 0]$ to $\mathbb{R}^{N}$. Nevertheless, the specific type of delay differential equation in (0.3), together with the fact that $f$ is defined on a finite dimensional space, allow to use some of the already cited techniques and arguments to extend, to some degree and with the unavoidable discrepancies, the theory developed for Carathéodory ODEs to this class of problems. Particularly, we show how to construct a continuous skew-product semiflow from (0.3), again, with respect to several (strong and weak) metric topologies. It is interesting to point out that, despite being a very peculiar class of delay differential problems, equations like (0.3) are widely and successfully used in engineering and applied sciences to modelize many phenomena in which the past affects the future.

Throughout the work, we provide three motivational examples taken from actual models in mechanics (K. Popp and P. Stelter [47]), control theory (Fabbri et al. [25]) and mathematical biology (Rasmussen et al. [50]), and apply some of the obtained results to them. The exposition on such examples is not intended to be exhaustive but to show the wide range of applicability of the results contained in this work.

The theory of Carathéodory differential equations embraces almost one century of mathematical results. From a dynamical systems point of view, it also includes studies of stability, numerical analysis and bifurcation theory (see for example Filippov [26], Osinenko et al [46], Pötzsche and Rasmussen [49], Pötszche [48]). We believe that the topological framework and results contained in this work could be applied also in these directions.

The thesis is structured as follows. In Chapter 1 we set the notation and introduce basic notions and results which serve as preliminary content for the rest of the work. Particularly, the chapter is divided in two sections respectively dealing with Carathéodory ordinary differential equations and the formalism employed in the study of non-autonomous dynamical systems together with a brief summary on the exponential dichotomy and on the dichotomy spectrum. We also include a short description of a simple modelization of a violin string through a Carathéodory differential system.

Chapter 2 is devoted to the topological framework on which is based most of the rest of the following chapters. First, we introduce the spaces of Carathéodory functions. Together with the classical spaces of Lipschitz Carathéodory $(\mathfrak{L} \mathfrak{C})$ and Strong Carathéodory ( $\mathfrak{S} \mathfrak{C}$ ) functions, we consider two new classes of spaces accounting for Carathéodory functions which are possibly discontinuous in the variable $x$, namely, the $\Theta$-Carathéodory $(\Theta \mathfrak{C})$ and weak $\Theta$-Carathéodory $(\mathfrak{W} \Theta \mathfrak{C}$ ) functions. The symbol $\Theta$ stands for a set of moduli of continuity which identify a numerable quantity of compact sets of continuous functions on which the elements in $\Theta \mathfrak{C}$, or respectively $\mathfrak{W} \Theta \mathfrak{C}$, are asked to behave "nicely", that is, somehow continuously in $L_{l o c}^{1}$. The problem of identification of functions in such spaces is also discussed. 
Afterwards, we will define several strong and weak metric topologies of integral type with whom we endow each one of the previously introduced spaces. Among such topologies, a key role will be played by the two new classes of topologies $\mathcal{T}_{\Theta}$ and $\sigma_{\Theta}$. In particular, the sequences in $\left(\Theta \mathfrak{C}, \mathcal{T}_{\Theta}\right)$ and $\left(\mathfrak{W} \Theta \mathfrak{C}, \sigma_{\Theta}\right)$ will be asked to converge (strongly or weakly, respectively) uniformly on the compact sets of continuous functions determined by $\Theta$.

Hence, we focus on the so-called $m$-bounds and $l$-bounds of a Carathéodory function or set of functions. In particular, after introducing the notions of $L_{l o c^{-}}^{p}$ boundedness and $L_{l o c}^{1}$-equicontinuity and relating them to Carathéodory functions through their $m$-bounds and/or $l$-bounds, we show that such properties are inherited by limit functions through the considered topologies and deduce topological results on three important classes of problems: the equivalence of the topologies on suitable subsets, the compactness in $\mathfrak{L} \mathfrak{C}$ and the continuity of the time-translations in $\Theta \mathfrak{C}$ and $\mathfrak{W} \Theta \mathfrak{C}$.

In Chapter 3, we address the core problem of defining a continuous skewproduct flow starting from a Carathéodory ordinary differential equations of the type

$$
\dot{x}=f(t, x), \quad x(0)=x_{0},
$$

and from Carathéodory systems of triangular type as

$$
\begin{cases}\dot{x}=f(t, x), & x(0)=x_{0}, \\ \dot{y}=F(t, x) y+h(t, x), & y(0)=y_{0}\end{cases}
$$

where the function $f(\cdot, \cdot) \in \mathfrak{L} \mathfrak{C}$ admits either $L_{l o c}^{1}$-equicontinuous $m$-bounds or $L_{l o c}^{p}$-bounded $l$-bounds, and $F(\cdot, \cdot)$ and $h(\cdot, \cdot)$ are taken in either $\mathfrak{W} \Theta \mathfrak{C}$ or $\Theta \mathfrak{C}$, so that the problem is well-posed. In particular, notice that $\Theta$ will be determined either thanks to the assumption of $L_{l o c}^{1}$-equicontinuity for the $m$-bounds of $f$ or by the solutions of $\dot{x}=f(t, x)$ when $f$ is in a compact subset of $\mathfrak{L} \mathfrak{C}$.

The triangular system in (0.4) assumes particular relevance if $f$ has continuous partial derivatives with respect to $x, F=J_{x} f \in \mathfrak{S C}$, i.e. $F$ is the Jacobian of $f$ with respect to $x$ and it is a strong Carathéodory function, and $h=0$, that is, the second equation in (0.4) is the variational equation of the first one. Under the assumptions of the theorems for the continuity of the induced skew-product flow, we prove the existence of a linearized skew-product flow (with respect to the topology $\mathcal{T}_{\Theta}$ ) and a $\sigma$-linearized skew-product flow (with respect to the topology $\left.\sigma_{\Theta}\right)$, both composed of the base flow on the hull of $\left(f, J_{x} f\right)$ (with respect to either $\mathcal{T}_{\Theta}$ or $\sigma_{\Theta}$ ) and of the solutions of the respective differential equations. In particular, we show that the solutions of Carathéodory differential equations are differentiable with respect to initial data even in some cases in which the vector field (in the hull of $f$ ) does not have continuous partial derivative with respect to $x$. 
Moreover, we look into the exponential dichotomy and the dichotomy spectrum of the linear system in such linearized skew-product flows and how it propagates thanks to the continuous skew-product flow.

The chapter ends with a section of applications. We start with a theoretical result; a theorem of existence of solutions for differential problems whose vector fields are in $\mathfrak{W} \Theta \mathfrak{C}$, i.e. not necessarily continuous in the space variable. The underlying condition is that such vector fields are limit, in the topology $\sigma_{\Theta}$, of sequences in $\mathfrak{S} \mathfrak{C}$ with $L_{l o c}^{1}$-equicontinuous $m$-bounds. Secondly, we study the impact of the developed theory on a problem of digitization for non-autonomous control systems, and on a non-autonomous and non-linear Carathéodory compartmental system.

Chapter 4 deals with pullback and global attractors for Carathéodory ODEs (whose solutions are defined up to $+\infty$ ) and for the relative continuous skewproduct flow generated thanks to the results in the previous chapter. After providing all the basic definitions and results, we proceed to show how a continuous skew-product flow can be used to infer the existence of an attractor for a set of limit systems. In particular, starting from specific properties on the solutions of an initial problem $\dot{x}=f(t, x)$, we show how it is possible to obtain the existence of a bounded pullback attractor for the processes induced by systems whose vector field is either in the alpha limit set of $f$, in the omega limit set of $f$, or in the whole hull of $f$. Additionally, we give a result of existence of pullback and global attractors for the whole skew-product flow.

Furthermore, we provide sufficient conditions under which the previous results can be applied. In fact, several types of attractors, both for the induced process and the induced skew-product flow, are obtained through comparison results in which the size of the solutions of a Carathéodory differential system $\dot{x}=$ $f(t, x)$ is firstly compared to the size of the solutions of a scalar Carathéodory linear equation and then also to the size of the solutions of a system of linear Carathéodory equations.

Chapter 5 aims to highlight how the theory developed for Carathéodory ODEs can be extended to Carathéodory delay differential equations with constant delay like (0.3). After providing basic preliminaries on Carathéodory delay differential equations with constant delay, we introduce new strong and weak topologies which exhibit a hybrid behavior with respect to the ones presented in Chapter 2. The term hybrid means that, while still asking for a convergence of $L_{l o c}^{1}$ type, we will possibly treat the first $N$ components of the spatial variable (representing the current state in a delay differential equation) in a different way from the last $N$ ones (representing the history of the state). Furthermore, we show how such topologies relate to the ones in Chapter 2 and how to apply or develop, in this new context, some of the previously obtained topological results.

Finally, we prove the continuity of the skew-product semiflow induced by problems of the type $(0.3)$ when $\mathfrak{L} \mathfrak{C}$ is endowed with either two topologies from 
the first part of the work, or the new hybrid topologies (and suitable hypothesis are assumed on $f$ ). As a result, we leave everything ready to develop, wherever it is possible, results analogous to the ones contained in Chapters 3 and 4, but for Carathéodory delay differential equations with constant delay. 



\section{Chapter 1}

\section{Preliminaries}

In this chapter we provide basic definitions and results about Carathéodory differential equations and non-autonomous dynamics. In fact, although the work contains several different topics, as follows we will only include those results and definitions which apply to the whole dissertation or that would break the reading flow if included in the text later. As regards the rest of preliminary notions, we will keep them as close as possible to (or anyway recall them next to) the actual point in which they are used.

Let us start by setting some notation. In the following, we will denote by $\mathbb{R}^{N}$ the $N$-dimensional euclidean space with norm $|\cdot|$, by meas $\mathbb{R}^{N}$ the Lebesgue measure on $\mathbb{R}^{N}$, and by $B_{r}$ the closed ball of $\mathbb{R}^{N}$ centered at the origin and with radius $r$. Notice also that, in some cases in which there is no possibility of misunderstanding, the symbols 0 and 1 will represent the $N$-dimensional vectors composed of zeros and ones, respectively. When $N=1$, we will simply write $\mathbb{R}$ and the symbol $\mathbb{R}^{+}$will denote the set of positive real numbers. Moreover, for any interval $I \subseteq \mathbb{R}$ and any $W \subseteq \mathbb{R}^{N}$, we will use the following notation

$C(I, W)$ : space of continuous functions from $I$ to $W$ endowed with the norm $\|\cdot\|_{\infty}$.

$C_{C}(\mathbb{R})$ : space of real-valued continuous functions with compact support in $\mathbb{R}$, endowed with the norm $\|\cdot\|_{\infty}$. When we want to restrict to the positive continuous functions with compact support in $\mathbb{R}$, we will write $C_{C}^{+}(\mathbb{R})$.

$L^{p}\left(I, \mathbb{R}^{N}\right), 1 \leq p<\infty$ : space of measurable functions from $I$ to $\mathbb{R}^{N}$ whose norm is in the Lebesgue space $L^{p}(I)$.

$L_{l o c}^{p}\left(\mathbb{R}^{N}\right), 1 \leq p<\infty$ : the space of all functions $x(\cdot)$ of $\mathbb{R}$ into $\mathbb{R}^{N}$ such that for every compact interval $I \subset \mathbb{R}, x(\cdot)$ belongs to $L^{p}\left(I, \mathbb{R}^{N}\right)$. When $N=1$, we will simply write $L_{l o c}^{p}$.

The chapter is structured as follows. In Section 1.1 we present classic definitions and results on Carathéodory ordinary differential equations together with a 
motivational example of stick-slip vibration through a Carathéodory modelization of a violin string.

On the other hand, Section 1.2 contains basic notions of non-autonomous dynamics as, for example, the definitions of process, skew-product flow and hull of a function, and of exponential dichotomy and dichotomy spectrum.

\subsection{Carathéodory differential equations}

In this section, we outline the classic results for initial value problems of the type

$$
\dot{x}=f(t, x), \quad x(0)=x_{0},
$$

where $x$ is in $\mathbb{R}^{N}$, and the dependence on $t$ in the right-hand side is possibly just measurable. Particularly, after giving a proper definition of solution for a Cauchy Problem of Carathéodory type, we also provide the statements for the theorems of existence and uniqueness of the solution and continuous variation with respect to initial data. Since such results are classic, their proof are not included in this work and can be found in many textbooks as, for example, Coddington and Levinson [21].

A notion of solution for (1.1) is needed.

Definition 1.1 (Solution for a Carathéodory problem). An absolutely continuous function $x(\cdot): I \subset \mathbb{R} \rightarrow \mathbb{R}^{N}$ such that

- $0 \in I$ and $x(0)=x_{0}$, and

- $\frac{d}{d t} x(t)=f(t, x(t))$ for a.e. $t \in I$

is said to be a solution (in an extended sense) for (1.1).

If $x(\cdot): I \subset \mathbb{R} \rightarrow \mathbb{R}^{N}$ is a solution for (1.1), one can also write the equivalent integral problem

$$
x(t)=x_{0}+\int_{0}^{t} f(s, x(s)) d s .
$$

Carathéodory proved that such an integral problem admits a solution if the righthand side satisfies the conditions which have been named after him.

A function $f: \mathbb{R} \times \mathbb{R}^{N} \rightarrow \mathbb{R}^{M}$ satisfies Carathéodory conditions if

- $f$ is Borel measurable,

- for every compact set $K \subset \mathbb{R}^{N}$ there is a real-valued function $m^{K}(\cdot) \in L_{l o c}^{1}$ such that for almost every $t \in \mathbb{R}$, one has

$$
|f(t, x)| \leq m^{K}(t) \quad \text { for all } x \in K \text {. }
$$


- for almost every $t \in \mathbb{R}, f(t, \cdot)$ is continuous.

Later on, we will refer to the function $m(\cdot)$ as an $m$-bound for $f$ on $K$.

As follows, we state a theorem of existence and uniqueness of the solution for a Cauchy Problem of Carathéodory type. As one may expect, some kind of Lipschitz continuity in the variable $x$ is necessary. We will refer to the function $l(\cdot)$, which acts as a Lipschitz coefficient for $f$, as an $l$-bound for $f$. The notation, used within this statement will be used in the rest of the work.

Theorem 1.2. Let $f: \mathbb{R} \times \mathbb{R}^{N} \rightarrow \mathbb{R}^{N}$ satisfy Carathéodory conditions and also be locally Lipschitz continuous, i.e. for every compact set $K \subset \mathbb{R}^{N}$ there exists a real-valued function $l^{K}(\cdot) \in L_{\text {loc }}^{1}$ such that for almost every $t \in \mathbb{R}$ one has

$$
|f(t, x)-f(t, y)| \leq l^{K}(t)|x-y| \quad \text { for all } x, y \in K .
$$

Then, for any $x_{0} \in \mathbb{R}^{N}$ there exists a maximal interval $I_{f, x_{0}}=\left(a_{f, x_{0}}, b_{f, x_{0}}\right)$ and a unique continuous function $x\left(\cdot, f, x_{0}\right)$ defined on $I_{f, x_{0}}$ which is the solution of the Cauchy Problem

$$
\dot{x}=f(t, x), \quad x(0)=x_{0} .
$$

In particular, if $a_{f, x_{0}}>-\infty$ (resp. $\left.b_{f, x_{0}}<\infty\right)$, then $\left|x\left(t, f, x_{0}\right)\right| \rightarrow \infty$ as $t \downarrow a_{f, x_{0}}$ (resp. as $\left.t \uparrow b_{f, x_{0}}\right)$.

A proof of 1.2 can be found in [21, Theorem 1.1, p.43, Theorem 1.2, p.45 and Theorem 2.2, p.49].

Moreover, a theorem of continuous dependence of the solutions with respect to the initial conditions holds true, too (see [21, Theorems 4.2 and 4.3, p.59]).

Theorem 1.3. Let $f: \mathbb{R} \times \mathbb{R}^{N} \rightarrow \mathbb{R}^{N}$ satisfy Carathéodory conditions and also be locally Lipschitz continuous in $x$ for each fixed $t \in \mathbb{R}$. Then, there exists a $\delta>0$ such that for any $(\tau, \xi) \in \mathbb{R} \times \mathbb{R}^{N}$, if

$$
\left|\tau-t_{0}\right|+\left|\xi-x_{0}\right|<\delta
$$

then all the solutions $x(\cdot, \tau, \xi)$ of

$$
\dot{x}=f(t, x), \quad x(\tau)=\xi .
$$

exist over some interval $[a, b] \subset I_{f, t_{0}, x_{0}}$. Moreover, as $(\tau, \xi) \rightarrow\left(t_{0}, x_{0}\right)$, one has that

$$
x(\cdot, \tau, \xi) \rightarrow x\left(\cdot, t_{0}, x_{0}\right),
$$

uniformly on $[a, b]$.

Example 1.4. Stick-slip vibration is a phenomenon occurring in physics and reality when two bodies $\mathrm{A}$ and $\mathrm{B}$ are in contact through a surface and one of them, namely $\mathrm{A}$, is subject to an elastic constraint whereas the other, call it B, 


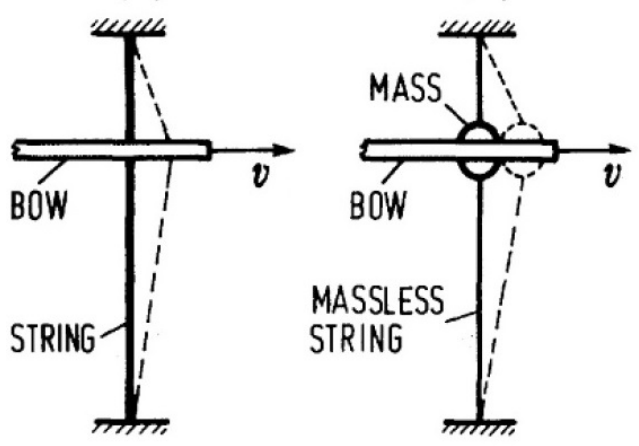

(a)

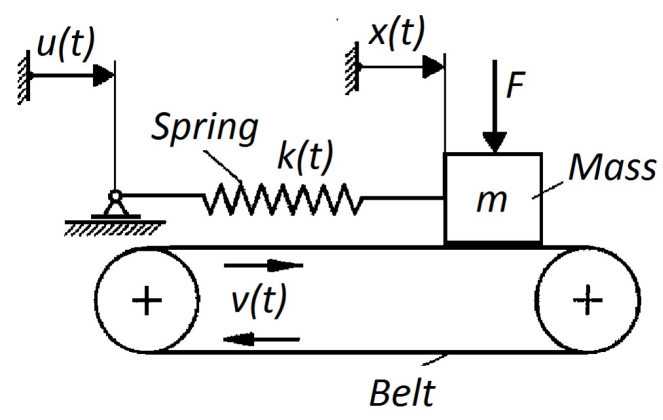

(b)

Figure 1.1: Examples of stick-slip vibration in a bow-rubbed string (a) and in a mechanical model (b).

can be moved in a direction which is parallel to such contact surface. Due to static friction, when the body B starts to move, the body A will move integrally till the elastic force will balance the friction and the body A will begin to slide back to its original position. At this point the process reiterates.

An interesting example of this phenomenon is given by bowed string instruments where the sound is produced when the strings are rubbed by a bow (see Figure 1.1a). In this case, A would be represented by one of the strings, while B is the bow.

An equivalent mechanical example is portrayed in Figure 1.1b, where a body is connected to a wall through a spring and it is positioned on top of a belt that can move longitudinally. For more information, see [47]. The system is governed by the following equation

$$
m \ddot{x}=F_{k}+F_{f}
$$

where $F_{k}$ represents the force due to the spring and $F_{f}$ the one due to the friction. In full generality such forces can depend explicitly on time and/or on the state in a possibly nonlinear way. That is

$$
F_{k}=-k(t, x) x \quad \text { and } \quad\left|F_{f}\right| \leq \mu(t, \dot{x})|F|,
$$

where $k(\cdot, \cdot)$ represents the coefficient of elasticity of the spring, $\mu(\cdot, \cdot)$ the coefficient of static friction and $F$ the gravitational force.

In fact, in this work we are interested in those cases in which the functions $k(\cdot, \cdot)$ and $\mu(\cdot, \cdot)$ are not necessarily continuous on time. Just to go back to the example of bowed string instruments, the elasticity of the string can be abruptly changed by the player anytime he suddenly presses it with his fingers to play a different note. If $k(\cdot, \cdot)$ and $\mu(\cdot, \cdot)$ satisfies the Carathéodory conditions, this problem can be treated within the framework of this work. 


\subsection{Basic notions of non-autonomous dynamics}

\subsubsection{Processes and skew product flows}

When dealing with autonomous differential equations, it is well known that the behavior of the solutions depends on the elapsed time more than the initial and the final time. However, in non-autonomous dynamics, both the initial and the final time are crucial to understand the behavior of the solutions of a dynamical system. As follows, we introduce the two formalisms used in non-autonomous dynamics that allow to take into account this double dependence on time and that will be used in the rest of the work.

Definition 1.5 (Process). A process on a metric space $(X, d)$ is a family of continuous maps $\{S(t, s) \mid t \geq s\} \subset \mathcal{C}(X)$ satisfying

- $S(t, t) x=x$ for every $t \in \mathbb{R}$ and $x \in X$.

- $S(t, s)=S(t, r) S(r, s)$, for every $t \geq r \geq s$.

- $(t, s, x) \mapsto S(t, s) x$ is continuous for every $t \geq s$ and $x \in X$.

A process can be induced by a non-autonomous differential equation if one assumes that, for any $r \in \mathbb{R}$ and any $x_{0} \in \mathbb{R}^{N}$, the initial value problem $\dot{x}=$ $f(t, x), x(r)=x_{0}$ has a unique solution $x\left(\cdot, f, r, x_{0}\right)$ defined on $[r, \infty)$. In fact, one can pose

$$
S_{f}(t+r, r) x_{0}=x\left(t+r, f, r, x_{0}\right),
$$

where $t \geq 0$ and $r \in \mathbb{R}$ and easily check that the properties in Definition 1.5 are satisfied.

Before giving the definition of skew-product flow, let us clarify what one means by a continuous flow.

Definition 1.6 (Continuous flow). Consider a metric space $(E, d)$. A continuous flow on $E$ is a continuous group action of the additive group of real numbers on $E$, that is, a continuous map $\theta: \mathbb{R} \times E \rightarrow E$ such that for all $\omega \in E$ and all $s, t \in \mathbb{R}$ one has

$$
\theta(0, \omega)=\omega \quad \text { and } \quad \theta(t+s, \omega)=\theta(t, \theta(s, \omega)) .
$$

Definition 1.7 (Skew-product flow). Let $\left(X, d_{1}\right)$ and $\left(E, d_{2}\right)$ be metric spaces, and consider two continuous applications $\theta: \mathbb{R} \times E \rightarrow E$ and $\varphi: \mathbb{R} \times E \times X \rightarrow X$. The pair $(\theta, \varphi): \mathbb{R} \times E \times X \rightarrow E \times X$ is called a continuous skew-product flow if

- $\theta$ is a continuous flow.

- For any $\omega \in E$ and $x \in X$ one has

$$
\varphi(0, \omega, x)=x \quad \text { (Initial value condition) }
$$


- For any $\omega \in E, x \in X$ and $t, s \in \mathbb{R}$ one has

$$
\varphi(t+s, \omega, x)=\varphi(t, \theta(s, \omega), \varphi(s, \omega, x)) \quad \text { (Cocycle property) } .
$$

Particularly, if $X$ is a Banach space and $\varphi$ is linear in $x$, then $(\theta, \varphi)$ is called a linear skew-product flow. Moreover, if $\varphi$ is defined only on $\mathbb{R}^{+} \times E \times X$, then $(\theta, \varphi)$ is called a continuous skew-product semiflow. Additionally, if $\phi$ is defined on a subset $\mathcal{U}$ of $\mathbb{R} \times E \times X$ (resp. $\left.\mathbb{R}^{+} \times E \times X\right)$, then $(\theta, \varphi)$ is called a local continuous skew-product flow (resp. semiflow).

Remark 1.8. A skew-product flow is in fact a flow on $E \times X$ as stated in Definition 1.6. The term skew-product is referred to the fact that it is composed of two components so that the first affects the second one but the opposite is not true.

Before seeing how a non-autonomous differential equation can induce a (local) continuous skew product flow (or semiflow) we introduce the notion of timetranslation and hull of a function.

Definition 1.9 (Time-translation). Consider a Banach space $X$ and a function $f: \mathbb{R} \times X \rightarrow X$. We call time-translation at time $t$ of $f$, the function

$$
f_{t}: \mathbb{R} \times X \rightarrow X, \quad(s, x) \mapsto f_{t}(s, x)=f(s+t, x) .
$$

Definition 1.10 (Hull of a function). Let $(E, d)$ be a metric space of functions mapping $\mathbb{R} \times X$ onto $X$, where $X$ is a metric space, and let $\mathcal{T}$ be the topology induced by the metric. If $f \in E$, and for any $t \in \mathbb{R}$ also $f_{t} \in E$, where $f_{t}$ is the time translation at time $t$ of $f$ (see Definition 1.9), then we call the hull of $f$ with respect to $(E, \mathcal{T})$, the metric subspace of $(E, \mathcal{T})$ defined by

$$
\operatorname{Hull}_{(E, \mathcal{T})}(f)=\left(\operatorname{cls}_{(E, \mathcal{T})}\left\{f_{t} \mid t \in \mathbb{R}\right\}, \mathcal{T}\right)
$$

where, $\operatorname{cls}_{(E, \mathcal{T})}(A)$ represents the closure in $(E, \mathcal{T})$ of the set $A$ and $\mathcal{T}$ is the induced topology.

Consider a differential equation $\dot{x}=f(t, x)$, where $f$ belongs to a suitable metric space $(E, d)$ of functions mapping $\mathbb{R} \times X$ to $X$, and let $\mathcal{T}$ be the topology induced by the metric. For any $x_{0} \in X$ and $g \in \operatorname{Hull}_{(E, \mathcal{T})}(f)$, let $x\left(\cdot, g, x_{0}\right)$ be the unique solution at time $t$ of the initial value problem $\dot{x}=g(t, x), x(0)=x_{0}$ and let $I_{g, x_{0}}$ be its maximal interval of definition. Define

$$
\mathcal{U}=\bigcup_{\substack{g \in \operatorname{Hull}_{(E, \mathcal{T})}(f), x \in X}}\left\{(t, g, x) \mid t \in I_{g, x}\right\}
$$

If the maps

$$
\theta: \mathbb{R} \times \operatorname{Hull}_{(E, \mathcal{T})}(f) \rightarrow \operatorname{Hull}_{(E, \mathcal{T})}(f),(t, g) \mapsto g_{t},
$$


and

$$
\varphi: \mathcal{U} \subset \mathbb{R} \times \operatorname{Hull}_{(E, \mathcal{T})}(f) \times X \rightarrow X,\left(t, g, x_{0}\right) \mapsto x\left(t, g, x_{0}\right)
$$

are continuous, then $(\theta, \varphi)$ is a local continuous skew-product flow induced by the differential equation $\dot{x}=f(t, x)$. Notice in particular that for a linear differential problem one obtains a continuous linear skew-product flow.

Remark 1.11. Notice that the problem of giving sufficient conditions for the continuity of $\theta$ and $\varphi$ in the case of a Carathéodory differential equation is one of the core problems of this work and the actual content of most of Chapter 3.

Example 1.12. As follows, we aim to present a classic and well-studied case of skew-product flow induced by a non-autonomous ordinary differential equation. Specifically, consider

$$
\dot{x}=f(t, x),
$$

with $f \in C\left(\mathbb{R} \times \mathbb{R}^{N}, \mathbb{R}^{N}\right)$ and such that for any compact set $K \subset \mathbb{R}^{N}$ there is a constant $l^{K}>0$ for which the following inequality holds

$$
\left|f\left(t, x_{1}\right)-f\left(t, x_{2}\right)\right| \leq l^{K}\left|x_{1}-x_{2}\right| \quad \text { for all } x_{1}, x_{2} \in K \text { and } t \in \mathbb{R} .
$$

Let us endow the space $C\left(\mathbb{R} \times \mathbb{R}^{N}, \mathbb{R}^{N}\right)$ with the compact-open topology $\mathcal{T}_{\text {co }}$, i.e. the topology of uniform convergence on the compact subsets of $\mathbb{R} \times \mathbb{R}^{N}$. It is well-known that $\left(C\left(\mathbb{R} \times \mathbb{R}^{N}, \mathbb{R}^{N}\right), \mathcal{T}_{c o}\right)$ is a complete metric space and it is easy to prove that the application

$$
\theta: \mathbb{R} \times C\left(\mathbb{R} \times \mathbb{R}^{N}, \mathbb{R}^{N}\right) \rightarrow C\left(\mathbb{R} \times \mathbb{R}^{N}, \mathbb{R}^{N}\right), \quad(t, g) \mapsto g_{t},
$$

is a continuous flow. Moreover, it is also straightforward to see that the hull of $f$ in $\left(C\left(\mathbb{R} \times \mathbb{R}^{N}, \mathbb{R}^{N}\right), \mathcal{T}_{\text {co }}\right)$, as constructed in Definition 1.10, satisfies

$$
\operatorname{Hull}_{\left(C\left(\mathbb{R} \times \mathbb{R}^{N}, \mathbb{R}^{N}\right), \mathcal{T}_{c o}\right)}(f) \subset C\left(\mathbb{R} \times \mathbb{R}^{N}, \mathbb{R}^{N}\right),
$$

and that (1.2) also holds for any function $g \in \operatorname{Hull}_{\left(C\left(\mathbb{R} \times \mathbb{R}^{N}, \mathbb{R}^{N}\right), \mathcal{T}_{c o}\right)}(f)$. As a consequence, for every $\left(t_{0}, x_{0}\right) \in \mathbb{R} \times \mathbb{R}^{N}$ there exists precisely one solution for

$$
\dot{x}=g(t, x), \quad g \in \operatorname{Hull}_{\left(C\left(\mathbb{R} \times \mathbb{R}^{N}, \mathbb{R}^{N}\right), \mathcal{T}_{c o}\right)}(f) .
$$

By simplicity, assume that all the solutions of any problem like (1.4) are defined on the whole real line. Thanks to [53, Theorem IV.3, p.62], one has that the map

$$
\begin{array}{ccc}
\Pi: \mathbb{R} \times \operatorname{Hull}_{\left(C\left(\mathbb{R} \times \mathbb{R}^{N}, \mathbb{R}^{N}\right), \mathcal{T}_{\text {co }}\right)}(f) \times \mathbb{R}^{N} & \rightarrow \operatorname{Hull}_{\left(C\left(\mathbb{R} \times \mathbb{R}^{N}, \mathbb{R}^{N}\right), \mathcal{T}_{\text {co }}\right)}(f) \times \mathbb{R}^{N} \\
\left(t, g, x_{0}\right) & \mapsto & \left(g_{t}, x\left(t, g, x_{0}\right)\right)
\end{array}
$$

defines a continuous skew-product flow on $\mathbb{R} \times \operatorname{Hull}_{\left(C\left(\mathbb{R} \times \mathbb{R}^{N}, \mathbb{R}^{N}\right), \mathcal{T}_{\text {co }}\right)}(f)$. Additionally the following result holds true.

Theorem 1.13. Consider $f \in C\left(\mathbb{R} \times \mathbb{R}^{N}, \mathbb{R}^{N}\right)$. The following statements are equivalent.

(i) $f$ is uniformly continuous and bounded in $\mathbb{R} \times K$ for every compact set $\mathcal{K} \subset \mathbb{R}^{N}$

(ii) $\operatorname{Hull}_{\left(C\left(\mathbb{R} \times \mathbb{R}^{N}, \mathbb{R}^{N}\right), \mathcal{T}_{c o}\right)}(f)$ is compact. 


\subsubsection{Exponential dichotomy and dichotomy spectrum}

Consider a linear continuous skew-product flow on $E \times \mathbb{R}^{N}$, where $E$ is a metric space, i.e.

$$
\begin{array}{clc}
\Psi: \mathbb{R} \times E \times \mathbb{R}^{N} & \rightarrow & E \times \mathbb{R}^{N} \\
\left(t, \omega, y_{0}\right) & \mapsto \quad\left(\theta(t, \omega), \Phi(t, \omega) y_{0}\right),
\end{array}
$$

where $\Phi(t, \omega)$ denotes the fundamental matrix solution of the linear differential problem

$$
\dot{y}=A(\theta(t, \omega)) y
$$

with $A(\theta(\cdot, \omega)) \in L_{l o c}^{1}\left(\mathbb{R}^{N \times N}\right)$ for any $\omega \in E$ so to have existence and uniqueness of the solutions, and $\Phi(0, \omega)=\mathrm{I}_{N}$.

Definition 1.14 (Exponential dichotomy). Let $I$ be one of the half-lines $(-\infty, 0]$, $[0, \infty)$ or the real line $\mathbb{R}$ and let $\Delta$ be a subset of $E$. We say that the linear skewproduct flow (1.6), has exponential dichotomy on $I$ over the set $\Delta$ if there are a continuous family of projections $P: \Delta \rightarrow \mathcal{L}\left(\mathbb{R}^{N}, \mathbb{R}^{N}\right), \omega \mapsto P(\omega)$, and constants $K \geq 1$ and $\alpha>0$, such that for every $s, t \in I$ and every $\omega \in \Delta$

$$
\begin{array}{ll}
\left\|\Phi(t, \omega) P(\omega) \Phi^{-1}(s, \omega)\right\| \leq K e^{-\alpha(t-s)} & \text { if } \quad t \geq s \\
\left\|\Phi(t, \omega)\left(\mathrm{I}_{N}-P(\omega)\right) \Phi^{-1}(s, \omega)\right\| \leq K e^{\alpha(t-s)} & \text { if } \quad t \leq s .
\end{array}
$$

When $\Delta$ reduces to a point $\omega$, we say that the corresponding system $\dot{y}=A\left(\theta_{t} \omega\right) y$, has exponential dichotomy on $I$. If $I=\mathbb{R}$ we will simply say that the linear skewproduct flow (resp. the system) has exponential dichotomy.

We recall the definition of dichotomy spectrum, or Sacker-Sell spectrum for a non-autonomous linear ODE and for a linear skew-product flow.

Definition 1.15 (Dichotomy spectrum and resolvent set over a trajectory). Let $\omega \in E$ be fixed. The dichotomy spectrum of $\dot{y}=A(\theta(t, \omega)) y$, which will be denoted by $\Sigma(\omega)$, is the set of $\gamma \in \mathbb{R}$ such that $\dot{y}=\left[A(\theta(t, \omega))-\gamma \mathrm{I}_{N}\right] y$ does not have exponential dichotomy. The resolvent set is $\rho(\omega)=\mathbb{R} \backslash \Sigma(\omega)$.

Such notion can be generalized to a family of non-autonomous linear systems generated by the elements of a subset of the set $E$.

Definition 1.16 (Dichotomy spectrum for a linear skew-product flow). Let $\Delta$ be a subset of $E$. The dichotomy spectrum of the linear skew-product flow (1.6) over $\Delta$, denoted by $\Sigma(\Delta)$ is the set of $\gamma \in \mathbb{R}$ such that the family $\dot{y}=[A(\theta(t, \omega))-$ $\left.\gamma \mathrm{I}_{N}\right] y$ does not have exponential dichotomy over $\Delta$.

When $\Delta$ is an invariantly connected compact invariant set of $E$, Sacker and Sell [51, Theorem 2 p.334] proved that $\Sigma(\Delta)$ is the union of $0 \leq k \leq N$ compact intervals

$$
\Sigma(\Delta)=\left[a_{1}, b_{1}\right] \cup \cdots \cup\left[a_{k}, b_{k}\right]
$$


where $1 \leq k \leq N$ and $a_{1} \leq b_{1}<a_{2} \leq b_{2}<\cdots \leq a_{k} \leq b_{k}$.

When dealing with invariant sets which are not necessarily compact, Siegmund's approach in [55], for linear skew-product flows induced by measurable linear differential systems, turns out to be very useful to study the dichotomy spectrum $\Sigma(\omega)$ and the respective decomposition of $\mathbb{R} \times \mathbb{R}^{N}$ in spectral manifolds.

In order to properly introduce it, let us give the definition of linear integral manifold and of $\gamma^{+}$and $\gamma^{-}$-quasibounded functions, together with some additional notions and properties.

Definition 1.17 (Linear integral manifold). A nonempty set $\mathcal{W} \subset \mathbb{R} \times \mathbb{R}^{N}$ is a linear integral manifold of (1.7) if

(i) it is invariant, i.e. $(\tau, y) \in \mathcal{W} \Rightarrow\left(t, \Phi(t, \omega) \Phi^{-1}(\tau, \omega) y\right) \in \mathcal{W}$ for all $t \in \mathbb{R}$.

(ii) For every $\tau \in \mathbb{R}$ the fiber $\mathcal{W}(\tau)=\left\{y \in \mathbb{R}^{N} \mid(\tau, y) \in \mathcal{W}\right\}$ is a linear subspace of $\mathbb{R}^{N}$.

Because of the invariance, the fibers of a linear integral manifold have constant dimension. Moreover, it is easy to prove that $\mathbb{R} \times \mathbb{R}^{N}$ and $\mathbb{R} \times\{0\}$ are always linear integral manifolds for any system like (1.7). Notice also that a linear integral manifold is a topological manifold in $\mathbb{R} \times \mathbb{R}^{N}$ and a vector bundle on $\mathbb{R}$. In particular, if $\mathcal{W}_{1}$ and $\mathcal{W}_{2}$ are linear integral manifolds of (1.7), then also the intersection and the sum

$$
\begin{aligned}
& \mathcal{W}_{1} \cap \mathcal{W}_{2}:=\left\{(\tau, y) \in \mathbb{R} \times \mathbb{R}^{N} \mid y \in \mathcal{W}_{1}(\tau) \cap \mathcal{W}_{2}(\tau)\right\} \\
& \mathcal{W}_{1}+\mathcal{W}_{2}:=\left\{(\tau, y) \in \mathbb{R} \times \mathbb{R}^{N} \mid y \in \mathcal{W}_{1}(\tau)+\mathcal{W}_{2}(\tau)\right\}
\end{aligned}
$$

are linear integral manifolds of (1.7). Specifically, a sum $\mathcal{W}_{1}+\cdots+\mathcal{W}_{n}$ of linear integral manifolds is said to be a Whitney-sum $\mathcal{W}_{1} \oplus \cdots \oplus \mathcal{W}_{n}$ if for any $i=1, \ldots, n-1$ one has $\left(\mathcal{W}_{1}+\cdots+\mathcal{W}_{i}\right) \cap \mathcal{W}_{i+1}=\mathbb{R} \times\{0\}$.

On the other hand, we can describe the exponential growth of a continuous function through the following definition.

Definition $1.18\left(\gamma^{+}\right.$-quasibounded and $\gamma^{-}$-quasibounded functions). Consider $\gamma \in \mathbb{R}$. A continuous function $f: \mathbb{R} \rightarrow \mathbb{R}^{N}$ is

- $\gamma^{+}$-quasibounded if $\sup _{t \geq 0}|f(t)| e^{-\gamma t}<\infty$,

- $\gamma^{-}$-quasibounded if $\sup _{t \leq 0}|f(t)| e^{-\gamma t}<\infty$.

In particular, one can prove that for any $\gamma \in \mathbb{R}$, the sets

$$
\begin{aligned}
& \mathcal{S}_{\gamma}:=\left\{(\tau, y) \in \mathbb{R} \times \mathbb{R}^{N} \mid \Phi(\cdot, \omega) \Phi^{-1}(\tau, \omega) y \text { is } \gamma^{+} \text {-quasibounded }\right\}, \\
& \mathcal{U}_{\gamma}:=\left\{(\tau, y) \in \mathbb{R} \times \mathbb{R}^{N} \mid \Phi(\cdot, \omega) \Phi^{-1}(\tau, \omega) y \text { is } \gamma^{-} \text {-quasibounded }\right\}
\end{aligned}
$$

are linear integral manifolds of (1.7). Then, we can finally state the following spectral theorem (see [55, Spectral Theorem p.249] for the proof). 
Theorem 1.19. The dichotomy spectrum $\Sigma(\omega)$ of (1.7) is the disjoint union of $k$ closed intervals (called spectral intervals) where $0 \leq k \leq N$. That is, $\Sigma(\omega)$ is empty, it is the whole $\mathbb{R}$, or there exists $k \in \mathbb{N}$, with $1 \leq k \leq N-1$, such that

$$
\Sigma(\omega)=I_{1} \cup\left[a_{2}, b_{2}\right] \cup \cdots \cup\left[a_{k-1}, b_{k-1}\right] \cup I_{k},
$$

where $I_{1}$ is either $\left[a_{1}, b_{1}\right]$ or $\left(-\infty, b_{1}\right], I_{k}$ is either $\left[a_{k}, b_{k}\right]$ or $\left[a_{k}, \infty\right)$, and $a_{1} \leq$ $b_{1}<a_{2} \leq b_{2}<\cdots \leq a_{k} \leq b_{k}$. Moreover, choose

$$
\gamma_{0} \in \rho(\omega) \quad \text { with }\left(-\infty, \gamma_{0}\right) \subset \rho(\omega) \text { if possible, }
$$

otherwise, define $\mathcal{U}_{\gamma_{0}}:=\mathbb{R} \times \mathbb{R}^{N}$ and $\mathcal{S}_{\gamma_{0}}:=\mathbb{R} \times\{0\} ;$ choose

$$
\gamma_{n} \in \rho(\omega) \quad \text { with }\left(\gamma_{n}, \infty\right) \subset \rho(\omega) \text { if possible, }
$$

otherwise, define $\mathcal{U}_{\gamma_{n}}:=\mathbb{R} \times\{0\}$ and $\mathcal{S}_{\gamma_{n}}:=\mathbb{R} \times \mathbb{R}^{N}$. Then, the sets

$$
\mathcal{W}_{0}=\mathcal{S}_{\gamma_{0}} \quad \text { and } \quad \mathcal{W}_{k}=\mathcal{U}_{\gamma_{k}}
$$

are linear integral manifolds for (1.7). Additionally, for $k \geq 2$ choose any $\gamma_{i} \in$ $\rho(\omega)$ with

$$
b_{i} \leq \gamma_{i} \leq a_{i+1} \quad \text { for } i=1, \ldots, k-1 .
$$

Then, for every $i=1, \ldots, k-1$, the intersection

$$
\mathcal{W}_{i}=\mathcal{U}_{\gamma_{i-1}} \cap \mathcal{S}_{\gamma_{i}}
$$

is a linear integral manifolds for (1.7) with $\operatorname{dim} \mathcal{W}_{i} \geq 1$. The linear integral manifolds $\mathcal{W}_{i}$, with $i=0 \ldots, k$ are called spectral manifolds and they are independent of the choice of $\gamma_{i}$. Furthermore

$$
\mathbb{R} \times \mathbb{R}^{N}=\mathcal{W}_{0} \oplus \cdots \oplus \mathcal{W}_{k+1}
$$

The following result is also important because it gives sufficient condition for which the dichotomy spectrum in Theorem 1.19 reduces to the Sacker-Sell's spectrum (see [55, Theorem 3.1 p.253] for the proof).

Theorem 1.20. The following statements are equivalent

(i) The system $\dot{y}=A(\theta(t, \omega))$ y has bounded growth, i.e. there exist constants $K \geq 1$ and $\alpha \geq 0$ such that

$$
\left\|\Phi(t, \omega) \Phi^{-1}(s, \omega)\right\| \leq K e^{\alpha|t-s|} \quad \text { for } \quad t, s \in \mathbb{R}
$$

(ii) The system $\dot{y}=A(\theta(t, \omega))$ y has a nonempty and compact dichotomy spectrum

$$
\Sigma(\omega)=\left[a_{1}, b_{1}\right] \cup \cdots \cup\left[a_{k}, b_{k}\right] \quad \text { with } 1 \leq k \leq N,
$$

and the spectral manifolds $\mathcal{W}_{0}$ and $\mathcal{W}_{k+1}$ are trivial, i.e.

$$
\mathbb{R} \times \mathbb{R}^{N}=\mathcal{W}_{1} \oplus \cdots \oplus \mathcal{W}_{k} .
$$




\section{Chapter 2}

\section{Spaces and Topologies}

This chapter deals with the introduction of the topological spaces and notions that will be used for most of the rest of the work.

In Section 2.1 we will present all the spaces that will be used for the study of Carathéodory ordinary differential equations. Together with the classical spaces of Lipschitz Carathéodory and Strong Carathéodory functions, we introduce two more spaces accounting for Carathéodory functions which are possibly discontinuous in the variable $x$ and that will play an important role in the rest of the work. The problem of identification of functions differing only on a negligible set is also discussed.

In Section 2.2 we endow the spaces presented in Section 2.1 with suitable metric topologies and we also present a first technical lemma which makes the space $\mathfrak{W} \Theta \mathfrak{C}\left(\mathbb{R}^{M}\right)$ easier to be used.

In Section 2.3 we look deeper into the so-called $m$-bounds and $l$-bounds of a Carathéodory function or set of functions. We introduce the notions of $L_{l o c}^{p}$-boundedness and $L_{l o c}^{1}$-equicontinuity and relate them to a Carathéodory function or set of functions through the $m$-bounds and/or $l$-bounds.

In Sections 2.4 to 2.7 we develop some applications of the $L_{l o c}^{p}$-boundedness and $L_{l o c}^{1}$-equicontinuity for Carathéodory functions that will be useful in the following chapters.

\section{$2.1 \quad$ Spaces}

Consider $1 \leq p<\infty$ and denote by $\mathfrak{C}_{p}\left(\mathbb{R}^{M}\right)$ (or simply $\mathfrak{C}_{p}$ when $M=N$ ), the set of functions $f: \mathbb{R} \times \mathbb{R}^{N} \rightarrow \mathbb{R}^{M}$ satisfying

(C1) $f$ is Borel measurable and

(C2) for every compact set $K \subset \mathbb{R}^{N}$ there exists a real-valued function $m^{K} \in L_{l o c}^{p}$, called $m$-bound in the following, such that for almost every $t \in \mathbb{R}$, one has

$$
|f(t, x)| \leq m^{K}(t) \quad \text { for all } x \in K .
$$


The assumptions (C1) and (C2) are called Carathéodory conditions from the name of the Greek mathematician Constantin Carathéodory who proved the existence of solutions for ordinary differential equations whose vector fields satisfy such conditions together with the continuity in the variable $x$ for almost every $t \in \mathbb{R}$. As follows, we introduce the sets of Carathéodory functions which are used in this work.

Definition 2.1 (Lipschitz Carathéodory functions). A function $f: \mathbb{R} \times \mathbb{R}^{N} \rightarrow$ $\mathbb{R}^{M}$ is said to be Lipschitz Carathéodory for $1 \leq p<\infty$, and we will write $f \in \mathfrak{L} \mathfrak{C}_{p}\left(\mathbb{R}^{M}\right)$ (or simply $f \in \mathfrak{L} \mathfrak{C}_{p}$ when $M=N$ ), if it satisfies (C1), (C2) and

(L) for every compact set $K \subset \mathbb{R}^{N}$ there exists a real-valued function $l^{K} \in L_{l o c}^{p}$ such that for almost every $t \in \mathbb{R}$ one has

$$
|f(t, x)-f(t, y)| \leq l^{K}(t)|x-y| \quad \text { for all } x, y \in K .
$$

In particular, for any compact set $K \subset \mathbb{R}^{N}$, we refer to the optimal $m$-bound and the optimal l-bound of $f$ as to

$$
m^{K}(t)=\sup _{x \in K}|f(t, x)| \quad \text { and } \quad l^{K}(t)=\sup _{\substack{x, y \in K \\ x \neq y}} \frac{|f(t, x)-f(t, y)|}{|x-y|}
$$

respectively. Clearly, for any compact set $K \subset \mathbb{R}^{N}$ the suprema in (2.2) can be taken for a countable dense subset of $K$ leading to the same actual definition, which guarantees that the functions defined in (2.2) are measurable.

Definition 2.2 (Strong Carathéodory functions). A function $f: \mathbb{R} \times \mathbb{R}^{N} \rightarrow \mathbb{R}^{M}$ is said to be strong Carathéodory for $1 \leq p<\infty$, and we will write $f \in \mathfrak{S C}_{p}\left(\mathbb{R}^{M}\right)$ (or simply $f \in \mathfrak{S C}_{p}$ when $M=N$ ), if it satisfies (C1), (C2) and

(S) for almost every $t \in \mathbb{R}$, the function $f(t, \cdot)$ is continuous.

The optimal m-bound for a strong Carathéodory function on any compact set $K \subset \mathbb{R}^{N}$, is defined exactly as in equation (2.2).

Functions which are not necessarily continuous in the second variable are also considered. First, we set some notation.

Definition 2.3 (Suitable set of moduli of continuity). We call a suitable set of moduli of continuity, any countable set of non-decreasing continuous functions

$$
\Theta=\left\{\theta_{j}^{I} \in C\left(\mathbb{R}^{+}, \mathbb{R}^{+}\right) \mid j \in \mathbb{N}, I=\left[q_{1}, q_{2}\right], q_{1}, q_{2} \in \mathbb{Q}\right\}
$$

such that $\theta_{j}^{I}(0)=0$ for every $\theta_{j}^{I} \in \Theta$, and with the relation of partial order given by

$$
\theta_{j_{1}}^{I_{1}} \leq \theta_{j_{2}}^{I_{2}} \text { whenever } I_{1} \subseteq I_{2} \text { and } j_{1} \leq j_{2}
$$


Now we introduce the family of sets $\Theta \mathfrak{C}_{p}\left(\mathbb{R}^{M}\right)$ and $\mathfrak{W} \Theta \mathfrak{C}_{p}\left(\mathbb{R}^{M}\right)$, where $\Theta$ is a suitable set of moduli of continuity.

Definition 2.4 ( $\Theta$-Carathéodory and weak $\Theta$-Carathéodory functions). Let $\Theta$ be a suitable set of moduli of continuity as in Definition 2.3, and $\mathcal{K}_{j}^{I}$ the compact set of functions in $C\left(I, B_{j}\right)$ which admit $\theta_{j}^{I}$ as a modulus of continuity.

- We say that a function $f: \mathbb{R} \times \mathbb{R}^{N} \rightarrow \mathbb{R}^{M}$ is $\Theta$-Carathéodory for $1 \leq p<\infty$ and write $f \in \Theta \mathfrak{C}_{p}\left(\mathbb{R}^{M}\right.$ ) (or simply $f \in \Theta \mathfrak{C}_{p}$ when $M=N$ ), if $f$ satisfies (C1), (C2), and

(T) for each $j \in \mathbb{N}$ and $I=\left[q_{1}, q_{2}\right], q_{1}, q_{2} \in \mathbb{Q}$, if $\left(x_{n}(\cdot)\right)_{n \in \mathbb{N}}$ is a sequence in $\mathcal{K}_{j}^{I}$ uniformly converging to $x(\cdot) \in \mathcal{K}_{j}^{I}$, then

$$
\lim _{n \rightarrow \infty} \int_{I}\left|f\left(t, x_{n}(t)\right)-f(t, x(t))\right|^{p} d t=0 .
$$

- We say that a function $f: \mathbb{R} \times \mathbb{R}^{N} \rightarrow \mathbb{R}^{M}$ is weak $\Theta$-Carathéodory, and write $f \in \mathfrak{W} \Theta \mathfrak{C}\left(\mathbb{R}^{M}\right)$ (or simply $f \in \mathfrak{W} \Theta \mathfrak{C}$ when $M=N$ ), if $f$ satisfies (C1), (C2) and

(W) for each $j \in \mathbb{N}$ and $I=\left[q_{1}, q_{2}\right], q_{1}, q_{2} \in \mathbb{Q}$, if $\left(x_{n}(\cdot)\right)_{n \in \mathbb{N}}$ is a sequence in $\mathcal{K}_{j}^{I}$ uniformly converging to $x(\cdot) \in \mathcal{K}_{j}^{I}$, then

$$
\lim _{n \rightarrow \infty} \int_{I} f\left(t, x_{n}(t)\right) d t=\int_{I} f(t, x(t)) d t .
$$

Remark 2.5. As regards Definitions 2.1, 2.2 and 2.4, when $p=1$, we will omit the number 1 from the notation. For example, we will simply write $\mathfrak{L} \mathfrak{C}$ instead of $\mathfrak{L} \mathfrak{C}_{1}$.

As a first topological result we give a characterization of the functions in $\Theta \mathfrak{C}_{p}\left(\mathbb{R}^{M}\right)$ and in $\mathfrak{W} \Theta \mathfrak{C}_{p}\left(\mathbb{R}^{M}\right)$.

Theorem 2.6. Let $f$ be a function in $\mathfrak{C}_{p}\left(\mathbb{R}^{M}\right)$.

(i) If there exists a sequence $\left(f_{n}\right)_{n \in \mathbb{N}}$ in $\Theta \mathfrak{C}\left(\mathbb{R}^{M}\right)$ such that for every $\mathcal{K}_{j}^{I}$, as in Definition 2.4, one has

$$
\lim _{n \rightarrow \infty} \sup _{y(\cdot) \in \mathcal{K}_{j}^{I}} \int_{I}\left|f_{n}(t, y(t))-f(t, y(t))\right|^{p} d t=0,
$$

then $f \in \Theta \mathfrak{C}\left(\mathbb{R}^{M}\right)$.

(ii) If there exists a sequence $\left(f_{n}\right)_{n \in \mathbb{N}}$ in $\mathfrak{W} \Theta \mathfrak{C}\left(\mathbb{R}^{M}\right)$ such that for every $\mathcal{K}_{j}^{I}$, as in Definition 2.4, one has

$$
\lim _{n \rightarrow \infty} \sup _{y(\cdot) \in \mathcal{K}_{j}^{I}}\left|\int_{I}\left[f_{n}(t, y(t))-f(t, y(t))\right] d t\right|=0,
$$

then $f \in \mathfrak{W} \Theta \mathfrak{C}\left(\mathbb{R}^{M}\right)$. 
Proof. (i). Since condition (C1) and (C2) are satisfied by hypothesis, we only need to prove condition (T). Consider $I=\left[q_{1}, q_{2}\right], q_{1}, q_{2} \in \mathbb{Q}, j \in \mathbb{N}$, and let $\left(x_{k}(\cdot)\right)_{k \in \mathbb{N}}$ be a sequence in $\mathcal{K}_{j}^{I}$ converging uniformly to some $x(\cdot) \in \mathcal{K}_{j}^{I}$. Thanks to equation (2.3), for a fixed $\varepsilon>0$ there exists $n_{0} \in \mathbb{N}$ such that,

$$
\sup _{y(\cdot) \in \mathcal{K}_{j}^{I}}\left[\int_{I}\left|f_{n_{0}}(t, y(t))-f(t, y(t))\right|^{p} d t\right]^{1 / p}<\frac{\varepsilon}{2} .
$$

Therefore, we have that

$$
\begin{aligned}
\| f\left(\cdot, x_{k}(\cdot)\right) & -f(\cdot, x(\cdot))\left\|_{p} \leq\right\| f\left(\cdot, x_{k}(\cdot)\right)-f_{n_{0}}\left(\cdot, x_{k}(\cdot)\right) \|_{p} \\
& +\left\|f_{n_{0}}\left(\cdot, x_{k}(\cdot)\right)-f_{n_{0}}(\cdot, x(\cdot))\right\|_{p}+\left\|f(\cdot, x(\cdot))-f_{n_{0}}(\cdot, x(\cdot))\right\|_{p} \\
\leq & \varepsilon+\left\|f_{n_{0}}\left(\cdot, x_{k}(\cdot)\right)-f_{n_{0}}(\cdot, x(\cdot))\right\|_{p} .
\end{aligned}
$$

Then, recalling that $f_{n_{0}} \in \Theta \mathfrak{C}\left(\mathbb{R}^{M}\right)$, from ( $\left.\mathbf{T}\right)$ and (2.4), we conclude that

$$
\lim _{k \rightarrow \infty} \int_{I}\left|f\left(t, x_{k}(t)\right)-f(t, x(t))\right|^{p} d t=0
$$

and condition (T) holds for $f$.

(ii). Analogous reasonings lead to the thesis also for $\mathfrak{W} \Theta \mathfrak{C}\left(\mathbb{R}^{M}\right)$.

Remark 2.7. We identify the functions which lay in the same set and only differ on a negligible subset of $\mathbb{R}^{1+N}$. The constraint about belonging to the same set is crucial. Otherwise, a function in $\mathfrak{S C}_{p}\left(\mathbb{R}^{M}\right)$ could in fact be identified with a function which is not in $\mathfrak{S C}_{p}\left(\mathbb{R}^{M}\right)$. Furthermore, such a rule implies that $\mathfrak{L C}_{p}\left(\mathbb{R}^{M}\right) \subset \mathfrak{S C}_{p}\left(\mathbb{R}^{M}\right)$ and $\Theta \mathfrak{C}_{p}\left(\mathbb{R}^{M}\right) \subseteq \mathfrak{W} \Theta \mathfrak{C}\left(\mathbb{R}^{M}\right)$ but $\mathfrak{S C}_{p}\left(\mathbb{R}^{M}\right)$ is not included in $\Theta \mathfrak{C}_{p}\left(\mathbb{R}^{M}\right)$. Nevertheless, a continuous injection (which is not a bijection) of $\mathfrak{S C}_{p}\left(\mathbb{R}^{M}\right)$ in $\Theta \mathfrak{C}_{p}\left(\mathbb{R}^{M}\right)$ is straightforward. Thus, the following chain can be sketched

$$
\mathfrak{L} \mathfrak{C}_{p}\left(\mathbb{R}^{M}\right) \subset \mathfrak{S C}_{p}\left(\mathbb{R}^{M}\right) \hookrightarrow \Theta \mathfrak{C}_{p}\left(\mathbb{R}^{M}\right) \subseteq \mathfrak{W} \Theta \mathfrak{C}\left(\mathbb{R}^{M}\right)
$$

where $\Theta$ is any suitable set of moduli of continuity. In particular, for any function $f \in \mathfrak{W} \Theta \mathfrak{C}\left(\mathbb{R}^{M}\right)$, we will say that $f \in \mathfrak{S C}_{p}\left(\mathbb{R}^{M}\right)$ (resp. $f \in \mathfrak{L C}_{p}\left(\mathbb{R}^{M}\right)$ ) if $f$ is a representative of an equivalence class in $\mathfrak{W} \Theta \mathfrak{C}\left(\mathbb{R}^{M}\right)$ in which there is at least one function that belongs to $\mathfrak{S C}_{p}\left(\mathbb{R}^{M}\right)$ (resp. $\mathfrak{L C}_{p}\left(\mathbb{R}^{M}\right)$ ). On the other hand, the equivalence class in $\mathfrak{W} \Theta \mathfrak{C}\left(\mathbb{R}^{M}\right)$ of a function $f \in \mathfrak{S C}_{p}\left(\mathbb{R}^{M}\right)$, contains elements which do not belong to the equivalence class in $\mathfrak{S C}_{p}\left(\mathbb{R}^{M}\right)$ of $f$.

The following results characterizes the process of identification in the sets $\mathfrak{W} \Theta \mathfrak{C}\left(\mathbb{R}^{M}\right)$ and $\Theta \mathfrak{C}_{p}\left(\mathbb{R}^{M}\right)$, and, as a consequence, implies that $\mathfrak{W} \Theta \mathfrak{C}\left(\mathbb{R}^{M}\right)$ and $\Theta \mathfrak{C}_{p}\left(\mathbb{R}^{M}\right)$ are metric spaces when endowed with the topologies defined at the beginning of the following section. 
Proposition 2.8. Let $f, g \in \mathfrak{W} \Theta \mathfrak{C}\left(\mathbb{R}^{M}\right)$ (resp. $f, g \in \Theta \mathfrak{C}_{p}\left(\mathbb{R}^{M}\right)$ ) coincide almost everywhere in $\mathbb{R} \times \mathbb{R}^{N}$. Then, for any $\mathcal{K}_{j}^{I}$ as in Definition 2.4 , we have that

$$
x(\cdot) \in \mathcal{K}_{j}^{I} \quad \Rightarrow \quad f(t, x(t))=g(t, x(t)) \text { for a.e. } t \in I .
$$

Proof. Consider $f, g \in \mathfrak{W} \Theta \mathfrak{C}\left(\mathbb{R}^{M}\right)$, and the Borel set $V \subset \mathbb{R} \times \mathbb{R}^{N}$, such that

$$
f(t, x)=g(t, x) \quad \forall(t, x) \in V \quad \text { and } \quad \operatorname{meas}_{\mathbb{R}^{1+N}}\left(\mathbb{R}^{1+N} \backslash V\right)=0 .
$$

Fix $j \in \mathbb{N}, I=\left[q_{1}, q_{2}\right], q_{1}, q_{2} \in \mathbb{Q}$, and $x(\cdot) \in \mathcal{K}_{j}^{I}$, and consider the set

$$
E=\left\{(t, \varepsilon) \in I \times B_{1} \subset \mathbb{R}^{1+N} \mid(t, x(t)+\varepsilon) \in V\right\} .
$$

Moreover, for any $t \in I$ denote by $E_{t}$ and by $V_{t}$, the sections in $t$ of $E$ and $V$, respectively, i.e.

$$
E_{t}=\left\{\varepsilon \in B_{1} \mid(t, \varepsilon) \in E\right\} \quad \text { and } \quad V_{t}=\left\{x \in \mathbb{R}^{N} \mid(t, x) \in V\right\}
$$

Now, for a given $t \in I$ one has

$$
x(t)+\left(B_{1} \backslash E_{t}\right) \subset B_{j+1} \backslash V_{t} .
$$

Therefore, $\operatorname{meas}_{\mathbb{R}^{N}}\left(B_{1} \backslash E_{t}\right)=0$ for almost every $t \in I$. Then, applying Fubini's theorem twice, one has

$$
\operatorname{meas}_{\mathbb{R}}(I) \cdot \operatorname{meas}_{\mathbb{R}^{N}}\left(B_{1}\right)=\operatorname{meas}_{\mathbb{R}^{1+N}}(E)=\int_{\mathbb{R}^{N}} \operatorname{meas}_{\mathbb{R}}\left(E_{\varepsilon}\right) d \varepsilon
$$

where $E_{\varepsilon}$ denotes the section of $E$ for any fixed $\varepsilon \in B_{1}$, i.e.

$$
E_{\varepsilon}=\{t \in I \mid(t, \varepsilon) \in E\}
$$

Therefore, we have that $\operatorname{meas}_{\mathbb{R}}\left(E_{\varepsilon}\right)=\operatorname{meas}_{\mathbb{R}}(I)$ for almost every $\varepsilon \in B_{1}$. Now, let $\left(\varepsilon_{n}\right)_{n \in \mathbb{N}} \subset B_{1}$ be such that

$$
\varepsilon_{n} \stackrel{n \rightarrow \infty}{\longrightarrow} 0 \quad \text { and } \quad \operatorname{meas}_{\mathbb{R}}\left(E_{\varepsilon_{n}}\right)=\operatorname{meas}_{\mathbb{R}}(I) \quad \forall n \in \mathbb{N}
$$

Then, for any $n \in \mathbb{N}$, called $x_{n}(t)=x(t)+\varepsilon_{n}$, one has that $x_{n}(\cdot) \in \mathcal{K}_{j+1}^{I}$, and $f\left(t, x_{n}(t)\right)=g\left(t, x_{n}(t)\right)$ for all $t \in I$. Then,

$$
\begin{aligned}
& \left|\int_{I}[f(t, x(t))-g(t, x(t))] d t\right| \leq\left|\int_{I}\left[f(t, x(t))-f\left(t, x_{n}(t)\right)\right] d t\right| \\
& \quad+\left|\int_{I}\left[f\left(t, x_{n}(t)\right)-g\left(t, x_{n}(t)\right)\right] d t\right|+\left|\int_{I}\left[g\left(t, x_{n}(t)\right)-g(t, x(t))\right] d t\right| \\
& =\left|\int_{I}\left[f(t, x(t))-f\left(t, x_{n}(t)\right)\right] d t\right|+\left|\int_{I}\left[g\left(t, x_{n}(t)\right)-g(t, x(t))\right] d t\right|
\end{aligned}
$$


and the terms on the right-hand side go to zero as $n \rightarrow \infty$ because $f, g \in$ $\mathfrak{W} \Theta \mathfrak{C}\left(\mathbb{R}^{M}\right)$. In other words, we have that for any $j \in \mathbb{N}, I=\left[q_{1}, q_{2}\right], q_{1}, q_{2} \in \mathbb{Q}$

$$
x(\cdot) \in \mathcal{K}_{j}^{I} \Rightarrow \int_{I} f(t, x(t)) d t=\int_{I} g(t, x(t)) d t .
$$

However, as a first step to obtain (2.6), we would need that for every $t \in I$ and $h>0$ so that $t+h \in I$ one has

$$
\int_{t}^{t+h} f(t, x(t)) d t=\int_{t}^{t+h} g(t, x(t)) d t
$$

which can not be directly deduced from (2.7), nor reasoning as to obtain (2.7) because if a function $x(\cdot)$ belongs to the set $\mathcal{K}_{j}^{I}$, and we take $p_{1}, p_{2} \in \mathbb{Q}$ such that $J=\left[p_{1}, p_{2}\right] \subset I$, then $x(\cdot)$ does not necessarily belong to $\mathcal{K}_{j}^{J}$. In fact, due to the relation of partial order in a set of moduli of continuity (see Definition 2.3), it might happen that for any $t \in \mathbb{R} \backslash\{0\}$ one has $\theta_{j}^{J}(t)<\theta_{j}^{I}(t)$, where $\theta_{j}^{J}(\cdot)$ and $\theta_{j}^{I}(\cdot)$ are the moduli of continuity on $\mathcal{K}_{j}^{J}$ and $\mathcal{K}_{j}^{I}$, respectively, and that $\theta_{j}^{J}(\cdot)$ is not a modulus of continuity for the restriction of $x(\cdot)$ to the interval $J$. Nevertheless, we can proceed as follows: fix $p_{1}, p_{2} \in \mathbb{Q}$ such that $q_{1} \leq p_{1}<p_{2} \leq q_{2}$ and consider the function $\widehat{x}: I \rightarrow \mathbb{R}^{N}$ defined by

$$
\widehat{x}(t)= \begin{cases}x\left(p_{1}\right) & \text { if } t \in\left[q_{1}, p_{1}\right], \\ x(t) & \text { if } t \in\left[p_{1}, p_{2}\right], \\ x\left(p_{2}\right) & \text { if } t \in\left[p_{2}, q_{2}\right] .\end{cases}
$$

Clearly, $\widehat{x}(\cdot)$ is still a function in $\mathcal{K}_{j}^{I}$. Then, using the linearity of the integral and the triangular inequality, one has

$$
\begin{aligned}
& \left|\int_{p_{1}}^{p_{2}}[f(t, x(t))-g(t, x(t))] d t\right| \leq\left|\int_{q_{1}}^{q_{2}}[f(t, \widehat{x}(t))-g(t, \widehat{x}(t))] d t\right|+ \\
& \quad+\left|\int_{q_{1}}^{p_{1}}\left[f\left(t, x\left(p_{1}\right)\right)-g\left(t, x\left(p_{1}\right)\right)\right] d t\right|+\left|\int_{p_{2}}^{q_{2}}\left[f\left(t, x\left(p_{2}\right)\right)-g\left(t, x\left(p_{2}\right)\right)\right] d t\right|,
\end{aligned}
$$

and each one of the three integrals on the right-hand side is equal to zero due to (2.7) applied to $\widehat{x}\left(p_{1}\right) \in \mathcal{K}_{j}^{I}, x(\cdot) \in \mathcal{K}_{j}^{\left[q_{1}, p_{1}\right]}$ and $x\left(p_{2}\right) \in \mathcal{K}_{j}^{\left[p_{2}, q_{2}\right]}$, respectively. In particular, we obtain (2.8) for any $t \in I$ and $h>0$ so that $t+h \in I$, thanks to the continuity of the integral with respect to the interval of integration.

Thus, dividing both the sides in (2.8) by $h$, and taking the limit as $h \rightarrow 0$ (see N. Dunford and J.T. Schwartz [24, Corollary III.12.7, p.216]) one obtains that

$$
f(t, x(t))=g(t, x(t)) \quad \text { for a.e. } t \in \mathbb{R} .
$$

If $f, g \in \Theta \mathfrak{C}_{p}\left(\mathbb{R}^{M}\right)$, and $p>1$, one has the thesis recalling that $L_{l o c}^{p} \subset L_{l o c}^{1}$. 


\subsection{Topologies}

We endow the previously introduced sets with suitable topologies. As a rule, when inducing a topology on a subspace we will denote the induced topology with the same symbol which denotes the topology on the original space.

The space $\mathfrak{W} \Theta \mathfrak{C}\left(\mathbb{R}^{M}\right)$ will be endowed with the following weak topology.

Definition 2.9 (Topology $\sigma_{\Theta}$ ). Let $\Theta$ be a suitable set of moduli of continuity as in Definition 2.3. We call $\sigma_{\Theta}$ the topology on $\mathfrak{W} \Theta \mathfrak{C}\left(\mathbb{R}^{M}\right)$ generated by the family of seminorms

$$
p_{I, j}(f)=\sup _{x(\cdot) \in \mathcal{K}_{j}^{I}}\left|\int_{I} f(t, x(t)) d t\right|, \quad f \in \mathfrak{W} \Theta \mathfrak{C}\left(\mathbb{R}^{M}\right),
$$

with $I=\left[q_{1}, q_{2}\right], q_{1}, q_{2} \in \mathbb{Q}, j \in \mathbb{N}$, and $\mathcal{K}_{j}^{I}$ as in Definition 2.4. The space $\left(\mathfrak{W} \Theta \mathfrak{C}\left(\mathbb{R}^{M}\right), \sigma_{\Theta}\right)$ is a locally convex metric space.

The topology $\sigma_{\Theta}$ can also be induced on the spaces $\Theta \mathfrak{C}\left(\mathbb{R}^{M}\right), \mathfrak{S C}\left(\mathbb{R}^{M}\right)$ and $\mathfrak{L} \mathfrak{C}\left(\mathbb{R}^{M}\right)$. Moreover, the space $\Theta \mathfrak{C}_{p}\left(\mathbb{R}^{M}\right)$ will be endowed with the following strong topology.

Definition 2.10 (Topology $\mathcal{T}_{\Theta}$ ). Let $\Theta$ be a suitable set of moduli of continuity as in Definition 2.3. We call $\mathcal{T}_{\Theta}$ the topology on $\Theta \mathfrak{C}_{p}\left(\mathbb{R}^{M}\right)$ generated by the family of seminorms

$$
p_{I, j}(f)=\sup _{x(\cdot) \in \mathcal{K}_{j}^{I}}\left[\int_{I}|f(t, x(t))|^{p} d t\right]^{1 / p}, \quad f \in \Theta \mathfrak{C}\left(\mathbb{R}^{M}\right),
$$

with $I=\left[q_{1}, q_{2}\right], q_{1}, q_{2} \in \mathbb{Q}, j \in \mathbb{N}$, and $\mathcal{K}_{j}^{I}$ as in Definition 2.4. The space $\left(\Theta \mathfrak{C}_{p}\left(\mathbb{R}^{M}\right), \mathcal{T}_{\Theta}\right)$ is a locally convex metric space.

Remark 2.11. Notice that, according to the previous definition, one actually has a different topology $\mathcal{T}_{\Theta}=\mathcal{T}_{\Theta}(p)$ for any $1 \leq p<\infty$. However, in order to keep the notation simple, we will simply write $\mathcal{T}_{\Theta}$ since in each case it is always clear which $p$ we are using because it is specified in the notation of the space (e.g. $\left.\left(\Theta \mathfrak{C}_{p}\left(\mathbb{R}^{M}\right), \mathcal{T}_{\Theta}\right)\right)$. The same reasoning also applies to the rest of the topologies that we will define in this chapter.

Besides the already introduced topologies $\sigma_{\Theta}$ and $\mathcal{T}_{\Theta}$, on the set $\mathfrak{S C}_{p}\left(\mathbb{R}^{M}\right)$ we also consider the following three topologies which have been previously used in the literature.

Definition 2.12 (Topology $\left.\mathcal{T}_{B}\right)$. We call $\mathcal{T}_{B}$ the topology on $\mathfrak{S C}_{p}\left(\mathbb{R}^{M}\right)$ generated by the family of seminorms

$$
p_{I, j}(f)=\sup _{x(\cdot) \in C\left(I, B_{j}\right)}\left[\int_{I}|f(t, x(t))|^{p} d t\right]^{1 / p}, \quad f \in \mathfrak{S C}_{p}\left(\mathbb{R}^{M}\right),
$$

where $I=\left[q_{1}, q_{2}\right], q_{1}, q_{2} \in \mathbb{Q}$ and $j \in \mathbb{N} . \quad\left(\mathfrak{S C}_{p}\left(\mathbb{R}^{M}\right), \mathcal{T}_{B}\right)$ is a locally convex metric space. 
Definition 2.13 (Topologies $\mathcal{T}_{D}$ and $\sigma_{D}$ ). Let $D$ be a countable and dense subset of $\mathbb{R}^{N}$.

- We call $\mathcal{T}_{D}$ the topology on $\mathfrak{S C}_{p}\left(\mathbb{R}^{M}\right)$ generated by the family of seminorms

$$
p_{I, x_{j}}(f)=\left[\int_{I}\left|f\left(t, x_{j}\right)\right|^{p} d t\right]^{1 / p}
$$

for $f \in \mathfrak{S C}_{p}\left(\mathbb{R}^{M}\right), x_{j} \in D, I=\left[q_{1}, q_{2}\right], q_{1}, q_{2} \in \mathbb{Q}$.

- We call $\sigma_{D}$ the topology on $\mathfrak{S} \mathfrak{C}\left(\mathbb{R}^{M}\right)$ generated by the family of seminorms

$$
p_{I, x}(f)=\left|\int_{I} f(t, x) d t\right|
$$

for $f \in \mathfrak{S C}\left(\mathbb{R}^{M}\right), x \in D, I=\left[q_{1}, q_{2}\right], q_{1}, q_{2} \in \mathbb{Q}$.

$\left(\mathfrak{S C}_{p}\left(\mathbb{R}^{M}\right), \mathcal{T}_{D}\right)$ and $\left(\mathfrak{S C}\left(\mathbb{R}^{M}\right), \sigma_{D}\right)$ are locally convex metric spaces.

Notice that, as well as $\mathfrak{S C}_{p}\left(\mathbb{R}^{M}\right)$, also $\mathfrak{L} \mathfrak{C}_{p}\left(\mathbb{R}^{M}\right)$ can be endowed with all the previous topologies and the following chains of order hold when all the previous topologies are considered in either $\mathfrak{S C}_{p}\left(\mathbb{R}^{M}\right)$ or $\mathfrak{L C}_{p}\left(\mathbb{R}^{M}\right)$ :

$$
\sigma_{D} \leq \mathcal{T}_{D} \leq \mathcal{T}_{\Theta} \leq \mathcal{T}_{B} \quad \text { and } \quad \sigma_{D} \leq \sigma_{\Theta} \leq \mathcal{T}_{\Theta}
$$

We conclude this section with a technical lemma on $\left(\mathfrak{W} \Theta \mathfrak{C}\left(\mathbb{R}^{M}\right), \sigma_{\Theta}\right)$. As one may notice, both the property $(\mathbf{W})$ in Definition 2.4 and the way the topology $\sigma_{\Theta}$ is defined in Definition 2.9, require that the interval on which we take the integral coincides with the domain of the functions over which we take the supremum. However, in many cases we will need to consider the integral on a smaller subinterval that we can not directly control with the integral on the whole interval due to the employed weak formulation. Nevertheless, playing with the functions in the compact sets $\mathcal{K}_{j}^{J}$ (on the line of what we already did in the proof of Proposition 2.8), we are still able to achieve the properties we need, as shown in the technical lemma below.

Lemma 2.14. Let $\Theta$ be a suitable set of moduli of continuity as in Definition 2.3, and for each $j \in \mathbb{N}$ and $I=\left[q_{1}, q_{2}\right], q_{1}, q_{2} \in \mathbb{Q}$, let $\mathcal{K}_{j}^{I}$ be the compact set in $C\left(I, B_{j}\right)$ defined as in Definition 2.4.

(i) Let $f$ be a function of $\mathfrak{W} \Theta \mathfrak{C}\left(\mathbb{R}^{M}\right)$. For each $j \in \mathbb{N}$ and $I=\left[q_{1}, q_{2}\right], q_{1}, q_{2} \in \mathbb{Q}$, if $\left(x_{n}(\cdot)\right)_{n \in \mathbb{N}}$ is a sequence in $\mathcal{K}_{j}^{I}$ uniformly converging to $x(\cdot) \in \mathcal{K}_{j}^{I}$, then

$$
\lim _{n \rightarrow \infty} \int_{p_{1}}^{p_{2}} f\left(t, x_{n}(t)\right) d t=\int_{p_{1}}^{p_{2}} f(t, x(t)) d t,
$$

whenever $p_{1}, p_{2} \in \mathbb{Q}$ and $q_{1} \leq p_{1}<p_{2} \leq q_{2}$. 
(ii) Let $\left(g_{n}\right)_{n \in \mathbb{N}}$ be a sequence in $\mathfrak{W} \Theta \mathfrak{C}\left(\mathbb{R}^{M}\right)$ converging to $g$ in $\left(\mathfrak{W} \Theta \mathfrak{C}\left(\mathbb{R}^{M}\right), \sigma_{\Theta}\right)$. Then, for any $I=\left[q_{1}, q_{2}\right], q_{1}, q_{2} \in \mathbb{Q}$ and $j \in \mathbb{N}$ one has

$$
\lim _{n \rightarrow \infty} \sup _{x(\cdot) \in \mathcal{K}_{j}^{I}}\left|\int_{p_{1}}^{p_{2}}\left[g_{n}(t, x(t))-g(t, x(t))\right] d t\right|=0
$$

whenever $p_{1}, p_{2} \in \mathbb{Q}$ and $q_{1} \leq p_{1}<p_{2} \leq q_{2}$.

Proof. (i). Consider $I=\left[q_{1}, q_{2}\right]$, with $q_{1}, q_{2} \in \mathbb{Q}$, and a sequence $\left(x_{n}(\cdot)\right)_{n \in \mathbb{N}}$ in $\mathcal{K}_{j}^{I}$ uniformly converging to a function $x(\cdot) \in \mathcal{K}_{j}^{I}$. For every $n \in \mathbb{N}$, consider the function $\widehat{x}_{n}: I \rightarrow B_{j}$ defined by

$$
\widehat{x}_{n}(t)= \begin{cases}x_{n}\left(p_{1}\right) & \text { if } t \in\left[q_{1}, p_{1}\right] \\ x_{n}(t) & \text { if } t \in\left[p_{1}, p_{2}\right] \\ x_{n}\left(p_{2}\right) & \text { if } t \in\left[p_{2}, q_{2}\right]\end{cases}
$$

Notice that for all $n \in \mathbb{N}$ one can write

$$
\int_{p_{1}}^{p_{2}} f\left(t, x_{n}(t)\right) d t=\int_{q_{1}}^{q_{2}} f\left(t, \widehat{x}_{n}(t)\right) d t-\int_{q_{1}}^{p_{1}} f\left(t, x_{n}\left(p_{1}\right)\right) d t-\int_{p_{2}}^{q_{2}} f\left(s, x_{n}\left(p_{2}\right)\right) d t .
$$

The sequence $\left(\widehat{x}_{n}(\cdot)\right)_{n \in \mathbb{N}}$ is in $\mathcal{K}_{j}^{I}$ and converges uniformly to the function $\widehat{x}: I \rightarrow$ $\mathbb{R}^{N}$ which coincides with $x(\cdot)$ on $\left[p_{1}, p_{2}\right]$, and it has constant value $x\left(p_{1}\right)$ and $x\left(p_{2}\right)$ on $\left[q_{1}, p_{1}\right]$ and $\left[p_{2}, q_{2}\right]$, respectively. In particular, notice that $\left(x_{n}\left(p_{1}\right)\right)_{n \in \mathbb{N}}$ is a sequence in $\mathcal{K}_{j}^{\left[q_{1}, p_{1}\right]}$ and $\left(x_{n}\left(p_{2}\right)\right)_{n \in \mathbb{N}}$ is a sequence in $\mathcal{K}_{j}^{\left[p_{2}, q_{2}\right]}$, since both are sequences of constants bounded by $j$, and they respectively converge to the constant functions given by $x\left(p_{1}\right) \in \mathcal{K}_{j}^{\left[q_{1}, p_{1}\right]}$ and $x\left(p_{2}\right) \in \mathcal{K}_{j}^{\left[p_{2}, q_{2}\right]}$. Therefore, statement (i) follows from (2.11) and $(\mathbf{W})$.

(ii). Consider a sequence $\left(g_{n}\right)_{n \in \mathbb{N}}$ in $\mathfrak{W} \Theta \mathfrak{C}\left(\mathbb{R}^{M}\right)$ converging to a function $g$ in $\left(\mathfrak{W} \Theta \mathfrak{C}\left(\mathbb{R}^{M}\right), \sigma_{\Theta}\right)$, a function $x(\cdot) \in \mathcal{K}_{j}^{I}$, where $I=\left[q_{1}, q_{2}\right], q_{1}, q_{2} \in \mathbb{Q}$, and let $p_{1}, p_{2}$ be elements of $\mathbb{Q}$ such that $q_{1} \leq p_{1}<p_{2} \leq q_{2}$. Consider the function $\widehat{x}: I \rightarrow \mathbb{R}^{N}$ defined as in (2.9).

Clearly, $\widehat{x}$ is still a function in $\mathcal{K}_{j}^{I}$. Recalling how $\widehat{x}$ has been defined in the previous formula, using the linearity of the integral and the triangular inequality, one has

$$
\begin{aligned}
& \left|\int_{p_{1}}^{p_{2}}\left[g_{n}(t, x(t))-g(t, x(t))\right] d t\right| \leq\left|\int_{q_{1}}^{q_{2}}\left[g_{n}(t, \widehat{x}(t))-g(t, \widehat{x}(t))\right] d t\right|+ \\
& \quad+\left|\int_{q_{1}}^{p_{1}}\left[g_{n}\left(t, x\left(p_{1}\right)\right)-g\left(t, x\left(p_{1}\right)\right)\right] d t\right|+\left|\int_{p_{2}}^{q_{2}}\left[g_{n}\left(t, x\left(p_{2}\right)\right)-g\left(t, x\left(p_{2}\right)\right)\right] d t\right| .
\end{aligned}
$$

Moreover, since any constant function bounded by $j$ belongs to $\mathcal{K}_{j}^{J}$ for any interval 
$J \subset \mathbb{R}$ with rational extrema, then from the previous inequality we can deduce

$$
\begin{aligned}
\left|\int_{p_{1}}^{p_{2}}\left[g_{n}(t, x(t))-g(t, x(t))\right] d t\right| \leq & \sup _{x(\cdot) \in \mathcal{K}_{j}^{I}}\left|\int_{q_{1}}^{q_{2}}\left[g_{n}(t, x(t))-g(t, x(t))\right] d t\right| \\
& +\sup _{x(\cdot) \in \mathcal{K}_{j}^{\left[q_{1}, p_{1}\right]} \mid}\left|\int_{q_{1}}^{p_{1}}\left[g_{n}(t, x(t))-g(t, x(t))\right] d t\right| \\
& +\sup _{x(\cdot) \in \mathcal{K}_{j}^{\left[p_{2}, q_{2}\right]}}\left|\int_{p_{2}}^{q_{2}}\left[g_{n}(t, x(t))-g(t, x(t))\right] d t\right|
\end{aligned}
$$

and the right-hand side of the previous inequality goes to zero as $n \rightarrow \infty$ since $\left(g_{n}\right)_{n \in \mathbb{N}}$ converges to $g$ in $\left(\mathfrak{W} \Theta \mathfrak{C}\left(\mathbb{R}^{M}\right), \sigma_{\Theta}\right)$. In particular notice that such limit is uniform in $x(\cdot) \in \mathcal{K}_{j}^{I}$ and thus one obtains the thesis.

Remark 2.15. Notice that properties (i) and (ii) of Lemma 2.14 are trivially true for any strong topology. Indeed, the modulus inside the integral allows us to control any integral with one on a bigger interval in which we are sure to have the convergence.

\subsection{The $m$-bounds and the $l$-bounds}

This section is devoted to a deeper understanding of the $m$-bounds and $l$-bounds of a Carathéodory function or set of functions. Interestingly, the role of these locally integrable functions goes far beyond the existence and uniqueness of solutions for Carathéodory differential problems as stated in Theorem 1.2. However, in order to proceed in the development of the theory, we need to give a bit of structure to them in a way that different Carathéodory functions can be related to each other through their $m$-bounds and $l$-bounds. To the aim, we introduce the notions of $L_{l o c}^{1}$-equicontinuity and $L_{l o c}^{p}$-boundedness and subsequently relate them to Carathéodory functions through their $m$-bounds and $l$-bounds.

Definition 2.16 ( $L_{l o c}^{p}$-boundedness). A subset $S$ of positive functions in $L_{l o c}^{p}$, with $1 \leq p<\infty$ is bounded if for every $r>0$ the following inequality holds

$$
\sup _{m \in S} \int_{-r}^{r} m^{p}(t) d t<\infty
$$

In such a case we will say that $S$ is $L_{l o c}^{p}$-bounded.

Definition 2.17 ( $L_{l o c}^{1}$-equicontinuity). A set $S$ of positive functions in $L_{l o c}^{1}$ is $L_{l o c}^{1}$-equicontinuous if for any $r>0$ and for any $\varepsilon>0$ there exists a $\delta=\delta(r, \varepsilon)>0$ such that, for any $-r \leq s \leq t \leq r$, we have

$$
t-s<\delta \Rightarrow \sup _{m \in S} \int_{s}^{t} m(u) d u<\varepsilon .
$$


Remark 2.18. According to the previous definitions, the $L_{l o c}^{1}$-equicontinuity implies the $L_{l o c}^{1}$-boundedness. On the other hand, if $p>1$, then, thanks to Hölder inequality one immediately obtains that the $L_{l o c}^{p}$-boundedness implies the $L_{l o c^{-}}^{1}$ equicontinuity.

As follows, we aim to give a characterization of the $L_{l o c}^{1}$-equicontinuous subsets of positive functions in $L_{l o c}^{1}$ through the relative compactness of the associated set of positive absolutely continuous measures. In order to proceed, we need to set some notation and give some statements of classic results of measure theory. By $\mathcal{M}^{+}$we denote the set of locally finite, positive and regular Borel measures on $\mathbb{R}$. Recall that a measure $\mu \in \mathcal{M}^{+}$is said to be continuous if $\mu(t)=0$ for any $t \in \mathbb{R}$. Additionally, we denote by $\mathcal{M}_{a c}^{+}$the set of measures $\mu \in \mathcal{M}^{+}$such that for every $r \in \mathbb{R}^{+}$the restriction of $\mu$ to the interval $[-r, r]$ is absolutely continuous with respect to the Lebesgue measure, i.e. such that, for any Lebesgue measurable set $A \subset \mathbb{R}$, one has

$$
\operatorname{meas}_{\mathbb{R}}(A)=0 \quad \Rightarrow \quad \mu(A)=0,
$$

where meas $_{\mathbb{R}}$ represents the Lebesgue measure on $\mathbb{R}$. By the Lebesgue-RadonNikodým theorem, if $\mu \in \mathcal{M}_{a c}^{+}$, then, there exists $f \in L_{l o c}^{1}$ such that for any measurable set $A$ in $\mathbb{R}$, one has

$$
\mu(A)=\int_{A} f(t) d \operatorname{meas}_{\mathbb{R}}(t)=\int_{A} f(t) d t .
$$

If a measure $\mu \in \mathcal{M}^{+}$is not absolutely continuous with respect to the Lebesgue measure, then it is said to be singular. In particular, we will denote by $\mathcal{M}_{s c}^{+}$the set of singular continuous measures, i.e. positive and regular Borel measures which are continuous and singular with respect to the Lebesgue measure, and by $\mathcal{M}_{p d}^{+}$, the set of purely discontinuous measures, i.e. positive and regular Borel measures with support on a countable subset of $\mathbb{R}$. The set $\mathcal{M}^{+}$can be decomposed as $\mathcal{M}^{+}=\mathcal{M}_{a c}^{+} \oplus \mathcal{M}_{s c}^{+} \oplus \mathcal{M}_{p d}^{+}$(see Hewitt and Stromberg [31, Theorem 19.61, p.337]).

We endow $\mathcal{M}^{+}$with the following topology.

Definition 2.19 (Vague topology). We say that a sequence $\left(\mu_{n}\right)_{n \in \mathbb{N}}$ of measures in $\mathcal{M}^{+}$vaguely converges to $\mu \in \mathcal{M}^{+}$, and write $\mu_{n} \stackrel{\widetilde{\sigma}}{\rightarrow} \mu$, if and only if

$$
\lim _{n \rightarrow \infty} \int_{\mathbb{R}} \phi(s) d \mu_{n}(s)=\int_{\mathbb{R}} \phi(s) d \mu(s) \quad \text { for all } \phi \in C_{C}^{+}(\mathbb{R}) .
$$

We will denote such a topological space by $\left(\mathcal{M}^{+}, \tilde{\sigma}\right)$.

As shown in Kallenberg [35, Theorem 15.7.7, p.170], $\left(\mathcal{M}^{+}, \widetilde{\sigma}\right)$ is a Polish space, i.e. it is separable and completely metrizable. Moreover, the following propositions hold true (see [35, Theorems 15.7.5, p.170 and 15.7.2, p.169]).

Proposition 2.20. Any subset $M \subset \mathcal{M}^{+}$is relatively compact in the vague topology if and only if $\sup _{\mu \in M} \mu(B)<\infty$ for any bounded Borel set $B$. 
Remark 2.21. As a consequence of the previous proposition, if $S$ is a subset of $L_{l o c}^{1}$, and $M \subset \mathcal{M}_{a c}^{+}$is the set of positive absolutely continuous measures whose densities are the functions in $S$, then $S$ is $L_{l o c}^{1}$-bounded if and only if $M$ is relatively compact in the vague topology.

Proposition 2.22. Given a sequence $\left(\mu_{n}\right)_{n \in \mathbb{N}}$ of measures in $\mathcal{M}^{+}$converging to some measure $\mu \in \mathcal{M}^{+}$in the vague topology $\widetilde{\sigma}$, for every $s, t \in \mathbb{R}$, with $s \leq t$, one has

(a) $\mu([s, t]) \geq \lim \sup _{n \rightarrow \infty} \mu_{n}([s, t])$, and

(b) $\mu([s, t]) \leq \liminf _{n \rightarrow \infty} \mu_{n}([s-\delta, t+\delta])$ for every $\delta>0$.

We are now ready to give a characterization of the $L_{l o c}^{1}$-equicontinuity for subsets of positive functions in $L_{l o c}^{1}$.

Theorem 2.23. Let $S \subset L_{\text {loc }}^{1}$ be a set of positive functions and let $M \subset \mathcal{M}_{a c}^{+}$be the set of absolutely continuous measures whose densities are the functions of $S$. Then, the following statements are equivalent.

(i) $S$ is $L_{l o c}^{1}$-equicontinuous.

(ii) $M$ is relatively compact in $\left(\mathcal{M}^{+}, \widetilde{\sigma}\right)$ and $\operatorname{cls}_{\left(\mathcal{M}^{+}, \widetilde{\sigma}\right)}(M) \subset \mathcal{M}_{a c}^{+} \oplus \mathcal{M}_{s c}^{+}$.

Proof. (i) $\Rightarrow$ (ii). One immediately has that $M$ is relatively compact thanks to Remark 2.18 and Remark 2.21. Let us prove that $\operatorname{cls}_{\left(\mathcal{M}^{+}, \widetilde{\sigma}\right)}(M) \subset \mathcal{M}_{a c}^{+} \oplus \mathcal{M}_{s c}^{+}$. Let $\mu$ be a measure in $\operatorname{cls}_{\left(\mathcal{M}^{+}, \widetilde{\sigma}\right)}(M)$ and $\left(\mu_{n}\right)_{n \in \mathbb{N}}$ be a sequence in $M$ converging to $\mu$. We have that fixed $\varepsilon>0$ there exists a $\delta>0$ such that, due to (i), for every $n \in \mathbb{N}$ we have

$$
\mu_{n}([s, t])<\varepsilon \quad \text { whenever } t-s<\delta .
$$

Now consider $-r \leq s \leq t \leq r$ and $t-s<\delta / 2$. Then, from Proposition 2.22(b), one has that

$$
\mu([s, t]) \leq \liminf _{n \rightarrow \infty} \mu_{n}([s-\delta / 4, t+\delta / 4]) \leq \varepsilon,
$$

which means that $\mu$ has a continuous variation on $t$ and $s$. Therefore, it can not have a purely discontinuous component which means that $\mu \in \mathcal{M}_{a c}^{+} \oplus \mathcal{M}_{s c}^{+}$.

(ii) $\Rightarrow$ (i). Assume on the contrary that $S$ is not $L_{l o c}^{1}$-equicontinuous. Therefore, there exist $r>0$ and $\varepsilon>0$ such that for any $n \in \mathbb{N}$ there is a function $m_{n}(\cdot) \in S$ and an interval $\left[s_{n}, t_{n}\right] \subset[-r, r]$ with $t_{n}-s_{n}<1 / n$, such that

$$
\int_{s_{n}}^{t_{n}} m_{n}(u) d u>\varepsilon
$$

Firstly notice that, since the sequences $\left(s_{n}\right)_{n \in \mathbb{N}}$ and $\left(t_{n}\right)_{n \in \mathbb{N}}$ take values in the compact set $[-r, r]$ and $t_{n}-s_{n} \rightarrow 0$ as $n \rightarrow \infty$, then there exists $\tau \in[-r, r]$ such 
that, up to a subsequence $s_{n} \rightarrow \tau$ and $t_{n} \rightarrow \tau$ as $n \rightarrow \infty$. Now, let us consider the sequence $\left(\mu_{n}\right)_{n \in \mathbb{N}}$ of absolutely continuous measure in $M$ such that for any $n \in \mathbb{N}$ the function $m_{n}(\cdot)$ is the density of the measure $\mu_{n}$. By (ii), $\left(\mu_{n}\right)_{n \in \mathbb{N}}$ vaguely converges, up to a subsequence, to a measure $\mu \in \mathcal{M}_{a c}^{+} \oplus \mathcal{M}_{s c}^{+}$. Then, for the given $\varepsilon$, there exists a $\delta>0$ such that

$$
\mu([\tau-\delta, \tau+\delta])<\varepsilon
$$

Moreover, there exists $n_{0} \in \mathbb{N}$ such that $\left[s_{n}, t_{n}\right] \subset[\tau-\delta, \tau+\delta]$ for all $n>n_{0}$. However, from Proposition 2.22(a) we have that

$$
\mu([\tau-\delta, \tau+\delta]) \geq \limsup _{n \rightarrow \infty} \mu_{n}([\tau-\delta, \tau+\delta]) \geq \limsup _{n \rightarrow \infty} \mu_{n}\left(\left[s_{n}, t_{n}\right]\right)>\varepsilon,
$$

which contradicts (2.13). Therefore, we deduce that $S$ is $L_{l o c}^{1}$-equicontinuous, which concludes the proof.

At follows, we extend the notions of $L_{l o c}^{p}$-boundedness and $L_{l o c}^{1}$-equicontinuity given in Definitions 2.16 and 2.17, respectively, to sets of Carathéodory functions through their $m$-bounds and/or $l$-bounds. A special attention will be paid to the set of the time translations of a function, because it plays a key role in the construction of its hull (see Definition 1.10), and consequently in the continuity of a skew-product flow induced by a non-autonomous ordinary differential equation.

Definition 2.24 ( $L_{l o c}^{1}$-boundedness and $L_{l o c}^{1}$-equicontinuity for Carathéodory functions). Consider $1 \leq p<\infty$. We say that

(i) a set $E \subset \mathfrak{C}_{p}\left(\mathbb{R}^{M}\right)$ has $L_{\text {loc-bounded (resp. L Loc }}^{p}$-equicontinuous) m-bounds, if for any $j \in \mathbb{N}$ there exists a set $S^{j} \subset L_{l o c}^{p}$ of $m$-bounds of the functions of $E$ on $B_{j}$, such that $S^{j}$ is $L_{l o c}^{p}$-bounded (resp. $L_{l o c}^{1}$-equicontinuous);

(ii) a function $f \in \mathfrak{C}_{p}\left(\mathbb{R}^{M}\right)$ has $L_{\text {loc }}^{p}$-bounded (resp. $L_{\text {loc }}^{1}$-equicontinuous) $m$ bounds if the set $\left\{f_{t} \mid t \in \mathbb{R}\right\}$ has $L_{l o c}^{p}$-bounded (resp. $L_{l o c}^{1}$-equicontinuous) $m$-bounds;

(iii) a set $E \subset \mathfrak{L C}_{p}\left(\mathbb{R}^{M}\right)$ has $L_{\text {loc }}^{p}$-bounded (resp. L L loc-equicontinuous) l-bounds, if for any $j \in \mathbb{N}$, the set $S^{j} \subset L_{l o c}^{p}$, made up of the optimal l-bounds on $B_{j}$ of the functions in $E$, is $L_{l o c}^{p}$-bounded (resp. $L_{l o c}^{1}$-equicontinuous);

(iv) a function $f \in \mathfrak{L C}_{p}\left(\mathbb{R}^{M}\right)$ has $L_{\text {loc }}^{p}$-bounded (resp. $L_{\text {loc }}^{1}$-equicontinuous) lbounds if the set $\left\{f_{t} \mid t \in \mathbb{R}\right\}$ has $L_{l o c}^{p}$-bounded (resp. $L_{l o c}^{1}$-equicontinuous) l-bounds.

Remark 2.25. Notice that (ii) and (iv) in Definition 2.24 are well-posed. In fact, if $f \in \mathfrak{C}_{p}\left(\mathbb{R}^{M}\right)$, then for any $t \in \mathbb{R}$ one has that $f_{t}(\cdot, \cdot)=f(t+\cdot, \cdot)$ is Borelmeasurable and, for any $j \in \mathbb{N}$, if $x \in B_{j} \subset \mathbb{R}^{N}$, then for almost every $s \in \mathbb{R}$ one has

$$
\left|f_{t}(s, x)\right|=|f(t+s, x)| \leq m^{j}(t+s)=m_{t}^{j}(s)
$$


where $m^{j}(\cdot) \in L_{l o c}^{p}$ is an $m$-bound for $f$ on $B_{j}$. As a consequence for any $j \in \mathbb{N}$, the function $f_{t}$ has an $m$-bound on $B_{j}$. Therefore, $f_{t}$ satisfies $(\mathrm{C} 1)$ and $(\mathrm{C} 2)$ and thus $f_{t} \in \mathfrak{C}_{p}\left(\mathbb{R}^{M}\right)$. Furthermore, if $f \in \mathfrak{L C}_{p}\left(\mathbb{R}^{M}\right)$, one has that, for any $j \in \mathbb{N}$, if $x, y \in B_{j} \subset \mathbb{R}^{N}$, then for almost every $s \in \mathbb{R}$ one has

$$
\left|f_{t}(s, x)-f_{t}(s, y)\right|=\left|f(s+t, x)-f_{t}(s+t, y)\right| \leq l^{j}(t+s)|x-y|=l_{t}^{j}(s)|x-y|,
$$

where $l^{j}(\cdot) \in L_{l o c}^{p}$ is the optimal $l$-bound for $f$ on $B_{j}$. As a consequence for any $j \in \mathbb{N}$, the function $f_{t}$ has an $l$-bound on $B_{j}$ and thus, it also satisfies $(\mathbf{L})$ in Definition 2.1 and thus $f_{t} \in \mathfrak{L} \mathfrak{C}_{p}\left(\mathbb{R}^{M}\right)$.

\subsection{Topological closure}

As a first application of the notions introduced in Definition 2.24, we aim to investigate if such properties can be extended to the limit functions once we take the closure in a suitable topological space. The possibility of maintaining properties on the $m$-bounds and/or on the $l$-bounds through the limits is actually crucial because it allows to treat the closure of sets as "homogeneous" (with respect to a specific property), as we will see in the following sections. Just to cite two essential applications, for a converging sequence in a set with $L_{l o c}^{p}$ bounded $l$-bounds we will be able to deduce the existence and uniqueness of solutions for the induced limit differential problem or that, within a closed set with $L_{l o c}^{1}$-equicontinuous $m$-bounds, all the solutions of the respective differential problems share the same moduli of continuity.

The following three cases are of interest for the rest of our work.

(a) The closure of any subset of $\mathfrak{S} \mathfrak{C}$ or $\mathfrak{L} \mathfrak{C}$ with respect to any of the introduced topologies.

(b) The closure of any subset of $\mathfrak{S C}_{p}$ or $\mathfrak{L C}_{p}$, with $p \geq 1$, with respect to any of the introduced strong topologies.

(c) The closure of any subset of $\Theta \mathfrak{C}$ or $\mathfrak{W} \Theta \mathfrak{C}$ respectively endowed with the topologies $\mathcal{T}_{\Theta}$ and $\sigma_{\Theta}$.

Notice that in case (b), only the subcase $p=1$ lays within the scope of (a). Furthermore, case (c) does not fall within any of the other two cases because the closure is taken with respect to either, the strong topology $\mathcal{T}_{\Theta}$ or the weak topology $\sigma_{\Theta}$, but in larger spaces than in case (a).

Proposition 2.26. Let $\mathcal{T}$ be any of the topologies introduced in Section 2.2.

(i) If $E \subset \mathfrak{S C}\left(\mathbb{R}^{M}\right)$ (resp. $E \subset \mathfrak{L} \mathfrak{C}\left(\mathbb{R}^{M}\right)$ ) admits $L_{\text {loc }}^{1}$-bounded $m$-bounds (resp. $\quad L_{\text {loc }}^{1}$-bounded l-bounds) then $\operatorname{cls}_{\left(\mathfrak{S} \mathfrak{C}\left(\mathbb{R}^{M}\right), \mathcal{T}\right)}(E)$ has $L_{\text {loc }}^{1}$-bounded $m$ bounds (resp. $\operatorname{cls}_{\left(\mathfrak{S C}\left(\mathbb{R}^{M}\right), \mathcal{T}\right)}(E) \subset \mathfrak{L} \mathfrak{C}\left(\mathbb{R}^{M}\right)$ and it has $L_{\text {loc }}^{1}$-bounded l-bounds). 
(ii) If $E \subset \mathfrak{S C}\left(\mathbb{R}^{M}\right)$ (resp. $E \subset \mathfrak{L}\left(\mathbb{R}^{M}\right)$ ) admits $L_{\text {loc }}^{1}$-equicontinuous $m$ bounds (resp. $L_{\text {loc }}^{1}$-equicontinuous l-bounds), then $\operatorname{cls}_{\left(\mathfrak{S c}_{p}\left(\mathbb{R}^{M}\right), \mathcal{T}\right)}(E)$ has $L_{\text {loc }}^{1}$ equicontinuous $m$-bounds $\left(\right.$ resp. $\operatorname{cls}_{\left(\mathfrak{S C}\left(\mathbb{R}^{M}\right), \mathcal{T}\right)}(E) \subset \mathfrak{L} \mathfrak{C}\left(\mathbb{R}^{M}\right)$ and it has $L_{\text {loc }}^{1}$ equicontinuous l-bounds).

(iii) If $f \in \mathfrak{S C}\left(\mathbb{R}^{M}\right)$ (resp. $f \in \mathfrak{L} \mathfrak{C}\left(\mathbb{R}^{M}\right)$ ) admits $L_{\text {loc }}^{1}$-bounded $m$-bounds (resp. $L_{\text {loc }}^{1}$-bounded l-bounds) then any $g \in \operatorname{Hull}_{\left(\mathfrak{S C}\left(\mathbb{R}^{M}\right), \mathcal{T}\right)}(f)$ has $L_{\text {loc }}^{1}$-bounded m-bounds (resp. $L_{l o c}^{1}$-bounded l-bounds).

(iv) If $f \in \mathfrak{S C}\left(\mathbb{R}^{M}\right)$ (resp. $f \in \mathfrak{L} \mathfrak{C}\left(\mathbb{R}^{M}\right)$ ) admits $L_{\text {loc }}^{1}$-equicontinuous $m$-bounds (resp. $L_{\text {loc }}^{1}$-equicontinuous l-bounds), then any $g \in \operatorname{Hull}_{\left(\mathfrak{S C}\left(\mathbb{R}^{M}\right), \mathcal{T}\right)}(f)$ has $L_{\text {loc }}^{1}$-equicontinuous m-bounds (resp. $L_{\text {loc }}^{1}$-equicontinuous l-bounds).

Proof. By (2.10), if we prove the result for the topology $\sigma_{D}$, we have it for all the other topologies.

(i). Let us firstly reason for the $m$-bounds and, in order to simplify the notation, let $\bar{E}$ denote the set $\operatorname{cls}_{\left(\mathfrak{S C}\left(\mathbb{R}^{M}\right), \sigma_{D}\right)}(E)$. As we are applying the topological closure in $\left(\mathfrak{S C}\left(\mathbb{R}^{M}\right), \sigma_{D}\right)$, we already know that each function in $\bar{E}$ admits an optimal $m$-bound. However, without any additional information, it is difficult to say whether the optimal $m$-bounds of the limit functions allow us to preserve the property of $L_{l o c}^{1}$-boundedness in $\bar{E}$ or not. The idea then, is that, for each function $f \in \bar{E} \backslash E$, we consider a sequence $\left(f_{n}\right)_{n \in \mathbb{N}}$ in $E$ converging to $f$ with respect to $\sigma_{D}$, and we build an $m$-bound for $f$ starting from the optimal $m$-bounds of $\left(f_{n}\right)_{n \in \mathbb{N}}$. In such a way, the obtained $m$-bound encloses some additional information which allows us to prove $L_{l o c}^{1}$-boundedness for $\bar{E}$. Notice that, for any function $f$ and $j \in \mathbb{N}$, if this kind of property is true for a generic $m$-bound for $f$ on $B_{j}$, then it is also true for the optimal $m$-bound for $f$ on $B_{j}$ (see Definition 2.1).

Fix $j \in \mathbb{N}$ and, for any $n \in \mathbb{N}$, let $m_{n}^{j}$ be the optimal $m$-bound for $f_{n}$ on $B_{j}$ and $\mu_{n}^{j} \in \mathcal{M}^{+}$be the positive absolutely continuous measure (with respect to the Lebesgue measure) with density $m_{n}^{j}(\cdot)$. By hypothesis, the set $\left\{m_{n}^{j}(\cdot) \mid\right.$ $n \in \mathbb{N}\}$ is $L_{l o c}^{1}$-bounded. Hence, due to Remark 2.21, the sequence of induced measures $\left(\mu_{n}^{j}\right)_{n \in \mathbb{N}}$, is relatively compact in $\left(\mathcal{M}^{+}, \widetilde{\sigma}\right)$ and thus vaguely converges, up to a subsequence, to a measure $\mu^{j} \in \mathcal{M}^{+}$. Moreover, by Lebesgue-Besicovitch differentiation theorem (see Ambrosio et al. [1, Theorem 2.22, p.54]), there exists $m^{j}(\cdot) \in L_{l o c}^{1}$ such that

$$
m^{j}(t)=\lim _{h \rightarrow 0} \frac{\mu^{j}([t, t+h])}{h}, \quad \text { for a.e. } t \in \mathbb{R}
$$

and $m^{j}(\cdot)$ is the density of the absolutely continuous part of the Radon-Nikodým decomposition of $\mu^{j}$ in each compact interval. We claim that $m^{j}(\cdot)$ is an $m$-bound for $f$ on $B_{j}$. Let us firstly fix $x \in D \cap B_{j}$, and take $t, h \in \mathbb{Q}$, with $h>0$, and 
$\phi \in C_{C}^{+}(\mathbb{R})$ such that $\phi \equiv 1$ in $[t, t+h]$. Then, we have

$$
\begin{aligned}
\left|\frac{1}{h} \int_{t}^{t+h} f(s, x) d s\right| & =\lim _{n \rightarrow \infty}\left|\frac{1}{h} \int_{t}^{t+h} f_{n}(s, x) d s\right| \leq \lim _{n \rightarrow \infty} \frac{1}{h} \int_{t}^{t+h} m_{n}^{j}(s) d s \\
& \leq \lim _{n \rightarrow \infty} \frac{1}{h} \int_{\mathbb{R}} \phi(s) d \mu_{n}^{j}(s)=\frac{1}{h} \int_{\mathbb{R}} \phi(s) d \mu^{j}(s) .
\end{aligned}
$$

Moreover, thanks to the regularity of $\mu^{j}$, one has

$$
\mu^{j}([t, t+h])=\inf \left\{\int_{\mathbb{R}} \phi(s) d \mu^{j}(s) \mid \phi \in C_{C}^{+}(\mathbb{R}), \phi \equiv 1 \text { in }[t, t+h]\right\} .
$$

Therefore, gathering the previous two formulas, we obtain

$$
\left|\frac{1}{h} \int_{t}^{t+h} f(s, x) d s\right| \leq \frac{\mu^{j}([t, t+h])}{h} .
$$

Now, consider $t, h \in \mathbb{R}$, with $h>0$, and let $\left(s_{n}\right)_{n \in \mathbb{N}}$ and $\left(t_{n}\right)_{n \in \mathbb{N}}$ be two sequences in $\mathbb{Q}$ such that, as $n \rightarrow \infty, s_{n} \downarrow t$ and $t_{n} \uparrow t+h$, respectively. By (2.16), applied on the intervals $\left[s_{n}, t_{n}\right]$, and noticing that $\mu^{j}\left(\left[s_{n}, t_{n}\right)\right] \leq \mu^{j}([t, t+h])$ for every $n \in \mathbb{N}$, one can write

$$
\left|\frac{1}{h} \int_{s_{n}}^{t_{n}} f(s, x) d s\right| \leq \frac{\mu^{j}([t, t+h])}{h}, \quad \text { for all } n \in \mathbb{N} .
$$

Hence, passing to the limit as $n \rightarrow \infty$ and using the continuity of the integral, one obtains (2.16) for every $t, h \in \mathbb{R}$ with $h>0$. Now, as $h \rightarrow 0$ (see [24, Corollary III.12.7, p.216]) and using (2.15), we obtain that for almost every $t \in \mathbb{R}$,

$$
|f(t, x)| \leq m^{j}(t),
$$

for the fixed $x \in D \cap B_{j}$. For every fixed $x \in D \cap B_{j}$ let us now denote by $R(x)$ the subset of $\mathbb{R}$ such that meas $\mathbb{R}(\mathbb{R} \backslash R(x))=0$ and (2.18) holds for all $t \in R(x)$. Such a set clearly depends on $x \in D \cap B_{j}$. However, since $D$ is numerable, by simply intersecting all the possible $R(x)$, with $x \in D \cap B_{j}$, (there is only a numerable quantity of them), one can obtain a set $R_{0} \subset \mathbb{R}$ of full measure for which (2.18) holds for any $x \in D \cap B_{j}$. Finally, by the continuity of $f(t, \cdot)$, we obtain the result for almost every $t \in \mathbb{R}$ for all $x \in B_{j}$, and $m^{j}$ provides an $m$-bound for $f$ in $B_{j}$, as claimed.

Now, we prove that $\bar{E}$ admits $L_{l o c}^{1}$-bounded $m$-bounds. For each $f \in \bar{E}$ and any $j \in \mathbb{N}$, let $m_{f}^{j}$ be either, the optimal $m$-bound of $f$ on $B^{j}$ if $f \in E$, or the $m$-bound given by (2.15), i.e. the absolutely continuous part of a limit measure, if $f \in \bar{E} \backslash E$. Moreover, for each $f \in \bar{E}$, let $\left(f_{n}\right)_{n \in \mathbb{N}}$ be a sequence in $E$ converging to $f$ with respect to $\sigma_{D}$. Consider $j \in \mathbb{N}, r>0$ and $\phi \in C_{C}^{+}$such that $\operatorname{supp} \phi \subset[-r-1, r+1]$ and $\phi \equiv 1$ in $[-r, r]$, then, we have

$$
\begin{aligned}
\int_{-r}^{r} m_{f}^{j}(t) d t & \leq \int_{\mathbb{R}} \phi(t) m_{f}^{j}(t) d t \leq \lim _{n \rightarrow \infty} \int_{\mathbb{R}} \phi(t) m_{f_{n}}^{j}(t) d t \\
& \leq \sup _{g \in E} \int_{-r-1}^{r+1} m_{g}^{j}(t) d t<\infty,
\end{aligned}
$$


where the last inequality comes from the assumption of $L_{l o c}^{1}$-boundedness for the $m$-bounds of $E$. Hence, taking the superior on $f \in \bar{E}$ in the previous chain of inequalities, we obtain

$$
\sup _{f \in \bar{E}} \int_{-r}^{r} m_{f}^{j}(t) d t<\infty .
$$

Therefore, $\bar{E}$ admits $L_{l o c}^{1}$-bounded $m$-bounds, which concludes this part of the proof.

Now, consider $E \subset \mathfrak{L} \mathfrak{C}\left(\mathbb{R}^{M}\right)$ with $L_{\text {loc }}^{1}$-bounded $l$-bounds. Reasoning as in the first part of the proof, we have that the sequence of absolutely continuous measures with densities $\left(l_{n}^{j}(t)\right)_{n \in \mathbb{N}}$ vaguely converges, up to a subsequence, to a positive measure whose absolutely continuous part has density $l^{j}(\cdot) \in L_{l o c}^{1}$. Additionally, for any $x, y \in D \cap B_{j}$ with $x \neq y$ the following inequality holds

$$
|f(t, x)-f(t, y)| \leq l^{j}(t)|x-y| \quad \text { for a.e. } t \in \mathbb{R} .
$$

Notice that the set $R(x, y) \subset \mathbb{R}$ of total measure for which (2.20) holds, depends on $x$ and $y$. However, since $D$ is numerable, again, by intersecting all the possible $R(x, y)$ (there is only a numerable quantity of them), one can obtain a set $R_{0} \subset \mathbb{R}$ of full measure for which (2.20) holds for any $x, y \in D \cap B_{j}$. An extension of the previous inequality to the entire $B_{j}$ is thus achieved thanks to the continuity of $f$ with respect to the variable $x$. Therefore, $f \in \mathfrak{L C}_{p}\left(\mathbb{R}^{M}\right)$ and thus $\bar{E} \subset \mathfrak{L C}\left(\mathbb{R}^{M}\right)$. Finally, reasoning exactly as in (2.19), one obtains that $\bar{E}$ has $L_{l o c}^{1}$-bounded $l$ bounds.

(ii). Again, let us firstly work on the $m$-bounds. For each $f \in \bar{E}$ and any $j \in \mathbb{N}$ let $m_{f}^{j}$ be either, the optimal $m$-bound of $f$ on $B^{j}$ if $f \in E$, or the $m$ bound given by (2.15) if $f \in \bar{E} \backslash E$, i.e. the absolutely continuous part of a limit measure. Moreover, for each $f \in \bar{E}$ let $\left(f_{n}\right)_{n \in \mathbb{N}}$ be a sequence in $E$ converging to $f$ with respect to $\sigma_{D}$. By the $L_{l o c}^{1}$-equicontinuity of the $m$-bounds, we have that for each $j \in \mathbb{N}$, and $r, \varepsilon>0$ there exists $\delta=\delta(r, \varepsilon)>0$ such that

$$
\text { for all } t, s \in[-r, r]: \quad 0<t-s<\delta \Rightarrow \sup _{f \in E} \int_{s}^{t} m_{f}^{j}(u) d u<\varepsilon \text {. }
$$

Moreover, consider $t, s \in[-r, r]$ with $s<t$ and $t-s<\delta / 3$ and $\phi \in C_{C}^{+}$such that $\operatorname{supp} \phi \subset[s-\delta / 3, t+\delta / 3]$ and $\phi \equiv 1$ in $[s, t]$. Then, we have

$$
\begin{aligned}
\int_{s}^{t} m_{f}^{j}(u) d u & \leq \int_{\mathbb{R}} \phi(u) m_{f}^{j}(u) d u \leq \lim _{n \rightarrow \infty} \int_{\mathbb{R}} \phi(u) m_{f_{n}}^{j}(u) d u \\
& \leq \sup _{g \in E} \int_{s-\delta / 3}^{t+\delta / 3} m_{g}^{j}(u) d u<\varepsilon .
\end{aligned}
$$

Hence, taking the superior on $f \in \bar{E}$ in the previous chain of inequalities, we obtain

$$
\sup _{f \in \bar{E}} \int_{s}^{t} m_{f}^{j}(u) d u<\varepsilon
$$


Therefore, $\bar{E}$ admits $L_{l o c}^{1}$-equicontinuous $m$-bounds. The result for the $l$-bounds can be obtained by analogous reasoning.

(iii) and (iv) are a direct consequence of (i) and (ii), respectively.

Proposition 2.27. Let $\mathcal{T}$ be any of the strong topologies introduced in Section 2.2 and $1 \leq p<\infty$.

(i) If $E \subset \mathfrak{S C}_{p}\left(\mathbb{R}^{M}\right)$ (resp. $E \subset \mathfrak{L C}_{p}\left(\mathbb{R}^{M}\right)$ ) admits $L_{\text {loc }}^{p}$-bounded m-bounds (resp. $L_{\text {loc }}^{p}$-bounded l-bounds) then $\operatorname{cls}_{\left(\mathfrak{S C}_{p}\left(\mathbb{R}^{M}\right), \mathcal{T}\right)}(E)$ has $L_{\text {loc }}^{p}$-bounded $m$ bounds (resp. $\operatorname{cls}_{\left(\mathfrak{S C}\left(\mathbb{R}^{M}\right), \mathcal{T}\right)}(E) \subset \mathfrak{L} \mathfrak{C}\left(\mathbb{R}^{M}\right)$ and it has $L_{\text {loc }}^{p}$-bounded l-bounds).

(ii) If $E \subset \mathfrak{S C}_{p}\left(\mathbb{R}^{M}\right)$ (resp. $E \subset \mathfrak{L C}_{p}\left(\mathbb{R}^{M}\right)$ ) admits $L_{\text {loc }}^{1}$-equicontinuous $m$ bounds (resp. $L_{\text {loc }}^{1}$-equicontinuous l-bounds), then $\operatorname{cls}_{\left(\mathfrak{S C}_{p}\left(\mathbb{R}^{M}\right), \mathcal{T}\right)}(E)$ has $L_{\text {loc }}^{1}$ equicontinuous m-bounds (resp. $\operatorname{cls}_{\left(\mathfrak{S} \mathfrak{C}\left(\mathbb{R}^{M}\right), \mathcal{T}\right)}(E) \subset \mathfrak{L} \mathfrak{C}\left(\mathbb{R}^{M}\right)$ and it has $L_{\text {loc }}^{1}$ equicontinuous l-bounds).

(iii) If $f \in \mathfrak{S C}_{p}\left(\mathbb{R}^{M}\right)$ (resp. $f \in \mathfrak{L C}_{p}\left(\mathbb{R}^{M}\right)$ ) has $L_{\text {loc }}^{p}$-bounded m-bounds (resp. $L_{\text {loc }}^{p}$-bounded l-bounds) then any $g \in \operatorname{Hull}_{\left(\mathfrak{S C}_{p}\left(\mathbb{R}^{M}\right), \mathcal{T}\right)}(f)$ has $L_{l o c}^{p}$-bounded m-bounds (resp. L Loc-bounded l-bounds).

(iv) If $f \in \mathfrak{S C}_{p}\left(\mathbb{R}^{M}\right)$ (resp. $f \in \mathfrak{L C}_{p}\left(\mathbb{R}^{M}\right)$ ) has $L_{\text {loc }}^{1}$-equicontinuous $m$-bounds (resp. $L_{\text {loc }}^{1}$-equicontinuous l-bounds), then any $g \in \operatorname{Hull}_{\left(\mathfrak{S C}_{p}\left(\mathbb{R}^{M}\right), \mathcal{T}\right)}(f)$ has $L_{l o c}^{1}$-equicontinuous m-bounds (resp. $L_{l o c}^{1}$-equicontinuous l-bounds).

Proof. For all the four statements (i) to (iv), the case $p=1$ is included in Proposition 2.26. Now, consider $p>1$ and let us work for the case of the $m$-bounds in (i). In particular we can apply the same reasoning used in the proof of Proposition 2.26 recalling that $L_{l o c}^{p} \subset L_{l o c}^{1}$, and we only need to prove that the function $m^{j}(\cdot) \in L_{l o c}^{1}$, provided by (2.15), is also in $L_{l o c}^{p}$. By hypothesis $\left\{m_{n}^{j}(\cdot) \mid n \in \mathbb{N}\right\}$ is $L_{l o c}^{p}$-bounded and, by Alaoglu-Bourbaki theorem, for every $r>0$ the closed balls of $L^{p}([-r, r])$ are relatively compact in the weak topology $\sigma\left(L^{p}([-r, r]), L^{q}([-r, r])\right)$. Therefore, if $\left(m_{i_{n}}^{j}(\cdot)\right)_{n \in \mathbb{N}}$ is a weakly convergent subsequence of $\left(m_{n}^{j}(\cdot)\right)_{n \in \mathbb{N}}$ with limit $m^{*}(\cdot) \in L^{p}([-r, r])$, then the sequence of induced measures $\left(\mu_{i_{n}}^{j}\right)_{n \in \mathbb{N}}$ vaguely converges to the absolutely continuous measure whose density is $m^{*}(\cdot)$ in $[-r, r]$. Hence, since Equation $(2.15)$ holds, $m^{*}(\cdot)$ has to coincide with $m^{j}(\cdot)$ in $[-r, r]$.

The same reasoning applies to the $l$-bounds and to the rest of the cases in (ii), whereas (iii) and (iv) are a direct consequence of (i) and (ii), respectively.

The resulted presented so far, involve the spaces $\mathfrak{S C}_{p}\left(\mathbb{R}^{M}\right)$ and $\mathfrak{L C}_{p}\left(\mathbb{R}^{M}\right)$. At this point we would like to address the analogous problem in the space $\left(\mathfrak{W} \Theta \mathfrak{C}\left(\mathbb{R}^{M}\right), \sigma_{\Theta}\right)$. In other words, we aim to show that the existence of $L_{l o c^{-}}^{1}$ bounded or $L_{l o c}^{1}$-equicontinuous $m$-bounds (respectively $l$-bounds) for a set $E \subset$ $\mathfrak{W} \Theta \mathfrak{C}\left(\mathbb{R}^{M}\right)$ (resp. $\left.E \subset \mathfrak{L} \mathfrak{C}\left(\mathbb{R}^{M}\right)\right)$ is inherited by all the elements of the closure 
of $E$ with respect to $\sigma_{\Theta}$ (see Definition 2.4). A special case is the one in which $E$ is the set of the time translations of a function and, there, a problem arises. As shown in Remark 2.25, if a function $f$ belongs to either $\mathfrak{S C}_{p}\left(\mathbb{R}^{M}\right), \mathfrak{L} \mathfrak{C}_{p}\left(\mathbb{R}^{M}\right)$ or $\Theta \mathfrak{C}_{p}\left(\mathbb{R}^{M}\right)$, then any time-translation of $f$ (see Definition 1.9) also belongs to the same set. Nevertheless, the same result is not so obvious for $\mathfrak{W} \Theta \mathfrak{C}\left(\mathbb{R}^{M}\right)$ and thus, before proceeding, we include a proof of this fact.

Lemma 2.28. Let $\Theta$ be a suitable set of moduli of continuity as in Definition 2.3. If $t \in \mathbb{R}$ and $f \in \mathfrak{W} \Theta \mathfrak{C}\left(\mathbb{R}^{M}\right)$, then $f_{t} \in \mathfrak{W} \Theta \mathfrak{C}\left(\mathbb{R}^{M}\right)$.

Proof. Notice that for any fixed $f \in \mathfrak{W} \Theta \mathfrak{C}\left(\mathbb{R}^{M}\right)$ and $t \in \mathbb{R}$, the function $f_{t}$ trivially satisfies $(\mathrm{C} 1)$ and $(\mathrm{C} 2)$. In order to prove that condition (W) of Definition 2.4 holds, consider $j \in \mathbb{N}, I=\left[q_{1}, q_{2}\right], q_{1}, q_{2} \in \mathbb{Q}$, and a sequence $\left(x_{n}(\cdot)\right)_{n \in \mathbb{N}}$ in $\mathcal{K}_{j}^{I}$ converging uniformly to $x(\cdot) \in \mathcal{K}_{j}^{I}$. Then, one has that

$$
\begin{aligned}
\lim _{n \rightarrow \infty} \mid \int_{I}\left[f_{t}\right. & \left.\left(s, x_{n}(s)\right)-f_{t}(s, x(s))\right] d s \mid \\
& =\lim _{n \rightarrow \infty}\left|\int_{I}\left[f\left(s+t, x_{n}(s)\right)-f(s+t, x(s))\right] d s\right| \\
& =\lim _{n \rightarrow \infty}\left|\int_{I+t}\left[f\left(u, x_{n}(u-t)\right)-f(u, x(u-t))\right] d u\right| .
\end{aligned}
$$

Considering an interval $J$ with rational extrema such that $I \cup(I+t) \subset J$ and, up to an extension by constants to $J$, the functions $x_{n}(\cdot-t)$ and $x(\cdot-t)$ are in $\mathcal{K}_{j}^{J}$. If $t \in \mathbb{Q}$ we immediately obtain the thesis thanks to Lemma 2.14(i). If $t \in \mathbb{R} \backslash \mathbb{Q}$, fix $\varepsilon>0$ and let $\delta_{1}, \delta_{2}>0$ be such that

$$
\int_{q_{1}+t-\delta_{1}}^{q_{1}+t} m_{f}^{j}(u) d u<\frac{\varepsilon}{4} \quad \text { and } \quad \int_{q_{2}+t}^{q_{2}+t+\delta_{2}} m_{f}^{j}(u) d u<\frac{\varepsilon}{4},
$$

where $m_{f}^{j}(\cdot)$ is the $m$-bound of $f$ on $B_{j}$. The previous inequalities hold because of the continuity of the integral. Thus, denoted by $\delta=\min \left\{\delta_{1}, \delta_{2}\right\}$, consider $p_{1} \in\left[q_{1}+t-\delta, q_{1}+t\right] \cap \mathbb{Q}$ and $p_{2} \in\left[q_{2}+t, q_{2}+t+\delta\right] \cap \mathbb{Q}$. Starting from the last step of the chain of equalities in (2.21), one has

$$
\begin{aligned}
\lim _{n \rightarrow \infty}\left|\int_{q_{1}+t}^{q_{2}+t}\left[f\left(u, x_{n}(u-t)\right)-f(u, x(u-t))\right] d u\right| \\
\leq \lim _{n \rightarrow \infty}\left|\int_{p_{1}}^{p_{2}}\left[f\left(u, x_{n}(u-t)\right)-f(u, x(u-t))\right] d u\right| \\
\quad+2 \int_{p_{1}}^{q_{1}+t} m_{f}^{j}(u) d u+2 \int_{q_{2}+t}^{p_{2}} m_{f}^{j}(u) d u \\
\leq \lim _{n \rightarrow \infty}\left|\int_{p_{1}}^{p_{2}}\left[f\left(u, x_{n}(u-t)\right)-f(u, x(u-t))\right] d u\right|+\varepsilon .
\end{aligned}
$$

Therefore, we obtain the thesis, thanks to Lemma 2.14(i), putting together the previous chain of inequalities and (2.21). 
Thanks to the previous lemma, now we can safely proceed to the following theorem, whose proof, however, contains an additional difficulty compared to the ones of Theorems 2.26 and 2.27, due to the fact that the functions in $\mathfrak{W} \Theta \mathfrak{C}\left(\mathbb{R}^{M}\right)$ are possibly discontinuous in the variable $x$. In fact, recall that the idea of the proofs of Theorems 2.26 and 2.27 is to construct an $m$-bound or an $l$-bound (satisfying the required property in any of the cases) on $\mathbb{R} \times D$ where $D$ is a dense and countable subset of $\mathbb{R}^{N}$, and then extend it to the whole $\mathbb{R}^{N}$ through the continuity in the variable $x$. Clearly, such argument will not work in $\mathfrak{W} \Theta \mathfrak{C}\left(\mathbb{R}^{M}\right)$. The idea, then, is to change the limit function for a new one which coincides with the first one almost everywhere, satisfies the required property on the $m$-bounds or $l$-bounds on $\mathbb{R} \times \mathbb{R}^{N}$ and it is still in $\mathfrak{W} \Theta \mathfrak{C}\left(\mathbb{R}^{M}\right)$.

Proposition 2.29. Let $\Theta$ be a suitable set of moduli of continuity as in Definition 2.3 and $\sigma_{\Theta}$ the topology defined in Definition 2.4.

(i) If $E \subset \mathfrak{W} \Theta \mathfrak{C}\left(\mathbb{R}^{M}\right)$ (resp. $E \subset \mathfrak{L} \mathfrak{C}\left(\mathbb{R}^{M}\right)$ ) admits $L_{\text {loc }}^{1}$-equicontinuous $m$ bounds (resp. $L_{l o c}^{1}$-equicontinuous $l$-bounds), then $\operatorname{cls}_{\left(\mathfrak{w} \Theta \mathfrak{C}\left(\mathbb{R}^{M}\right), \sigma_{\Theta}\right)}(E)$ has $L_{\text {loc }}^{1}$-equicontinuous $m$-bounds $\left(\operatorname{resp} . \operatorname{cls}_{\left(\mathfrak{w} \Theta \mathfrak{C}\left(\mathbb{R}^{M}\right), \sigma_{\Theta}\right)}(E) \subset \mathfrak{L} \mathfrak{C}\left(\mathbb{R}^{M}\right)\right.$ and it has $L_{l o c}^{1}$-equicontinuous $l$-bounds).

(ii) If $E \subset \mathfrak{W} \Theta \mathfrak{C}\left(\mathbb{R}^{M}\right)$ (resp. $E \subset \mathfrak{L} \mathfrak{C}\left(\mathbb{R}^{M}\right)$ ) admits $L_{\text {loc }}^{1}$-bounded $m$-bounds (resp. $\quad L_{l o c}^{1}$-bounded l-bounds) then $\operatorname{cls}_{\left(\mathfrak{w} \Theta \mathfrak{C}\left(\mathbb{R}^{M}\right), \sigma_{\Theta}\right)}(E)$ has $L_{l o c}^{1}$-bounded $m$-bounds (resp. $\operatorname{cls}_{\left(\mathfrak{w} \Theta \mathfrak{C}\left(\mathbb{R}^{M}\right), \sigma_{\Theta}\right)}(E) \subset \mathfrak{L} \mathfrak{C}\left(\mathbb{R}^{M}\right)$ and it has $L_{\text {loc }}^{1}$-bounded l-bounds).

(iii) If $f \in \mathfrak{W} \Theta \mathfrak{C}\left(\mathbb{R}^{M}\right)$ (resp. $f \in \mathfrak{L} \mathfrak{C}\left(\mathbb{R}^{M}\right)$ ) has $L_{\text {loc }}^{1}$-equicontinuous $m$-bounds (resp. $L_{l o c}^{1}$-equicontinuous $l$-bounds), then any $g \in \operatorname{Hull}_{\left(\mathfrak{W} \Theta \mathfrak{C}\left(\mathbb{R}^{M}\right), \sigma_{\Theta}\right)}(f)$ has $L_{l o c}^{1}$-equicontinuous $m$-bounds (resp. $L_{l o c}^{1}$-equicontinuous $l$-bounds).

(iv) If $f \in \mathfrak{W} \Theta \mathfrak{C}\left(\mathbb{R}^{M}\right)$ (resp. $f \in \mathfrak{L} \mathfrak{C}\left(\mathbb{R}^{M}\right)$ ) has $L_{\text {loc }}^{1}$-bounded $m$-bounds (resp. $L_{\text {loc }}^{1}$-bounded $l$-bounds) then any $g \in \operatorname{Hull}_{\left(\mathfrak{W} \Theta \mathfrak{C}\left(\mathbb{R}^{M}\right), \sigma_{\Theta}\right)}(f)$ has $L_{l o c}^{1}$-bounded $m$-bounds (resp. $L_{l o c}^{1}$-bounded $l$-bounds).

Proof. Consider $E \subset \mathfrak{W} \Theta \mathfrak{C}\left(\mathbb{R}^{M}\right)$ with $L_{l o c}^{1}$-equicontinuous $m$-bounds, that is, for every $j \in \mathbb{N}$ there is a family of $m$-bounds for $E$, namely

$$
S^{j}=\left\{m_{f}^{j}(\cdot) \mid f \in E, m_{f}^{j}(\cdot) m \text {-bound for } f \text { on } B_{j}\right\},
$$

satisfying the condition in Definition 2.17. By simplicity and without loss of generality, we will also assume that for every $j \in \mathbb{N}, m_{f}^{j}(t) \leq m_{f}^{j+1}(t)$ for almost every $t \in \mathbb{R}$. Let us denote by $\bar{E}=\operatorname{cls}_{\left(\mathfrak{W} \Theta \mathfrak{C}\left(\mathbb{R}^{M}\right), \sigma_{\Theta}\right)}(E)$, and, for any $f \in \bar{E}$, let $\left(f_{n}\right)_{n \in \mathbb{N}}$ be a sequence in $E$ converging to $f$ in $\left(\mathfrak{W} \Theta \mathfrak{C}\left(\mathbb{R}^{M}\right), \sigma_{\Theta}\right)$.

Fixed $j \in \mathbb{N}$ and reasoning as in the first part of the proof of Proposition 2.26, we can find an $m$-bound for $f$ on $B_{j}$, i.e. a function $m^{j}(\cdot) \in L_{l o c}^{1}$ such that for almost every $t \in \mathbb{R}$ one has that

$$
|f(t, x)| \leq m^{j}(t)
$$


for almost every $x \in B_{j}$. Therefore, we look for a new function $f^{*} \in \mathfrak{W} \Theta \mathfrak{C}\left(\mathbb{R}^{M}\right)$ that coincides with $f$ almost everywhere (in other words, $f$ and $f^{*}$ are in fact representatives of the same element in $\left.\mathfrak{W} \Theta \mathfrak{C}\left(\mathbb{R}^{M}\right)\right)$ and such that for almost every $t \in \mathbb{R}$, the function $f^{*}$ satisfies an inequality of the type (2.22) for all $x \in B_{j}$. Let us consider the function $f^{*}: \mathbb{R} \times \mathbb{R}^{N} \rightarrow \mathbb{R}^{M}$ defined as follows: for every $t \in \mathbb{R}$ we set

$$
f^{*}(t, x)= \begin{cases}f(t, x) & \text { if } x \in B_{i} \backslash B_{i-1} \text { and }|f(t, x)| \leq m^{i+1}(t), i \in \mathbb{N}, \\ 0 & \text { otherwise, }\end{cases}
$$

where 0 represents the null vector of $\mathbb{R}^{N}$. The function $f^{*}$ is Borel measurable and coincides with $f$ almost everywhere. Furthermore, we have that for each $j \in \mathbb{N}$ and for every $t \in \mathbb{R}, f^{*}$ satisfies

$$
\left|f^{*}(t, x)\right| \leq m^{j+1}(t)
$$

for all $x \in B_{j}$. Thus, $f^{*}$ satisfies $(\mathrm{C} 1)$ and $(\mathrm{C} 2)$. Therefore, to prove that $f$ and $f^{*}$ are representatives of the same element in $\mathfrak{W} \Theta \mathfrak{C}\left(\mathbb{R}^{M}\right)$, we only need to prove that $f^{*}$ satisfies $(\mathbf{W})$ in Definition 2.4. In order to do that, we firstly show that for any $I=\left[q_{1}, q_{2}\right], q_{1}, q_{2} \in \mathbb{Q}$, if $x(\cdot) \in \mathcal{K}_{j}^{I}$, then $f^{*}(t, x(t))=f(t, x(t))$ for almost every $t \in I$. Let $x(\cdot) \in \mathcal{K}_{j}^{I}$ and reason locally. Consider $t_{0} \in I$ and assume that $i \leq\left|x\left(t_{0}\right)\right|<i+1$ for some $i \in \mathbb{N}$. Then, by the continuity of $x(\cdot)$, there exist $\delta>0$, such that $|x(t)| \in(i-1, i+1]$ for every $t \in I_{t_{0}}=\left[t_{0}-\delta, t_{0}+\delta\right] \cap I$. Let $\widetilde{x}(\cdot)$ be the continuous function defined on $I$ which coincides with $x(\cdot)$ on $I_{t_{0}}$ and it is its extension by constants on $I \backslash I_{t_{0}}$. Trivially, $\widetilde{x}(\cdot) \in \mathcal{K}_{j}^{I}$ and $\|\widetilde{x}(\cdot)\|_{L^{\infty}(I)} \leq i+1$. Hence, for every $t \in I \cap \mathbb{Q}$ and for every $h \in \mathbb{Q}$, with $h>0$ and $t+h \in I$, considered $\phi \in C_{C}^{+}(\mathbb{R})$ such that $\phi \equiv 1$ in $[t, t+h]$ and using Lemma 2.14, we have that

$$
\begin{aligned}
\left|\frac{1}{h} \int_{t}^{t+h} f(s, \widetilde{x}(s)) d s\right| & =\lim _{n \rightarrow \infty}\left|\frac{1}{h} \int_{t}^{t+h} f_{n}(s, \widetilde{x}(s)) d s\right| \leq \lim _{n \rightarrow \infty} \frac{1}{h} \int_{t}^{t+h} m_{f_{n}}^{i+1}(s) d s \\
& \leq \lim _{n \rightarrow \infty} \frac{1}{h} \int_{\mathbb{R}} \phi(s) m_{f_{n}}^{i+1}(s) d s=\frac{1}{h} \int_{\mathbb{R}} \phi(s) d \mu^{i+1}(s) .
\end{aligned}
$$

Reasoning as in (2.17), one can prove that the previous inequality actually holds for any $t \in I$ and $h>0$ such that $t+h \in I$. Thus, taking the limit as $h \rightarrow 0$, and reasoning as before, we obtain that $|f(t, \widetilde{x}(t))| \leq m^{i+1}(t)$ for almost every $t \in I$. In particular, for almost every $t \in I_{t_{0}}$,

$$
|f(t, x(t))| \leq m^{i+1}(t)
$$

and recalling how $f^{*}$ is defined in (2.23), we have that $f^{*}(t, x(t))=f(t, x(t))$ for almost every $t \in I_{t_{0}}$. Thanks to the compactness of $I$, we can repeat such an argument a finite number of times and deduce that actually

$$
f^{*}(t, x(t))=f(t, x(t)) \quad \text { for a.e. } t \in I .
$$


As a consequence, one can prove condition (W) of Definition 2.4 for $f^{*}$ as follows: consider $\left(x_{n}(\cdot)\right)_{n \in \mathbb{N}}$ in $\mathcal{K}_{j}^{I}$ uniformly converging to $x(\cdot) \in \mathcal{K}_{j}^{I}$, then

$$
\begin{aligned}
\lim _{n \rightarrow \infty} \int_{I} f^{*}\left(t, x_{n}(t)\right) d t & =\lim _{n \rightarrow \infty} \int_{I} f\left(t, x_{n}(t)\right) d t \\
& =\int_{I} f(t, x(t)) d t=\int_{I} f^{*}(t, x(t)) d t .
\end{aligned}
$$

Therefore, $f$ and $f^{*}$ are two representatives of the same element of $\mathfrak{W} \Theta \mathfrak{C}\left(\mathbb{R}^{M}\right)$, because both are in $\mathfrak{W} \Theta \mathfrak{C}\left(\mathbb{R}^{M}\right)$ and only differ from each other on a negligible subset of $\mathbb{R} \times \mathbb{R}^{N}$.

Finally, we prove that $\bar{E}$ admits $L_{l o c}^{1}$-equicontinuous $m$-bounds. For each $f \in \bar{E}$ and any $j \in \mathbb{N}$, let $m_{f}^{j}$ be either, the $m$-bound of $f$ in $S^{j}$ if $f \in E$, or the $m$-bound given by (2.24) if $f \in \bar{E} \backslash E$, i.e. the absolutely continuous part of a limit measure.

Consider $j \in \mathbb{N}, r, \varepsilon>0$ and let $\delta=\delta(r, \varepsilon)>0$ be the one given by the $L_{l o c}^{1}$-equicontinuity of $S^{j+1}$. If $t, s \in[-r, r]$ with $s<t, t-s<\delta / 3$, and $\phi \in C_{C}^{+}$ is such that $\operatorname{supp} \phi \subset[s-\delta / 3, t+\delta / 3]$ and $\phi \equiv 1$ in $[s, t]$. Then, we have

$$
\begin{aligned}
\int_{s}^{t} m_{f}^{j}(u) d u & \leq \int_{\mathbb{R}} \phi(u) m_{f}^{j}(u) d u \leq \lim _{n \rightarrow \infty} \int_{\mathbb{R}} \phi(u) m_{f_{n}}^{j+1}(u) d u \\
& \leq \sup _{g \in E} \int_{s-\delta / 3}^{t+\delta / 3} m_{g}^{j+1}(u) d u<\varepsilon,
\end{aligned}
$$

and thus, taking the superior over the functions in $\bar{E}$ in the previous expression, one gets

$$
\sup _{f \in \bar{E}} \int_{s}^{t} m_{f}^{j}(u) d u<\varepsilon .
$$

Therefore, $\bar{E}$ admits $L_{l o c}^{1}$-equicontinuous $m$-bounds. Analogous reasonings apply to the remaining cases in (i) and (ii), whereas (iii) and (iv) are a direct consequence of (i) and (ii), respectively.

Remark 2.30. Notice that, since $\Theta \mathfrak{C}\left(\mathbb{R}^{M}\right) \subset \mathfrak{W} \Theta \mathfrak{C}\left(\mathbb{R}^{M}\right)$ and $\sigma_{\Theta}<\mathcal{T}_{\Theta}$, then the previous result can be directly applied in particular to the space $\left(\Theta \mathfrak{C}\left(\mathbb{R}^{M}\right), \mathcal{T}_{\Theta}\right)$. For $p>1$ one extend the result to $\left(\Theta \mathfrak{C}_{p}\left(\mathbb{R}^{M}\right), \mathcal{T}_{\Theta}\right)$ reasoning as in the proof of Proposition 2.27.

\subsection{Equivalence of the topologies}

As we have noticed before, all the introduced topologies can be induced on $\mathfrak{L} \mathfrak{C}_{p}\left(\mathbb{R}^{M}\right)$. As follows, we show that, given a subset $E$ of $\mathfrak{L} \mathfrak{C}_{p}\left(\mathbb{R}^{M}\right)\left(\right.$ resp. $\left.\mathfrak{L} \mathfrak{C}\left(\mathbb{R}^{M}\right)\right)$ with $L_{l o c}^{p}$-bounded (resp. $L_{l o c}^{1}$-bounded) $l$-bounds, all the introduced strong topologies (resp. weak topologies) coincide on $E$. Remarkably, such a rather weak 
assumption on the $l$-bounds allows to deduce such an important result for both, strong and weak topologies. A direct consequence is that, on such sets, one can simply use the topologies $\mathcal{T}_{D}$ and $\sigma_{D}$, respectively, to take the actual limits, but still change from one topology to another when it is required. Notice also that in many applications such assumption is trivially satisfied as, for example, when the $l$-bounds of a set $E$ are taken constant and bounded.

Theorem 2.31. Let $E$ be a set in $\mathfrak{L C}_{p}\left(\mathbb{R}^{M}\right)$ with $L_{\text {loc }}^{p}$-bounded l-bounds, then

$$
\left(E, \mathcal{T}_{1}\right)=\left(E, \mathcal{T}_{2}\right) \quad \text { and } \quad \operatorname{cls}_{\left(\mathfrak{S c}_{p}\left(\mathbb{R}^{M}\right), \mathcal{T}_{1}\right)}(E)=\operatorname{cls}_{\left(\mathfrak{S c}_{p}\left(\mathbb{R}^{M}\right), \mathcal{T}_{2}\right)}(E)
$$

where $\mathcal{T}_{1}$ and $\mathcal{T}_{2}$ are any of the previously introduced strong topologies.

Proof. From Proposition 2.27 we know that $\operatorname{cls}_{\left(\mathfrak{C C}_{p}\left(\mathbb{R}^{M}\right), \mathcal{T}\right)}(E) \subset \mathfrak{L C}_{p}$ for any strong topology $\mathcal{T}$ among the ones presented in Section 2.2. Moreover, due to relation (2.10), it suffices to prove that if $\left(f_{n}\right)_{n \in \mathbb{N}}$ is a sequence of elements of $E$ converging to some $f$ in $\left(\mathfrak{L C}_{p}\left(\mathbb{R}^{M}\right), \mathcal{T}_{D}\right)$, then $\left(f_{n}\right)_{n \in \mathbb{N}}$ converges to $f$ in $\left(\mathfrak{L C}_{p}\left(\mathbb{R}^{M}\right), \mathcal{T}_{B}\right)$. Fix a compact interval $I=\left[q_{1}, q_{2}\right]$, with $q_{1}, q_{2} \in \mathbb{Q}, j \in \mathbb{N}$ and, for any $n \in \mathbb{N}$, let $l_{n}^{j}(\cdot) \in L_{l o c}^{p}$ be the optimal $l$-bound of $f_{n}$ on $B_{j}$ (see Definition 2.1). By assumption, there exists $\rho>0$ such that

$$
\sup _{n \in \mathbb{N}} \int_{I}\left(l_{n}^{j}(s)\right)^{p} d s<\rho<\infty .
$$

Now, fix $\varepsilon>0$ and consider $\delta=\varepsilon /\left(3 \rho^{1 / p}\right)$. Since $B_{j} \subset \mathbb{R}^{N}$ is compact, and $D$ is dense in $\mathbb{R}^{N}$, there exist $x_{1}, \ldots x_{\nu} \in D$ such that $B_{j} \subset \bigcup_{i=1}^{\nu} B_{\delta}\left(x_{i}\right)$, where $B_{\delta}(x)$ denotes the closed ball of $\mathbb{R}^{N}$ of radius $\delta$ centered at $x \in \mathbb{R}^{N}$. For $i=1, \ldots, \nu$, let us consider continuous functions $\phi_{i}: \mathbb{R}^{N} \rightarrow[0,1]$ such that

$$
\operatorname{supp}\left(\phi_{i}\right) \subset B_{\delta}\left(x_{i}\right) \quad \text { and } \quad \sum_{i=1}^{\nu} \phi_{i}(x)=1 \quad \forall x \in B_{j},
$$

and define the functions

$$
f_{n}^{*}(t, x)=\sum_{i=1}^{\nu} \phi_{i}(x) f_{n}\left(t, x_{i}\right) \quad \text { and } \quad f^{*}(t, x)=\sum_{i=1}^{\nu} \phi_{i}(x) f\left(t, x_{i}\right) .
$$

Then, for any $x(\cdot) \in C\left(I, B_{j}\right)$ we have that

$$
\begin{aligned}
\| f_{n}(\cdot, x(\cdot)) & -f(\cdot, x(\cdot))\left\|_{p} \leq\right\| f_{n}(\cdot, x(\cdot))-f_{n}^{*}(\cdot, x(\cdot)) \|_{p} \\
& +\left\|f_{n}^{*}(\cdot, x(\cdot))-f^{*}(\cdot, x(\cdot))\right\|_{p}+\left\|f^{*}(\cdot, x(\cdot))-f(\cdot, x(\cdot))\right\|_{p} .
\end{aligned}
$$

Let us separately analyze each element in the sum on the right-hand side of 
equation (2.27). As regards the first one, we have that

$$
\begin{aligned}
\left\|f_{n}(\cdot, x(\cdot))-f_{n}^{*}(\cdot, x(\cdot))\right\|_{p}^{p} & =\int_{I}\left|\sum_{i=1}^{\nu} \phi_{i}(x(t))\left[f_{n}(t, x(t))-f_{n}\left(t, x_{i}\right)\right]\right|^{p} d t \\
& \leq \int_{I}\left(\sum_{i=1}^{\nu} \phi_{i}(x(t)) l_{n}^{j}(t)\left|x(t)-x_{i}\right|\right)^{p} d t \\
& \leq \int_{I}\left(\sum_{i=1}^{\nu} \phi_{i}(x(t)) l_{n}^{j}(t) \delta\right)^{p} d t \\
& =\frac{1}{\rho}\left(\frac{\varepsilon}{3}\right)^{p} \int_{I}\left(l_{n}^{j}(t)\right)^{p} d t \leq\left(\frac{\varepsilon}{3}\right)^{p} .
\end{aligned}
$$

As regards the third element of the sum in (2.27), recall that, due to the Proposition 2.27 , the $l$-bound $\bar{l}^{j}(\cdot) \in L_{l o c}^{p}$ on $B_{j}$ for $f$ satisfies

$$
\int_{I}\left(\bar{l}^{j}(s)\right)^{p} d s<\rho .
$$

Therefore, reasoning as in (2.28), we obtain that

$$
\left\|f^{*}(\cdot, x(\cdot))-f(\cdot, x(\cdot))\right\|_{p} \leq \frac{\varepsilon}{3},
$$

and notice that both, (2.28) and (2.29), are independent of $x(\cdot) \in C\left(I, B_{j}\right)$.

Finally, since $\left(f_{n}\right)_{n \in \mathbb{N}}$ converges to $f$ in $\left(\mathfrak{L} \mathfrak{C}_{p}\left(\mathbb{R}^{M}\right), \mathcal{T}_{D}\right)$, consider $n$ big enough so that

$$
\left\|f_{n}\left(\cdot, x_{i}\right)-f\left(\cdot, x_{i}\right)\right\|_{p}<\varepsilon /(3 \nu), \quad \text { for any } i=1, \ldots, \nu .
$$

Then, from the expressions $(2.26)$ and the fact that $\phi_{i}(x) \leq 1$ for each $x \in \mathbb{R}^{N}$ we deduce that

$$
\left\|f_{n}^{*}(\cdot, x(\cdot))-f^{*}(\cdot, x(\cdot))\right\|_{p} \leq \sum_{i=1}^{\nu}\left\|f_{n}\left(\cdot, x_{i}\right)-f\left(\cdot, x_{i}\right)\right\|_{p} \leq \frac{\varepsilon}{3} .
$$

Gathering together (2.28), (2.29) and (2.30), we obtain the result.

When dealing with a function in $\mathfrak{L} \mathfrak{C}_{p}\left(\mathbb{R}^{M}\right)$ with $L_{\text {loc }}^{p}$-bounded l-bounds, the previous theorem provides, as a corollary, a condition of equivalence of the hulls with respect to the introduced strong topologies.

Corollary 2.32. Let $f$ be a function in $\mathfrak{L C}_{p}\left(\mathbb{R}^{M}\right)$ with $L_{\text {loc }}^{p}$-bounded l-bounds, then

$$
\operatorname{Hull}_{\left(\mathfrak{S C}_{p}\left(\mathbb{R}^{M}\right), \mathcal{T}_{1}\right)}(f)=\operatorname{Hull}_{\left(\mathfrak{S C}_{p}\left(\mathbb{R}^{M}\right), \mathcal{T}_{2}\right)}(f),
$$

where $\mathcal{T}_{1}$ and $\mathcal{T}_{2}$ are any of the previously introduced strong topologies.

A result similar to Theorem 2.31 can be obtained for weak topologies. 
Theorem 2.33. Let $E$ be a set in $\mathfrak{L} \mathfrak{C}\left(\mathbb{R}^{M}\right)$ with $L_{\text {loc }}^{1}$-bounded l-bounds, $\Theta$ be any suitable set of moduli of continuity as in Definition 2.3 and $D$ any dense and countable subset of $\mathbb{R}^{N}$. Then

$$
\left(E, \sigma_{D}\right)=\left(E, \sigma_{\Theta}\right) \quad \text { and } \quad \operatorname{cls}_{\left(\mathfrak{S C}\left(\mathbb{R}^{M}\right), \sigma_{D}\right)}(E)=\operatorname{cls}_{\left(\mathfrak{S C C}\left(\mathbb{R}^{M}\right), \sigma_{\Theta}\right)}(E) .
$$

Proof. Fix $D$, dense and countable subset of $\mathbb{R}^{N}$, and $\Theta$, suitable set of moduli of continuity as in Definition 2.3. Thanks to Proposition 2.26, we know that $\operatorname{cls}_{\left(\mathfrak{S} \mathfrak{C}\left(\mathbb{R}^{M}\right), \sigma_{D}\right)}(E) \subset \mathfrak{L} \mathfrak{C}\left(\mathbb{R}^{M}\right)$. We will complete the proof in two steps.

Step 1. Consider a set $E_{1}$ with $L_{l o c}^{1}$-bounded $m$-bounds and $L_{l o c}^{1}$-bounded l-bounds. Let $\left(f_{n}\right)_{n \in \mathbb{N}}$ be a sequence of elements of $E_{1}$ converging to some $f$ in $\left(\mathfrak{L} \mathfrak{C}\left(\mathbb{R}^{M}\right), \sigma_{D}\right)$. We shall prove that $\left(f_{n}\right)_{n \in \mathbb{N}}$ converges to $f$ in $\left(\mathfrak{L} \mathfrak{C}\left(\mathbb{R}^{M}\right), \sigma_{\Theta}\right)$. Fix a compact interval $I=\left[q_{1}, q_{2}\right]$, with $q_{1}, q_{2} \in \mathbb{Q}, j \in \mathbb{N}$ and, for any $n \in \mathbb{N}$, let $m_{n}^{j}(\cdot), l_{n}^{j}(\cdot) \in L_{l o c}^{1}$ be respectively the optimal $m$-bound and the optimal $l$-bound of $f_{n}$ on $B_{j}$. By the $L_{l o c}^{1}$-boundedness of the $l$-bounds, there is a $\rho>0$ such that

$$
\sup _{n \in \mathbb{N}} \int_{I} l_{n}^{j}(s) d s<\rho<\infty .
$$

Fix $\varepsilon>0$ and consider $\delta=\varepsilon / 3 \rho$. Since $B_{j} \subset \mathbb{R}^{N}$ is compact, and $D$ is dense in $\mathbb{R}^{N}$, there exist $x_{1}, \ldots x_{\nu} \in D$ such that $B_{j} \subset \bigcup_{i=1}^{\nu} B_{\delta}\left(x_{i}\right)$, where $B_{\delta}(x)$ denotes the closed ball of $\mathbb{R}^{N}$ of radius $\delta$ centered at $x \in \mathbb{R}^{N}$. For $i=1, \ldots, \nu$, let us consider the continuous functions $\phi_{i}: \mathbb{R}^{N} \rightarrow[0,1]$, and the functions

$$
f_{n}^{*}: \mathbb{R} \times \mathbb{R}^{N} \rightarrow \mathbb{R}^{M}, n \in \mathbb{N}, \quad \text { and } \quad f^{*}: \mathbb{R} \times \mathbb{R}^{N} \rightarrow \mathbb{R}^{M},
$$

defined exactly as in (2.25) and (2.26), respectively. Denoted by $\mathcal{K}_{j}^{I}$ the compact subset of $C\left(I, B_{j}\right)$ admitting $\theta_{j}^{I} \in \Theta$ as a modulus of continuity, one has that for any $x(\cdot) \in \mathcal{K}_{j}^{I}$

$$
\begin{aligned}
& \left|\int_{I}\left[f_{n}(t, x(t))-f(t, x(t))\right] d t\right| \leq\left|\int_{I}\left[f_{n}(t, x(t))-f_{n}^{*}(t, x(t))\right] d t\right| \\
& \quad+\left|\int_{I}\left[f_{n}^{*}(t, x(t))-f^{*}(t, x(t))\right] d t\right|+\left|\int_{I}\left[f^{*}(t, x(t))-f(t, x(t))\right] d t\right| .
\end{aligned}
$$

Let us separately analyze each element in the sum on the right-hand side of equation (2.31). As regards the first one, we have that

$$
\begin{aligned}
\mid \int_{I}\left[f_{n}(t,\right. & \left.x(t))-f_{n}^{*}(t, x(t))\right] d t \mid \\
& =\left|\int_{I} \sum_{i=1}^{\nu} \phi_{i}(x(t))\left[f_{n}(t, x(t))-f_{n}\left(t, x_{i}\right)\right] d t\right| \\
& \leq \int_{I} \sum_{i=1}^{\nu} \phi_{i}(x(t)) l_{n}^{j}(t)\left|x(t)-x_{i}\right| d t \\
& \leq \int_{I} \sum_{i=1}^{\nu} \phi_{i}(x(t)) l_{n}^{j}(t) \delta d t=\frac{\varepsilon}{3 \rho} \int_{I} l_{n}^{j}(t) d t \leq \frac{\varepsilon}{3} .
\end{aligned}
$$


Similar reasonings apply to the third element of the sum in (2.31): in particular, recall that, due to Proposition 2.26, the $l$-bound for $f$ on $B_{j}$, namely $\bar{l}^{j}(\cdot) \in L_{l o c}^{1}$, satisfies

$$
\int_{I} \bar{l}^{j}(s) d s<\rho<\infty .
$$

Finally, let us deal with the remaining integral in (2.31). By the uniform continuity of the functions $\phi_{i}(\cdot)$ on $B_{j}$, and recalling that all $x(\cdot) \in \mathcal{K}_{j}^{I}$ share the same modulus of continuity, we have that for the given $\varepsilon>0$ there exists $\delta>0$ such that for all $i \in\{1, \ldots, \nu\}$ one has

$$
\forall s, t \in I, \forall x(\cdot) \in \mathcal{K}_{j}^{I}: \quad|s-t|<\delta \Rightarrow\left|\phi_{i}(x(s))-\phi_{i}(x(t))\right|<\frac{\varepsilon}{9 \nu \rho_{m}},
$$

where

$$
\rho_{m}:=\max \left\{\int_{I} m^{j}(t) d t, \sup _{n \in \mathbb{N}} \int_{I} m_{n}^{j}(t) d t\right\}<\infty,
$$

and $m_{j}(\cdot) \in L_{l o c}^{1}$ denotes the optimal $m$-bound for $f$ on $B^{j}$ whose existence is guaranteed by Proposition 2.26. In particular, the constant $\rho_{m}$ is well defined thanks to the $L_{l o c}^{1}$-boundedness of the $m$-bounds of the functions in $E_{1}$. Thus, let us consider a $\delta$-partition of $I$, i.e. $\tau_{1}, \ldots, \tau_{\eta} \in I \cap \mathbb{Q}$ such that $I=\left[\tau_{1}, \tau_{\eta}\right]$ and $0<\tau_{k+1}-\tau_{k}<\delta$, for any $k=1, \ldots, \eta-1$, and a function

$$
\bar{\phi}_{i}: \mathcal{K}_{j}^{I} \rightarrow L^{\infty}(I, \mathbb{R}) \quad \text { defined by } \quad \bar{\phi}_{i}(x(t))=\sum_{k=1}^{\eta} \phi_{i}\left(x\left(\tau_{k}\right)\right) \chi_{\left(\tau_{k}, \tau_{k+1}\right]}(t)
$$

Notice that, for any $x(\cdot) \in \mathcal{K}_{j}^{I}$ and any $i=1, \ldots, \nu$ one has

$$
\left\|\phi_{i}(x(\cdot))-\bar{\phi}_{i}(x(\cdot))\right\|_{L^{\infty}(I)}<\frac{\varepsilon}{9 \nu \rho_{m}},
$$

Now, we can write

$$
\begin{aligned}
\left|\int_{I}\left[f_{n}^{*}(t, x(t))-f^{*}(t, x(t))\right] d t\right| \\
\leq \sum_{i=1}^{\nu}\left|\int_{I} \phi_{i}(x(t))\left[f_{n}\left(t, x_{i}\right)-f\left(t, x_{i}\right)\right] d t\right| \\
\leq \sum_{i=1}^{\nu}\left|\int_{I} \bar{\phi}_{i}(x(t))\left[f_{n}\left(t, x_{i}\right)-f\left(t, x_{i}\right)\right] d t\right|+ \\
\quad+\sum_{i=1}^{\nu} \int_{I}\left|f_{n}\left(t, x_{i}\right)\right|\left|\phi_{i}(x(t))-\bar{\phi}_{i}(x(t))\right| d t+ \\
\quad+\sum_{i=1}^{\nu} \int_{I}\left|f\left(t, x_{i}\right)\right|\left|\bar{\phi}_{i}(x(t))-\phi_{i}(x(t))\right| d t \\
\leq \sum_{i=1}^{\nu}\left[\sum_{k=1}^{\eta} \phi_{i}\left(x\left(\tau_{k}\right)\right)\left|\int_{\tau_{k}}^{\tau_{k+1}}\left[f_{n}\left(t, x_{i}\right)-f\left(t, x_{i}\right)\right] d t\right|+\right. \\
\left.\quad+2 \rho_{m}\left\|\phi_{i}(x(\cdot))-\bar{\phi}_{i}(x(\cdot))\right\|_{L^{\infty}(I)}\right] .
\end{aligned}
$$


By the convergence of $\left(f_{n}\right)_{n \in \mathbb{N}}$ to $f$ in $\left(\mathfrak{L C}\left(\mathbb{R}^{M}\right), \sigma_{D}\right)$ and considering that we are only using a finite number of points $x_{i}$, with $i=1, \ldots, \nu$, there exists $n_{0} \in \mathbb{N}$ such that, if $n>n_{0}$, then for all $i=1, \ldots, \nu$ and for all $k=1, \ldots, \eta$ one has

$$
\left|\int_{\tau_{k}}^{\tau_{k+1}}\left[f_{n}\left(t, x_{i}\right)-f\left(t, x_{i}\right)\right] d t\right|<\frac{\varepsilon}{9 \nu \eta} .
$$

Thus, for $n>n_{0},(2.33)$ becomes

$$
\left|\int_{I}\left[f_{n}^{*}(t, x(t))-f^{*}(t, x(t))\right] d t\right|<\frac{\varepsilon}{9}+\frac{2 \nu \rho_{m} \varepsilon}{9 \nu \rho_{m}}=\frac{\varepsilon}{3} .
$$

From (2.31), (2.32) and (2.34) we obtain that the sequence $\left(f_{n}\right)_{n \in \mathbb{N}}$ converges to $f$ in $\left(\mathfrak{L} \mathfrak{C}\left(\mathbb{R}^{M}\right), \sigma_{\Theta}\right)$. Consequently, the topologies of type $\sigma_{D}$ and $\sigma_{\Theta}$ are equivalent on $E_{1}$.

Step 2. Now, consider $E$ as in the assumptions, i.e. $E \subset \mathfrak{L} \mathfrak{C}\left(\mathbb{R}^{M}\right)$ with (just) $L_{\text {loc }}^{1}$-bounded $l$-bounds, and, again, let $\left(f_{n}\right)_{n \in \mathbb{N}}$ be a sequence of elements of $E$ converging to some $f$ in $\left(\mathfrak{L} \mathfrak{C}\left(\mathbb{R}^{M}\right), \sigma_{D}\right)$. We shall prove that $\left(f_{n}\right)_{n \in \mathbb{N}}$ converges to $f$ in $\left(\mathfrak{L} \mathfrak{C}\left(\mathbb{R}^{M}\right), \sigma_{\Theta}\right)$. In particular consider $x_{0} \in B_{1} \cap D$ and define the functions

$$
h(t, x)=f(t, x)-f\left(t, x_{0}\right) \quad \text { and } \quad h_{n}(t, x)=f_{n}(t, x)-f_{n}\left(t, x_{0}\right), \forall n \in \mathbb{N} .
$$

Notice that the set $\left\{h_{n} \mid n \in \mathbb{N}\right\} \cup\{h\}$ has $L_{l o c}^{1}$-bounded $m$-bounds. Indeed, for any $j \in \mathbb{N}$, considered $x \in B_{j}$ one has that for any $n \in \mathbb{N}$

$$
\left|h_{n}(t, x)\right|=\left|f_{n}(t, x)-f_{n}\left(t, x_{0}\right)\right| \leq l_{n}^{j}(t)\left|x-x_{0}\right| \leq(j+1) l_{n}^{j}(t) \quad \text { for a.e. } t \in \mathbb{R},
$$

where $l_{n}^{j}(\cdot) \in L_{l o c}^{1}$ is the optimal $l$-bound for $f_{n}$ on $B_{j}$. Additionally, one can repeat analogous arguments for the function $h$ obtaining

$$
|h(t, x)| \leq(j+1) l^{j}(t) \text { for a.e. } t \in \mathbb{R},
$$

where $l^{j}(\cdot) \in L_{l o c}^{1}$ is the optimal $l$-bound for $f$ on $B_{j}$. Thus, the $L_{l o c}^{1}$-boundedness of the $l$-bounds for the set $E$ gives $L_{l o c}^{1}$-bounded $m$-bounds for $\left\{h_{n} \mid n \in \mathbb{N}\right\} \cup\{h\}$. Moreover, we have that the same set has also $L_{l o c}^{1}$-bounded $l$-bounds. Indeed, for any $j \in \mathbb{N}$, considered $x, y \in B_{j}$ one has that for every $n \in \mathbb{N}$

$$
\left|h_{n}(t, x)-h_{n}(t, y)\right|=\left|f_{n}(t, x)-f_{n}(t, y)\right| \leq l_{n}^{j}(t)|x-y| \quad \text { for a.e. } t \in \mathbb{R},
$$

and one can repeat analogous arguments for the function $h$ obtaining

$$
|h(t, x)-h(t, y)| \leq l^{j}(t)|x-y| \quad \text { for a.e. } t \in \mathbb{R} .
$$

Therefore, from the $L_{l o c}^{1}$-boundedness of the $l$-bounds for the set $E$ one has that $\left\{h_{n} \mid n \in \mathbb{N}\right\} \cup\{h\}$ also has $L_{l o c}^{1}$-bounded $l$-bounds. Finally notice that $\left(h_{n}\right)_{n \in \mathbb{N}}$ converges to $h$ in $\left(\mathfrak{L} \mathfrak{C}\left(\mathbb{R}^{M}\right), \sigma_{D}\right)$. Indeed, for each $x \in D$ and for each interval $I=\left[q_{1}, q_{2}\right]$, with $q_{1}, q_{2} \in \mathbb{Q}$ we have

$$
\left|\int_{I}\left[h_{n}(t, x)-h(t, x)\right] d t\right| \leq\left|\int_{I}\left[f_{n}(t, x)-f(t, x)\right] d t\right|+\left|\int_{I}\left[f\left(t, x_{0}\right)-f_{n}\left(t, x_{0}\right)\right] d t\right|
$$


and since $\left(f_{n}\right)_{n \in \mathbb{N}}$ converges to $f$ in $\left(\mathfrak{L} \mathfrak{C}\left(\mathbb{R}^{M}\right), \sigma_{D}\right)$, then the integrals on the right-hand side of the previous inequality go to zero as $n \rightarrow \infty$. Therefore, the assumptions of step 1 apply to the set $E_{1}=\left\{h_{n} \mid n \in \mathbb{N}\right\} \cup\{h\}$ and thus one has that $\left(h_{n}\right)_{n \in \mathbb{N}}$ converges to $h$ in $\left(\mathfrak{L} \mathfrak{C}\left(\mathbb{R}^{M}\right), \sigma_{\Theta}\right)$. Hence, for each interval $I=\left[q_{1}, q_{2}\right]$, with $q_{1}, q_{2} \in \mathbb{Q}$ and for each $j \in \mathbb{N}$ one has

$$
\begin{aligned}
\sup _{x(\cdot) \in \mathcal{K}_{j}^{I}} \mid & \int_{I}\left[f_{n}(t, x(t))-f(t, x(t))\right] d t \mid \\
& \leq \sup _{x(\cdot) \in \mathcal{K}_{j}^{I}}\left|\int_{I}\left[h_{n}(t, x(t))-h(t, x(t))\right] d t\right|+\left|\int_{I}\left[f_{n}\left(t, x_{0}\right)-f\left(t, x_{0}\right)\right] d t\right|,
\end{aligned}
$$

and the right-hand side goes to zero as $n \rightarrow \infty$ because $\left(h_{n}\right)_{n \in \mathbb{N}}$ converges to $h$ in $\left(\mathfrak{L} \mathfrak{C}\left(\mathbb{R}^{M}\right), \sigma_{\Theta}\right)$ and $\left(f_{n}\right)_{n \in \mathbb{N}}$ converges to $f$ in $\left(\mathfrak{L} \mathfrak{C}\left(\mathbb{R}^{M}\right), \sigma_{D}\right)$, which implies that $\left(f_{n}\right)_{n \in \mathbb{N}}$ converges to $f$ in $\left(\mathfrak{L} \mathfrak{C}\left(\mathbb{R}^{M}\right), \sigma_{\Theta}\right)$ and, as a consequence, all the topologies of type $\sigma_{D}$ and $\sigma_{\Theta}$ coincide on $E$.

Thanks to the previous theorem, if one has a function $f$ in $\mathfrak{L C}_{p}\left(\mathbb{R}^{M}\right)$ with $L_{l o c}^{1}$-bounded $l$-bounds, then one has the equivalence of the hulls of $f$ with respect to the introduced weak topologies.

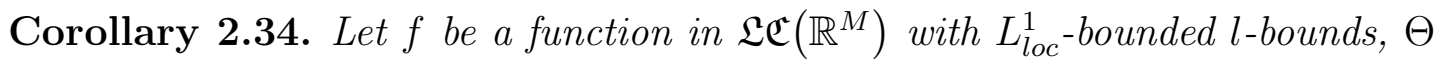
be any suitable set of moduli of continuity as in Definition 2.3 and $D$ any dense and countable subset of $\mathbb{R}^{N}$. Then,

$$
\operatorname{Hull}_{\left(\mathfrak{S C}\left(\mathbb{R}^{M}\right), \sigma_{D}\right)}(f)=\operatorname{Hull}_{\left(\mathfrak{S C}\left(\mathbb{R}^{M}\right), \sigma_{\Theta}\right)}(f) .
$$

\subsection{Relative compactness in $\mathfrak{L} \mathfrak{C}_{p}$}

The notion of compactness together with the continuity of the flow generated by a dynamical system allow to infer important information on the system and its behavior. As an example, thanks to Krylov-Bogoljubov theorem, a compact hull implies the existence of an invariant measure for the base flow and allows to use tools of ergodic theory in the study of the solutions. Therefore, it seems natural to us to look into the conditions that characterize the relatively compact subsets of the space $\mathfrak{L} \mathfrak{C}_{p}\left(\mathbb{R}^{M}\right)$.

In this section, we firstly address the relative compactness in $\mathfrak{L C}_{p}\left(\mathbb{R}^{M}\right)$ with respect to the strong topologies introduced in Section 2.2 and then recall the results in the literature for the weak topologies in $\mathfrak{L} \mathfrak{C}\left(\mathbb{R}^{M}\right)$.

\subsubsection{Strong topologies}

A characterization of compactness in $L_{l o c}^{p}\left(\mathbb{R}^{M}\right)$, when $1 \leq p<\infty$, has been given in [52, Theorem 1 p.133], where it is proved that $E \subset L_{l o c}^{p}\left(\mathbb{R}^{M}\right)$ is relatively compact if and only if both the following conditions hold: 
(i) for every compact interval $I \subset \mathbb{R}$ there exists a constant $c=c(I)$ such that

$$
\left[\int_{I}|f(t)|^{p} d t\right]^{1 / p} \leq c, \quad \text { for every } f \in E,
$$

(ii) and for every $\varepsilon>0$ and for every compact interval $I \subset \mathbb{R}$ there exists a $\delta=\delta(\varepsilon, I)>0$ such that

$$
|\tau| \leq \delta \quad \Rightarrow \quad\left[\int_{I}|f(t+\tau)-f(t)|^{p} d t\right]^{1 / p} \leq \varepsilon \quad \text { for every } f \in E
$$

A sufficient condition for the relative compactness of a set $E$ in $\left(\mathfrak{L} \mathfrak{C}_{p}\left(\mathbb{R}^{M}\right), T_{B}\right)$ is also given in the same reference. Next, we characterize such a compactness under the assumption that the set $E$ admits $L_{l o c}^{p}$-bounded $l$-bounds.

Theorem 2.35. Let $E \subset \mathfrak{L C}_{p}\left(\mathbb{R}^{M}\right)$ admit $L_{\text {loc }}^{p}$-bounded l-bounds, $\mathcal{T}$ be any of the strong topologies introduced in Section 2.2, and $D$ be a countable dense subset of $\mathbb{R}^{N}$. The following statements are equivalent.

(i) The space $(E, \mathcal{T})$ is relatively compact.

(ii) For any fixed $x \in D$ the set $\left\{f_{x}(\cdot)=f(\cdot, x) \mid f \in E\right\}$ is relatively compact in $L_{l o c}^{p}\left(\mathbb{R}^{M}\right)$.

Proof. Firstly, recall that, since $E$ has $L_{l o c}^{p}$-bounded $l$-bounds, then all the strong topologies introduced in Section 2.2 are equivalent thanks to Theorem 2.31, and thus we will work with $\left(E, \mathcal{T}_{D}\right)$. (i) $\Rightarrow$ (ii) is straightforward.

(ii) $\Rightarrow$ (i). Consider a sequence $\left(f_{n}\right)_{n \in \mathbb{N}}$ in $E$, fix $j \in \mathbb{N}$ and, for any $n \in \mathbb{N}$, let $l_{n}^{j}(\cdot)$ be the optimal $l$-bound for $f_{n}$ on $B_{j}$. Moreover, let $D_{j}$ be the set $D \cap B_{j}$. By hypothesis, for any $x \in D_{j}$ the set $\left\{f_{n}(\cdot, x) \mid n \in \mathbb{N}\right\}$ is relatively compact in $L_{l o c}^{p}$; as a consequence, there exists a function $f_{x}(\cdot) \in L_{l o c}^{p}\left(\mathbb{R}^{M}\right)$ such that, up to a subsequence

$$
f_{n}(\cdot, x) \stackrel{n \rightarrow \infty}{\longrightarrow} f_{x}(\cdot), \quad \text { in } L_{l o c}^{p}\left(\mathbb{R}^{M}\right)
$$

and thus also almost everywhere. Recalling that $D$ is countable and using a diagonal argument, we obtain a subsequence of $\left(f_{n}\right)_{n \in \mathbb{N}}$, which we keep denoting with the same indexes, such that

$$
f_{n}(t, x) \stackrel{n \rightarrow \infty}{\longrightarrow} f(t, x) \text { for a.e. } t \in \mathbb{R}, \forall x \in D_{j},
$$

where for every $x \in D_{j}, f(t, x)=f_{x}(t)$. Moreover, by assumption, the set $\left\{l_{n}^{j}(\cdot) \mid\right.$ $n \in \mathbb{N}\}$ is bounded in $L_{l o c}^{p}$ and thus, reasoning as in the proofs of Proposition 2.26 and 2.27, we obtain a function $l^{j}(\cdot) \in L_{l o c}^{p}$ such that for any $x, y \in D_{j}$ the following inequality holds

$$
|f(t, x)-f(t, y)| \leq l^{j}(t)|x-y| \quad \text { for a.e. } t \in \mathbb{R} .
$$


A continuous extension of $f$ to the entire ball $B_{j}$ is given by

$$
f(t, x)=\lim _{n \rightarrow \infty} f\left(t, x_{n}\right) \quad \text { whenever } x \in B_{j},\left(x_{n}\right)_{n \in \mathbb{N}} \text { in } D_{j} \text {, and } x_{n} \rightarrow x .
$$

The definition is well-posed; indeed if $\left(x_{n}\right)_{n \in \mathbb{N}}$ and $\left(y_{n}\right)_{n \in \mathbb{N}}$ are sequences in $D_{j}$ such that $x_{n} \rightarrow x$ and $y_{n} \rightarrow x$, then

$$
\left|f\left(t, x_{n}\right)-f\left(t, y_{n}\right)\right| \leq l^{j}(t)\left|x_{n}-y_{n}\right| \text { for a.e. } t \in \mathbb{R},
$$

and the right-hand side goes to zero as $n \rightarrow \infty$, for almost every $t \in \mathbb{R}$. It is straightforward to prove that $l^{j}(\cdot)$ keeps being a Lipschitz coefficient for the function $f$ defined on the whole ball $B_{j}$. Eventually, we check the properties $(\mathbf{C} 1)$ and (C2). For every $j, n \in \mathbb{N}$, define

$$
F_{n}(t, x)=n \int_{t}^{t+1 / n} f(s, x) d s \quad \text { and } \quad L_{n}^{j}(t)=n \int_{t}^{t+1 / n} l^{j}(s) d s .
$$

Notice that for any $n \in \mathbb{N}$ one has that $L_{n}^{j}(\cdot)$ is continuous, and, for any $x \in \mathbb{R}^{N}$, also $F_{n}(\cdot, x)$ is continuous. Moreover, for any $t \in \mathbb{R}$ we also have

$$
\begin{aligned}
\left|F_{n}\left(t, x_{1}\right)-F_{n}\left(t, x_{2}\right)\right| & \leq n \int_{t}^{t+1 / n}\left|f\left(s, x_{1}\right)-f\left(s, x_{2}\right)\right| d s \\
& \leq\left|x_{1}-x_{2}\right| n \int_{t}^{t+1 / n} l^{j}(s) d s=L_{n}^{j}(t)\left|x_{1}-x_{2}\right|,
\end{aligned}
$$

for all $n \in \mathbb{N}$ and $x_{1}, x_{2} \in B_{j}$. In fact, for any $n \in \mathbb{N}$ the function $F_{n}(\cdot, \cdot)$ is jointly continuous. To see that, consider $t_{k} \rightarrow t$ and $x_{k} \rightarrow x$ as $k \rightarrow \infty$, and $j \in \mathbb{N}$ such that $\left|x_{k}\right|<j$ for all $k \in \mathbb{N}$. Then, using the triangular inequality, (2.37) and the $m$-bound of $f_{n}$ on $B_{j}$, we have that

$$
\begin{aligned}
\left|F_{n}\left(t_{k}, x_{k}\right)-F_{n}(t, x)\right| & \leq\left|F_{n}\left(t_{k}, x_{k}\right)-F_{n}\left(t_{k}, x\right)\right|+\left|F_{n}\left(t_{k}, x\right)-F_{n}(t, x)\right| \\
& \leq L_{n}^{j}\left(t_{k}\right)\left|x_{k}-x\right|+n \int_{I_{k}} m_{f_{n}}^{j}(t) d t,
\end{aligned}
$$

where $I_{k}$ is either $\left[t_{k}, t\right]$ or $\left[t, t_{k}\right]$. Hence, by the continuity of Lebesgue integral, one has that the right-hand side of the previous chain of inequalities goes to zero as $k \rightarrow \infty$, and thus $F_{n}(\cdot, \cdot)$ is jointly continuous. Notice also that there exists a Borel set $R_{0} \subset \mathbb{R}$, with meas $\mathbb{R}\left(\mathbb{R} \backslash R_{0}\right)=0$ such that,

$$
F_{n}(t, x) \stackrel{n \rightarrow \infty}{\longrightarrow} f(t, x) \quad \text { and } \quad L_{n}^{j}(t) \stackrel{n \rightarrow \infty}{\longrightarrow} l^{j}(t),
$$

for every $(t, x) \in R_{0} \times D$ and for every $j \in \mathbb{N}$. However, the convergence in (2.38) can be extended to $R_{0} \times \mathbb{R}^{N}$. Consider $x \in \mathbb{R}^{N},\left(x_{i}\right)_{i \in \mathbb{N}} \subset D$ converging to $x$ and fix $i \in \mathbb{N}$. Then, for any $t \in R_{0}$ one has

$$
\begin{aligned}
\left|F_{n}(t, x)-f(t, x)\right| \leq & \left|F_{n}(t, x)-F_{n}\left(t, x_{i}\right)\right| \\
& \quad+\left|F_{n}\left(t, x_{i}\right)-f\left(t, x_{i}\right)\right|+\left|f\left(t, x_{i}\right)-f(t, x)\right| \\
\leq & L_{n}^{j}(t)\left|x-x_{i}\right|+\left|F_{n}\left(t, x_{i}\right)-f\left(t, x_{i}\right)\right|+l^{j}(t)\left|x-x_{i}\right| .
\end{aligned}
$$


Therefore, from (2.38) and taking the limit as $n \rightarrow \infty$, and from the arbitrariness of $i \in \mathbb{N}$ we obtain the result. Hence, $f \chi_{R_{0} \times \mathbb{R}^{N}}$ is a Borel function, i.e. it satisfies (C1). Finally, let us fix $x_{0} \in B_{1}$, and assume without loss of generality that $\left|f\left(t, x_{0}\right)\right|<\infty$. For every $x \in B_{j}$, we have that

$$
|f(t, x)| \leq\left|f\left(t, x_{0}\right)\right|+l^{j}(t)\left|x-x_{0}\right|=: m_{f}^{j}(t),
$$

for a.e. $t \in \mathbb{R}$. Thus, $f$ admits $m$-bounds, i.e. $f$ satisfies (C2). As a consequence, $\left(f_{n}\right)_{n \in \mathbb{N}}$ converges to $f$ in $\left(\mathfrak{L C}_{p}\left(\mathbb{R}^{M}\right), \mathcal{T}_{D}\right)$, which in turn implies $f \in$ $\operatorname{cls}_{\left(\mathfrak{L C}_{p}\left(\mathbb{R}^{M}\right), \mathcal{T}_{D}\right)}(E)$, and $\operatorname{cls}_{\left(\mathfrak{L C}_{p}\left(\mathbb{R}^{M}\right), \mathcal{T}_{D}\right)}(E)$ is compact in $\left(\mathfrak{L C}_{p}\left(\mathbb{R}^{M}\right), \mathcal{T}_{D}\right)$, which concludes the proof.

As a corollary of Theorem 2.35 and of the conditions given in (2.35) and (2.36), we obtain a characterization of the compactness of $\operatorname{Hull}_{\left(\mathfrak{L C}_{p}\left(\mathbb{R}^{M}\right), \mathcal{T}\right)}(f)$ when $f \in$ $\mathfrak{L C}_{p}$ admits $L_{l o c}^{p}$-bounded l-bounds.

Corollary 2.36. Let $f \in \mathfrak{L C}_{p}\left(\mathbb{R}^{M}\right)$ admit $L_{\text {loc }}^{p}$-bounded l-bounds, $\mathcal{T}$ be any of the previously introduced strong topologies, and $D$ be a countable dense subset of $\mathbb{R}^{N}$. The following statements are equivalent.

(i) $\operatorname{Hull}_{\left(\mathfrak{L C}_{p}\left(\mathbb{R}^{M}\right), \mathcal{T}\right)}(f)$ is compact.

(ii) For every $x \in D$ the map $\mathbb{R} \rightarrow L_{l o c}^{p}\left(\mathbb{R}^{M}\right), t \mapsto f_{t}(\cdot, x)$ is bounded and uniformly continuous.

Proof. (i) $\Rightarrow$ (ii). If $\operatorname{Hull}_{\left(\mathfrak{L C}_{p}\left(\mathbb{R}^{M}\right), \mathcal{T}\right)}(f)$ is compact, then from Theorem 2.35(i) we have that for every $x \in D$ the set

$$
H_{x}=\left\{g(\cdot, x) \mid g \in \operatorname{Hull}_{\left(\mathfrak{L C}_{p}\left(\mathbb{R}^{M}\right), \mathcal{T}\right)}(f)\right\} \quad \text { is relatively compact in } L_{\text {loc }}^{p}\left(\mathbb{R}^{M}\right) .
$$

Since for every $x \in D$ one has $\left\{f_{t}(\cdot, x) \mid t \in \mathbb{R}\right\} \subset H_{x}$, then one also has that $\left\{f_{t}(\cdot, x) \mid t \in \mathbb{R}\right\}$ is relatively compact in $L_{\text {loc }}^{p}\left(\mathbb{R}^{M}\right)$. As a consequence the conditions given in (2.35) and (2.36) hold true for $\left\{f_{t}(\cdot, x) \mid t \in \mathbb{R}\right\}$. Particularly, for every $x \in D$ the map

$$
\mathbb{R} \rightarrow L_{l o c}^{p}\left(\mathbb{R}^{M}\right), \quad t \mapsto f_{t}(\cdot, x)
$$

is bounded in $L_{l o c}^{p}$ thanks to (2.35). Therefore, in order to complete the proof, we only need to check the uniform continuity in $L_{l o c}^{p}$. Fix $x \in D, I \subset \mathbb{R}$ and $\varepsilon>0$, and let $\delta=\delta(x, \varepsilon, I)>0$ be the one given in (2.36). Now, consider $0 \leq \tau<\delta$ and $t \in \mathbb{R}$. Noticing that the condition in (2.36) is uniform on the functions in $\left\{f_{t}(\cdot, x) \mid t \in \mathbb{R}\right\}$, one has

$$
\left[\int_{I}\left|f_{t+\tau}(s, x)-f_{t}(s, x)\right|^{p} d s\right]^{1 / p} \leq \sup _{t \in \mathbb{R}}\left[\int_{I}\left|\left(f_{t}\right)_{\tau}(s, x)-f_{t}(s, x)\right|^{p} d s\right]^{1 / p}<\varepsilon,
$$

which concludes this part of the proof. 
(ii) $\Rightarrow$ (i). One immediately has that for every $x \in D$, the conditions given in (2.35) and (2.36) hold true for $\left\{f_{t}(\cdot, x) \mid t \in \mathbb{R}\right\}$ and hence

$$
\left\{f_{t}(\cdot, x) \mid t \in \mathbb{R}\right\} \quad \text { is relatively compact in } L_{l o c}^{p}\left(\mathbb{R}^{M}\right) \text {. }
$$

Therefore, from Theorem 2.35, we have that $\left\{f_{t} \mid t \in \mathbb{R}\right\}$ is relatively compact in $\mathfrak{L C}_{p}\left(\mathbb{R}^{M}\right)$, and thus $\operatorname{Hull}_{\left(\mathfrak{L C}_{p}\left(\mathbb{R}^{M}\right), \mathcal{T}\right)}(f)$ is compact.

\subsubsection{Weak Topologies}

The problem of compactness in $\mathfrak{L} \mathfrak{C}\left(\mathbb{R}^{M}\right)$ with respect to a weak topology has been investigated in [3] and [4]. In order to recall the results contained in such papers, let us introduce the notion of uniformly integrable $m$-bounds in $L_{l o c}^{1}$ for a Carathéodory function.

Definition 2.37 (Uniformly integrable $m$-bounds in $L_{\text {loc }}^{1}$ ). A set $E \subset \mathfrak{C}\left(\mathbb{R}^{M}\right)$ has uniformly integrable $m$-bounds in $L_{l o c}^{1}$, if for any $j \in \mathbb{N}$ there exists a set $S^{j} \subset L_{l o c}^{1}$ of $m$-bounds of the functions of $E$ on $B_{j}$, such that for every $r>0$ one has that the set $\left\{\left.m\right|_{[-r, r]}:[-r, r] \rightarrow \mathbb{R}^{+} \mid m(\cdot) \in S^{j}\right\}$ is uniformly integrable in $[-r, r]$, i.e. for any $r>0$ and $\varepsilon>0$ there exists a $\delta=\delta(r, \varepsilon)>0$ such that

$$
\forall A \subset[-r, r]: \operatorname{meas}_{\mathbb{R}}(A)<\delta \Rightarrow \sup _{m \in S^{j}} \int_{A} m(t) d t<\varepsilon .
$$

We say that a function $f \in \mathfrak{C}\left(\mathbb{R}^{M}\right)$ has uniformly integrable $m$-bounds in $L_{\text {loc }}^{1}$ if the set of the time translation of $f$ namely $\left\{f_{t} \mid t \in \mathbb{R}\right\}$ has uniformly integrable $m$-bounds in $L_{l o c}^{1}$.

Remark 2.38. Notice that the uniform integrability of the $m$-bounds in $L_{l o c}^{1}$ implies the $L_{l o c}^{1}$-equicontinuity of the $m$-bounds. Moreover, as we have seen in Remark 2.18, if $p>1$, then the $L_{l o c}^{p}$-boundedness implies the $L_{l o c}^{1}$-equicontinuity. However, something a little bit stronger is true. Indeed, as an easy application of Hölder inequality, one has that, for $p>1$, the $L_{l o c}^{p}$-boundedness implies the uniform integrability of the $m$-bounds in $L_{l o c}^{1}$ (and thus also the $L_{l o c}^{1}$-equicontinuity).

In [3] one can find a result of compactness for subsets in $\mathfrak{L} \mathfrak{C}\left(\mathbb{R}^{M}\right)$ with uniformly integrable $m$-bounds in $L_{l o c}^{1}$ and $L_{l o c}^{1}$-bounded $l$-bounds. In particular, the compactness is achieved with respect to the topology (see Proposition 2.4 in the same reference) which is characterized as follows: a sequence $\left(f_{n}\right)_{n \in \mathbb{N}}$ in $\mathfrak{L} \mathfrak{C}\left(\mathbb{R}^{M}\right)$ converges to some $f \in \mathfrak{L} \mathfrak{C}\left(\mathbb{R}^{M}\right)$ if for every $x \in \mathbb{R}^{N}$ and every $t \in \mathbb{R}$ one has

$$
\lim _{n \rightarrow \infty} \int_{0}^{t} f_{n}(s, x) d s=\int_{0}^{t} f(s, x) d s .
$$

However, such a topology is stronger than $\sigma_{D}$ and weaker than $\sigma_{\Theta}$ (both presented in Section 2.2) for any $D$ dense and numerable subset of $\mathbb{R}^{N}$, and for any $\Theta$ 
suitable set of moduli of continuity. Therefore, from the proof of Theorem 2.33, one can easily deduce that the topology characterized in (2.39) coincides with any $\sigma_{D}$ and any $\sigma_{\Theta}$ on any subset of $\mathfrak{L} \mathfrak{C}\left(\mathbb{R}^{M}\right)$ with $L_{l o c}^{1}$-bounded $l$-bounds and $L_{l o c^{-}}^{1}$ equicontinuous $m$-bounds. For the sake of completeness, we include a proof of such a result now updated to any of the weak topologies presented in Section 2.2.

Theorem 2.39. Let $E$ be a subset of $\mathfrak{L} \mathfrak{C}\left(\mathbb{R}^{M}\right)$ with uniformly integrable $m$ bounds in $L_{l o c}^{1}$ and $L_{l o c}^{1}$-bounded l-bounds. Then, $\operatorname{cls}_{\left(\mathfrak{L} \mathfrak{C}\left(\mathbb{R}^{M}\right), \sigma\right)}(E)$ is compact in $\left(\mathfrak{L} \mathfrak{C}\left(\mathbb{R}^{M}\right), \sigma\right)$, where $\sigma$ is either $\sigma_{D}, \sigma_{\Theta}$ or the topology characterized in (2.39).

Proof. As a consequence of Theorem 2.33 we have that all the cited topologies coincide on $E$ and thus we will use the topology $\sigma_{D}$ for our reasoning. Moreover, in order to simplify the notation, let us write $\bar{E}$ in place of $\operatorname{cls}_{\left(\mathfrak{L C}\left(\mathbb{R}^{M}\right), \sigma_{D}\right)}(E)$. Consider a sequence $\left(f_{n}\right)_{n \in \mathbb{N}}$ in $E$. For each $x \in D$ and $r>0$, the sequence $\left(f_{n}(\cdot, x)\right)_{n \in \mathbb{N}}$ of functions with domain in $[-r, r]$ is dominated by the uniformly integrable sequence $\left(m_{f_{n}}^{j}(\cdot)\right)_{n \in \mathbb{N}}$, where $j \in \mathbb{N}$ is chosen so that $|x| \leq j$. Therefore, from Corollary IV.8.11 in [24], one has that the sequence $\left(f_{n}(\cdot, x)\right)_{n \in \mathbb{N}}$ converges, up to a subsequence, to some $f(\cdot, x) \in L^{1}\left([-r, r], \mathbb{R}^{M}\right)$ for the weak topology $\sigma\left(L^{1}\left([-r, r], \mathbb{R}^{M}\right), L^{\infty}\left([-r, r], \mathbb{R}^{M}\right)\right)$ that is, for any $\phi \in L^{\infty}\left([-r, r], \mathbb{R}^{M}\right)$ one has

$$
\int_{-r}^{r} f_{n}(t, x) \phi(t) d t \stackrel{n \rightarrow \infty}{\longrightarrow} \int_{-r}^{r} f(t, x) \phi(t) d t .
$$

Notice that for each $x \in D$ and subsequently enlarging the interval $[-r, r]$, one can extend $f(\cdot, x)$ to the whole $\mathbb{R}$ and thus obtain $f(\cdot, x) \in L_{l o c}^{1}\left(\mathbb{R}^{M}\right)$. By a standard diagonal process, one can find a subsequence, that we keep denoting with the same indexes, such that $\left(f_{n}(\cdot, x)\right)_{n \in \mathbb{N}}$ converges weakly to $f(\cdot, x)$ for all $x \in D$ and in any interval $I \subset \mathbb{R}$ and thus such that

$$
\int_{I} f_{n}(t, x) d t \stackrel{n \rightarrow \infty}{\longrightarrow} \int_{I} f(t, x) d t \quad \forall x \in D \cap B_{j}
$$

Moreover, by assumption the set $\left\{l_{n}^{j}(\cdot) \mid n \in \mathbb{N}\right\}$ is bounded in $L_{l o c}^{1}$ and thus, reasoning as in the proof of Propositions 2.26, we obtain a function $l^{j}(\cdot) \in L_{l o c}^{1}$ such that for any $x, y \in D \cap B_{j}$ the following inequality holds

$$
|f(t, x)-f(t, y)| \leq l^{j}(t)|x-y| \quad \text { for a.e. } t \in \mathbb{R} .
$$

A continuous extension of $f$ to the entire ball $B_{j}$ is given by

$$
f(t, x)=\lim _{n \rightarrow \infty} f\left(t, x_{n}\right) \quad \text { whenever } x \in B_{j},\left(x_{n}\right)_{n \in \mathbb{N}} \text { in } D_{j} \text {, and } x_{n} \rightarrow x .
$$

The definition is well-posed; indeed if $\left(x_{n}\right)_{n \in \mathbb{N}}$ and $\left(y_{n}\right)_{n \in \mathbb{N}}$ are sequences in $D_{j}$ such that $x_{n} \rightarrow x$ and $y_{n} \rightarrow x$, then

$$
\left|f\left(t, x_{n}\right)-f\left(t, y_{n}\right)\right| \leq l^{j}(t)\left|x_{n}-y_{n}\right| \quad \text { for a.e. } t \in \mathbb{R}
$$


and the right-hand side goes to zero as $n \rightarrow \infty$, for almost every $t \in \mathbb{R}$. It is straightforward to prove that $l^{j}(\cdot)$ keeps being a Lipschitz coefficient for the $f$ defined on the whole ball $B_{j}$.

Finally, reasoning as in the last part of the proof of Theorem 2.35, one can prove that $f$ satisfies $(\mathbf{C} 1)$ and $(\mathbf{C} 2)$. As a consequence one has that $\left(f_{n}\right)_{n \in \mathbb{N}}$ converges to $f$ in $\left(\mathfrak{L} \mathfrak{C}\left(\mathbb{R}^{M}\right), \sigma_{D}\right)$, which in turn implies $f \in \bar{E}$, and $\bar{E}$ is compact in $\left(\mathfrak{L} \mathfrak{C}\left(\mathbb{R}^{M}\right), \sigma_{D}\right)$.

One may pose the question whether the uniform integrability of the $m$-bounds in $L_{l o c}^{1}$ is the weakest property which still allows to obtain the compactness of subsets of $\mathfrak{L} \mathfrak{C}\left(\mathbb{R}^{M}\right)$. A thorough look at the proof should make clear why the $L_{l o c}^{1}$-equicontinuity of the $m$-bounds is not sufficient. Roughly speaking, taken a sequence of absolutely continuous measures with $L_{l o c}^{1}$-equicontinuous densities, and assuming that such sequence converges in the vague topology to a measure $\mu$, we are not able to tell whether $\mu$ is absolutely continuous or not. In other words, we might end up with a limit problem which can not be formulated in terms of an ordinary differential equation. As a matter of fact, it is shown in [4] that a subset of $\mathfrak{L} \mathfrak{C}\left(\mathbb{R}^{M}\right)$ with $L_{\text {loc }}^{1}$-equicontinuous $m$-bounds and $L_{l o c}^{1}$-bounded $l$-bounds is precompact with respect to the topology characterized in (2.39) (and thus also with respect to any $\sigma_{D}$ and any $\sigma_{\Theta}$ as a consequence of Theorem 2.33) and, by identifying functions in $\mathfrak{L} \mathfrak{C}\left(\mathbb{R}^{M}\right)$ with their respective differential equations, it admits a compactification in the space of Kurzweil equations. Such topic, although interesting and worthwhile, falls beyond the scope of this work, and thus will not be covered. We recommend [4] for further details.

\subsection{Continuity of the time translations}

This section contains the first step to build a continuous skew product flow for differential equations of Carathéodory type. In particular, we present two theorems providing sufficient conditions to obtain continuity of the base flow, i.e. continuity of the time-translations (see Definition 1.9), in $\left(\Theta \mathfrak{C}_{p}\left(\mathbb{R}^{M}\right), \mathcal{T}_{\Theta}\right)$ and $\left(\mathfrak{W} \Theta \mathfrak{C}\left(\mathbb{R}^{M}\right), \sigma_{\Theta}\right)$, respectively.

Theorem 2.40. Let $\Theta$ be a suitable set of moduli of continuity as in Definition 2.3. The map

$$
\mathbb{R} \times \Theta \mathfrak{C}_{p}\left(\mathbb{R}^{M}\right) \rightarrow \Theta \mathfrak{C}_{p}\left(\mathbb{R}^{M}\right), \quad(t, f) \mapsto f_{t},
$$

defines a continuous flow on $\left(\Theta \mathfrak{C}_{p}\left(\mathbb{R}^{M}\right), \mathcal{T}_{\Theta}\right)$.

Proof. We separately deal with the continuity with respect to $f$ and with respect to $t$, and eventually gather them together. Let $\left(f_{n}\right)_{n \in \mathbb{N}}$ be a sequence in $\Theta \mathfrak{C}_{p}\left(\mathbb{R}^{M}\right)$ converging to some $f$ in $\left(\Theta \mathfrak{C}_{p}\left(\mathbb{R}^{M}\right), \mathcal{T}_{\Theta}\right)$. We prove that

$$
\left(f_{n}\right)_{t} \stackrel{n \rightarrow \infty}{\longrightarrow} f_{t}, \quad \text { in }\left(\Theta \mathfrak{C}_{p}\left(\mathbb{R}^{M}\right), \mathcal{T}_{\Theta}\right),
$$


uniformly for $t$ in a compact interval. Consider $I=\left[p_{1}, p_{2}\right]$ and $J=\left[q_{1}, q_{2}\right]$ such that $p_{1}, p_{2}, q_{1}, q_{2} \in \mathbb{Q}, 0 \in J$ and fix $t \in J$. Moreover, for any $j \in \mathbb{N}$ consider $\mathcal{K}_{j}^{I}$ and $\mathcal{K}_{j}^{I+J}$ as in Definition 2.4. Notice that $x(\cdot) \in \mathcal{K}_{j}^{I}$ implies $x(\cdot-t) \in \mathcal{K}_{j}^{I+J}$ up to a suitable extension by constants of the function $x(\cdot-t)$ in $I+J$. Then

$$
\begin{aligned}
\lim _{n \rightarrow \infty} \sup _{x(\cdot) \in \mathcal{K}_{j}^{I}} \int_{I}\left|\left(f_{n}\right)_{t}(s, x(s))-f_{t}(s, x(s))\right|^{p} d s & \\
\quad & \lim _{n \rightarrow \infty} \sup _{x(\cdot) \in \mathcal{K}_{j}^{I}} \int_{I+t}\left|f_{n}(r, x(r-t))-f(r, x(r-t))\right|^{p} d r \\
& \leq \lim _{n \rightarrow \infty} \sup _{x(\cdot) \in \mathcal{K}_{j}^{I+J}} \int_{I+J}\left|f_{n}(r, x(r))-f(r, x(r))\right|^{p} d r=0 .
\end{aligned}
$$

Next, we prove the continuity with respect to the first variable; in other words, the map $t \mapsto f_{t}$ of $\mathbb{R}$ into $\left(\Theta \mathfrak{C}_{p}\left(\mathbb{R}^{M}\right), \mathcal{T}_{\Theta}\right)$ is continuous. Consider $f \in \Theta \mathfrak{C}_{p}\left(\mathbb{R}^{M}\right)$, $I=[a, b]$ where $a, b \in \mathbb{Q}$ and $t \in \mathbb{R}$ fixed. We aim to prove that for any compact set $\mathcal{K}_{j}^{I}$, as in Definition 2.4, we have that

$$
\lim _{\tau \rightarrow 0} \sup _{x(\cdot) \in \mathcal{K}_{j}^{I}} \int_{I}\left|f_{t+\tau}(s, x(s))-f_{t}(s, x(s))\right|^{p} d s=0 .
$$

Firstly, let us fix $x(\cdot) \in \mathcal{K}_{j}^{I}$ and prove that if $\tau_{n} \rightarrow 0$ as $n \rightarrow \infty$ then

$$
\lim _{n \rightarrow \infty} \int_{I}\left|f_{t+\tau_{n}}(s, x(s))-f_{t}(s, x(s))\right|^{p} d s=0
$$

Notice that $f_{t}(\cdot, x(\cdot)) \in L^{p}\left(I, \mathbb{R}^{M}\right)$ and consider the operator

$$
T_{\tau}: L^{p}\left(I, \mathbb{R}^{M}\right) \rightarrow L^{p}\left(\mathbb{R}, \mathbb{R}^{M}\right), \quad \text { such that } g(\cdot) \mapsto T_{\tau} g(\cdot)
$$

where $T_{\tau} g(\cdot)$ is defined by

$$
T_{\tau} g(s)=\left\{\begin{array}{cl}
g(s+\tau), & \text { if } s+\tau \in I \\
0, & \text { otherwise }
\end{array}\right.
$$

By the continuity of translations in $L^{p}(I)$, see Castillo and Rafeiro [17, Theorem 3.58], we have that, if $\left|\tau_{n}\right| \rightarrow 0$ as $n \rightarrow \infty$, then for a given $\varepsilon>0$ there exists $\delta>0$ such that

$$
\sup _{\left|\tau_{n}\right|<\delta}\left\|T_{\tau_{n}} f_{t}(\cdot, x(\cdot))-f_{t}(\cdot, x(\cdot))\right\|_{p} \leq \varepsilon .
$$

Now, for any $n \in \mathbb{N}$, define $a_{n}=\max \left\{a, a-\tau_{n}\right\}$ and $b_{n}=\min \left\{b, b-\tau_{n}\right\}$, and consider $n_{0} \in \mathbb{N}$ so that for any $n>n_{0}$ we have $\left|\tau_{n}\right|<\delta$. Therefore, for any 
$n>n_{0}$ the following chain of inequalities holds true

$$
\begin{aligned}
&\left\|f_{t+\tau_{n}}(\cdot, x(\cdot))-f_{t}(\cdot, x(\cdot))\right\|_{p} \\
& \leq\left\|T_{\tau_{n}} f_{t}(\cdot, x(\cdot))-f_{t}(\cdot, x(\cdot))\right\|_{p}+\left\|f_{t+\tau_{n}}(\cdot, x(\cdot))-T_{\tau_{n}} f_{t}(\cdot, x(\cdot))\right\|_{p} \\
& \leq \varepsilon+\left\|f_{t+\tau_{n}}(\cdot, x(\cdot))-T_{\tau_{n}} f_{t}(\cdot, x(\cdot))\right\|_{p} \\
& \leq \varepsilon+\left[\int_{a_{n}}^{b_{n}}\left|f_{t}\left(s+\tau_{n}, x(s)\right)-f_{t}\left(s+\tau_{n}, x\left(s+\tau_{n}\right)\right)\right|^{p} d s\right]^{1 / p} \\
&+\left[\int_{a}^{a_{n}}\left|f_{t}\left(s+\tau_{n}, x(s)\right)\right|^{p} d s\right]^{1 / p}+\left[\int_{b_{n}}^{b}\left|f_{t}\left(s+\tau_{n}, x(s)\right)\right|^{p} d s\right]^{1 / p} \\
& \leq \varepsilon+\left[\int_{a_{n}+\tau_{n}}^{b_{n}+\tau_{n}}\left|f_{t}\left(u, x\left(u-\tau_{n}\right)\right)-f_{t}(u, x(u))\right|^{p} d u\right]^{1 / p} \\
&+ {\left[\int_{a+\tau_{n}}^{a_{n}+\tau_{n}}\left|f_{t}\left(u, x\left(u-\tau_{n}\right)\right)\right|^{p} d u\right]^{1 / p} } \\
&+ {\left[\int_{b_{n}+\tau_{n}}^{b+\tau_{n}}\left|f_{t}\left(u, x\left(u-\tau_{n}\right)\right)\right|^{p} d u\right]^{1 / p}=\varepsilon+I_{1}+I_{2}+I_{3} }
\end{aligned}
$$

As regards $I_{1}$, notice that, up to extending the functions $x(\cdot)$ and $\left(x\left(\cdot-\tau_{n}\right)\right)_{n \in \mathbb{N}}$ by constants to an interval $J$ containing $I+[-\delta, \delta]$ we have that

$$
I_{1} \leq\left[\int_{J}\left|f_{t}\left(u, x\left(u-\tau_{n}\right)\right)-f_{t}(u, x(u))\right|^{p} d u\right]^{1 / p},
$$

and the integral on the right-hand side goes to zero as $n \rightarrow \infty$, due to the fact that $f \in \Theta \mathfrak{C}_{p}\left(\mathbb{R}^{M}\right)$ and $\left\|x\left(\cdot-\tau_{n}\right)-x(\cdot)\right\|_{\infty} \rightarrow 0$ in $J$ as $n \rightarrow \infty$. As regards $I_{2}$, let $m^{j}$ be an $m$-bound of $f$ on $B_{j}$ and notice that the following chain of inequalities holds

$$
I_{2} \leq\left[\int_{a-\left|\tau_{n}\right|}^{a}\left|f_{t}\left(u, x\left(u-\tau_{n}\right)\right)\right|^{p} d u\right]^{1 / p} \leq\left[\int_{a-\left|\tau_{n}\right|}^{a}\left(m_{t}^{j}(u)\right)^{p} d u\right]^{1 / p},
$$

and the integral on the right-hand side of equation (2.43) goes to zero as $n \rightarrow \infty$, thanks to the absolute continuity of the Lebesgue integral. Similar reasonings apply to $I_{3}$. Therefore, for any fixed $t \in \mathbb{R}$ and $x(\cdot) \in \mathcal{K}_{j}^{I}$ we obtain the limit in (2.42). Next we check that such a convergence is uniform in $\mathcal{K}_{j}^{I}$. Otherwise there would exist an $\varepsilon>0$, a sequence $\left(x_{n}(\cdot)\right)_{n \in \mathbb{N}}$ in $\mathcal{K}_{j}^{I}$, and a sequence $\left(\tau_{n}\right)_{n \in \mathbb{N}}$ in $\mathbb{R}$ converging to 0 , such that

$$
\left[\int_{I}\left|f_{t+\tau_{n}}\left(s, x_{n}(s)\right)-f_{t}\left(s, x_{n}(s)\right)\right|^{p} d s\right]^{1 / p}>\varepsilon, \quad \forall n \in \mathbb{N} .
$$

However, being $\mathcal{K}_{j}^{I}$ compact, there exists a subsequence of $\left(x_{n}(\cdot)\right)_{n \in \mathbb{N}}$, which we keep denoting with the same indexes, converging uniformly in $I$ to some $x(\cdot) \in \mathcal{K}_{j}^{I}$ as $n \rightarrow \infty$. From (2.42), there exists $n_{0} \in \mathbb{N}$ such that, if $n>n_{0}$, then

$$
\left\|f_{t+\tau_{n}}(\cdot, x(\cdot))-f_{t}(\cdot, x(\cdot))\right\|_{p}<\frac{\varepsilon}{4} .
$$


Moreover, since $f_{t} \in \Theta \mathfrak{C}_{p}\left(\mathbb{R}^{M}\right)$ and $\left(x_{n}(\cdot)\right)_{n \in \mathbb{N}}$ converges uniformly to $x(\cdot)$, there exists $n_{1} \in \mathbb{N}$ such that, if $n>n_{1}$, then

$$
\left\|f_{t}(\cdot, x(\cdot))-f_{t}\left(\cdot, x_{n}(\cdot)\right)\right\|_{p}<\frac{\varepsilon}{4} .
$$

Then, for $n>\max \left\{n_{0}, n_{1}\right\}$, we have that

$$
\begin{aligned}
\varepsilon & <\left\|f_{t+\tau_{n}}\left(\cdot, x_{n}(\cdot)\right)-f_{t}\left(\cdot, x_{n}(\cdot)\right)\right\|_{p} \\
& \leq\left\|f_{t+\tau_{n}}\left(\cdot, x_{n}(\cdot)\right)-f_{t+\tau_{n}}(\cdot, x(\cdot))\right\|_{p}+\left\|f_{t+\tau_{n}}(\cdot, x(\cdot))-f_{t}(\cdot, x(\cdot))\right\|_{p} \\
& \quad+\left\|f_{t}(\cdot, x(\cdot))-f_{t}\left(\cdot, x_{n}(\cdot)\right)\right\|_{p} \\
& =A_{1}+\varepsilon / 4+\varepsilon / 4 .
\end{aligned}
$$

Finally, notice that

$$
\begin{aligned}
A_{1} & =\left[\int_{I+\tau_{n}}\left|f_{t}\left(u, x_{n}\left(u-\tau_{n}\right)\right)-f_{t}\left(u, x\left(u-\tau_{n}\right)\right)\right|^{p} d u\right]^{1 / p} \\
& \leq\left[\int_{J}\left|f_{t}\left(u, x_{n}\left(u-\tau_{n}\right)\right)-f_{t}\left(u, x\left(u-\tau_{n}\right)\right)\right|^{p} d u\right]^{1 / p}<\frac{\varepsilon}{4},
\end{aligned}
$$

for $n$ greater than some $n_{2} \in \mathbb{N}$ since, once again, $f_{t} \in \Theta \mathfrak{C}_{p}\left(\mathbb{R}^{M}\right)$ and $\left(x_{n}(\cdot)\right)_{n \in \mathbb{N}}$ converges uniformly to $x(\cdot)$. Gathering (2.46), (2.44), (2.45) and (2.47) we get a contradiction, which implies the uniform limit in (2.41).

In order to conclude the proof, we check the joint continuity. Consider $\left(f_{n}\right)_{n \in \mathbb{N}} \subset \Theta \mathfrak{C}_{p}\left(\mathbb{R}^{M}\right)$ converging to some $f$ in $\left(\Theta \mathfrak{C}_{p}\left(\mathbb{R}^{M}\right), \mathcal{T}_{\Theta}\right)$ and $\left(t_{n}\right)_{n \in \mathbb{N}} \subset \mathbb{R}$ converging to some $t \in \mathbb{R}$. Fixed $j \in \mathbb{N}, I=\left[q_{1}, q_{2}\right]$, with $q_{1}, q_{2} \in \mathbb{Q}$, and $\mathcal{K}_{j}^{I}$ as in Definition 2.4, recalling that the limit in (2.40) is uniform for $t$ in compact sets, we have that

$$
\begin{aligned}
\lim _{n \rightarrow \infty} \sup _{x(\cdot) \in \mathcal{K}_{j}^{I}} & {\left[\int_{I}\left|\left(f_{n}\right)_{t_{n}}(s, x(s))-f_{t}(s, x(s))\right|^{p} d s\right]^{1 / p} } \\
\leq & \lim _{n \rightarrow \infty} \sup _{x(\cdot) \in \mathcal{K}_{j}^{I}}\left[\int_{I}\left|\left(f_{n}\right)_{t_{n}}(s, x(s))-f_{t_{n}}(s, x(s))\right|^{p} d s\right]^{1 / p} \\
& \quad+\lim _{n \rightarrow \infty} \sup _{x(\cdot) \in \mathcal{K}_{j}^{I}}\left[\int_{I}\left|f_{t_{n}}(s, x(s))-f_{t}(s, x(s))\right|^{p} d s\right]^{1 / p}=0,
\end{aligned}
$$

which ends the proof.

Remark 2.41. The continuity of the time translation map in $\left(\mathfrak{S C}_{p}\left(\mathbb{R}^{M}\right), \mathcal{T}_{D}\right)$ can be easily proved using the same arguments of the proof of Theorem 2.40. Therefore, the proof is omitted. Furthermore, the continuity in $\left(\mathfrak{S C}_{p}\left(\mathbb{R}^{M}\right), \mathcal{T}_{B}\right)$ is stated in [53, p.53] and the proof can be derived by the one given in [41, Lemma II.1 p.24] for $\left(\mathfrak{L} \mathfrak{C}_{p}\left(\mathbb{R}^{M}\right), \mathcal{T}_{B}\right)$. 
As a corollary of what we have seen so far in the section, we deduce a first theorem of continuity of the translations on the hull of a Carathéodory function.

Corollary 2.42. Let $(E, \mathcal{T})$ be any of the following topological spaces (or any topological subspace of them):

$$
\left(\Theta \mathfrak{C}\left(\mathbb{R}^{M}\right), \mathcal{T}_{\Theta}\right), \quad\left(\mathfrak{S C}_{p}\left(\mathbb{R}^{M}\right), \mathcal{T}_{D}\right) \quad \text { and } \quad\left(\mathfrak{S C}_{p}\left(\mathbb{R}^{M}\right), \mathcal{T}_{B}\right)
$$

Then, considered $f \in E$, the map

$$
\mathbb{R} \times \operatorname{Hull}_{(E, \mathcal{T})}(f) \rightarrow \operatorname{Hull}_{(E, \mathcal{T})}(f), \quad(t, g) \mapsto g_{t},
$$

defines a continuous flow on $\operatorname{Hull}_{(E, \mathcal{T})}(f)$.

Next, the continuity of the time-translations in the space $\left(\mathfrak{W} \Theta \mathfrak{C}\left(\mathbb{R}^{M}\right), \sigma_{\Theta}\right)$ is proved. Notice that a key difference arises with respect to Theorem 2.40. Indeed, while trying to accomplish the same result in a larger space and with a weaker topology, we are forced to assume $L_{l o c}^{1}$-equicontinuity of the $m$-bounds.

Theorem 2.43. Let $\Theta$ be a suitable set of moduli of continuity as in Definition 2.3 and consider the space $\left(\mathfrak{W} \Theta \mathfrak{C}\left(\mathbb{R}^{M}\right), \sigma_{\Theta}\right)$. If $E \subset \mathfrak{W} \Theta \mathfrak{C}\left(\mathbb{R}^{M}\right)$ admit $L_{\text {loc }}^{1}$ equicontinuous $m$-bounds, then, denoted by $\bar{E}=\operatorname{cls}_{\left(\mathfrak{W} \Theta \mathfrak{C}\left(\mathbb{R}^{M}\right), \sigma_{\Theta}\right)}(E)$, one has that the map

$$
\mathbb{R} \times \bar{E} \rightarrow \mathfrak{W} \Theta \mathfrak{C}\left(\mathbb{R}^{M}\right), \quad(t, f) \mapsto f_{t},
$$

is continuous.

Proof. Firstly, notice that the map is well-defined thanks to Lemma 2.28. Let $\left(f_{n}\right)_{n \in \mathbb{N}}$ be a sequence in $E$ converging to $f$ in $\left(\mathfrak{W} \Theta \mathfrak{C}\left(\mathbb{R}^{M}\right), \sigma_{\Theta}\right)$ and $\left(t_{n}\right)_{n \in \mathbb{N}}$ a sequence in $\mathbb{R}$ converging to $t \in \mathbb{R}$. We want to prove that for every $I=\left[q_{1}, q_{2}\right]$, $q_{1}, q_{2} \in \mathbb{Q}$, and every $j \in \mathbb{N}$ one has that

$$
\lim _{n \rightarrow \infty} \sup _{x(\cdot) \in \mathcal{K}_{j}^{I}}\left|\int_{I}\left[f_{n}\left(t_{n}+s, x(s)\right)-f(t+s, x(s))\right] d s\right|=0,
$$

where $\mathcal{K}_{j}^{I}$ is as in Definition 2.4. Let us fix $\varepsilon>0, j \in \mathbb{N}$, and $I=\left[q_{1}, q_{2}\right]$, $q_{1}, q_{2} \in \mathbb{Q}$ and consider an interval $\left[r_{1}, r_{2}\right]$ such that, for every $n \in \mathbb{N}$, one has $\left[q_{1}+t_{n}, q_{2}+t_{n}\right] \subset\left[r_{1}, r_{2}\right]$. Since $E$ admits $L_{l o c}^{1}$-equicontinuous $m$-bounds, and thanks to Proposition 2.29, one has that there exists $\delta>0$ such that

$$
\sup _{g \in \bar{E}} \int_{\tau_{1}}^{\tau_{2}} m_{g}^{j}(u) d u<\varepsilon / 6
$$

whenever $\tau_{1}, \tau_{2} \in\left[r_{1}, r_{2}\right]$ and $0 \leq \tau_{2}-\tau_{1}<\delta$. Consider $p_{1}(t), p_{2}(t) \in \mathbb{Q}$ such that $q_{1}+t<p_{1}(t)<p_{2}(t)<q_{2}+t$ and

$$
p_{1}(t)-q_{1}-t<\delta \quad \text { and } \quad q_{2}+t-p_{2}(t)<\delta \text {. }
$$


Notice also that, since $t_{n} \rightarrow t$, then there exists $n_{0} \in \mathbb{N}$ such that for every $n>n_{0}$ one has that $q_{1}+t_{n}<p_{1}(t)<p_{2}(t)<q_{2}+t_{n}$ and

$$
p_{1}(t)-q_{1}-t_{n}<\delta \text { and } q_{2}+t_{n}-p_{2}(t)<\delta .
$$

Then, for every $n>n_{0}$ one has that

$$
\begin{aligned}
\sup _{x(\cdot) \in \mathcal{K}_{j}^{I}} \mid & \int_{I}\left[f_{n}\left(t_{n}+s, x(s)\right)-f(t+s, x(s))\right] d s \mid \\
= & \sup _{x(\cdot) \in \mathcal{K}_{j}^{I}}\left|\int_{q_{1}+t_{n}}^{q_{2}+t_{n}} f_{n}\left(u, x\left(u-t_{n}\right)\right) d u-\int_{q_{1}+t}^{q_{2}+t} f(u, x(u-t)) d u\right| \\
\leq & \sup _{x(\cdot) \in \mathcal{K}_{j}^{I}}\left|\int_{p_{1}(t)}^{p_{2}(t)}\left[f_{n}\left(u, x\left(u-t_{n}\right)\right)-f(u, x(u-t))\right] d u\right|+\frac{4 \varepsilon}{6} \\
\leq & \sup _{x(\cdot) \in \mathcal{K}_{j}^{I}}\left|\int_{p_{1}(t)}^{p_{2}(t)}\left[f_{n}\left(u, x\left(u-t_{n}\right)\right)-f\left(u, x\left(u-t_{n}\right)\right)\right] d u\right|+ \\
& \quad+\sup _{x(\cdot) \in \mathcal{K}_{j}^{I}}\left|\int_{p_{1}(t)}^{p_{2}(t)}\left[f\left(u, x\left(u-t_{n}\right)\right)-f(u, x(u-t))\right] d u\right|+\frac{2 \varepsilon}{3} \\
= & P_{n}+R_{n}+\frac{2 \varepsilon}{3} .
\end{aligned}
$$

If we take an interval $J$ with rational extremes so that $I \cup\left[p_{1}(t), p_{2}(t)\right] \subset J$, then, up to a suitable extension by constants to $J$, the functions $y_{n}(\cdot)=x\left(\cdot-t_{n}\right)$ belong to $\mathcal{K}_{j}^{J}$ and we deduce that

$$
\lim _{n \rightarrow \infty} P_{n} \leq \lim _{n \rightarrow \infty} \sup _{y(\cdot) \in \mathcal{K}_{j}^{J}}\left|\int_{p_{1}(t)}^{p_{2}(t)}\left[f_{n}(u, y(u))-f(u, y(u))\right] d u\right|=0
$$

because $\left(f_{n}\right)_{n \in \mathbb{N}}$ converges to $f$ in $\left(\mathfrak{W} \Theta \mathfrak{C}\left(\mathbb{R}^{M}\right), \sigma_{\Theta}\right)$ and Lemma 2.14(ii) can be applied.

Analogously, recalling that $f \in \mathfrak{W} \Theta \mathfrak{C}\left(\mathbb{R}^{M}\right)$ satisfies (W), from Lemma 2.14(i) we deduce that $\lim _{n \rightarrow \infty} R_{n}=0$. Therefore, gathering together the results on $P_{n}$ and $R_{n}$, and by the arbitrariness of $\varepsilon$, from (2.49) we obtain (2.48) which ends the proof.

Remark 2.44. The continuity of the time translation map in $\left(\mathfrak{S C}\left(\mathbb{R}^{M}\right), \sigma_{D}\right)$ can be easily deduced from the proof of the previous theorem.

As a corollary of the previous theorem, one has that the map defined by the time-translations is a continuous flow on the hull of a function in $\mathfrak{W} \Theta \mathfrak{C}\left(\mathbb{R}^{M}\right)$ with $L_{l o c}^{1}$-equicontinuous $m$-bounds.

Corollary 2.45. Let $f \in \mathfrak{W} \Theta \mathfrak{C}\left(\mathbb{R}^{M}\right)$ admit $L_{\text {loc }}^{1}$-equicontinuous m-bounds. Then, the map

$$
\mathbb{R} \times \operatorname{Hull}_{\left(\mathfrak{W} \Theta \mathfrak{C}\left(\mathbb{R}^{M}\right), \sigma_{\Theta}\right)}(f) \rightarrow \operatorname{Hull}_{\left(\mathfrak{W} \Theta \mathfrak{C}\left(\mathbb{R}^{M}\right), \sigma_{\Theta}\right)}(f), \quad(t, g) \mapsto g_{t},
$$

defines a continuous flow on $\operatorname{Hull}_{\left(\mathfrak{W} \Theta \mathfrak{C}\left(\mathbb{R}^{M}\right), \sigma_{\Theta}\right)}(f)$. 
Proof. The result is a direct consequence of Theorem 2.43. In particular, fixed $t \in \mathbb{R}$ and a sequence $\left(f_{\tau_{n}}\right)_{n \in \mathbb{N}}$ of time translations of $f$ converging to $g \in$ $\operatorname{Hull}_{\left(\mathfrak{W} \Theta \mathfrak{C}\left(\mathbb{R}^{M}\right), \sigma_{\Theta}\right)}(f)$, due to $(2.48)$, one has

$$
\lim _{n \rightarrow \infty} \sup _{x(\cdot) \in \mathcal{K}_{j}^{I}}\left|\int_{I}\left[f_{\tau_{n}}(t+s, x(s))-g(t+s, x(s))\right] d s\right|=0,
$$

and thus $g_{t} \in \operatorname{Hull}_{\left(\mathfrak{W} \Theta \mathfrak{C}\left(\mathbb{R}^{M}\right), \sigma_{\Theta}\right)}(f)$ for all $t \in \mathbb{R}$, which implies that the map in (2.50) is well-posed. The continuity is then a direct consequence of Theorem 2.43 . 


\section{Chapter 3}

\section{Continuity of the flow for Carathéodory ODEs}

In this chapter, we address the problem of defining a continuous skew-product flow for Carathéodory ordinary differential equations of the type

$$
\dot{x}=f(t, x), \quad x(0)=x_{0},
$$

and Carathéodory systems of triangular type as

$$
\begin{cases}\dot{x}=f(t, x), & x(0)=x_{0}, \\ \dot{y}=F(t, x) y+h(t, x), & y(0)=y_{0},\end{cases}
$$

where the function $f(\cdot, \cdot)$ is Lipschitz Carathéodory, and $F(\cdot, \cdot)$ and $h(\cdot, \cdot)$ are taken either in $\mathfrak{W} \Theta \mathfrak{C}$ or in $\Theta \mathfrak{C}_{p}$, so that the problem is well-posed. Due to the the variety of topologies presented in Section 2.2 and of assumptions on the $m$ bounds and/or $l$-bounds presented in Section 2.3, one may find several results of continuity for the induced skew-product flow.

In Section 3.1 we provide the optimal ones in terms of the strength of the used topologies, and of the assumptions on the initial vector field. As follows, we portray the scheme of assumptions in the three cases (the notation is simplified neglecting the dimension of the image space).

Case 1: $\quad f \in\left(\mathfrak{L C}, \sigma_{\Theta}\right)$ with $L_{\text {loc }}^{1}$-equicontinuous $m$-bounds, $F \in\left(\mathfrak{W} \Theta \mathfrak{C}, \sigma_{\Theta}\right)$ with $L_{l o c}^{1}$-equicontinuous $m$-bounds, $h \in\left(\mathfrak{W} \Theta \mathfrak{C}, \sigma_{\Theta}\right)$ with $L_{l o c}^{1}$-equicontinuous $m$-bounds.

Case 2: $\quad f \in\left(\mathfrak{L} \mathfrak{C}_{p}, \mathcal{T}_{\Theta}\right)$ with $L_{\text {loc }}^{1}$-equicontinuous $m$-bounds, $F \in\left(\Theta \mathfrak{C}_{p}, \mathcal{T}_{\Theta}\right), h \in\left(\Theta \mathfrak{C}_{p}, \mathcal{T}_{\Theta}\right)$.

Case 3: $\quad f \in\left(\mathfrak{L} \mathfrak{C}_{p}, \mathcal{T}_{D}\right)$ with $L_{\text {loc }}^{p}$-bounded l-bounds, $F \in\left(\Theta \mathfrak{C}_{p}, \mathcal{T}_{\Theta}\right), h \in\left(\Theta \mathfrak{C}_{p}, \mathcal{T}_{\Theta}\right)$. 
A trade between the strength of the used topologies and the assumptions on the functions $f, F$, and $h$, arises. With few assumptions on the initial vector fields, it will be necessary to use a stronger topology and vice versa.

The last sections of the chapter deal with applications of the continuity of the skew product flow. Particularly, in Section 3.2 we treat the case in which $f$ is continuously differentiable with respect to $x$ and the second equation in (3.2) is the variational equation of the first one, that is, $F=J_{x} f$ is a strong Carathéodory function and $h=0$. Applying the results contained in Section 3.1 we are able to construct two types of linearized skew-product flows depending on the topology used, either $\mathcal{T}_{\Theta}$ or $\sigma_{\Theta}$. We conclude the section with an example portraying the atypical case in which it is possible (thanks to the continuity of the skew-product flow) to obtain the differentiability of the solutions with respect to initial data for a system which is not continuously differentiable in the variable $x$, and thus, it does not admit the classic variational equation.

In Section 3.3 we show how to propagate the exponential dichotomy of a linearized system through the trajectories of the linearized skew-product flows.

Section 3.4 contains a further theoretical result and two examples. Specifically, thanks to the continuity of the time translations in $\left(\mathfrak{W} \Theta \mathfrak{C}, \sigma_{\Theta}\right)$ given in Theorem 2.43 and thanks to the theorems of continuity of the solutions with respect to the variation of initial data and vector field in Section 3.1, we are able to prove to existence of solutions for differential problem whose vector field is a function in $\mathfrak{W} \Theta \mathfrak{C}$ which is the limit, with respect to the topology $\sigma_{\Theta}$, of a sequence in $\mathfrak{S} \mathfrak{C}$.

Furthermore, we notice that the assumptions used in [25] to obtain stability results for an abstract digitization scheme in control theory, are such that Theorem 2.31 applies, and thus the integral-like topology used in such work coincide with any of the strong topologies considered in Chapter 2. Additionally, we highlight how some of the results contained in such paper can be read in view of the theory developed in this work.

Finally, inspired by [50], where a study of a triangular system composed of a non-autonomous linear compartmental system and an induced mean-age system is accomplished, we construct a triangular system for a Carathéodory non-linear compartmental system and point out how a continuous skew-product flow can be obtained if any of the theorems in Section 3.1 applies.

\subsection{Continuity of the flow}

This section contains several results of continuity for skew-product flows generated by either, a single Carathéodory system with vector field in $\mathfrak{L C}_{p}$ or, by triangular systems composed of a nonlinear system with vector field in $\mathfrak{L} \mathfrak{C}_{p}$ and a linear system with vector field in $\mathfrak{W} \Theta \mathfrak{C}$ or $\Theta \mathfrak{C}_{p}$. The joint role of $m$-bounds and $l$-bounds in proving the continuous variation of ODEs' solutions with respect 
to initial conditions, has been investigated in [3, 4] when weak topologies are involved. However, later on we show how assumptions on the sole $m$-bounds, or the sole $l$-bounds, can still be sufficient to prove the continuous variation of ODEs solutions when weak topologies are used. Furthermore, we show how such topological properties turn out to be useful in order to prove the continuity when the strong topologies are employed and which differences arise. We state the following theorem as a corollary of Theorem 1.2 to set some notation.

Corollary 3.1. Let $\Theta$ be a suitable set of moduli of continuity as in Definition 2.3. For any $f \in \mathfrak{L} \mathfrak{C}, F \in \mathfrak{W} \Theta \mathfrak{C}\left(\mathbb{R}^{N \times N}\right), h \in \mathfrak{W} \Theta \mathfrak{C}$, and $x_{0}, y_{0} \in \mathbb{R}^{N}$, there exists a unique solution of the Cauchy problem

$$
\begin{cases}\dot{x}=f(t, x), & x(0)=x_{0}, \\ \dot{y}=F(t, x) y+h(t, x), & y(0)=y_{0}\end{cases}
$$

which will be denoted by $\left(x\left(\cdot, f, x_{0}\right), y\left(\cdot, f, F, h, x_{0}, y_{0}\right)\right)$, and whose maximal interval of definition coincides with the interval $I_{f, x_{0}}$ provided by Theorem 1.2.

The suitable set of moduli of continuity $\Theta$ used to determine the sets $\mathfrak{W} \Theta \mathfrak{C}$ and/or $\Theta \mathfrak{C}_{p}$, assumes particular relevance when one wants to prove the continuity of the solutions with respect to the variation of initial conditions and thus the existence of a continuous skew-product flow. In the first two theorems of continuity of the flow presented in this section, the set $\Theta$ will be constructed, as follows, using the $m$-bounds of the vector fields defining our initial systems.

Definition 3.2 (Moduli of continuity given by the $m$-bounds). Let $E \subset \mathfrak{L} \mathfrak{C}$ admit $L_{\text {loc }}^{1}$-equicontinuous $m$-bounds. For any $j \in \mathbb{N}$ and for any interval $I=\left[q_{1}, q_{2}\right]$, $q_{1}, q_{2} \in \mathbb{Q}$, define

$$
\theta_{j}^{I}(s):=\sup _{t \in I, f \in E} \int_{t}^{t+s} m_{f}^{j}(u) d u, \quad s \geq 0
$$

where, for any $f \in E$, the function $m_{f}^{j}(\cdot) \in L_{l o c}^{1}$ denotes the optimal $m$-bounds of $f$ on $B_{j}$. Notice that, since $E$ admits $L_{l o c}^{1}$-equicontinuous $m$-bounds, then $\Theta=\left\{\theta_{j}^{I}(\cdot) \mid I=\left[q_{1}, q_{2}\right], q_{1}, q_{2} \in \mathbb{Q}, j \in \mathbb{N}\right\}$ defines a suitable set of moduli of continuity (see Definition 2.3).

Remark 3.3. If $f \in \mathfrak{L} \mathfrak{C}$ has $L_{l o c}^{1}$-equicontinuous $m$-bounds we similarly define for any $j \in \mathbb{N}$,

$$
\theta_{j}(s):=\sup _{t \in \mathbb{R}} \int_{t}^{t+s} m^{j}(u) d u, \quad s \geq 0,
$$

where $m^{j}(\cdot)$ is the optimal $m$-bound for $f$ on $B_{j}$. Here again, notice that, thanks to the $L_{l o c}^{1}$-equicontinuity of the $m$-bounds of $f$, one has that $\Theta=\left\{\theta_{j}(\cdot) \mid j \in \mathbb{N}\right\}$ defines a suitable set of moduli of continuity (see Definition 2.3). 
Theorem 3.4. Consider $E \subset \mathfrak{L} \mathfrak{C}$ with $L_{\text {loc }}^{1}$-equicontinuous $m$-bounds and let $\Theta$ be the countable set of moduli of continuity in Definition 3.2. Additionally, consider $B \subset \mathfrak{W} \Theta \mathfrak{C}\left(\mathbb{R}^{N \times N}\right)$ and $C \subset \mathfrak{W} \Theta \mathfrak{C}$, both with $L_{\text {loc }}^{1}$-equicontinuous m-bounds. With the notation of Theorem 1.2 and Corollary 3.1,

(i) if $\left(f_{n}\right)_{n \in \mathbb{N}}$ in $E$ converges to $f$ in $\left(\mathfrak{L} \mathfrak{C}, \sigma_{\Theta}\right)$ and $\left(x_{0, n}\right)_{n \in \mathbb{N}}$ in $\mathbb{R}^{N}$ converges to $x_{0} \in \mathbb{R}^{N}$, then

$$
x\left(\cdot, f_{n}, x_{0, n}\right) \stackrel{n \rightarrow \infty}{\longrightarrow} x\left(\cdot, f, x_{0}\right)
$$

uniformly in any $\left[T_{1}, T_{2}\right] \subset I_{f, x_{0}}$;

(ii) in addition, if $\left(F_{n}\right)_{n \in \mathbb{N}}$ in $B$ converges to $F$ in $\left(\mathfrak{W} \Theta \mathfrak{C}\left(\mathbb{R}^{N \times N}\right), \sigma_{\Theta}\right),\left(h_{n}\right)_{n \in \mathbb{N}}$ in $C$ converges to $h$ in $\left(\mathfrak{W} \Theta \mathfrak{C}, \sigma_{\Theta}\right)$, and $\left(y_{0, n}\right)_{n \in \mathbb{N}}$ in $\mathbb{R}^{N}$ converges to $y_{0} \in$ $\mathbb{R}^{N}$, then

$$
y\left(\cdot, f_{n}, F_{n}, h_{n}, x_{0, n}, y_{0, n}\right) \stackrel{n \rightarrow \infty}{\longrightarrow} y\left(\cdot, f, F, h, x_{0}, y_{0}\right)
$$

uniformly in any $\left[T_{1}, T_{2}\right] \subset I_{f, x_{0}}$.

Proof. (i) We will prove the uniform convergence of $\left(x\left(\cdot, f_{n}, x_{0, n}\right)\right)_{n \in \mathbb{N}}$ to $x\left(\cdot, f, x_{0}\right)$ in $[0, T]$ for any $0<T<b_{f, x_{0}}$. The case $a_{f, x_{0}}<T<0$ is analogous. Denote

$$
0<\rho=1+\max \left\{\left(\left|x_{0, n}\right|\right)_{n \in \mathbb{N}},\left\|x\left(\cdot, f, x_{0}\right)\right\|_{L^{\infty}([0, T])}\right\},
$$

and define

$$
z_{n}(t)= \begin{cases}x\left(t, f_{n}, x_{0, n}\right), & \text { if } 0 \leq t<T_{n} \\ x\left(T_{n}, f_{n}, x_{0, n}\right), & \text { if } T_{n} \leq t \leq T\end{cases}
$$

where $T_{n}=\sup \left\{t \in[0, T]|| x\left(s, f_{n}, x_{0, n}\right) \mid \leq \rho, \forall s \in[0, t]\right\}$. Notice that by (3.3) and by the continuity of $\left(x\left(\cdot, f_{n}, x_{0, n}\right)\right)_{n \in \mathbb{N}}$, we have that $T_{n}>0$ for any $n \in \mathbb{N}$. In particular notice that $\left(z_{n}(\cdot)\right)_{n \in \mathbb{N}}$ is uniformly bounded. Moreover, consider $j \in \mathbb{N}$ so that $\rho<j$ and let $\left(m_{n}(\cdot)\right)_{n \in \mathbb{N}}=\left(m_{f_{n}}^{j}(\cdot)\right)_{n \in \mathbb{N}}$ be the sequence of optimal $m$-bounds of $\left(f_{n}\right)_{n \in \mathbb{N}}$ on $B_{j}$. If $t_{1}, t_{2} \in\left[0, T_{n}\right), t_{1}<t_{2}$, then

$$
\left|z_{n}\left(t_{1}\right)-z_{n}\left(t_{2}\right)\right| \leq \int_{t_{1}}^{t_{2}}\left|f_{n}\left(s, z_{n}(s)\right)\right| d s \leq \int_{t_{1}}^{t_{2}} m_{n}(s) d s .
$$

Fixed $\varepsilon>0$, since $E$ admits $L_{l o c}^{1}$-equicontinuous $m$-bounds, there exists $\delta=$ $\delta(T, \varepsilon)>0$ such that, if $0 \leq t_{1} \leq t_{2}<T_{n}$, then the right-hand side in (3.5) is smaller than $\varepsilon$ whenever $t_{2}-t_{1}<\delta$. Notice that, in fact, the inequality $\left|z_{n}\left(t_{1}\right)-z_{n}\left(t_{2}\right)\right|<\varepsilon$ is true on the whole interval $[0, T]$ whenever $t_{2}-t_{1}<\delta$ because in $\left[T_{n}, T\right]$ the difference on the left-hand side of equation (3.5) is zero. Thus, the sequence $\left(z_{n}(\cdot)\right)_{n \in \mathbb{N}}$ is equicontinuous. Then, Ascoli-Arzelá's theorem implies that, up to a subsequence, $\left(z_{n}(\cdot)\right)_{n \in \mathbb{N}}$ converges uniformly to some continuous 
function $z:[0, T] \rightarrow \mathbb{R}^{N}$. In order to conclude the proof, we prove that $z(\cdot) \equiv$ $x\left(\cdot, f, x_{0}\right)$ in $[0, T]$. Define

$$
T_{0}=\sup \{t \in[0, T]|| z(s) \mid<\rho-1 / 2 \quad \forall s \in[0, t]\},
$$

and notice that $T_{0}>0$ because $\left(x_{0, n}\right)_{n \in \mathbb{N}}$ converges to $x_{0}$ and $z(\cdot)$ is continuous. Since $z_{n}(\cdot)$ converges uniformly to $z(\cdot)$ in $[0, T]$, then there exists $n_{0} \in \mathbb{N}$ such that if $n>n_{0}$, then

$$
\left|z_{n}(t)\right|<\rho-1 / 4 \quad \forall t \in\left[0, T_{0}\right] .
$$

Therefore, for any $t \in\left[0, T_{0}\right]$ and for any $n>n_{0}$ one has $z_{n}(t)=x\left(t, f_{n}, x_{0, n}\right)$ and thus

$$
z_{n}(t)=x_{0, n}+\int_{0}^{t} f_{n}\left(s, z_{n}(s)\right) d s, \quad t \in\left[0, T_{0}\right], n>n_{0} .
$$

Since we already know that $z_{n}(\cdot)$ converges uniformly to $z(\cdot)$ in $\left[0, T_{0}\right]$, if we prove that for any $t \in\left[0, T_{0}\right]$ one has

$$
\lim _{n \rightarrow \infty} \int_{0}^{t} f_{n}\left(s, z_{n}(s)\right) d s=\int_{0}^{t} f(s, z(s)) d s,
$$

then, passing to the limit as $n \rightarrow \infty$ in (3.7), we would have that $z(\cdot)$ is a solution of the limit problem $\dot{x}=f(t, x), x(0)=x_{0}$ in $\left[0, T_{0}\right]$. Let us fix $t \in\left[0, T_{0}\right] \cap \mathbb{Q}$ and consider the compact set $\mathcal{K}=\left\{z_{n}(\cdot) \mid n \in \mathbb{N}\right\} \cup\{z(\cdot)\} \subset C\left([0, t], \mathbb{R}^{N}\right)$. Notice that $\mathcal{K} \subset \mathcal{K}_{j}^{[0, t]}$ for the previously identified $j \in \mathbb{N}$ and thus one has

$$
\begin{aligned}
& \left|\int_{0}^{t}\left[f_{n}\left(s, z_{n}(s)\right)-f(s, z(s))\right] d s\right| \\
& \quad \leq\left|\int_{0}^{t}\left[f_{n}\left(s, z_{n}(s)\right)-f\left(s, z_{n}(s)\right)\right] d s\right|+\left|\int_{0}^{t}\left[f\left(s, z_{n}(s)\right)-f(s, z(s))\right] d s\right| \\
& \quad \leq \sup _{y(\cdot) \in \mathcal{K}_{j}^{[0, t]}}\left|\int_{0}^{t}\left[f_{n}(s, y(s))-f(s, y(s))\right] d s\right|+\int_{0}^{t} l_{f}^{j}(s)\left|z_{n}(s)-z(s)\right| d s,
\end{aligned}
$$

where $l_{f}^{j}(\cdot) \in L_{l o c}^{1}$ is the optimal $l$-bound for $f$ on $B_{j}$. The right-hand side of the previous chain of inequalities goes to zero as $n \rightarrow \infty$ because $\left(f_{n}\right)_{n \in \mathbb{N}}$ converges to $f$ in $\sigma_{\Theta}$, and $\left(z_{n}(\cdot)\right)_{n \in \mathbb{N}}$ converges uniformly to $z(\cdot)$ in $[0, T]$. Hence, from (3.7), (3.8) and recalling that $\left(x_{0, n}\right)_{n \in \mathbb{N}}$ converges to $x_{0}$ as $n \rightarrow \infty$, one has

$$
z(t)=x_{0}+\int_{0}^{t} f(s, z(s)) d s \quad \text { for } t \in\left[0, T_{0}\right] \cap \mathbb{Q} .
$$

As a matter of fact, the equality holds on the whole interval $\left[0, T_{0}\right]$. Indeed, for any $t \in\left[0, T_{0}\right]$, if $\left(q_{n}\right)_{n \in \mathbb{N}}$ is a sequence in $\mathbb{Q}$ such that $q_{n} \uparrow t$ as $n \rightarrow \infty$, then, using (3.9) one has

$$
\begin{aligned}
\left|z(t)-z_{0}-\int_{0}^{t} f(s, z(s)) d s\right| & \leq\left|z(t)-z\left(q_{n}\right)\right|+\left|\int_{q_{n}}^{t} f(s, z(s)) d s\right| \\
& \leq\left|z(t)-z\left(q_{n}\right)\right|+\int_{q_{n}}^{t} m_{f}^{j}(s) d s
\end{aligned}
$$


where $m_{f}^{j}(\cdot) \in L_{l o c}^{1}$ is the optimal $m$-bound for $f$ on $B_{j}$. Thus, one obtains (3.9) for all $t \in\left[0, T_{0}\right]$ due to the continuity of $z(\cdot)$ and of the Lebesgue integral. Therefore, $z(\cdot)$ coincides with $x\left(\cdot, f, x_{0}\right)$ on $\left[0, T_{0}\right]$. The only thing left to end the proof is to prove that $T_{0}=T$. Assume, on the contrary, that $T_{0}<T$. Then, by (3.6) and by the continuity of $z(\cdot)$, one would have

$$
\left|z\left(T_{0}\right)\right|=\left|x\left(T_{0}, f, x_{0}\right)\right|=\rho-1 / 2,
$$

which contradicts (3.3). Hence, $T_{0}=T$, as claimed, and thus for any $t \in[0, T]$ we have that $x\left(t, f, x_{0}\right)=z(t)$ and $x\left(t, f_{n}, x_{0, n}\right)=z_{n}(t)$ for any $n \in \mathbb{N}$, which concludes the proof of (i).

(ii) In order to simplify the notation, let us denote by $x_{n}(\cdot)=x\left(\cdot, f_{n}, x_{0, n}\right)$, $y_{n}(\cdot)=y\left(\cdot, f_{n}, F_{n}, h_{n}, x_{0, n}, y_{0, n}\right), x(\cdot)=x\left(\cdot, f, x_{0}\right)$, and $y(\cdot)=y\left(\cdot, f, F, h, x_{0}, y_{0}\right)$. From (i) we have the continuous dependence in the first component and also that there exists $\rho>0$ such that for every $n \in \mathbb{N}$ the following inequality holds: $\left\|x_{n}(\cdot)\right\|_{L^{\infty}([0, T])}<\rho<\infty$. Consider $j \in \mathbb{N}$ such that $j>\rho$, and for any $n \in \mathbb{N}$ let $m_{F_{n}}^{j}$ and $m_{h_{n}}^{j}$ respectively denote the $m$-bounds on $B_{j}$ for $F_{n}$ and $h_{n}$ satisfying the assumptions of $L_{l o c}^{1}$-equicontinuity. We will prove that, for every $0<T<b_{f, x_{0}}$, one has

$$
\left\|y_{n}(\cdot)-y(\cdot)\right\|_{L^{\infty}([0, T])} \stackrel{n \rightarrow \infty}{\longrightarrow} 0 .
$$

For every $n \in \mathbb{N}$ define the function $g_{n}: \mathbb{R} \times \mathbb{R}^{N} \rightarrow \mathbb{R}^{N}$ by

$$
g_{n}(t, y)= \begin{cases}F_{n}\left(t, x_{n}(t)\right) y+h_{n}\left(t, x_{n}(t)\right), & \text { if } 0 \leq t \leq T \\ 0 & \text { otherwise }\end{cases}
$$

and similarly define $g: \mathbb{R} \times \mathbb{R}^{N} \rightarrow \mathbb{R}^{N}$ by

$$
g(t, y)= \begin{cases}F(t, x(t)) y+h(t, x(t)), & \text { if } 0 \leq t \leq T \\ 0 & \text { otherwise }\end{cases}
$$

It is easy to check that such functions are in $\mathfrak{L} \mathfrak{C}$. Furthermore, notice that the solutions of the Cauchy problems

$$
\left\{\begin{array} { l } 
{ \dot { y } = g ( t , y ) } \\
{ y ( 0 ) = y _ { 0 } }
\end{array} \quad \text { and } \quad \left\{\begin{array}{l}
\dot{y}=g_{n}(t, y) \\
y(0)=y_{0, n}
\end{array} \text { for } n \in \mathbb{N},\right.\right.
$$

coincide on $[0, T]$ with $y(\cdot)$ and $y_{n}(\cdot)$, for $n \in \mathbb{N}$, respectively.

For almost every $t \in \mathbb{R}$ and every $y \in \mathbb{R}^{N}$, and up to rescaling $m_{F_{n}}^{j}(\cdot)$ using the equivalence of the norms on the space of matrices $\mathbb{R}^{N \times N}$, one has that

$$
\left|g_{n}(t, y)\right| \leq m_{F_{n}}^{j}(t)|y|+m_{h_{n}}^{j}(t) \quad \text { and } \quad|g(t, y)| \leq m_{F}^{j}(t)|y|+m_{h}^{j}(t) .
$$

Then, from the assumptions on $B \subset \mathfrak{W} \Theta \mathfrak{C}\left(\mathbb{R}^{N \times N}\right)$ and $C \subset \mathfrak{W} \Theta \mathfrak{C}$ and from Proposition 2.29, we have that the set $\left\{g_{n} \mid n \in \mathbb{N}\right\} \cup\{g\}$ has $L_{l o c}^{1}$-equicontinuous 
$m$-bounds and thus a specific suitable set of moduli of continuity $\widetilde{\Theta}$ can be defined as in Definition 3.2. Therefore, if we were able to prove that $g_{n} \stackrel{n \rightarrow \infty}{\longrightarrow} g$ in $\left(\mathfrak{L} \mathfrak{C}, \sigma_{\widetilde{\Theta}}\right)$, we would have that the assumptions of part (i) would be satisfied by the systems in (3.10) and thus, by the fact that the solutions of such systems respectively coincide with $y(\cdot)$ and $y_{n}(\cdot)$, for $n \in \mathbb{N}$, on $[0, T]$, we would obtain the thesis. However, fixed any dense and numerable subset $D$ of $\mathbb{R}^{N}$, we can use the equivalence of the topologies and employ the topology $\sigma_{D}$ instead of $\sigma_{\widetilde{\Theta}}$. Indeed, for almost every $t \in \mathbb{R}$ and every $y_{1}, y_{2} \in \mathbb{R}^{N}$, one has that

$\left|g_{n}\left(t, y_{1}\right)-g_{n}\left(t, y_{2}\right)\right| \leq m_{F_{n}}^{j}(t)\left|y_{1}-y_{2}\right|$ and $\left|g\left(t, y_{1}\right)-g\left(t, y_{2}\right)\right| \leq m_{F}^{j}(t)\left|y_{1}-y_{2}\right|$.

Hence, once again from the assumptions on $B \subset \mathfrak{W} \Theta \mathfrak{C}\left(\mathbb{R}^{N \times N}\right)$ and from Proposition 2.29, we have that the set $\left\{g_{n} \mid n \in \mathbb{N}\right\} \cup\{g\}$ has $L_{l o c}^{1}$-equicontinuous $l$-bounds and thus, by Remark 2.18 and Theorem 2.33, the topology $\sigma_{\widetilde{\Theta}}$ coincide with $\sigma_{D}$ on the set $\left\{g_{n} \mid n \in \mathbb{N}\right\} \cup\{g\}$. Therefore, in order to finish the proof we only need to check that $g_{n} \stackrel{n \rightarrow \infty}{\longrightarrow} g$ in $\left(\mathfrak{L C}, \sigma_{D}\right)$, i.e. that for any $I=\left[q_{1}, q_{2}\right]$, with $q_{1}, q_{2} \in \mathbb{Q}$, and $y \in D$ one has

$$
\lim _{n \rightarrow \infty}\left|\int_{I}\left[g_{n}(t, y)-g(t, y)\right] d t\right|=0
$$

Denote by $I_{T}$ the interval $I \cap[0, T]$. Recalling that the functions $\left(g_{n}\right)_{n \in \mathbb{N}}$ and $g$ are zero outside the interval $[0, T]$, if $I_{T}=\varnothing$, then (3.12) trivially holds true. If $T \notin I$ or if $T \in I \cap \mathbb{Q}$, then the interval $I_{T}$ has endpoints in $\mathbb{Q}$ and one has

$$
\begin{aligned}
& \left|\int_{I}\left[g_{n}(t, y)-g(t, y)\right] d t\right| \\
& \leq\left|\int_{I_{T}}\left[F_{n}\left(t, x_{n}(t)\right)-F(t, x(t))\right] y d t\right|+\left|\int_{I_{T}}\left[h_{n}\left(t, x_{n}(t)\right)-h(t, x(t))\right] d t\right| \\
& \leq|y|\left|\int_{I_{T}}\left[F_{n}\left(t, x_{n}(t)\right)-F\left(t, x_{n}(t)\right)\right] d t\right|+|y|\left|\int_{I_{T}}\left[F\left(t, x_{n}(t)\right)-F(t, x(t))\right] d t\right| \\
& \quad+\left|\int_{I_{T}}\left[h_{n}\left(t, x_{n}(t)\right)-h\left(t, x_{n}(t)\right)\right] d t\right|+\left|\int_{I_{T}}\left[h\left(t, x_{n}(t)\right)-h(t, x(t))\right] d t\right| \\
& \leq \sup _{z(\cdot) \in \mathcal{K}_{j}^{I_{T}}}\left[|y|\left|\int_{I_{T}}\left[F_{n}(t, z(t))-F(t, z(t))\right] d t\right|+\left|\int_{I_{T}}\left[h_{n}(t, z(t))-h(t, z(t))\right] d t\right|\right] \\
& \quad+|y|\left|\int_{I_{T}}\left[F\left(t, x_{n}(t)\right)-F(t, x(t))\right] d t\right|+\left|\int_{I_{T}}\left[h\left(t, x_{n}(t)\right)-h(t, x(t))\right] d t\right| .
\end{aligned}
$$

In the last step of the previous chain of inequalities, the first two integrals go to zero as $n \rightarrow \infty$ because, by assumption, $F_{n} \stackrel{n \rightarrow \infty}{\longrightarrow} F$ in $\left(\mathfrak{W} \Theta \mathfrak{C}\left(\mathbb{R}^{N \times N}\right), \sigma_{\Theta}\right)$ and $h_{n} \stackrel{n \rightarrow \infty}{\longrightarrow} h$ in $\left(\mathfrak{W} \Theta \mathfrak{C}, \sigma_{\Theta}\right)$, whereas the last two integrals go to zero as $n \rightarrow \infty$, because $F \in \mathfrak{W} \Theta \mathfrak{C}\left(\mathbb{R}^{N \times N}\right), h \in \mathfrak{W} \Theta \mathfrak{C}$, and $\left(x_{n}(\cdot)\right)_{n \in \mathbb{N}}$ converges uniformly to $x(\cdot)$ on $[0, T]$ (see Definition 2.4).

The only remaining case is for $T \in I$ but $T \notin \mathbb{Q}$. Then, fixed $\varepsilon>0$ and $k \in \mathbb{N}$ 
consider $q \in I \cap \mathbb{Q}$ such that

$$
\sup _{G \in\left\{g_{n} \mid n \in \mathbb{N}\right\} \cup\{g\}} \int_{q}^{T} m_{G}^{k}(t) d t<\varepsilon,
$$

where, for any $G \in\left\{g_{n} \mid n \in \mathbb{N}\right\} \cup\{g\}, m_{G}^{k}(\cdot) \in L_{l o c}^{1}$ is the optimal $m$-bound for $G$ on $B_{k}$, and the previous inequality holds true since $\left\{g_{n} \mid n \in \mathbb{N}\right\} \cup\{g\}$ has $L_{l o c}^{1}$-equicontinuous $m$-bounds (due to (3.11) and Proposition 2.29). Then, considering $k \in \mathbb{N}$ such that $|y|<k$, we obtain

$$
\begin{aligned}
\left|\int_{I}\left[g_{n}(t, y)-g(t, y)\right] d t\right| \leq 2 \varepsilon & +\sup _{z(\cdot) \in \mathcal{K}_{j}^{I \cap[0, q]}}\left|\int_{I \cap[0, q]}\left[F_{n}(t, z(t))-F(t, z(t))\right] d t\right| \\
& +\sup _{z(\cdot) \in \mathcal{K}_{j}^{I \cap[0, q]}}\left|\int_{I \cap[0, q]}\left[h_{n}(t, z(t))-h(t, z(t))\right] d t\right| \\
& +|y|\left|\int_{I \cap[0, q]}\left[F\left(t, x_{n}(t)\right)-F(t, x(t))\right] d t\right| \\
& +\left|\int_{I \cap[0, q]}\left[h\left(t, x_{n}(t)\right)-h(t, x(t))\right] d t\right| .
\end{aligned}
$$

And again, from the fact that $F_{n} \stackrel{n \rightarrow \infty}{\longrightarrow} F$ in $\left(\mathfrak{W} \Theta \mathfrak{C}\left(\mathbb{R}^{N \times N}\right), \sigma_{\Theta}\right)$ and $h_{n} \stackrel{n \rightarrow \infty}{\longrightarrow} h$ in $\left(\mathfrak{W} \Theta \mathfrak{C}, \sigma_{\Theta}\right)$, and since $F \in \mathfrak{W} \Theta \mathfrak{C}\left(\mathbb{R}^{N \times N}\right), h \in \mathfrak{W} \Theta \mathfrak{C}$, and $\left(x_{n}(\cdot)\right)_{n \in \mathbb{N}}$ converges uniformly to $x(\cdot)$ on $[0, T]$, and from the arbitrariness of $\varepsilon$, we have that the righthand side of the previous inequality goes to zero as $n \rightarrow \infty$. Hence, $g_{n} \stackrel{n \rightarrow \infty}{\longrightarrow} g$ in $\left(\mathfrak{L} \mathfrak{C}, \sigma_{D}\right)$ and thus also in $\left(\mathfrak{L} \mathfrak{C}, \sigma_{\widetilde{\Theta}}\right)$. As a consequence, the assumptions of part (i) are now satisfied by the systems in (3.10) whose solutions respectively coincide on $[0, T]$ with $y(\cdot)$ and $y_{n}(\cdot)$, for $n \in \mathbb{N}$, and thus we obtain the thesis.

Consider $f \in \mathfrak{L} \mathfrak{C}$, a suitable set of moduli of continuity $\Theta=\left(\theta_{j}\right)_{j \in \mathbb{N}}$, and the family of differential equations $\dot{x}=g(t, x)$, where $g \in \operatorname{Hull}_{\left(\mathfrak{L C}, \sigma_{\Theta}\right)}(f)$. With the notation introduced in Theorem 1.2 , let us denote by $\mathcal{U}_{1}$ the subset of $\mathbb{R} \times$ $\operatorname{Hull}_{\left(\mathfrak{L C}, \sigma_{\Theta}\right)}(f) \times \mathbb{R}^{N}$ given by

$$
\mathcal{U}_{1}=\bigcup_{\substack{g \in \operatorname{Hull}_{\left(\mathfrak{L C}, \sigma_{\Theta}\right)}(f), x \in \mathbb{R}^{N}}}\left\{(t, g, x) \mid t \in I_{g, x}\right\} .
$$

Analogously, let $f \in \mathfrak{L} \mathfrak{C}, F \in \mathfrak{W} \Theta \mathfrak{C}\left(\mathbb{R}^{N \times N}\right)$ and $h \in \mathfrak{W} \Theta \mathfrak{C}$, where $\Theta=\left(\theta_{j}\right)_{j \in \mathbb{N}}$ is a suitable set of moduli of continuity, and consider the family of differential equations of the type (3.2) for

$$
(g, G, k) \in \mathbb{H}=\operatorname{Hull}_{\left(\mathfrak{L C} \times \mathfrak{W} \Theta \mathfrak{C} \times \mathfrak{W} \Theta \mathfrak{C}, \sigma_{\Theta} \times \sigma_{\Theta} \times \sigma_{\Theta}\right)}(f, F, h),
$$

where the hull is constructed as in Definition 1.10. Then, we denote by $\mathcal{U}_{2}$ the subset of $\mathbb{R} \times \mathbb{H} \times \mathbb{R}^{N} \times \mathbb{R}^{N}$ given by

$$
\mathcal{U}_{2}=\bigcup_{\substack{(g, G, k) \in \mathbb{H} \\ x_{0} \in \mathbb{R}^{N}}}\left\{\left(t, g, G, k, x_{0}, y_{0}\right) \mid t \in I_{g, x_{0}}, y_{0} \in \mathbb{R}^{n}\right\}
$$


With the previous notation we can state the following theorem.

Theorem 3.5. Let the functions $f \in \mathfrak{L} \mathfrak{C}, F \in \mathfrak{W} \Theta \mathfrak{C}\left(\mathbb{R}^{N \times N}\right)$ and $h \in \mathfrak{W} \Theta \mathfrak{C}$ have

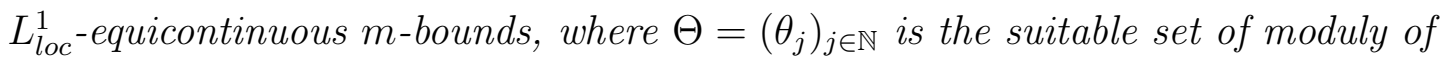
continuity given by the m-bounds of $f$ as shown in Remark 3.3.

(i) The set $\mathcal{U}_{1}$ is open in $\mathbb{R} \times \operatorname{Hull}_{\left(\mathfrak{L C}, \sigma_{\Theta}\right)}(f) \times \mathbb{R}^{N}$ and the map

$$
\begin{array}{rlc}
\Pi: \mathcal{U}_{1} \subset \mathbb{R} \times \operatorname{Hull}_{\left(\mathfrak{L C}, \sigma_{\Theta}\right)}(f) \times \mathbb{R}^{N} & \rightarrow \operatorname{Hull}_{\left(\mathfrak{L C}, \sigma_{\Theta}\right)}(f) \times \mathbb{R}^{N} \\
\left(t, g, x_{0}\right) & \mapsto & \left(g_{t}, x\left(t, g, x_{0}\right)\right)
\end{array}
$$

defines a local continuous skew-product flow on $\operatorname{Hull}_{\left(\mathfrak{L C}, \sigma_{\Theta}\right)}(f) \times \mathbb{R}^{N}$.

(ii) The set $\mathcal{U}_{2}$ is open in $\mathbb{R} \times \mathbb{H} \times \mathbb{R}^{N} \times \mathbb{R}^{N}$ and the map

$$
\begin{aligned}
\Psi: \mathcal{U}_{2} \subset \mathbb{R} \times \mathbb{H} \times \mathbb{R}^{N} \times \mathbb{R}^{N} & \rightarrow \quad \mathbb{H} \times \mathbb{R}^{N} \times \mathbb{R}^{N} \\
\left(t, g, G, k, x_{0}, y_{0}\right) & \mapsto\left(g_{t}, G_{t}, k_{t}, x\left(t, g, x_{0}\right), y\left(t, g, G, k, x_{0}, y_{0}\right)\right)
\end{aligned}
$$

defines a local continuous skew-product flow on $\mathbb{H} \times \mathbb{R}^{N} \times \mathbb{R}^{N}$.

Proof. (i) Firstly, notice that the property of group and of identity at time zero are trivially satisfied, and the continuity in the first component of the image is given by Corollary 2.45.

As regards the second component, it is enough to show that the assumptions of Theorem 3.4 apply to $E=\operatorname{Hull}_{\left(\mathfrak{L C}, \mathcal{T}_{\ominus}\right)}(f)$. To the aim, we check that the set of moduli of continuity given by the $m$-bounds of $f$ as in Remark 3.3, also turns out to be the set of moduli of continuity given by the $m$-bounds of the functions in $\operatorname{Hull}_{\left(\mathfrak{L C}, \mathcal{T}_{\Theta}\right)}(f)$ as in Definition 3.2. Thus, fix $j \in \mathbb{N}$ and consider $g \in \operatorname{Hull}_{\left(\mathfrak{L C}, \sigma_{\Theta}\right)}(f)$ and a sequence $\left(\tau_{n}\right)_{n \in \mathbb{N}}$ in $\mathbb{R}$ such that $f_{\tau_{n}} \stackrel{n \rightarrow \infty}{\longrightarrow} g$. Moreover, denote by $m^{j}(\cdot)=m_{f}^{j}(\cdot)$ the optimal $m$-bound for $f$ on $B_{j}$; as shown in (2.14), one has that for every $n \in \mathbb{N}$, the optimal $m$-bound of the $\tau_{n}$-time translation of $f$ is the $\tau_{n}$-time translation of the optimal $m$-bound of $f$, that is

$$
\forall n \in \mathbb{N}: \quad m_{f_{\tau_{n}}}^{j}(\cdot)=m_{\tau_{n}}^{j}(\cdot) .
$$

Reasoning as in the first part of the proof of Proposition 2.26 we have that the sequence of absolutely continuous measures with densities $\left(m_{\tau_{n}}^{j}(\cdot)\right)_{n \in \mathbb{N}}$ vaguely converges, up to a subsequence, to a positive measure whose absolutely continuous part has density $m_{g}^{j}(\cdot) \in L_{l o c}^{1}$, and $m_{g}^{j}(\cdot)$ is an $m$-bound for $g$ on $B_{j}$. Furthermore, for any $t \in \mathbb{R}, h>0$ and $\delta>0$, one can consider a function $\phi \in C_{C}^{+}$such that $\operatorname{supp} \phi \subset[t-\delta, t+h+\delta]$ and $\phi \equiv 1$ in $[t, t+h]$ and write

$$
\int_{t}^{t+h} m_{g}^{j}(u) d u \leq \int_{\mathbb{R}} \phi(u) m_{g}^{j}(u) d u=\lim _{n \rightarrow \infty} \int_{\mathbb{R}} \phi(u) m_{\tau_{n}}^{j}(u) d u \leq \sup _{\tau \in \mathbb{R}} \int_{t-\delta}^{t+h+\delta} m_{\tau}^{j}(u) d u,
$$

and thus, by the arbitrariness on $\delta$ we get

$$
\int_{t}^{t+h} m_{g}^{j}(u) d u \leq \sup _{\tau \in \mathbb{R}} \int_{t}^{t+h} m_{\tau}^{j}(u) d u \leq \theta_{j}(h) .
$$


Then, the sets of moduli of continuity coincide, as stated, and applying Theorem 3.4 we obtain the result.

(ii) Analogous reasonings apply to this case.

The following theorem addresses the same problem, i.e. the continuity of the skew product semiflow, but the strong topology $\mathcal{T}_{\Theta}$ is employed. Particularly, in the following theorem, the triangular problem is considered in a smaller space with a stronger topology (with respect to Theorem 3.4) but no assumptions on the $m$-bounds of the functions defining the vector field of the linear problem, are necessary anymore.

Theorem 3.6. Consider $E \subset \mathfrak{L C}_{p}$ with $L_{\text {loc }}^{1}$-equicontinuous $m$-bounds and let $\Theta$ be the countable set of moduli of continuity in Definition 3.2. With the notation of Theorem 1.2 and Corollary 3.1,

(i) if $\left(f_{n}\right)_{n \in \mathbb{N}}$ in $E$ converges to $f$ in $\left(\mathfrak{L} \mathfrak{C}_{p}, \mathcal{T}_{\Theta}\right)$ and $\left(x_{0, n}\right)_{n \in \mathbb{N}}$ in $\mathbb{R}^{N}$ converges to $x_{0} \in \mathbb{R}^{N}$, then

$$
x\left(\cdot, f_{n}, x_{0, n}\right) \stackrel{n \rightarrow \infty}{\longrightarrow} x\left(\cdot, f, x_{0}\right)
$$

uniformly in any $\left[T_{1}, T_{2}\right] \subset I_{f, x_{0}}$;

(ii) moreover, if $\left(F_{n}\right)_{n \in \mathbb{N}}$ in $\Theta \mathfrak{C}_{p}\left(\mathbb{R}^{N \times N}\right)$ converges to $F$ in $\left(\Theta \mathfrak{C}_{p}\left(\mathbb{R}^{N \times N}\right), \mathcal{T}_{\Theta}\right)$, $\left(h_{n}\right)_{n \in \mathbb{N}}$ in $\Theta \mathfrak{C}_{p}$ converges to $h$ in $\left(\Theta \mathfrak{C}_{p}, \mathcal{T}_{\Theta}\right)$, and $\left(y_{0, n}\right)_{n \in \mathbb{N}}$ in $\mathbb{R}^{N}$ converges to $y_{0} \in \mathbb{R}^{N}$, then

$$
y\left(\cdot, f_{n}, F_{n}, h_{n}, x_{0, n}, y_{0, n}\right) \stackrel{n \rightarrow \infty}{\longrightarrow} y\left(\cdot, f, F, h, x_{0}, y_{0}\right)
$$

uniformly in any $\left[T_{1}, T_{2}\right] \subset I_{f, x_{0}}$.

Proof. (i) Notice that for any $1 \leq p<\infty$ if $f_{n} \stackrel{n \rightarrow \infty}{\longrightarrow} f$ in $\left(\mathfrak{L C}_{p}, \mathcal{T}_{\Theta}\right)$, then it also converges in $\left(\mathfrak{L} \mathfrak{C}, \sigma_{\Theta}\right)$. Therefore, a careful look at the proof of Theorem 3.4 shows that (i) of such theorem applies.

(ii) The continuous dependence in the first component is given by part (i). Let us simplify the notation, as follows

$$
\begin{aligned}
& x_{n}(\cdot)=x\left(\cdot, f_{n}, x_{0, n}\right), \quad y_{n}(\cdot)=y\left(\cdot, f_{n}, F_{n}, h_{n}, x_{0, n}, y_{0, n}\right), \\
& x(\cdot)=x\left(\cdot, f, x_{0}\right), \quad \text { and } \quad y(\cdot)=y\left(\cdot, f, F, h, x_{0}, y_{0}\right) .
\end{aligned}
$$

From part (i) we already have that $\left(x_{n}(\cdot)\right)_{n \in \mathbb{N}}$ converges uniformly to $x(\cdot)$ on the compact subsets of $I_{f, x_{0}}$. Moreover, call $\widetilde{F}_{n}(t)=F_{n}\left(t, x_{n}(t)\right), \widetilde{F}(t)=F(t, x(t))$, $\widetilde{h}_{n}(t)=h_{n}\left(t, x_{n}(t)\right)$ and $\widetilde{h}(t)=h(t, x(t))$. If we prove that $\left(\widetilde{F}_{n}(\cdot)\right)_{n \in \mathbb{N}}$ and $\left.\widetilde{h}_{n}(\cdot)\right)_{n \in \mathbb{N}}$ converge in $L_{l o c}^{p}$ to $\widetilde{F}(\cdot)$ and $\widetilde{h}(\cdot)$ respectively, then we have the thesis 
applying Lemma IV.9 in [53] to the linear case. Therefore, let us fix an interval $I \subset \mathbb{R}$. Then,

$$
\begin{aligned}
\left\|\widetilde{F}_{n}(\cdot)-\widetilde{F}(\cdot)\right\|_{p} & =\left\|F_{n}\left(\cdot, x_{n}(\cdot)\right)-F(\cdot, x(\cdot))\right\|_{p} \\
& \leq\left\|F_{n}\left(\cdot, x_{n}(\cdot)\right)-F\left(\cdot, x_{n}(\cdot)\right)\right\|_{p}+\left\|F\left(\cdot, x_{n}(\cdot)\right)-F(\cdot, x(\cdot))\right\|_{p} \\
& \leq \sup _{\xi \in \mathcal{K}_{j}^{I}}\left\|F_{n}(\cdot, \xi(\cdot))-F(\cdot, \xi(\cdot))\right\|_{p}+\left\|F\left(\cdot, x_{n}(\cdot)\right)-F(\cdot, x(\cdot))\right\|_{p}
\end{aligned}
$$

where $j \in \mathbb{N}$ is chosen so that $j>\max \left\{\|x(\cdot)\|_{L^{\infty}(I)},\left(\left\|x_{n}(\cdot)\right\|_{L^{\infty}(I)}\right)_{n \in \mathbb{N}}\right\}$. The right-hand side of the previous inequality goes to zero as $n \rightarrow \infty$ because $\left(F_{n}\right)_{n \in \mathbb{N}}$ converges to $F$ in $\left(\Theta \mathfrak{C}_{p}\left(\mathbb{R}^{N \times N}\right), \mathcal{T}_{\Theta}\right)$, and $F$ satisfies $(\mathrm{T})$. Analogous reasonings apply to the sequence $\left(\widetilde{h}_{n}(\cdot)\right)_{n \in \mathbb{N}}$. Therefore, we have the required $L_{l o c}^{p}$ convergences and thus uniform convergence of the solutions of the nonhomogeneus linear equation.

Let $f \in \mathfrak{L C}_{p}, \Theta=\left(\theta_{j}\right)_{j \in \mathbb{N}}$ be a suitable set of moduli of continuity and consider the family of differential equations $\dot{x}=g(t, x)$, where $g \in \operatorname{Hull}_{\left(\mathfrak{L C}_{p}, \mathcal{T}_{\Theta}\right)}(f)$. With the notation introduced in Theorem 1.2 , let us denote by $\mathcal{U}_{1}$ the subset of $\mathbb{R} \times \operatorname{Hull}_{\left(\mathfrak{L C} \mathfrak{C}_{p}, \mathcal{T}_{\Theta}\right)}(f) \times \mathbb{R}^{N}$ given by

$$
\mathcal{U}_{1}=\bigcup_{\substack{\left.g \in \operatorname{Hull}_{\left(\mathfrak{L} \mathfrak{c}_{p}, \mathcal{T}_{\Theta}\right)}(f), \mathbb{R}^{N} \\ x \in I_{g, x}\right\}}}\{(t, g, x) \mid t \in
$$

Let $f \in \mathfrak{L C}_{p}, F \in \Theta \mathfrak{C}\left(\mathbb{R}^{N \times N}\right), h \in \Theta \mathfrak{C}$ and consider the family of differential equations of type (3.2) for

$$
(g, G, k) \in \mathbb{H}=\operatorname{Hull}_{\left(\mathfrak{L} \mathfrak{C}_{p} \times \Theta \mathfrak{C} \times \Theta \mathfrak{C}, \mathcal{T}_{\Theta} \times \mathcal{T}_{\Theta} \times \mathcal{T}_{\Theta}\right)}(f, F, h),
$$

where the hull is constructed as in Definition 1.10. Denote by $\mathcal{U}_{2}$ the subset of $\mathbb{R} \times \mathbb{H} \times \mathbb{R}^{N} \times \mathbb{R}^{N}$ given by

$$
\mathcal{U}_{2}=\bigcup_{\substack{(g, G, k) \in \mathbb{H}, x_{0} \in \mathbb{R}^{N}}}\left\{\left(t, g, G, k, x_{0}, y_{0}\right) \mid t \in I_{g, x_{0}}, y_{0} \in \mathbb{R}^{n}\right\}
$$

With the previous notation we can state the following theorem.

Theorem 3.7. Let $f \in \mathfrak{L C}_{p}$ have $L_{\text {loc }}^{1}$-equicontinuous $m$-bounds and let $\Theta=$ $\left(\theta_{j}\right)_{j \in \mathbb{N}}$ be the suitable set of moduli of continuity given by the $m$-bounds as shown in Remark 3.3.

(i) The set $\mathcal{U}_{1}$ is open in $\operatorname{Hull}_{\left(\mathfrak{L C}_{p}, \mathcal{T}_{\Theta}\right)}(f) \times \mathbb{R}^{N}$ and the map

$$
\begin{array}{ccc}
\Phi_{1}: \mathcal{U}_{1} \subset \mathbb{R} \times \operatorname{Hull}_{\left(\mathfrak{L C}_{p}, \mathcal{T}_{\Theta}\right)}(f) \times \mathbb{R}^{N} & \rightarrow \operatorname{Hull}_{\left(\mathfrak{L C} \mathfrak{C}_{p}, \mathcal{T}_{\Theta}\right)}(f) \times \mathbb{R}^{N} \\
\left(t, g, x_{0}\right) & \mapsto \quad\left(g_{t}, x\left(t, g, x_{0}\right)\right),
\end{array}
$$

defines a local continuous skew-product flow on $\operatorname{Hull}_{\left(\mathfrak{L C}_{p}, \mathcal{T}_{\Theta}\right)}(f) \times \mathbb{R}^{N}$. 
(ii) The set $\mathcal{U}_{2}$ is open in $\mathbb{R} \times \mathbb{H} \times \mathbb{R}^{N} \times \mathbb{R}^{N}$ and the map

$$
\begin{aligned}
& \Phi_{2}: \mathcal{U}_{2} \subset \mathbb{R} \times \mathbb{H} \times \mathbb{R}^{N} \times \mathbb{R}^{N} \rightarrow \quad \mathbb{H} \times \mathbb{R}^{N} \times \mathbb{R}^{N} \\
&\left(t, g, G, k, x_{0}, y_{0}\right) \mapsto\left(g_{t}, G_{t}, k_{t}, x\left(t, g, x_{0}\right), y\left(t, g, G, k, x_{0}, y_{0}\right)\right),
\end{aligned}
$$

defines a local continuous skew-product flow on $\mathbb{H} \times \mathbb{R}^{N} \times \mathbb{R}^{N}$.

Proof. The proof is a consequence of Corollary 2.42 and Theorem 3.6, reasoning as in the proof of Theorem 3.5.

Finally, we provide two results of continuity of the solutions for Carathéodory differential systems whose vector fields are in a set $E \subset \mathfrak{L C}_{p}$ with $L_{l o c}^{p}$-bounded l-bounds. Thanks to Theorem 2.31, we have that all the strong topologies introduced in Section 2.2 coincide on $E$. In particular, if $C \subset E$ is compact (and recall that the problem of compactness in $\mathfrak{L} \mathfrak{C}$ has been studied in Section 2.6), then the solutions of the differential equations of type (3.1), whose vector fields belong to $C$, determine a suitable set of moduli of continuity which will be used to obtain the continuity of the solutions of the second equation in the triangular system. Notice that no assumptions on the $m$-bounds are required.

Theorem 3.8. Consider $E \subset \mathfrak{L C}_{p}$ with $L_{\text {loc }}^{p}$-bounded l-bounds.

(i) If $\left(f_{n}\right)_{n \in \mathbb{N}}$ in $E$ converges to $f$ in $\left(\mathfrak{L C}_{p}, \mathcal{T}_{D}\right)$ and $\left(x_{0, n}\right)_{n \in \mathbb{N}}$ in $\mathbb{R}^{N}$ converges to $x_{0} \in \mathbb{R}^{N}$, then

$$
x\left(\cdot, f_{n}, x_{0, n}\right) \stackrel{n \rightarrow \infty}{\longrightarrow} x\left(\cdot, f, x_{0}\right)
$$

uniformly in any $\left[T_{1}, T_{2}\right] \subset I_{f, x_{0}}$.

(ii) Let $C \subset E$ be compact with respect to $\mathcal{T}_{D}$ and, for any interval $I=\left[q_{1}, q_{2}\right] \subset$ $\mathbb{R}$, with $q_{1}, q_{2} \in \mathbb{Q}$, and any $j \in \mathbb{N}$, define

$$
C_{j}^{I}=\left\{\begin{array}{l|c}
x: J \rightarrow B_{j} & \begin{array}{c}
J \subset I \text { interval, and } \\
\exists f \in C \text { such that } \forall s, t \in J \\
x(t)=x(s)+\int_{s}^{t} f(u, x(u)) d u
\end{array}
\end{array}\right\} .
$$

Then, each of the sets $C_{j}^{I}$ is equicontinuous and, denoted by $\theta_{j}^{I}$ its modulus of continuity, the set

$$
\Theta=\left\{\theta_{j}^{I} \in C\left(\mathbb{R}^{+}, \mathbb{R}^{+}\right) \mid \begin{array}{c}
I=\left[q_{1}, q_{2}\right] \subset \mathbb{R}, \text { with } q_{1}, q_{2} \in \mathbb{Q}, j \in \mathbb{N}, \\
\theta_{j}^{I} \text { modulus of continuity of } C_{j}^{I}
\end{array}\right\},
$$

is a suitable set of moduli of continuity. 
(iii) Let $C \subset E$ be compact with respect to $\mathcal{T}_{D}$ and $\Theta$ be the suitable set of moduli of continuity given by (ii). If $\left(f_{n}\right)_{n \in \mathbb{N}}$ in $C$ converges to $f$ in $\left(\mathfrak{L C}_{p}, \mathcal{T}_{D}\right)$, $\left(F_{n}\right)_{n \in \mathbb{N}}$ in $\Theta \mathfrak{C}_{p}\left(\mathbb{R}^{N \times N}\right)$ converges to $F$ in $\left(\Theta \mathfrak{C}_{p}\left(\mathbb{R}^{N \times N}\right), \mathcal{T}_{\Theta}\right),\left(h_{n}\right)_{n \in \mathbb{N}}$ in $\Theta \mathfrak{C}_{p}$ converges to $h$ in $\left(\Theta \mathfrak{C}_{p}, \mathcal{T}_{\Theta}\right)$, and $\left(x_{0, n}, y_{0, n}\right)_{n \in \mathbb{N}}$ in $\mathbb{R}^{N} \times \mathbb{R}^{N}$ converges to $\left(x_{0}, y_{0}\right) \in \mathbb{R}^{N} \times \mathbb{R}^{N}$, then

$$
\left(x\left(\cdot, f_{n}, x_{0, n}\right), y\left(\cdot, f_{n}, F_{n}, h_{n}, x_{0, n}, y_{0, n}\right)\right) \rightarrow\left(x\left(\cdot, f, x_{0}\right), y\left(\cdot, f, F, h, x_{0}, y_{0}\right)\right)
$$

as $n \rightarrow \infty$, uniformly in any $\left[T_{1}, T_{2}\right] \subset I_{f, x_{0}}$.

Proof. (i) Since $E$ has $L_{l o c}^{p}$-bounded l-bounds, by Theorem 2.31 the convergence in $\left(\mathfrak{L} \mathfrak{C}_{p}, \mathcal{T}_{D}\right)$ implies the convergence in $\left(\mathfrak{L} \mathfrak{C}_{p}, \mathcal{T}_{B}\right)$. The proof closely follows the one given in Theorem 3.4, whose notation is hereby inherited. In particular, we focus on the interval $0<T<b_{f, x_{0}}$, since analogous reasonings apply for $a_{f, x_{0}}<T<0$.

After setting the constant $\rho$ as in (3.3), and after defining the sequence of functions $\left(z_{n}(\cdot)\right)_{n \in \mathbb{N}}$ in $C\left([0, T], \mathbb{R}^{N}\right)$ as in $(3.4)$, we notice that $\left(z_{n}(\cdot)\right)_{n \in \mathbb{N}}$ is uniformly bounded and for any $n \in \mathbb{N}$ one also has

$$
\begin{aligned}
\left|z_{n}\left(t_{1}\right)-z_{n}\left(t_{2}\right)\right| & \leq \int_{t_{1}}^{t_{2}}\left|f_{n}\left(s, z_{n}(s)\right)\right| d s \\
& \leq \int_{t_{1}}^{t_{2}}\left|f_{n}\left(s, z_{n}(s)\right)-f\left(s, z_{n}(s)\right)\right| d s+\int_{t_{1}}^{t_{2}}\left|f\left(s, z_{n}(s)\right)\right| d s \\
& \leq \int_{t_{1}}^{t_{2}}\left|f_{n}\left(s, z_{n}(s)\right)-f\left(s, z_{n}(s)\right)\right| d s+\int_{t_{1}}^{t_{2}} m_{f}^{j}(s) d s
\end{aligned}
$$

where $m_{f}^{j}(\cdot) \in L_{l o c}^{p}$ is the optimal $m$-bound for $f$ on $B_{j}$. Fixed $\varepsilon>0$, due to the convergence of $\left(f_{n}\right)_{n \in \mathbb{N}}$ to $f$ in $\left(\mathfrak{L} \mathfrak{C}_{p}, \mathcal{T}_{B}\right)$, and recalling that $\left\{z_{k} \mid k \in \mathbb{N}\right\}$ is a bounded set of continuous functions, we have that there exists an $n_{0} \in \mathbb{N}$ such that

$$
n>n_{0} \Rightarrow \sup _{k \in \mathbb{N}} \int_{t_{1}}^{t_{2}}\left|f_{n}\left(s, z_{k}(s)\right)-f\left(s, z_{k}(s)\right)\right| d s<\varepsilon .
$$

On the other side, by the absolute continuity of the integral, there exists $\delta>0$ such that if $0<t_{2}-t_{1}<\delta$, then

$$
\int_{t_{1}}^{t_{2}}\left|f_{n}\left(s, z_{n}(s)\right)-f\left(s, z_{n}(s)\right)\right| d s<\varepsilon \quad \forall n=1, \ldots, n_{0},
$$

and also

$$
\int_{t_{1}}^{t_{2}} m_{f}^{j}(s) d s<\varepsilon
$$

Gathering the inequalities (3.15), (3.16), (3.17) and (3.18), we obtain a common modulus of continuity for all the functions in $\left\{z_{i} \mid i \in \mathbb{N}\right\}$. Therefore, thanks to 
Ascoli-Arzelà's Theorem, we have that, up to a subsequence, $\left(z_{n}(\cdot)\right)_{n \in \mathbb{N}}$ converges uniformly in $[0, T]$ to a continuous function $z(\cdot)$. At his point, it is possible to prove that $z(\cdot)$ coincide with $x\left(\cdot, f, x_{0}\right)$ on $[0, T]$ following the same arguments of Theorem 3.4, with the only exception that the equality in (3.8) will be guaranteed by the convergence in the strong topology $\mathcal{T}_{B}$, once one notice that $\left\{z_{i}(\cdot) \mid i \in\right.$ $\mathbb{N}\} \cup\{z(\cdot)\}$ is a bounded set.

(ii) Let us fix $\varepsilon>0, I=\left[q_{1}, q_{2}\right] \subset \mathbb{R}$, with $q_{1}, q_{2} \in \mathbb{Q}$, and $j \in \mathbb{N}$, and consider the following seminorm defined on $E$

$$
p_{j}(f)=\sup _{z \in C\left(I, B_{j}\right)} \int_{I}|f(t, z(t))| d t, \quad f \in E .
$$

Moreover, for any $\tilde{f} \in C$, denote by $U_{\varepsilon / 2}^{j}(\widetilde{f})$ the following set

$$
U_{\varepsilon / 2}^{j}(\widetilde{f})=\left\{f \in E \quad \mid p_{j}(f-\widetilde{f}) \leq \varepsilon / 2\right\} .
$$

Therefore, by the compactness of $C$, there exist $\nu \in \mathbb{N}$ and $f_{1}, \ldots, f_{\nu} \in C$ such that

$$
C \subset \bigcup_{i=1}^{\nu} U_{\varepsilon / 2}^{j}\left(f_{i}\right)
$$

For any $i=1, \ldots, \nu$, denote by $m_{i}(\cdot)$ the $m$-bound of $f_{i}$ on $B_{j}$ and notice that there exists $\delta>0$ such that, if $s, t \in I$ and $0 \leq t-s<\delta$, then

$$
\int_{s}^{t} m_{i}(u) d u \leq \frac{\varepsilon}{2}, \quad \forall i=1, \ldots, \nu
$$

Now, consider $x: J \rightarrow B_{j}$, with $x(\cdot) \in C_{j}^{I}$ and possibly extend it by constants to the whole interval $I$. Also, by the definition of $C_{j}^{I}$ in $(3.14), x(\cdot)$ determines $f \in C$ such that

$$
x(t)=x(s)+\int_{s}^{t} f(u, x(u)) d u, \quad \text { for every } s, t \in J .
$$

Moreover, up to a reordering of the functions $f_{1}, \ldots, f_{\nu}$ whose $\varepsilon / 2$-neighborhoods provide a covering of $C$, assume that $p_{j}\left(f-f_{1}\right) \leq \varepsilon / 2$. Then, for any $s, t \in J$ with $0 \leq t-s<\delta$ we have

$$
\begin{aligned}
|x(t)-x(s)| & \leq \int_{s}^{t}|f(u, x(u))| d u \\
& =\int_{s}^{t}\left|f(u, x(u))-f_{1}(u, x(u))\right| d u+\int_{s}^{t} m_{1}(u) d u \\
& \leq p_{j}\left(f-f_{1}\right)+\int_{s}^{t} m_{1}(u) d u \leq \varepsilon .
\end{aligned}
$$

Hence, from the arbitrariness of $x(\cdot) \in C_{j}^{I}$, one has that the set $C_{j}^{I}$ is equicontinuous. 
(iii) The proof of this part is the same as the one of part (ii) of Theorem 3.6 with the exception that now $\Theta$ is no more determined by the $m$-bounds of the functions in $E$ but as in the statement. Notice that everything is consistent, since for any $I=\left[q_{1}, q_{2}\right] \subset \mathbb{R}$, with $q_{1}, q_{2} \in \mathbb{Q}$, and for any $j \in \mathbb{N}$, we have that $C_{j}^{I} \subset \mathcal{K}_{j}^{I}$, where the functions in $C_{j}^{I}$ are possibly extended by constants to the whole interval $I$, as before.

Next, we state the result of continuity of the skew-product flow for the topology $\mathcal{T}_{D}$. Notice that, in analogy with Theorem 3.7, we provide a result for both systems like (3.1) and like (3.2) in the respective hulls. However, a major difference in the assumptions of the second case occurs, that is, $\operatorname{Hull}_{\left(\mathfrak{L C}_{p}, \mathcal{T}_{D}\right)}(f)$ is required to be compact, due to the fact that Theorem 3.8(iii) is used to obtain the result. Incidentally, recall that a characterization of compactness of $\operatorname{Hull}_{\left(\mathfrak{L C}_{p}, \mathcal{T}_{D}\right)}(f)$ is given in Corollary 2.36.

As before, let us set some notation first. Considered $f \in \mathfrak{L} \mathfrak{C}_{p}$, let us denote by $\mathcal{U}_{1}$ the subset of $\mathbb{R} \times \operatorname{Hull}_{\left(\mathfrak{L C} \mathfrak{C}_{p}, \mathcal{T}_{D}\right)}(f) \times \mathbb{R}^{N}$ given by

$$
\mathcal{U}_{1}=\bigcup_{\substack{g \in \operatorname{Hull}_{\left(\mathfrak{s} \mathfrak{C}_{p}, \mathcal{T}_{D}\right)}(f) \\ x_{0} \in \mathbb{R}^{N}}}\left\{\left(t, g, x_{0}\right) \mid t \in I_{g, x_{0}}\right\} .
$$

Moreover, considered a suitable set of moduli of continuity $\Theta=\left(\theta_{j}\right)_{j \in \mathbb{N}}$, and the functions $F \in \Theta \mathfrak{C}\left(\mathbb{R}^{N \times N}\right)$ and $h \in \Theta \mathfrak{C}$, let us denote by $\mathbb{H}$ the set given by

$$
\mathbb{H}=\operatorname{Hull}_{\left(\mathfrak{L} \mathfrak{C}_{p} \times \Theta \mathfrak{C} \times \Theta \mathfrak{C}, \mathcal{T}_{D} \times \mathcal{T}_{\Theta} \times \mathcal{T}_{\Theta}\right)}(f, F, h),
$$

and by $\mathcal{U}_{2} \subseteq \mathbb{R} \times \mathbb{H} \times \mathbb{R}^{N} \times \mathbb{R}^{N}$, the set given by

$$
\mathcal{U}_{2}=\bigcup_{\substack{(g, G, k) \in \mathbb{H} \\ x_{0} \in \mathbb{R}^{N}}}\left\{\left(t, g, G, k, x_{0}, y_{0}\right) \mid t \in I_{g, x_{0}}, y_{0} \in \mathbb{R}\right\} .
$$

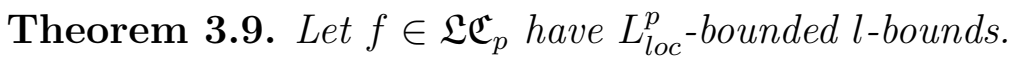

(i) The set $\mathcal{U}_{1}$ is open in $\mathbb{R} \times \operatorname{Hull}_{\left(\mathfrak{L C}_{p}, \mathcal{T}_{D}\right)}(f) \times \mathbb{R}^{N}$ and the map

$$
\begin{aligned}
& \Phi_{1}: \mathcal{U}_{1} \subset \mathbb{R} \times \operatorname{Hull}_{\left(\mathfrak{L C}_{p}, \mathcal{T}_{D}\right)}(f) \times \mathbb{R}^{N} \rightarrow \operatorname{Hull}_{\left(\mathfrak{L C}_{p}, \mathcal{T}_{D}\right)}(f) \times \mathbb{R}^{N}, \\
& \left(t, g, x_{0}\right) \quad \mapsto \quad\left(g_{t}, x\left(t, g, x_{0}\right)\right),
\end{aligned}
$$

defines a local continuous skew-product flow on $\operatorname{Hull}_{\left(\mathfrak{L C}_{p}, \mathcal{T}_{D}\right)}(f) \times \mathbb{R}^{N}$.

(ii) Furthermore, if $\operatorname{Hull}_{\left(\mathfrak{L C}_{p}, \mathcal{T}_{D}\right)}(f)$ is compact, $\Theta$ is the suitable set of moduli of continuity given by Theorem 3.8(ii), $F \in \Theta \mathfrak{C}\left(\mathbb{R}^{N \times N}\right)$, and $h \in \Theta \mathfrak{C}$, then the set $\mathcal{U}_{2}$ is open in $\mathbb{R} \times \mathbb{H} \times \mathbb{R}^{N} \times \mathbb{R}^{N}$, and the map

$$
\begin{aligned}
\Phi_{2}: \mathcal{U}_{2} & \subset \mathbb{R} \times \mathbb{H} \times \mathbb{R}^{N} \times \mathbb{R}^{N} \rightarrow \quad \mathbb{H} \times \mathbb{R}^{N} \times \mathbb{R}^{N} \\
\left(t, g, G, k, x_{0}, y_{0}\right) & \mapsto\left(g_{t}, G_{t}, k_{t}, x\left(t, g, x_{0}\right), y\left(t, g, G, k, x_{0}, y_{0}\right)\right),
\end{aligned}
$$

defines a local continuous skew-product flow on $\mathbb{H} \times \mathbb{R}^{N} \times \mathbb{R}^{N}$. 
Proof. The property of group and of identity at time zero are trivially satisfied. Moreover, the continuity in the base flows in (i) and (ii) are a direct consequence of Corollary 2.42, while the continuity of the solutions is given by Theorem 3.8.

\subsection{Linearized skew-product flows}

In Section 3.1, triangular systems composed of a nonlinear system with vector field in $\mathfrak{L} \mathfrak{C}$ and a linear system with vector field in $\mathfrak{W} \Theta \mathfrak{C}$ or $\Theta \mathfrak{C}$ were treated. This kind of systems assume additional relevance when the linear system is the variational equation of the non-linear one. In particular, (depending on the assumptions on $f$ and on its Jacobian with respect to $x$ ) the previous theorems allow to deduce two different types of skew-product flow and to prove the differentiability of the solutions of the non-linear equations with respect to the initial data also in some cases in which the vector field is not differentiable with respect to $x$, and thus does not admit a classic variational equation. Such results motivate the definition of linearized skew-product flows that we will introduce at the end of the section.

The classic theory of Carathéodory ODEs provides the differentiability of the solutions with respect to the initial conditions when the respective vector fields are continuously differentiable with respect to $x$ (see Kurzweil [37] and Bressan and Piccoli [11]). In order to set some notation and for the sake of completeness we include a proof of such a result.

Theorem 3.10. Let $f \in \mathfrak{L} \mathfrak{C}$ be continuously differentiable with respect to $x$ for a.e. $t \in \mathbb{R}$ and denote by $J_{x} f \in \mathfrak{S C}_{p}\left(\mathbb{R}^{N \times N}\right)$ the Jacobian of $f$ with respect to $x$. If $x\left(\cdot, f, x_{0}\right)$ and $y\left(\cdot, f, J_{x} f, x_{0}, y_{0}\right)$ are respectively the solutions of the Cauchy Problems

$$
\left\{\begin{array} { l } 
{ \dot { x } = f ( t , x ) } \\
{ x ( 0 ) = x _ { 0 } }
\end{array} \quad \text { and } \quad \left\{\begin{array}{l}
\dot{y}=J_{x} f\left(t, x\left(t, f, x_{0}\right)\right) y \\
y(0)=y_{0}
\end{array}\right.\right.
$$

defined on any compact interval $\left[T_{0}, T_{1}\right]$ included in the maximal interval of definition $I_{f, x_{0}}$, then we have that

$$
\lim _{\varepsilon \rightarrow 0^{+}}\left|\frac{x\left(t, f, x_{0}+\varepsilon y_{0}\right)-x\left(t, f, x_{0}\right)}{\varepsilon}-y\left(t, f, J_{x} f, x_{0}, y_{0}\right)\right|=0,
$$

uniformly for $t \in\left[T_{0}, T_{1}\right]$ and $y_{0} \in B_{1}$.

Proof. Let us simplify the notation, denoting by $x\left(\cdot, x_{0}\right)=x\left(\cdot, f, x_{0}\right)$, and by $y\left(\cdot, x_{0}, y_{0}\right)=y\left(\cdot, f, J_{x} f, x_{0}, y_{0}\right)$ and consider the case $t \in\left[0, T_{1}\right] ;$ all the other cases can be worked out analogously. By definition of solution, we have that

$$
\begin{array}{r}
\frac{x\left(t, x_{0}+\varepsilon y_{0}\right)-x\left(t, x_{0}\right)}{\varepsilon}=y_{0}+\frac{1}{\varepsilon} \int_{0}^{t}\left[f\left(s, x\left(s, x_{0}+\varepsilon y_{0}\right)\right)-f\left(s, x\left(s, x_{0}\right)\right)\right] d s \\
=y_{0}+\int_{0}^{t}\left(\int_{0}^{1} J_{x} f\left(s, \xi_{\varepsilon}(s, \alpha)\right) d \alpha\right) \frac{x\left(s, x_{0}+\varepsilon y_{0}\right)-x\left(s, x_{0}\right)}{\varepsilon} d s,
\end{array}
$$


where

$$
\xi_{\varepsilon}(s, \alpha)=x\left(s, x_{0}\right)+\alpha\left[x\left(s, x_{0}+\varepsilon y_{0}\right)-x\left(s, x_{0}\right)\right]
$$

is determined by the fundamental theorem of calculus. Furthermore, by definition

$$
y\left(t, x_{0}, y_{0}\right)=y_{0}+\int_{0}^{t} J_{x} f\left(s, x\left(s, x_{0}\right)\right) y\left(s, x_{0}, y_{0}\right) d s .
$$

Therefore, if $0 \leq t \leq T_{1}$ one has

$$
\begin{aligned}
& \left|\frac{x\left(t, x_{0}+\varepsilon y_{0}\right)-x\left(t, x_{0}\right)}{\varepsilon}-y\left(t, x_{0}, y_{0}\right)\right| \\
& \leq \int_{0}^{t}\left|\left(\int_{0}^{1} J_{x} f\left(s, \xi_{\varepsilon}(s, \alpha)\right) d \alpha\right)\left[\frac{x\left(s, x_{0}+\varepsilon y_{0}\right)-x\left(s, x_{0}\right)}{\varepsilon}-y\left(s, x_{0}, y_{0}\right)\right]\right| d s \\
& \quad+\int_{0}^{t}\left|\left[\int_{0}^{1}\left(J_{x} f\left(s, \xi_{\varepsilon}(s, \alpha)\right)-J_{x} f\left(s, x\left(s, x_{0}\right)\right)\right) d \alpha\right] y\left(s, x_{0}, y_{0}\right)\right| d s
\end{aligned}
$$

Let us denote by $\eta_{\varepsilon}\left(t, x_{0}, y_{0}\right)$ the following integral

$$
\int_{0}^{t}\left|\left[\int_{0}^{1}\left(J_{x} f\left(s, \xi_{\varepsilon}(s, \alpha)\right)-J_{x} f\left(s, x\left(s, x_{0}\right)\right)\right) d \alpha\right] y\left(s, x_{0}, y_{0}\right)\right| d s .
$$

Next, we prove that $\eta_{\varepsilon}\left(t, x_{0}, y_{0}\right)$ goes to zero as $\varepsilon \rightarrow 0$ uniformly in $y_{0} \in B_{1}$ and $t \in\left[0, T_{1}\right]$. Firstly, let us fix $x_{0} \in \mathbb{R}^{N}$ and consider $j \in \mathbb{N}$ so that $\left\|x\left(\cdot, x_{0}\right)\right\|_{\infty}<j$ and also $\left\|\xi_{\varepsilon}(\cdot, \alpha)\right\|_{\infty}<j$ in $\left[0, T_{1}\right]$ for every $\varepsilon \leq 1$ and every $\alpha \in[0,1]$. From (3.19), one has

$$
\left|y\left(t, x_{0}, y_{0}\right)\right| \leq\left|y_{0}\right|+\int_{0}^{t} m^{j}(s)\left|y\left(s, x_{0}, y_{0}\right)\right| d s
$$

where, up to a rescaling due to the equivalence of the norms in $\mathbb{R}^{N \times N}, m^{j}(\cdot) \in L_{l o c}^{1}$ denotes the optimal $m$-bound for $J_{x} f$ on $B_{j}$. Therefore, using Grönwall Lemma one has

$$
\left|y\left(t, x_{0}, y_{0}\right)\right| \leq\left|y_{0}\right| \exp \left(\int_{0}^{t} m^{j}(s) d s\right) \leq\left|y_{0}\right| \exp \left(\int_{0}^{T_{1}} m^{j}(s) d s\right) .
$$

As a consequence, the solutions $y\left(\cdot, x_{0}, y_{0}\right)$ are uniformly bounded for $y_{0} \in B_{1}$ and $t \in\left[0, T_{1}\right]$. Moreover, notice that $\xi_{\varepsilon}(s, \alpha) \stackrel{\varepsilon \rightarrow 0}{\longrightarrow} x\left(s, x_{0}\right)$ uniformly in $\alpha \in[0,1]$, $y_{0} \in B_{1}$ and $s \in\left[0, T_{1}\right]$ and, since $J_{x} f \in \mathfrak{S C}_{p}\left(\mathbb{R}^{N \times N}\right)$, one also has that for almost every $s \in\left[0, T_{1}\right]$

$$
J_{x} f\left(s, \xi_{\varepsilon}(s, \alpha)\right) \stackrel{\varepsilon \rightarrow 0}{\longrightarrow} J_{x} f\left(s, x\left(s, x_{0}\right)\right)
$$

uniformly in $\alpha \in[0,1]$. Now, recalling that we have taken $j \in \mathbb{N}$ so that $\left\|x\left(\cdot, x_{0}\right)\right\|_{\infty}<j$ and also $\left\|\xi_{\varepsilon}(\cdot, \alpha)\right\|_{\infty}<j$ for every $\varepsilon \leq 1$ and every $\alpha \in[0,1]$, then we have that

$$
\left|\int_{0}^{1}\left(J_{x} f\left(s, \xi_{\varepsilon}(s, \alpha)\right)-J_{x} f\left(s, x\left(s, x_{0}\right)\right)\right) d \alpha\right| \leq 2 m^{j}(s)
$$


Therefore, applying Lebesgue theorem of dominated convergence one obtains that $\lim _{\varepsilon \rightarrow 0} \eta_{\varepsilon}\left(T_{1}, x_{0}, y_{0}\right)=0$ uniformly in $y_{0} \in B_{1}$, and recalling how $\eta_{\varepsilon}\left(\cdot, x_{0}, y_{0}\right)$ is defined in (3.21) one has

$$
\lim _{\varepsilon \rightarrow 0} \eta_{\varepsilon}\left(t, x_{0}, y_{0}\right)=0, \quad \text { uniformly in } y_{0} \in B_{1} \text { and } t \in\left[0, T_{1}\right]
$$

Now, reasoning as for (3.22), we have

$$
\int_{0}^{1}\left\|J_{x} f\left(s, \xi_{\varepsilon}(s, \alpha)\right)\right\| d \alpha \leq m^{j}(s)
$$

that, together with (3.20), provides

$$
\begin{aligned}
& \left|\frac{x\left(t, x_{0}+\varepsilon y_{0}\right)-x\left(t, x_{0}\right)}{\varepsilon}-y\left(t, x_{0}, y_{0}\right)\right| \\
& \quad \leq \eta_{\varepsilon}\left(t, x_{0}, y_{0}\right)+\int_{0}^{t} m^{j}(s)\left|\frac{x\left(s, x_{0}+\varepsilon y_{0}\right)-x\left(s, x_{0}\right)}{\varepsilon}-y\left(s, x_{0}, y_{0}\right)\right| d s,
\end{aligned}
$$

and applying Gronwall's inequality we get

$$
\begin{aligned}
& \left|\frac{x\left(t, x_{0}+\varepsilon y_{0}\right)-x\left(t, x_{0}\right)}{\varepsilon}-y\left(t, x_{0}, y_{0}\right)\right| \\
& \leq \eta_{\varepsilon}\left(t, x_{0}, y_{0}\right)+\int_{0}^{t} \eta_{\varepsilon}\left(s, x_{0}, y_{0}\right) m^{j}(s) \exp \left(\int_{s}^{t} m^{j}(r) d r\right) d s \\
& \leq \eta_{\varepsilon}\left(t, x_{0}, y_{0}\right)+c \int_{0}^{T_{1}} \eta_{\varepsilon}\left(s, x_{0}, y_{0}\right) m^{j}(s) d s
\end{aligned}
$$

where the positive constant $c$ satisfies $c \geq \exp \left(\int_{0}^{T_{1}} m^{j}(r) d r\right)$. Notice that, as $\varepsilon \rightarrow 0$, the right-hand side of (3.24) vanishes uniformly for $t \in\left[0, T_{1}\right]$ and $y_{0} \in B_{1}$, due to (3.23). As a consequence one has

$$
\lim _{\varepsilon \rightarrow 0^{+}} \frac{x\left(t, f, x_{0}+\varepsilon y_{0}\right)-x\left(t, f, x_{0}\right)}{\varepsilon}=y\left(t, f, J_{x} f, x_{0}, y_{0}\right),
$$

uniformly in $t \in\left[0, T_{1}\right]$ and $y_{0} \in B_{1}$, which ends the proof.

Remark 3.11. As follows, we provide conditions that allow to extend such conclusions to the solutions $x\left(\cdot, g, x_{0}\right)$ and $y\left(\cdot, g, G, x_{0}, y_{0}\right)$ of any pair of Cauchy Problems of the type

$$
\left\{\begin{array} { l } 
{ \dot { x } = g ( t , x ) } \\
{ x ( 0 ) = x _ { 0 } }
\end{array} \quad \text { and } \quad \left\{\begin{array}{l}
\dot{y}=G\left(t, x\left(t, g, x_{0}\right)\right) y \\
y(0)=y_{0}
\end{array}\right.\right.
$$

where $g \in \mathfrak{L} \mathfrak{C}$ and $G$ is either in $\Theta \mathfrak{C}$ or in $\mathfrak{W} \Theta \mathfrak{C}$ and in any case the pair $(g, G)$ is the limit, in a suitable topology, of a sequence $\left(f_{n}, J_{x} f_{n}\right)_{n \in \mathbb{N}}$ for which the variational equation is well-defined in the classical sense. Notice that $g$ may 
possibly not admit continuous partial derivatives with respect to $x$ and thus $G$ is possibly not the Jacobian of $g$ with respect to the variable $x$. However, we will still prove that

$$
\frac{\partial x\left(t, g, x_{0}\right)}{\partial x_{0}} \cdot y_{0}=y\left(t, g, G, x_{0}, y_{0}\right)
$$

for all $t$ in the maximal interval of definition $I_{g, x_{0}}$ of $x\left(t, g, x_{0}\right)$ and $y\left(t, g, G, x_{0}, y_{0}\right)$.

Theorem 3.12. Consider $E_{1} \subset \mathfrak{L C}_{p}$ with $L_{\text {loc }}^{1}$-equicontinuous m-bounds, assume that all the functions in $E_{1}$ are continuously differentiable with respect to $x$ for a.e. $t \in \mathbb{R}$ and, for any $f \in E_{1}$, assume that $J_{x} f \in \mathfrak{S C}_{p}\left(\mathbb{R}^{N \times N}\right)$. Let $\Theta$ be the suitable set of moduli of continuity given by the $m$-bounds of the functions in $E_{1}$ as in Definition 3.2, and consider

$$
E=\operatorname{cls}_{\left(\mathfrak{L C}_{p} \times \Theta \mathfrak{C}_{p}, \mathcal{T}_{\Theta} \times \mathcal{T}_{\Theta}\right)}\left\{\left(f, J_{x} f\right) \mid f \in E_{1}\right\} .
$$

For any $(g, G) \in E$, and following the notation of Remark 3.11, if $x\left(t, g, x_{0}\right)$ and $y\left(t, g, G, x_{0}, y_{0}\right)$ are the solutions of the Cauchy Problems in (3.26) defined for $t \in\left[T_{0}, T_{1}\right] \subset I_{g, x_{0}}$, then we have that

$$
\lim _{\varepsilon \rightarrow 0^{+}}\left|\frac{x\left(t, g, x_{0}+\varepsilon y_{0}\right)-x\left(t, g, x_{0}\right)}{\varepsilon}-y\left(t, g, G, x_{0}, y_{0}\right)\right|=0,
$$

uniformly for $t \in\left[T_{0}, T_{1}\right]$ and $y_{0} \in B_{1}$.

Proof. For any $f \in E_{1}$ we have that (3.27) holds with $g=f$ and $G=J_{x} f$ thanks to Theorem 3.10.

Now, consider $(g, G) \in E$ and let $\left(f_{n}\right)_{n \in \mathbb{N}}$ be a sequence of functions in $E_{1}$ such that $\left(f_{n}, J_{x} f_{n}\right)_{n \in \mathbb{N}}$ converges to $(g, G)$ in $\left(\mathfrak{L C}_{p} \times \Theta \mathfrak{C}_{p}\left(\mathbb{R}^{N \times N}\right), \mathcal{T}_{\Theta} \times \mathcal{T}_{\Theta}\right)$. From (3.25), we have that for any $n \in \mathbb{N}$, and for any $x_{1}, x_{2} \in \mathbb{R}^{N}$ such that $\left|x_{1}-x_{2}\right| \leq 1$, the following equality holds for every $t \in I_{f_{n}, x_{1}} \cap I_{f_{n}, x_{2}}$

$$
x\left(t, f_{n}, x_{1}\right)-x\left(t, f_{n}, x_{2}\right)=\int_{0}^{1} y\left(t, f_{n}, J_{x} f_{n}, \alpha x_{1}+(1-\alpha) x_{2}, x_{1}-x_{2}\right) d \alpha .
$$

Then, if $\left[T_{0}, T_{1}\right] \subset I_{g, x_{0}}$, thanks to Theorem 3.6, we have that the application

$$
\left[T_{0}, T_{1}\right] \times C \times B \rightarrow \mathbb{R}^{N} \times \mathbb{R}^{N}, \quad(t, h, H, \widehat{x}, \widehat{y}) \mapsto(x(t, h, \widehat{x}), y(t, h, H, \widehat{x}, \widehat{y})),
$$

is uniformly continuous and bounded, where

$$
\begin{aligned}
& C=\left\{\left(f_{n}, J_{x} f_{n}\right) \mid n \in \mathbb{N}\right\} \cup\{(g, G)\}, \\
& B=\left\{(\widehat{x}, \widehat{y}) \in \mathbb{R}^{N} \times \mathbb{R}^{N}|| \widehat{x}-x_{0} \mid \leq 1, \widehat{y} \in B_{1}\right\} .
\end{aligned}
$$

Thus, we have that, as $n \rightarrow \infty$, equation (3.28) becomes

$$
x\left(t, g, x_{1}\right)-x\left(t, g, x_{2}\right)=\int_{0}^{1} y\left(t, g, G, \alpha x_{1}+(1-\alpha) x_{2}, x_{1}-x_{2}\right) d \alpha .
$$


Eventually, if in (3.30) we consider $x_{2}=x_{0}$ and $x_{1}=x_{0}+\varepsilon y_{0}$, where $\varepsilon \leq 1$ and $y_{0} \in B_{1}$, and noticing that, due to the linearity of $y\left(t, g, G, x_{0}+\alpha \varepsilon y_{0}, y_{0}\right)$ with respect to the initial data $y_{0}$

$$
\frac{1}{\varepsilon} y\left(t, g, G, x_{0}+\alpha \varepsilon y_{0}, \varepsilon y_{0}\right)=y\left(t, g, G, x_{0}+\alpha \varepsilon y_{0}, y_{0}\right),
$$

then, we deduce that

$$
\begin{aligned}
\mid \frac{x\left(t, g, x_{0}+\varepsilon y_{0}\right)}{\varepsilon}-x\left(t, g, x_{0}\right) & -y\left(t, g, G, x_{0}, y_{0}\right) \mid \\
& =\left|\frac{1}{\varepsilon} \int_{0}^{1} y\left(t, g, G, x_{0}+\alpha \varepsilon y_{0}, \varepsilon y_{0}\right) d \alpha-y\left(t, g, G, x_{0}, y_{0}\right)\right| \\
& \leq \int_{0}^{1}\left|y\left(t, g, G, x_{0}+\alpha \varepsilon y_{0}, y_{0}\right)-y\left(t, g, G, x_{0}, y_{0}\right)\right| d \alpha .
\end{aligned}
$$

Then, applying Theorem 3.6 once again, when $\varepsilon \rightarrow 0$, and reasoning as before, we obtain the thesis.

As follows, we present an analogous result for the weak topology $\sigma_{\Theta}$ used in Theorem 3.4. However, a stricter assumption on the $m$-bounds of the Jacobian of $f$ has to be assumed.

Theorem 3.13. Consider $E_{1} \subset \mathfrak{L} \mathfrak{C}$ with $L_{\text {loc }}^{1}$-equicontinuous m-bounds, assume that all the functions in $E_{1}$ are continuously differentiable with respect to $x$ for a.e. $t \in \mathbb{R}$ and, assume that $\left\{J_{x} f \mid f \in E_{1}\right\} \subset \mathfrak{S} \mathfrak{C}\left(\mathbb{R}^{N \times N}\right)$ has $L_{\text {loc }}^{1}$-equicontinuous $m$-bounds. Let $\Theta$ be the suitable set of moduli of continuity given by the $m$-bounds of the functions in $E_{1}$ as in Definition 3.2 and consider

$$
E=\operatorname{cls}_{\left(\mathfrak{L} \mathfrak{C} \times \mathfrak{W} \Theta \mathfrak{C}, \sigma_{\Theta} \times \sigma_{\Theta}\right)}\left\{\left(f, J_{x} f\right) \mid f \in E_{1}\right\} .
$$

For any $(g, G) \in E$, and following the notation of Remark 3.11, if $x\left(t, g, x_{0}\right)$ and $y\left(t, g, G, x_{0}, y_{0}\right)$ are the solutions of the Cauchy Problems in (3.26) defined for $t \in\left[T_{0}, T_{1}\right] \subset I_{g, x_{0}}$, then we have that

$$
\lim _{\varepsilon \rightarrow 0^{+}}\left|\frac{x\left(t, g, x_{0}+\varepsilon y_{0}\right)-x\left(t, g, x_{0}\right)}{\varepsilon}-y\left(t, g, G, x_{0}, y_{0}\right)\right|=0,
$$

uniformly for $t \in\left[T_{0}, T_{1}\right]$ and $y_{0} \in B_{1}$.

Proof. The proof follows the same arguments of the one of Theorem 3.12, except for the fact that now $(g, G) \in E$ is such that there exists a sequence $\left(f_{n}\right)_{n \in \mathbb{N}}$ in $E_{1}$ so that

$$
\left(f_{n}, J_{x} f_{n}\right) \stackrel{n \rightarrow \infty}{\longrightarrow}(g, G) \text { in }\left(\mathfrak{L C} \times \mathfrak{W} \Theta \mathfrak{C}\left(\mathbb{R}^{N \times N}\right), \sigma_{\Theta} \times \sigma_{\Theta}\right) .
$$

Then, because of the $L_{l o c}^{1}$-equicontinuity of the $m$-bounds of $E_{1}$ and of $\left\{J_{x} f \mid\right.$ $\left.f \in E_{1}\right\}$, we can apply Theorem 3.4 and obtain that for any $\left[T_{0}, T_{1}\right] \subset I_{g, x_{0}}$, the application

$$
\left[T_{0}, T_{1}\right] \times C \times B \rightarrow \mathbb{R}^{N} \times \mathbb{R}^{N}, \quad(t, h, H, \widehat{x}, \widehat{y}) \mapsto(x(t, h, \widehat{x}), y(t, h, H, \widehat{x}, \widehat{y})),
$$


is uniformly continuous and bounded, where $C$ and $B$ are the sets in (3.29).

The rest of the proof is the same as the one of Theorem 3.12.

As a consequence of the previous theorems, it makes sense to name linearized and $\sigma$-linearized the skew-product flows obtained in Theorem 3.7(ii) and Theorem 3.5(ii), respectively, when $f$ is continuously differentiable with respect to $x$ for a.e. $t \in \mathbb{R}, F=J_{x} f$, and $h=0$. Particularly, we give the following definition.

Definition 3.14 (Linearized and $\sigma$-linearized skew-product flows). Let $f \in \mathfrak{L} \mathfrak{C}$ be continuously differentiable with respect to $x$ for a.e. $t \in \mathbb{R}$ and with $L_{\text {loc }}^{1}$ equicontinuous $m$-bounds. Let $\Theta$ be defined as in Remark 3.3 and denote by $J_{x} f \in \mathfrak{S} \mathfrak{C}\left(\mathbb{R}^{N \times N}\right)$ the Jacobian of $f$ with respect to the coordinates $x$.

- If $\mathbb{H}_{\mathcal{T}}=\operatorname{Hull}_{\left(\mathfrak{L C} \times \Theta \mathfrak{C}, \mathcal{T}_{\Theta} \times \mathcal{T}_{\Theta}\right)}\left(f, J_{x} f\right)$ and $\mathcal{U}_{\mathcal{T}}$ is the subset of $\mathbb{R} \times \mathbb{H}_{\mathcal{T}} \times \mathbb{R}^{N} \times \mathbb{R}^{N}$ given by

$$
\mathcal{U}_{\mathcal{T}}=\bigcup_{\substack{(g, G) \in \mathbb{H}_{\mathcal{T}} \\ x_{0} \in \mathbb{R}^{N}}}\left\{\left(t, g, G, x_{0}, y_{0}\right) \mid t \in I_{g, x_{0}}, y_{0} \in \mathbb{R}^{N}\right\}
$$

then we call a linearized skew-product flow the map

$$
\begin{aligned}
\Psi_{\mathcal{T}}: \mathcal{U}_{\mathcal{T}} \subset \mathbb{R} \times \mathbb{H}_{\mathcal{T}} \times \mathbb{R}^{N} \times \mathbb{R}^{N} & \rightarrow \quad \mathbb{H}_{\mathcal{T}} \times \mathbb{R}^{N} \times \mathbb{R}^{N} \\
\left(t, g, G, x_{0}, y_{0}\right) & \mapsto\left(g_{t}, G_{t}, x\left(t, g, x_{0}\right), y\left(t, g, G, x_{0}, y_{0}\right)\right),
\end{aligned}
$$

- If $\mathbb{H}_{\sigma}=\operatorname{Hull}_{\left(\mathfrak{L} \mathfrak{C} \times \mathfrak{W} \Theta \mathfrak{C}, \sigma_{\Theta} \times \sigma_{\Theta}\right)}\left(f, J_{x} f\right)$, where $J_{x} f$ has $L_{\text {loc }}^{1}$-equicontinuous $m$ bounds, and if $\mathcal{U}_{\sigma}$ is the subset of $\mathbb{R} \times \mathbb{H}_{\sigma} \times \mathbb{R}^{N} \times \mathbb{R}^{N}$ given by

$$
\mathcal{U}_{\sigma}=\bigcup_{\substack{(g, G) \in \mathbb{H} \sigma \\ x_{0} \in \mathbb{R}^{N}}}\left\{\left(t, g, G, x_{0}, y_{0}\right) \mid t \in I_{g, x_{0}}, y_{0} \in \mathbb{R}^{N}\right\},
$$

then, we call a $\sigma$-linearized skew-product flow the map

$$
\begin{aligned}
\Psi_{\sigma}: \mathcal{U}_{\sigma} \subset \mathbb{R} \times \mathbb{H}_{\sigma} \times \mathbb{R}^{N} \times \mathbb{R}^{N} & \rightarrow \quad \mathbb{H}_{\sigma} \times \mathbb{R}^{N} \times \mathbb{R}^{N} \\
\left(t, g, G, x_{0}, y_{0}\right) & \mapsto\left(g_{t}, G_{t}, x\left(t, g, x_{0}\right), y\left(t, g, G, x_{0}, y_{0}\right)\right),
\end{aligned}
$$

Next we give a simple example, for $N=1$, exhibiting the phenomenon cited in Remark 3.11. That is a limit problem for which the variational equation is not defined but, thanks to the continuity of the skew-product flow, it is still possible to obtain the differentiability of the solutions with respect to initial data.

Example 3.15. Consider the continuous function $H: \mathbb{R} \rightarrow \mathbb{R}$ such that $H(t)=0$ if $t<0$ and, for any $n \in \mathbb{N}, H(t)$ is defined in the interval [4n, $4 n+4]$ as follows:

$$
H(t)= \begin{cases}(1+n)(t-4 n), & \text { if } t \in I_{n}^{1}=\left[4 n, 4 n+\frac{1}{n+1}\right], \\ 1, & \text { if } t \in I_{n}^{2}=\left[4 n+\frac{1}{n+1}, 4 n+2-\frac{1}{n+1}\right], \\ -(1+n)(t-4 n-2), & \text { if } t \in I_{n}^{3}=\left[4 n+2-\frac{1}{n+1}, 4 n+2+\frac{1}{n+1}\right], \\ -1, & \text { if } t \in I_{n}^{4}=\left[4 n+2+\frac{1}{n+1}, 4 n+4-\frac{1}{n+1}\right], \\ (1+n)(t-4 n-4), & \text { if } t \in I_{n}^{5}=\left[4 n+4-\frac{1}{n+1}, 4 n+4\right] .\end{cases}
$$




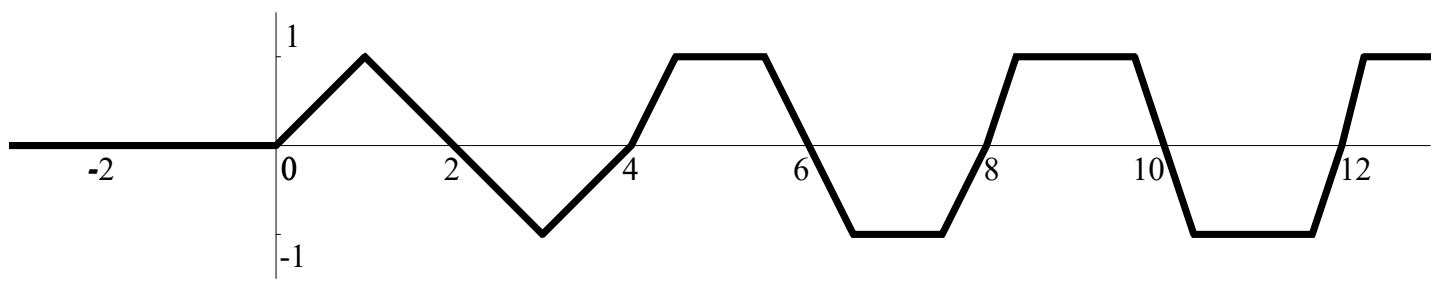

Figure 3.1: The function $H(t)$.

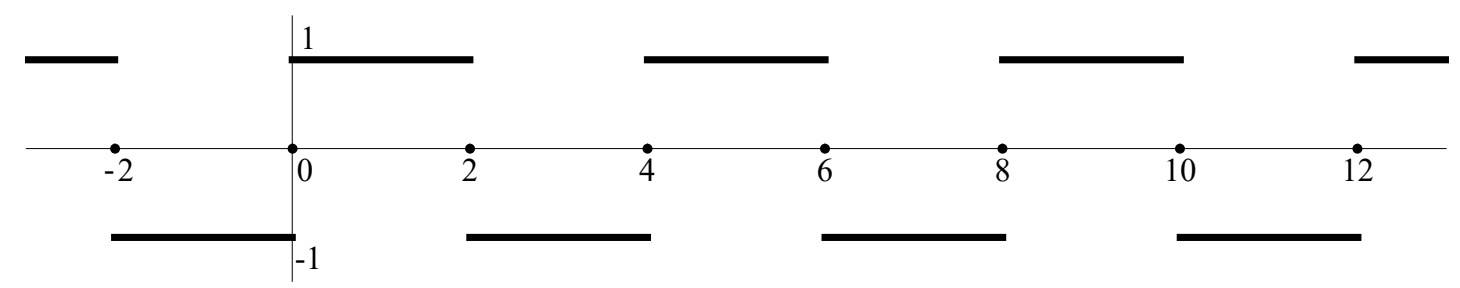

Figure 3.2: The function $\bar{H}(t)$.

Notice that, by construction (see also Figure 3.1), one has that for any $n \in \mathbb{N}$

$$
\int_{4 n}^{4 n+4} H(s) d s=0,
$$

and as $n \rightarrow \infty$ the measures of $I_{n}^{1}, I_{n}^{3}$ and $I_{n}^{5}$ go to zero, whereas the measures of $I_{n}^{2}$ and $I_{n}^{4}$ go to 2 . Thus, if we consider the sequence of translations of $H$ given by $\left(H_{4 k}(\cdot)\right)_{k \in \mathbb{N}}$, we have that

$$
H_{4 k}(t) \stackrel{k \rightarrow \infty}{\longrightarrow} \bar{H}(t)=\left\{\begin{array}{ll}
0, & \text { if } t=4 n, \\
1, & \text { if } t \in(4 n, 4 n+2), \\
0, & \text { if } t=4 n+2, \\
-1, & \text { if } t \in(4 n+2,4 n+4),
\end{array} \quad n \in \mathbb{Z}, \forall t \in \mathbb{R} .\right.
$$

Notice that $\bar{H}(\cdot)$ is a Borel function because of being a pointwise limit of continuous functions. Now consider the function $h: \mathbb{R} \rightarrow \mathbb{R}$ defined by

$$
h(t)=\int_{0}^{t} H(s) d s .
$$

Clearly $h \in C^{1}(\mathbb{R})$ since for all $t \in \mathbb{R}$ we have that $h^{\prime}(t)=H(t)$ and thus we also have that $\left|h^{\prime}(t)\right| \leq 1$; consequently, $h$ has Lipschitz constant equal to 1 . Moreover, $|h(t)| \leq 2$ for any $t \in \mathbb{R}$; indeed, thanks to (3.31), for each $t \in \mathbb{R}$ there exists $n_{0} \in \mathbb{N}$ such that

$$
|h(t)|=\left|\int_{4 n_{0}}^{t} H(s) d s\right| \leq 2
$$

Therefore, $\operatorname{cls}\left\{h_{\tau}(\cdot) \mid \tau \in \mathbb{R}\right\}$ is compact in $C(\mathbb{R})$ endowed with the compact open topology and $\left(h_{4 k}(\cdot)\right)_{k \in \mathbb{N}}$ converges uniformly on compact sets, up to a 
subsequence, to some bounded and Lipschitz function $\bar{h}$. Notice that

$$
\begin{aligned}
h_{4 k}(t)=h(t+4 k) & =\int_{0}^{t+4 k} H(s) d s=\int_{-4 k}^{t} H(u+4 k) d u \\
& =\int_{-4 k}^{0} H_{4 k}(u) d u+\int_{0}^{t} H_{4 k}(u) d u=\int_{0}^{t} H_{4 k}(u) d u,
\end{aligned}
$$

where the last equality is due to (3.31). Therefore, if in the previous chain of equalities we pass to the limit as $k \rightarrow \infty$ and use Lebesgue Theorem of dominated convergence, we have that

$$
\bar{h}(t)=\int_{0}^{t} \bar{H}(s) d s .
$$

Now, consider the functions $f \in \mathfrak{L C}_{p}$ and $F \in \mathfrak{S C}_{p}$ defined by

$$
f(t, x)=h\left(t+\frac{x}{3}\right) \quad \text { and } \quad F(t, x)=\frac{1}{3} H\left(t+\frac{x}{3}\right) .
$$

Since we already showed that for all $t \in \mathbb{R}$ one has $|h(t)| \leq 2$ and $\left|h^{\prime}(t)\right| \leq 1$, then we have that $f$ has a bounded $m$-bound, $m=2$, and a bounded $l$-bound, $l=1$, and they hold for all $x \in \mathbb{R}$. As a first consequence, from Remark 3.3, we have the common modulus of continuity $\theta(t)=2 t$. Then, consider $\operatorname{Hull}_{\left(\mathfrak{L C}_{p} \times \Theta \mathfrak{C}, \mathcal{T}_{\theta} \times \mathcal{T}_{\theta}\right)}(f, F)$, where, according to the notation used since the beginning of the section, we may write $F=J_{x} f$.

Now, let us consider the following family of differential systems whose vector fields are in the $\operatorname{Hull}_{\left(\mathfrak{L C}_{p} \times \Theta \mathfrak{C}_{p}, \mathcal{T}_{\theta} \times \mathcal{T}_{\theta}\right)}(f, F)$,

$$
\left\{\begin{array}{l}
\dot{x}=f_{4 k}(t, x) \\
\dot{y}=F_{4 k}(t, x(t)) y
\end{array} \quad k \in \mathbb{N} .\right.
$$

One can easily check that, for any $k \in \mathbb{N}$, the second differential equation in (3.32) is the variational equation of the first one, evaluated along the solution $x(t)$ of the first equation. Moreover, since $h_{4 k}(t) \stackrel{k \rightarrow \infty}{\longrightarrow} \bar{h}(t)$ uniformly on compact sets, then $f_{4 k}(t, x) \stackrel{k \rightarrow \infty}{\longrightarrow} g(t, x)$ in $\mathcal{T}_{D}$, where $g(t, x)=\bar{h}(t+x / 3)$. Actually, since $f$ has a bounded $l$-bound, then it satisfies the hypothesis of Corollary 2.32, and thus the convergence $f_{4 k}(t, x) \rightarrow \bar{g}(t, x)$ holds for any of the considered topologies and in particular in $\mathcal{T}_{\theta}$. Furthermore, we claim that $F_{4 k} \rightarrow G$ in $\mathcal{T}_{\theta}$, where $G(t, x)=(1 / 3) \bar{H}(t+x / 3)$. Indeed, for any compact interval $I \subset \mathbb{R}$, any $j \in \mathbb{N}$, and any $z(\cdot) \in C\left(I, B_{j}\right)$ with $\theta(t)=2 t$ as modulus of continuity, we have

$$
\begin{aligned}
\int_{I} \mid F_{4 k}( & t, z(t))-\left.G(t, z(t))\right|^{p} d t \\
& =\frac{1}{3^{p}} \int_{I}\left|H_{4 k}\left(t+\frac{z(t)}{3}\right)-\bar{H}\left(t+\frac{z(t)}{3}\right)\right|^{p} d t \\
& \leq 3^{1-p} \int_{I}\left|H_{4 k}\left(t+\frac{z(t)}{3}\right)-\bar{H}\left(t+\frac{z(t)}{3}\right)\right|^{p}\left|1+\frac{z^{\prime}(t)}{3}\right| d t \\
& \leq \frac{1}{3^{p}} \int_{I+[-j / 3, j / 3]}\left|H_{4 k}(s)-\bar{H}(s)\right|^{p} d s,
\end{aligned}
$$


where, in the first inequality, $z^{\prime}(t)$ is the derivative almost everywhere of $z(t)$, whose existence is granted by the fact that $z(t)$ is Lipschitz, and we use the fact that $1 / 3 \leq\left|1+z^{\prime}(t) / 3\right|$ for almost every $t \in I$. Moreover, the theorem of change of variables for the measurable case (see [31, Corollary 20.5, p.344]) has been used in the last inequality. Therefore, since $\left(H_{4 k}(s)\right)_{k \in \mathbb{N}}$ converges almost everywhere to $\bar{H}(s)$, the Lebesgue theorem of dominated convergence gives us the result. Finally, notice that $G(t, x)$ is a Borel function. Hence, thanks to Theorem 2.6, we have that $G \in \Theta \mathfrak{C}_{p}$.

In order to conclude that we are in front of a non trivial case of linearized skew product flow, we only need to check that $G \notin \mathfrak{S C}_{p}$, that is, $G$ does not satisfy (S), and $G$ does not coincide almost everywhere with a function in $\mathfrak{S C}_{p}$. Firstly, notice that for each fixed $t \in \mathbb{R}$ there is a numerable quantity of discontinuities in the variable $x$. In fact, given $x_{0} \in \mathbb{R}$ such that $t+x_{0} / 3=4 n$ for some $n \in \mathbb{N}$, we have that

$$
\lim _{x \rightarrow x_{0}^{-}} G(t, x)=\frac{1}{3} \quad \text { and } \quad \lim _{x \rightarrow x_{0}^{+}} G(t, x)=-\frac{1}{3} .
$$

Therefore, for each $t \in \mathbb{R}$ the function $G$ is not continuous in the variable $x$ and thus in particular it does not satisfy $(\mathbf{S})$. Furthermore, the set

$$
\left\{(t, x) \in \mathbb{R}^{2} \mid \lim _{z \rightarrow x^{-}} G(t, z) \neq \lim _{z \rightarrow x^{+}} G(t, z)\right\}
$$

is not negligible and thus it is not possible to change $G$ in a subset of $\mathbb{R}^{2}$ of measure zero so that it coincides with a function in $\mathfrak{S C}_{p}$.

\subsection{Exponential dichotomy and dichotomy spec- trum}

In this section we look more deeply into the properties of the linearized skewproduct flows introduced at the end of last section. In particular, we investigate the behavior of the solutions of the linear system when it has exponential dichotomy and study its dichotomy spectrum. Firstly, let us state some assumptions and simplify the notation.

Let $\mathbb{H}$ be either $\mathbb{H}_{\mathcal{T}}$ or $\mathbb{H}_{\sigma}$ as defined in Definition 3.14, and assume that for each $(g, G) \in \mathbb{H}$. the solutions of

$$
\begin{cases}\dot{x}=g(t, x), & x(0)=x_{0}, \\ \dot{y}=G(t, x) y, & y(0)=y_{0},\end{cases}
$$

are globally defined or, equivalently, $x\left(\cdot, g, x_{0}\right)$ is globally defined. As a consequence, the linearized skew-product flow is defined on the whole $\mathbb{R} \times \mathbb{H} \times \mathbb{R}^{N} \times \mathbb{R}^{N}$. 
Moreover, denoting by $\Omega=\mathbb{H} \times \mathbb{R}^{N}$, any of the continuous skew-product flows in Definition 3.14 can be read as a continuous linear skew-product flow

$$
\begin{aligned}
\Psi: \mathbb{R} \times \Omega \times \mathbb{R}^{N} & \rightarrow \quad \Omega \times \mathbb{R}^{N} \\
\left(t, \omega, y_{0}\right) & \mapsto\left(\omega_{t}, y\left(t, \omega, y_{0}\right)\right),
\end{aligned}
$$

where the flow on the base $\mathbb{R} \times \Omega \rightarrow \Omega,(t, \omega) \mapsto \omega_{t}$ is defined, for each $\omega=$ $\left(g, G, x_{0}\right)$, by $\omega_{t}=\left(g_{t}, G_{t}, x\left(t, g, x_{0}\right)\right)$. Additionally, consider the function $A$ : $\Omega \rightarrow \mathbb{R}^{N \times N}$ defined as follows

$$
A(\omega)= \begin{cases}\lim _{h \rightarrow 0} \frac{1}{h} \int_{0}^{h} G\left(s, x\left(s, g, x_{0}\right)\right) d s & \text { if the limit exists } \\ 0 & \text { otherwise. }\end{cases}
$$

Notice that, in fact,

$$
A\left(\omega_{t}\right)=G\left(t, x\left(t, g, x_{0}\right)\right) \quad \text { for a.e. } t \in \mathbb{R} .
$$

Indeed, fixed $\omega_{t}=\left(g_{t}, G_{t}, x\left(t, g, x_{0}\right)\right)$, one has

$$
\begin{aligned}
\lim _{h \rightarrow 0} & \frac{1}{h} \int_{0}^{h} G_{t}\left(s, x\left(s, g_{t}, x\left(t, g, x_{0}\right)\right)\right) d s=\lim _{h \rightarrow 0} \frac{1}{h} \int_{0}^{h} G\left(s+t, x\left(s+t, g, x_{0}\right)\right) d s \\
=\lim _{h \rightarrow 0} \frac{1}{h} \int_{t}^{t+h} G\left(u, x\left(u, g, x_{0}\right)\right) d u & =G\left(t, x\left(t, g, x_{0}\right)\right) \quad \text { for a.e. } t \in \mathbb{R}
\end{aligned}
$$

where the last step in the previous chain of equalities is due to Corollary III.12.7 in [24], which implies (3.34).

Then, the family of systems $\dot{y}=G\left(t, x\left(t, g, x_{0}\right)\right) y$, with $\omega=\left(g, G, x_{0}\right) \in \Omega$, can be written as follows

$$
\dot{y}=A\left(\omega_{t}\right) y, \quad \omega \in \Omega .
$$

Thus, if $\Phi(t, \omega)$ denotes the fundamental matrix solution of the system corresponding to $\omega$ with $\Phi(0, \omega)=\mathrm{I}_{N}$, we have that $y\left(t, \omega, y_{0}\right)=\Phi(t, \omega) y_{0}$.

Let us recall that the linear skew-product flow (3.33), or that the family (3.35), has exponential dichotomy on $I$ over the set $\Delta$, if the linear skew-product flow (3.33) satisfies the conditions given in Definition 1.14, i.e. there are a continuous family of projections $P: \Delta \rightarrow \mathcal{L}\left(\mathbb{R}^{N}, \mathbb{R}^{N}\right), \omega \mapsto P(\omega)$, and constants $K \geq 1$ and $\alpha>0$, such that for every $s, t \in I$ and every $\omega \in \Delta$ one has

$$
\begin{array}{ll}
\left\|\Phi(t, \omega) P(\omega) \Phi^{-1}(s, \omega)\right\| \leq K e^{-\alpha(t-s)} & \text { if } t \geq s, \\
\left\|\Phi(t, \omega)\left(\mathrm{I}_{N}-P(\omega)\right) \Phi^{-1}(s, \omega)\right\| \leq K e^{\alpha(t-s)} & \text { if } t \leq s .
\end{array}
$$

In particular, when $\Delta$ reduces to a point $\omega=\left(f, G, x_{0}\right)$, it is said that the corresponding system $\dot{y}=A\left(\omega_{t}\right) y$, i.e. $\dot{y}=G\left(t, x\left(t, g, x_{0}\right)\right.$ y has exponential dichotomy on $I$. 
Definition 3.16. The set $\mathbb{A}(\omega)$ will denote the alpha limit set of a point $\omega=$ $\left(g, G, x_{0}\right) \in \Omega$, that is, $\widehat{\omega}=\left(\widehat{g}, \widehat{G}, \widehat{x}_{0}\right) \in \mathbb{A}(\omega)$ if there is a sequence $\left(t_{n}\right)_{n \in \mathbb{N}}$ in $\mathbb{R}$ such that $t_{n} \downarrow-\infty$ and $\widehat{\omega}=\lim _{n \rightarrow \infty} \omega_{t_{n}}$ in the corresponding product topology, i.e. $\left(\widehat{g}, \widehat{G}, \widehat{x}_{0}\right)=\lim _{n \rightarrow \infty}\left(g_{t_{n}}, G_{t_{n}}, x\left(t_{n}, g, x_{0}\right)\right)$.

Analogously $\widehat{\omega}=\left(\widehat{g}, \widehat{G}, \widehat{x}_{0}\right)$ belongs to the omega limit set $\mathbb{O}(\omega)$ if there is a sequence $\left(t_{n}\right)_{n \in \mathbb{N}}$ in $\mathbb{R}$ such that $t_{n} \uparrow \infty$ and $\widehat{\omega}=\lim _{n \rightarrow \infty} \omega_{t_{n}}$, i.e. $\left(\widehat{g}, \widehat{G}, \widehat{x}_{0}\right)=$ $\lim _{n \rightarrow \infty}\left(g_{t_{n}}, G_{t_{n}}, x\left(t_{n}, g, x_{0}\right)\right)$. Finally, $\mathbb{H}(\omega)$ will denote the closure in $\Omega$ of the set $\left\{\omega_{t}=\left(g_{t}, G_{t}, x\left(t, g, x_{0}\right)\right) \mid t \in \mathbb{R}\right\}$ for the corresponding product topology on $\Omega$.

The next result shows how the exponential dichotomy of a particular system can be transferred to the exponential dichotomy of the skew-product flow over its alpha limit set, its omega limit set or its hull.

Proposition 3.17. Let $\omega=\left(g, G, x_{0}\right) \in \Omega$.

(i) If the linear system $\dot{y}=A\left(\omega_{t}\right)$ y has exponential dichotomy on $(-\infty, 0]$, then the skew-product flow (3.33) has exponential dichotomy over the alpha limit set $\mathbb{A}(\omega) \subset \Omega$.

(ii) If the linear system $\dot{y}=A\left(\omega_{t}\right)$ y has exponential dichotomy on $[0, \infty)$, then the skew-product flow (3.33) has exponential dichotomy over the omega limit set $\mathbb{O}(\omega) \subset \Omega$.

(iii) If the linear system $\dot{y}=A\left(\omega_{t}\right)$ y has exponential dichotomy, then the skewproduct flow (3.33) has exponential dichotomy over the hull $\mathbb{H}(\omega) \subset \Omega$.

Proof. (i) Let $P(\omega)$ be the projection corresponding to the exponential dichotomy on $(-\infty, 0]$ for the system $\dot{y}=A\left(\omega_{t}\right) y$ and define the family of projections

$$
P\left(\omega_{r}\right)=\Phi(r, \omega) P(\omega) \Phi^{-1}(r, \omega) \quad \text { for each } r \leq 0 .
$$

Then, one has

$$
\begin{aligned}
& \left\|\Phi\left(t, \omega_{r}\right) P\left(\omega_{r}\right) \Phi^{-1}\left(s, \omega_{r}\right)\right\|=\left\|\Phi\left(t, \omega_{r}\right) \Phi(r, \omega) P(\omega) \Phi^{-1}(r, \omega) \Phi^{-1}\left(s, \omega_{r}\right)\right\| \\
& =\left\|\Phi(t+r, \omega) P(\omega) \Phi^{-1}(s+r, \omega)\right\| \quad \text { and } \\
& \begin{aligned}
\| \Phi(t, & \left.\omega_{r}\right)\left(\mathrm{I}_{N}-P\left(\omega_{r}\right)\right) \Phi^{-1}\left(s, \omega_{r}\right) \| \\
& =\left\|\Phi\left(t, \omega_{r}\right) \Phi^{-1}\left(s, \omega_{r}\right)-\Phi\left(t, \omega_{r}\right) \Phi(r, \omega) P(\omega) \Phi^{-1}(r, \omega) \Phi^{-1}\left(s, \omega_{r}\right)\right\| \\
& =\left\|\Phi\left(t, \omega_{r}\right) \Phi(r, \omega) \Phi^{-1}(r, \omega) \Phi^{-1}\left(s, \omega_{r}\right)-\Phi(t+r, \omega) P(\omega) \Phi^{-1}(s+r, \omega)\right\| \\
& =\left\|\Phi(t+r, \omega)\left(\mathrm{I}_{N}-P(\omega)\right) \Phi^{-1}(s+r, \omega)\right\| .
\end{aligned}
\end{aligned}
$$

Consequently, one has

$$
\begin{array}{ll}
\left\|\Phi\left(t, \omega_{r}\right) P\left(\omega_{r}\right) \Phi^{-1}\left(s, \omega_{r}\right)\right\| \leq K e^{-\alpha(t-s)} & \text { if } \quad s \leq t \leq-r \\
\left\|\Phi\left(t, \omega_{r}\right)\left(\mathrm{I}_{N}-P\left(\omega_{r}\right)\right) \Phi^{-1}\left(s, \omega_{r}\right)\right\| \leq K e^{\alpha(t-s)} & \text { if } \quad t \leq s \leq-r .
\end{array}
$$


Next we take $\widehat{\omega} \in \mathbb{A}(\omega)$ with $\widehat{\omega}=\lim _{n \rightarrow \infty} \omega_{r_{n}}$ for a sequence $r_{n} \downarrow-\infty$. From (3.37) and (3.36), we deduce that $\left\|P\left(\omega_{r}\right)\right\| \leq K$ for every $r \leq 0$ and hence, the sequence of projections $\left(P\left(\omega_{r_{n}}\right)\right)_{n \in \mathbb{N}}$ admits a subsequence converging to a projection $P(\widehat{\omega})$, whose uniqueness is guaranteed by Proposition 1.56 in [34]. From this fact, (3.38) and the continuity of the flow on the base $\Omega$, we deduce that $\dot{y}=A\left(\widehat{\omega}_{t}\right) y$ admits exponential dichotomy with projection $P(\widehat{\omega})$, that is

$$
\begin{array}{ll}
\left\|\Phi(t, \widehat{\omega}) P(\widehat{\omega}) \Phi^{-1}(s, \widehat{\omega})\right\| \leq K e^{-\alpha(t-s)} & \text { if } t \geq s \\
\left\|\Phi(t, \widehat{\omega})\left(\mathrm{I}_{N}-P(\widehat{\omega})\right) \Phi^{-1}(s, \widehat{\omega})\right\| \leq K e^{\alpha(t-s)} \quad \text { if } \quad t \leq s .
\end{array}
$$

In order to conclude the proof, we show the continuity of

$$
P: \mathbb{A}(\omega) \rightarrow \mathcal{L}\left(\mathbb{R}^{N}, \mathbb{R}^{N}\right), \quad \widehat{\omega} \mapsto P(\widehat{\omega})
$$

To the aim, consider a sequence $\left(\widehat{\omega}^{n}\right)_{n \in \mathbb{N}}$ in $\mathbb{A}(\omega)$ converging to some $\widehat{\omega} \in \mathbb{A}(\omega)$ and let us prove that $\left(P\left(\widehat{\omega}^{n}\right)\right)_{n \in \mathbb{N}}$ converges to $P(\widehat{\omega})$. As before, from (3.39), with $t=s$, one has that for all $n \in \mathbb{N}:\left\|P\left(\widehat{\omega}^{n}\right)\right\| \leq K$ and thus, it converges, up to a subsequence, to a projection $\widehat{P}$, and again, from (3.39) for each $n \in \mathbb{N}$, the continuity of the flow on the base $\Omega$ and the uniqueness of the projection, we have that $\widehat{P}=P(\widehat{\omega})$. Then, one has the exponential dichotomy of the skew-product flow (3.33) over $\mathbb{A}(\omega)$, as stated in (i).

The proofs of (ii) and (iii) are omitted because analogous.

Before proceeding, recall that taken $\Delta \subset \Omega$, the dichotomy spectrum of the linear skew-product flow (3.33) over $\Delta$, denoted by $\Sigma(\Delta)$, is the set of $\gamma \in \mathbb{R}$ such that the family $\dot{y}=\left(A\left(\theta_{t} \omega\right)-\gamma \mathrm{I}_{N}\right) y$ does not have exponential dichotomy over $\Delta$. As a consequence of the previous theorem we can relate the spectrum of $\omega \in \Omega$ to the spectrum of its hull.

Remark 3.18. Considered two subsets $\Delta_{1}$ and $\Delta_{2}$ of $\Omega$ such that $\Delta_{1} \subset \Delta_{2}$, and recalling Definition 1.15, one has that $\Sigma\left(\Delta_{1}\right) \subset \Sigma\left(\Delta_{2}\right)$. Therefore, since in particular $\{\omega\} \subset \mathbb{H}(\omega)$, we have that $\Sigma(\omega) \subset \Sigma(\mathbb{H}(\omega))$. However, by Proposition 3.17(iii), we actually have that

$$
\Sigma(\omega)=\Sigma(\mathbb{A}(\omega))=\Sigma(\mathbb{O}(\omega))=\Sigma(\mathbb{H}(\omega))
$$

which in turn implies that for any $\omega_{1} \in \mathbb{H}(\omega)$ one has

$$
\Sigma\left(\omega_{1}\right) \subset \Sigma(\mathbb{H}(\omega))=\Sigma(\omega)
$$

As we have already noticed in Section 1.2.2, Sacker and Sell [51] proved that, if $\Delta$ is an invariantly connected compact invariant set of $\Omega$, then $\Sigma(\Delta)$ is the union of $k$ compact intervals

$$
\Sigma(\Delta)=\left[a_{1}, b_{1}\right] \cup \cdots \cup\left[a_{k}, b_{k}\right]
$$


where $1 \leq k \leq N$ and $a_{1} \leq b_{1}<a_{2} \leq b_{2}<\cdots \leq a_{k} \leq b_{k}$.

However, from Proposition 3.17 we deduce that $\Sigma(\omega)=\Sigma(\mathbb{H}(\omega))$ but $\mathbb{H}(\omega)$ is not necessarily compact. Yet, we can look into the dichotomy spectrum introduced by Siegmund in [55] for a single system. In particular, due to Theorem 1.20, a linear system $\dot{y}=A\left(\omega_{t}\right)$ y keeps having a spectrum like $\Sigma(\omega)=$ $\left[a_{1}, b_{1}\right] \cup \cdots \cup\left[a_{k}, b_{k}\right]$ if and only if it has bounded growth, i.e. there exist constants $K \geq 1$ and $\alpha \geq 0$ such that

$$
\left\|\Phi(t, \omega) \Phi^{-1}(s, \omega)\right\| \leq K e^{\alpha|t-s|} \quad \text { for } \quad t, s \in \mathbb{R} .
$$

Moreover, in such a case the spectral manifolds $\mathcal{W}_{0}$ and $\mathcal{W}_{k+1}$ (see Theorem 1.19) are trivial, i.e. $\mathbb{R} \times \mathbb{R}^{N}=\mathcal{W}_{1} \oplus \cdots \oplus \mathcal{W}_{k}$.

We finish this section looking into conditions under which Carathéodory systems have bounded growth and thus the dichotomy spectrum $\Sigma(\omega)=\Sigma(\mathbb{H}(\omega))$ is a finite number of compact intervals as in the Sacker-Sell dichotomy spectrum.

Proposition 3.19. Let $\omega=\left(g, G, x_{0}\right) \in \Omega$ fixed. Assume that $G$ has $L_{l o c}^{1}$ bounded $m$-bounds and that $x\left(\cdot, g, x_{0}\right)$ is bounded. Then the system $\dot{y}=A\left(\omega_{t}\right) y$ has bounded growth and $\Sigma(\omega)=\left[a_{1}, b_{1}\right] \cup \cdots \cup\left[a_{k}, b_{k}\right]$.

Proof. Using the notation introduced in Theorem 1.2, let $I_{g, x_{0}}$ be the interval of definition of $x\left(\cdot, g, x_{0}\right)$ and let $j \in \mathbb{N}$ be such that $\left\|x\left(\cdot, g, x_{0}\right)\right\|_{L^{\infty}\left(I_{g, x_{0}}\right)} \leq j$. If $m^{j}$ is an $m$-bound of $G$ on $B_{j}$ satisfying the assumption of $L_{l o c}^{1}$-boundedness, then there is a positive constant $\alpha$ such that

$$
\sup _{s \in \mathbb{R}} \int_{0}^{1} m^{j}(r+s) d r \leq \alpha .
$$

Consider $s, t \in I_{g, x_{0}}$ and assume that $s \leq t$, the other case being analogous. Notice that $\Phi(t, \omega) \Phi^{-1}(s, \omega) y_{0}=y\left(t, \omega, s, y_{0}\right)$. Then

$$
\left|y\left(t, \omega, s, y_{0}\right)\right| \leq\left|y_{0}\right|+\int_{s}^{t}\left\|G\left(u, x\left(u, g, x_{0}\right)\right)\right\|\left|y\left(u, \omega, s, y_{0}\right)\right| d u
$$

and Gronwall inequality provides

$$
\begin{aligned}
\left|y\left(t, \omega, s, y_{0}\right)\right| & \leq\left|y_{0}\right| \exp \left(\int_{s}^{t}\left\|G\left(u, x\left(u, g, x_{0}\right)\right)\right\| d u\right) \\
& =\left|y_{0}\right| \exp \left(\int_{0}^{t-s}\left\|G_{s}\left(r, x\left(r+s, g, x_{0}\right)\right)\right\| d r\right) .
\end{aligned}
$$

Using the equivalence of the 2-norm and the matrix norm, and up to rescaling $m^{j}(\cdot)$ by a constant, we deduce that

$$
\| G_{s}\left(r, x\left(r+t, g, x_{0}\right) \| \leq m^{j}(r+s) .\right.
$$


Then, from (3.41), and using (3.40) on a finite covering of the interval $[0, t-s]$ with subintervals of unit length, we can conclude that

$$
\left|y\left(t, \omega, s, y_{0}\right)\right| \leq K e^{\alpha(t-s)}\left|y_{0}\right|
$$

for an appropriate constant $K \geq 1$, which ends the proof.

Recall that $\Omega$ is defined as either $\mathbb{H}_{\mathcal{T}} \times \mathbb{R}^{N}$ or $\mathbb{H}_{\sigma} \times \mathbb{R}^{N}$, where $\mathbb{H}_{\sigma}$ and $\mathbb{H}_{\mathcal{T}}$ are defined in Definition 3.14. Notice that in the case in which $\Omega=\mathbb{H}_{\mathcal{T}} \times \mathbb{R}^{N}$ both the assumptions of Proposition 3.19 are necessary, whereas if $\Omega=\mathbb{H}_{\sigma} \times \mathbb{R}^{N}$, then the $L_{l o c}^{1}$-boundedness for the $m$-bounds of $G$ is already implied by the $L_{l o c}^{1}$ equicontinuity for the $m$-bounds of $J_{x} f$ thanks to Proposition 2.29.

\subsection{More applications}

We conclude the chapter presenting a theoretical application of the continuity results contained in Section 3.1, and examples from control theory and compartmental dynamical systems.

\subsubsection{Existence of solutions for a differential problem in $\mathfrak{W} \Theta \mathfrak{C}$}

As follows we give a theorem of existence of the solutions for differential problems whose vector fields are in $\mathfrak{W} \Theta \mathfrak{C}$, i.e. not necessarily continuous in the space variables either. The underlying condition is that such vector fields are limit of sequences in $\mathfrak{S C}$ with $L_{\text {loc }}^{1}$-equicontinuous $m$-bounds in the topology $\sigma_{\Theta}$ (or any stronger topology of course), where $\Theta$ is the suitable set of moduli of continuity given by the $m$-bounds as in Definition 3.2. Notice that this approach has some similarities with the work on discontinuous autonomous systems by Dieci et al. [23].

Theorem 3.20. Let $\left(f_{n}\right)_{n \in \mathbb{N}}$ be a sequence in $\mathfrak{S C}$ with $L_{l o c}^{1}$-equicontinuous $m$ bounds and $\Theta$ be the suitable set of moduli of continuity given by the m-bounds as in Definition 3.2. Assume that $\left(f_{n}\right)_{n \in \mathbb{N}}$ converges to some $f$ in $\left(\mathfrak{W} \Theta \mathfrak{C}, \sigma_{\Theta}\right)$ and that $\left(x_{0, n}\right)_{n \in \mathbb{N}}$ is a sequence in $\mathbb{R}^{N}$ converging to $x_{0} \in \mathbb{R}^{N}$. Then, denoting by $x_{n}(\cdot)$ a solution, in the sense of Definition 1.1, for the differential problem $\dot{x}=f_{n}(t, x)$ defined on the maximal interval $\left(a_{n}, b_{n}\right)$ and such that $0 \in\left(a_{n}, b_{n}\right)$ and $x_{n}(0)=x_{0, n}$, we have

(i) $\limsup _{n \rightarrow \infty} a_{n}=a^{*}<0$, and $\liminf _{n \rightarrow \infty} b_{n}=b^{*}>0$.

(ii) There exist $a^{*}<a<b<b^{*}$ and a continuous function $x(\cdot)$ such that, up to a subsequence,

$$
x_{n}(\cdot) \stackrel{n \rightarrow \infty}{\longrightarrow} x(\cdot)
$$

uniformly on the compact subsets of $(a, b)$. 
(iii) For every $s, t \in(a, b)$, the function $x(\cdot)$ satisfies

$$
x(t)=x(s)+\int_{s}^{t} f(u, x(u)) d u .
$$

Proof. Firstly, we aim to prove that $b^{*}>0$. Assume, on the contrary, that $\liminf _{n \rightarrow \infty} b_{n}=0$, that is, up to a subsequence, $\left(b_{n}\right)_{n \in \mathbb{N}}$ converges to 0 . Moreover, consider the following constant

$$
0<\rho=1+\max \left\{\left|x_{0, n}\right| \mid n \in \mathbb{N}\right\},
$$

and, for every $n \in \mathbb{N}$, set

$$
T_{n}=\sup \left\{t \in\left[0, b_{n}\right)|| x_{n}(s) \mid \leq \rho \forall s \in[0, t]\right\} .
$$

Since, for every $n \in \mathbb{N}$, one has $0 \leq T_{n} \leq b_{n}$ by construction, and since $\left(b_{n}\right)_{n \in \mathbb{N}}$ converges to 0 by assumption, then one has that

$$
\lim _{n \rightarrow \infty} T_{n}=0 .
$$

Consider $j \in \mathbb{N}$ so that $\rho<j$ and let $\left(m_{n}(\cdot)\right)_{n \in \mathbb{N}}=\left(m_{f_{n}}^{j}(\cdot)\right)_{n \in \mathbb{N}}$ be the sequence of optimal $m$-bounds of $\left(f_{n}\right)_{n \in \mathbb{N}}$ on $B_{j}$. Fixed $0<\varepsilon<1$, by the $L_{l o c}^{1}$-equicontinuity of the $m$-bounds of the sequence $\left(f_{n}\right)_{n \in \mathbb{N}}$, there exists $\delta>0$ such that

$$
0 \leq t<\delta \quad \Rightarrow \quad \sup _{n \in \mathbb{N}} \int_{0}^{t} m_{n}^{j}(s) d s<\varepsilon
$$

However, by (3.44), one has that there exists $n_{0} \in N$ such that if $n>n_{0}$ then $T_{n}<\delta$ and thus, by definition of solution, and using (3.45) and (3.42), one has

$$
\left|x_{n}\left(T_{n}\right)\right| \leq\left|x_{n}(0)\right|+\int_{0}^{T_{n}} m_{n}^{j}(s) d s \leq \rho-1+\varepsilon<\rho,
$$

which is a contradiction due to the fact that for every $n \in \mathbb{N},\left|x_{n}\left(T_{n}\right)\right|=\rho$ thanks to the continuity of $x_{n}(\cdot)$ and (3.43). Therefore, one has that that $\liminf _{n \rightarrow \infty} b_{n}=$ $b *>0$. Analogous reasonings holds for $a^{*}$, which concludes part (i).

As regards (ii) and (iii), we prove the existence of $x(\cdot)$ in $[0, b)$. Analogous arguments leads to the result in the interval $(a, 0]$. Consider the constant $\rho$ defined in $(3.42)$, and define $z_{n}:[0, \infty) \rightarrow \mathbb{R}^{N}$ by

$$
z_{n}(t)= \begin{cases}x_{n}(t), & \text { if } 0 \leq t<T_{n} \\ x_{n}\left(T_{n}\right), & \text { if } T_{n}<+\infty \text { and } T_{n} \leq t\end{cases}
$$

where, for every $n \in \mathbb{N}, T_{n}$ is the one defined in (3.43). Notice that by (3.42) and by the continuity of the functions $x_{n}(\cdot), n \in \mathbb{N}$, we have that $T_{n}>0$ for any $n \in \mathbb{N}$. In particular, notice that $\left(z_{n}(\cdot)\right)_{n \in \mathbb{N}}$ is uniformly bounded. For the fixed $j \in \mathbb{N}$ 
such that $\rho<j$, and recalling that we denoted by $\left(m_{n}(\cdot)\right)_{n \in \mathbb{N}}=\left(m_{f_{n}}^{j}(\cdot)\right)_{n \in \mathbb{N}}$, the sequence of optimal $m$-bounds of $\left(f_{n}\right)_{n \in \mathbb{N}}$ on $B_{j}$, we have that, if $t_{1}, t_{2} \in\left[0, T_{n}\right)$, and $t_{1}<t_{2}$, then

$$
\left|z_{n}\left(t_{1}\right)-z_{n}\left(t_{2}\right)\right| \leq \int_{t_{1}}^{t_{2}}\left|f_{n}\left(s, z_{n}(s)\right)\right| d s \leq \int_{t_{1}}^{t_{2}} m_{n}(s) d s .
$$

Fixed $\varepsilon>0$, since $\left\{f_{n} \mid n \in \mathbb{N}\right\}$ admits $L_{\text {loc }}^{1}$-equicontinuous $m$-bounds, there exists $\delta=\delta(\varepsilon)>0$ such that, if $0 \leq t_{1} \leq t_{2}<T_{n}$, and $t_{2}-t_{1}<\delta$, then the right-hand side in (3.47) is smaller than $\varepsilon$. In fact, the inequality $\left|z_{n}\left(t_{1}\right)-z_{n}\left(t_{2}\right)\right|<\varepsilon$ is always true in $\left[0, b^{*}\right)$ whenever $t_{2}-t_{1}<\delta$ because in $\left[T_{n}, b^{*}\right)$ the difference on the lefthand side of (3.47) is zero. Thus, the sequence $\left(z_{n}(\cdot)\right)_{n \in \mathbb{N}}$ is equicontinuous. Then, Ascoli-Arzelá's theorem implies that, up to a subsequence, $\left(z_{n}(\cdot)\right)_{n \in \mathbb{N}}$ converges uniformly to some continuous function $x: I \rightarrow \mathbb{R}^{N}$ in any compact interval $I \subset\left[0, b^{*}\right)$. In this way it is possible to define $x(\cdot)$ on the whole $\left[0, b^{*}\right)$. Moreover, set

$$
b=\sup \left\{t \in\left[0, b^{*}\right)|| x(s) \mid<\rho-1 / 2 \quad \forall s \in[0, t]\right\},
$$

and notice that $b>0$ because $\left(x_{0, n}\right)_{n \in \mathbb{N}}$ converges to $x_{0}, x(\cdot)$ is continuous and (3.42) holds. Since $z_{n}(\cdot)$ converges uniformly to $x(\cdot)$ in any compact interval $\left[0, b_{1}\right]$, with $b_{1}<b$ then there exists $n_{0} \in \mathbb{N}$ such that if $n>n_{0}$, then

$$
\left|z_{n}(t)\right|<\rho-1 / 4 \quad \forall t \in\left[0, b_{1}\right] .
$$

Therefore, for any $t \in\left[0, b_{1}\right]$ and for any $n>n_{0}$ one has $z_{n}(t)=x_{n}(t)$ and thus

$$
z_{n}(t)=x_{0, n}+\int_{0}^{t} f_{n}\left(s, z_{n}(s)\right) d s, \quad t \in\left[0, b_{1}\right], n>n_{0} .
$$

Since we already know that $z_{n}(\cdot)$ converges uniformly to $x(\cdot)$ in $\left[0, b_{1}\right]$, if we prove that for any $t \in\left[0, b_{1}\right]$ one has

$$
\lim _{n \rightarrow \infty} \int_{0}^{t} f_{n}\left(s, z_{n}(s)\right) d s=\int_{0}^{t} f(s, x(s)) d s,
$$

then, passing to the limit as $n \rightarrow \infty$ in (3.48), we would have that $x(\cdot)$ is a solution of the limit problem $\dot{x}=f(t, x), x(0)=x_{0}$ in $\left[0, b_{1}\right]$. Let us fix $t \in\left[0, b_{1}\right] \cap \mathbb{Q}$ and consider the compact set $\mathcal{K}=\left\{z_{n}(\cdot) \mid n \in \mathbb{N}\right\} \cup\{x(\cdot)\} \subset C\left([0, t], \mathbb{R}^{N}\right)$. Notice that $\mathcal{K} \subset \mathcal{K}_{j}^{[0, t]}$ for the previously identified $j \in \mathbb{N}$ and thus one has

$$
\begin{aligned}
& \left|\int_{0}^{t}\left[f_{n}\left(s, z_{n}(s)\right)-f(s, x(s))\right] d s\right| \\
& \quad \leq\left|\int_{0}^{t}\left[f_{n}\left(s, z_{n}(s)\right)-f\left(s, z_{n}(s)\right)\right] d s\right|+\left|\int_{0}^{t}\left[f\left(s, z_{n}(s)\right)-f(s, x(s))\right] d s\right| \\
& \quad \leq \sup _{y(\cdot) \in \mathcal{K}_{j}^{[0, t]}}\left|\int_{0}^{t}\left[f_{n}(s, y(s))-f(s, y(s))\right] d s\right|+\left|\int_{0}^{t}\left[f\left(s, z_{n}(s)\right)-f(s, x(s))\right] d s\right| .
\end{aligned}
$$


Now, in the last step of the previous chain of inequalities, the first integral goes to zero as $n \rightarrow \infty$ because, by assumption, $\left(f_{n}\right)_{n \in \mathbb{N}}$ converges to $f$ in $\sigma_{\Theta}$, and the second integral goes to zero as $n \rightarrow \infty$ because $f \in \mathfrak{W} \Theta \mathfrak{C}$ and $\left(z_{n}(\cdot)\right)_{n \in \mathbb{N}}$ converges uniformly to $x(\cdot)$ in $\left[0, b_{1}\right]$ (see $(\mathbf{W})$ in Definition 2.4). Hence, from (3.48), (3.49) and recalling that $\left(x_{0, n}\right)_{n \in \mathbb{N}}$ converges to $x_{0}$ as $n \rightarrow \infty$ one has

$$
x(t)=x_{0}+\int_{0}^{t} f(s, x(s)) d s \quad \text { for } t \in\left[0, b_{1}\right] \cap \mathbb{Q} .
$$

As a matter of fact, the equality holds on the whole interval $\left[0, b_{1}\right]$. Indeed, for any $t \in\left[0, b_{1}\right]$, if $\left(q_{n}\right)_{n \in \mathbb{N}}$ is a sequence in $\mathbb{Q}$ such that $q_{n} \uparrow t$ as $n \rightarrow \infty$, then, using (3.50) one has

$$
\begin{aligned}
\left|x(t)-x_{0}-\int_{0}^{t} f(s, x(s)) d s\right| & \leq\left|x(t)-x\left(q_{n}\right)\right|+\left|\int_{q_{n}}^{t} f(s, x(s)) d s\right| \\
& \leq\left|x(t)-x\left(q_{n}\right)\right|+\int_{q_{n}}^{t} m_{f}^{j}(s) d s
\end{aligned}
$$

where $m_{f}^{j}(\cdot) \in L_{l o c}^{1}$ is an $m$-bound for $f$ on $B_{j}$. Thus, one obtains (3.50) for all $t \in\left[0, b_{1}\right]$ due to the continuity of $x(\cdot)$ and of the Lebesgue integral.

Therefore, recalling that $z_{n}(\cdot)=x_{n}(\cdot)$ in $\left[0, b_{1}\right]$, we have that $\left(x_{n}(\cdot)\right)_{n \in \mathbb{N}}$ converges uniformly to $x(\cdot)$ in $\left[0, b_{1}\right]$ (which finishes part (ii)), and also that $x(\cdot)$ is an absolutely continuous function solving the Carathéodory initial value problem $\dot{x}=f(t, x), x(0)=x_{0}$, with $f \in \mathfrak{W} \Theta \mathfrak{C}$ (which finishes part (iii)). Notice that such a problem and its integral solution are well-defined thanks to Proposition 2.6.

Remark 3.21. A concrete example of a differential equation in $\mathfrak{W} \Theta \mathfrak{C}$ for which we can provide a solution through the application of Theorem 3.20 is given by

$$
\dot{x}=G(t, x), \quad \text { with } F_{4 n} \stackrel{n \rightarrow \infty}{\longrightarrow} G \text { in }\left(\mathfrak{W} \Theta \mathfrak{C}, \sigma_{\Theta}\right) \text {, and } F \subset \mathfrak{L} \mathfrak{C},
$$

where $F$ and $G$ are the functions defined in Example 3.15 and $\Theta=\{2 t\}$. Notice that in Example 3.15, we proved that $\left(F_{4 n}\right)_{n \in \mathbb{N}}$ converges to $G$ in $\left(\Theta \mathfrak{C}, \mathcal{T}_{\Theta}\right)$ and thus it also converges in $\left(\mathfrak{W} \Theta \mathfrak{C}, \sigma_{\Theta}\right)$.

\subsubsection{Digitization of a non-autonomous control system}

As follows, we show how some of the results contained in this thesis can be applied to study the digitization of non-autonomous control system like

$$
\dot{x}=f(t, x, u),
$$

where $f: \mathcal{U} \subset \mathbb{R} \times \mathbb{R}^{N} \times \mathbb{R}^{M} \rightarrow \mathbb{R}^{N}$ is continuous and sufficiently regular. Particularly, any digitization method of a control system consists of a systematic way of constructing differential problems of the type

$$
\dot{x}=f^{\delta}(t, x, u), \quad \text { with } t \in \mathbb{R}, x \in \mathbb{R}^{N}, u \in \mathbb{R}^{M},
$$


where $f^{\delta}$ is a Borel function which is piece-wise constant with respect to the variable $t$, and approximates $f$ in some sense that will be made clear later.

The interest of the digitization of a non-autonomous control system resides in its applications in real science. For example, digitization is useful for the investigation of numerically approximated control systems or in those cases in which one has to "sample" a vector field in a systematic way during an experiment. The importance of proving the robustness of some stability properties under a digitization method, is thus obvious. Further applications in problems of engineering or control theory can be found in L. Grüne [27] and D. Nešić et al. [40].

Let $f: \mathbb{R} \times \mathbb{R}^{2 N} \rightarrow \mathbb{R}^{N}$ be such that, for any $j \in \mathbb{N}$,

- $f$ is bounded and uniformly continuous in $\mathbb{R} \times B_{j}$ and

- there exist constants $m^{j}>0$ and $l^{j}>0$ such that

$$
|f(t, x, u)| \leq m^{j}, \quad \text { and } \quad|f(t, x, u)-f(t, y, v)| \leq l^{j}|(x, u)-(y, v)|,
$$

for all $t \in \mathbb{R}$ and $(x, u),(y, v) \in B_{j} \subset \mathbb{R}^{N+M}$.

We will consider the following digitization method: let $\delta$ be a positive number, for every $k \in \mathbb{Z}$ denote by $I_{k}$ the interval $[k \delta,(k+1) \delta]$, and define the function $f^{\delta}: \mathcal{U} \subset \mathbb{R} \times \mathbb{R}^{N} \times \mathbb{R}^{M} \rightarrow \mathbb{R}^{N}$, by

$$
f^{\delta}(t, x, u)=\frac{1}{\delta} \sum_{k \in \mathbb{Z}} \chi_{I_{k}}(t) \int_{k \delta}^{(k+1) \delta} f(s, x, u) d s
$$

where $\chi_{I}(\cdot)$ is the characteristic function of the interval $I \subset \mathbb{R}$. The function $f^{\delta}$ is piece-wise continuous with respect to $t$. Additionally, for every $\varepsilon>0$ there exists a $\delta>0$ such that for all $s, t \in \mathbb{R}$ and $\left(x_{1}, u_{1}\right),\left(x_{2}, u_{2}\right) \in B_{j}$,

$$
|t-s|+\left|\left(x_{1}-x_{2}, u_{1}-u_{2}\right)\right|<2 \delta \Rightarrow\left|f\left(t, x_{1}, u_{1}\right)-f\left(s, x_{2}, u_{2}\right)\right|<\varepsilon,
$$

because for any $j \in \mathbb{N}$ we have that $f$ is uniformly continuous in $\mathbb{R} \times B_{j}$ by assumption. Therefore, taken $\left(x_{1}, u_{1}\right),\left(x_{2}, u_{2}\right) \in B_{j}$ with $\left|\left(x_{1}-x_{2}, u_{1}-u_{2}\right)\right|<\delta$ one has

$\left|f\left(t, x_{1}, u_{1}\right)-f^{\delta}\left(t, x_{2}, u_{2}\right)\right| \leq \frac{1}{\delta} \sum_{k \in \mathbb{Z}} \chi_{I_{k}}(t) \int_{k \delta}^{(k+1) \delta}\left|f\left(t, x_{1}, u_{1}\right)-f\left(s, x_{2}, u_{2}\right)\right| d s<\varepsilon$.

Thus, one has that for any $j \in \mathbb{N}$ and $\varepsilon>0$, there exists $\delta=\delta(j, \varepsilon)>0$ such that

$$
\left\|f-f^{\delta}\right\|_{L^{\infty}\left(\mathbb{R} \times B_{j}\right)}<\varepsilon
$$

We claim that for any $\delta>0, f^{\delta} \in \mathfrak{L C}^{p}$. Firstly, notice that $f^{\delta}$ is Borel. Moreover, one has that for all $t \in \mathbb{R}$ and $(x, u) \in B_{j} \subset \mathbb{R}^{N+M}$

$$
\left|f^{\delta}(t, x, u)\right| \leq \frac{1}{\delta} \sum_{k \in \mathbb{Z}} \chi_{I_{k}}(t) \int_{I_{k}}|f(s, x, u)| d s \leq m^{j} \sum_{k \in \mathbb{Z}} \chi_{I_{k}}(t) \delta \frac{1}{\delta}=m^{j}
$$


and, for all $t \in \mathbb{R}$ and $(x, u),(y, v) \in B_{j} \subset \mathbb{R}^{N+M}$,

$$
\begin{aligned}
\left|f^{\delta}(t, x, u)-f^{\delta}(t, y, v)\right| & \leq \frac{1}{\delta} \sum_{k \in \mathbb{Z}} \chi_{I_{k}}(t) \int_{I_{k}}|f(s, x, u)-f(s, y, v)| d s \\
& \leq l^{j}|(x, u)-(y, v)| \sum_{k \in \mathbb{Z}} \chi_{I_{k}}(t) \delta \frac{1}{\delta} \\
& =l^{j}|(x, u)-(y, v)| .
\end{aligned}
$$

As a consequence, $f^{\delta}$ has the same $m$-bound and $l$-bound of $f$ on $B_{j}$ (due to the assumptions on $f$ and the chosen digitization method), and thus $f^{\delta} \in \mathfrak{L} \mathfrak{C}_{p}$.

Now, let us consider $E \subset \mathfrak{L C}_{p}\left(\mathbb{R}^{N}\right)$ composed of the functions which, for each $j \in \mathbb{N}$, admit $m^{j}$ and $l^{j}$ as an $m$-bound and an $l$-bound, respectively, on $B_{j} \subset \mathbb{R}^{N} \times \mathbb{R}^{M}$. Notice that, trivially, $E$ has $L_{l o c}^{1}$-bounded $m$-bounds and $L_{l o c}^{1}$ bounded $l$-bounds. Consequently, reasoning as in the proof of Theorem 2.26, we obtain that $E$ is closed and, thanks to Theorem 2.31, all the considered strong topologies coincide on $E$.

Furthermore, for any $g \in E$ one has that also $g_{t} \in E$ for all $t \in \mathbb{R}$, and that due to (3.51), (3.54) and (3.55), $f, f^{\delta} \in E$ for all $\delta>0$. Therefore, if $\mathcal{T}$ is any of the considered strong topologies, one has

$$
\operatorname{Hull}_{(\mathfrak{L C}, \mathcal{T})}(f) \subset E, \quad \text { and } \quad \operatorname{Hull}_{(\mathfrak{L C}, \mathcal{T})}\left(f^{\delta}\right) \subset E .
$$

In fact, (3.56) is true also for the metric topology $\mathcal{T}_{U P}$ on $\mathfrak{L} \mathfrak{C}$ used in [25]. $\mathcal{T}_{U P}$ is induced by the following family of seminorms

$$
p_{r, j}(g)=\sup _{(x, u) \in B_{j}}\left[\int_{-r}^{r}|g(t, x, u)|^{p} d t\right]^{1 / p}, \quad \text { with } r, j \in \mathbb{N} \text { and } g \in \mathfrak{L} \mathfrak{C},
$$

and define the metric

$$
d\left(g_{1}, g_{2}\right)=\sum_{j \in \mathbb{N}} \frac{1}{2^{j}}\left(\sum_{r \in \mathbb{N}} \frac{1}{2^{r}} \frac{p_{r, j}\left(g_{1}-g_{2}\right)}{1+p_{r, j}\left(g_{1}-g_{2}\right)}\right) .
$$

Notice that $\mathcal{T}_{U P}$ can be seen as a topology $\mathcal{T}_{\Theta}$ with the trivial set of moduli of continuity $\Theta_{0}=\{0\}$. In particular, for any dense and countable set $D \subset \mathbb{R}^{N+M}$ and for any suitable set of moduli of continuity $\Theta$, one has that

$$
\mathcal{T}_{D} \leq \mathcal{T}_{U P} \leq \mathcal{T}_{\Theta} \leq \mathcal{T}_{B}
$$

and thus, thanks to the $L_{l o c}^{1}$-boundedness of the $l$-bounds of $E, \mathcal{T}_{U P}$ coincides with the other topologies on $E$.

Actually, we can say a little bit more about the hull of $f$. Let us consider $\mathrm{Hull}(f)$ with respect to the compact-open topology. Since $f$ is bounded and uniformly continuous on $\mathbb{R} \times K$, where $K$ is any compact subset of $\mathbb{R}^{N+M}$, then 
Hull $(f)$ is compact (see Example 1.12 and specifically Theorem 1.13), and thus it coincides with $\operatorname{Hull}_{\left(\mathfrak{L C}_{p}, \mathcal{T}\right)}(f)$, where $\mathcal{T}$ is any of the considered strong topologies. In other words we have

$$
\operatorname{Hull}(f)=\operatorname{Hull}_{(\mathfrak{L C}, \mathcal{T})}(f) \subset E, \quad \text { where } \mathcal{T} \in\left\{\mathcal{T}_{D}, \mathcal{T}_{U P}, \mathcal{T}_{\Theta}, \mathcal{T}_{B}\right\}
$$

Furthermore, because of the previous reasonings we have that

$$
\forall h \in \operatorname{Hull}(f): h^{\delta} \in E \text {, for any } \delta>0,
$$

and thus also

$$
\operatorname{Hull}(h) \subset \operatorname{Hull}(f) \subset E, \quad \text { and } \quad \operatorname{Hull}_{(\mathfrak{L C}, \mathcal{T})}\left(h^{\delta}\right) \subset E .
$$

We recall the following theorem from [25], that can now be proved as an easy consequence of Theorem 2.35.

Theorem 3.22. Let $f: \mathbb{R} \times \mathbb{R}^{N} \rightarrow \mathbb{R}^{N}$ be bounded and uniformly continuous on $\mathbb{R} \times B_{j}$ for each $j \in \mathbb{N}$ and such that $f$ is uniformly Lipschitz continuous on each $B_{j}$, with $j \in \mathbb{N}$, that is, it satisfies (3.51). If for any $h \in \operatorname{Hull}(f)$ and any $\delta>0$ we denote by $h^{\delta}$ the digitization of $h$ through the method in (3.52), then the set

$$
G_{\delta_{0}}=\bigcup_{\substack{h \in \operatorname{Hull}(f), 0<\delta<\delta_{0}}} \operatorname{Hull}_{(\mathfrak{L C}, \mathcal{T})}\left(h^{\delta}\right),
$$

is relatively compact in $\left(\mathfrak{L} \mathfrak{C}\left(\mathbb{R}^{N}\right), \mathcal{T}\right)$, where $\mathcal{T}$ is any of the considered strong topologies. Furthermore,

$$
\lim _{\delta \rightarrow 0} \operatorname{dist}\left(\operatorname{cls}_{(\mathfrak{L C}, \mathcal{T})}\left(G_{\delta}\right), \operatorname{Hull}(f)\right)=0
$$

where $\operatorname{dist}(A, B)$ is the Hausdorff distance between two nonempty sets $A, B$ in $\left(\mathfrak{L} \mathfrak{C}\left(\mathbb{R}^{N}\right), d\right)$ (with $d(\cdot, \cdot)$ defined as in $(3.57)$ ) i.e.

$$
\operatorname{dist}(A, B):=\max \{d(A, B), d(B, A)\} \quad \text { and } \quad d(A, B):=\sup _{a \in A} \inf _{b \in B} d(a, b) .
$$

In [25], such result is preliminary to prove the persistence under digitization of the properties of local null controllability and local feedback controllability of a non-autonomous control system. Clearly, such robustness is also based on the continuous variation of the solutions with respect to the the vector fields, and in particular Theorem 3.8 can be applied.

\subsubsection{Carathéodory compartmental systems}

A compartmental system is a mathematical modelization of a real phenomenon characterized by conservation laws (e.g. mass, energy, fluid) in which there are 


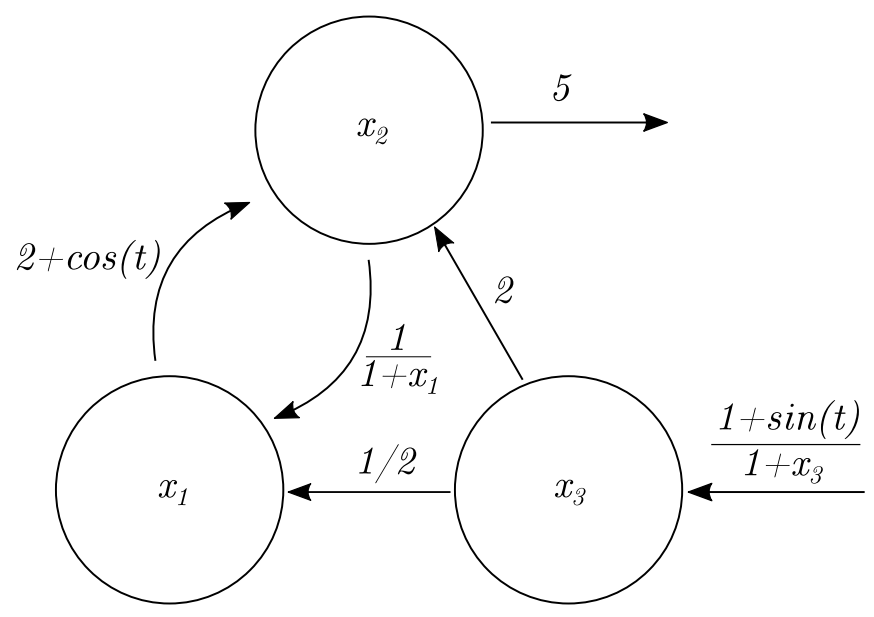

Figure 3.3: Example of a compartmental model

particles entering into a network of compartments (also called pools), travelling through them and evetually leaving the system.

The compartments are assumed to be kinetically homogeneous, that is, the material entering any compartment is instantaneously mixed with the material which is already inside that compartment. Moreover, they can represent actual containers, as for example in pharmacokinetics where they are identified with the organs of the body, or abstract sets of elements sharing the same properties, as for example in epidemiology where susceptible, infected and recovered people are considered as different compartments.

Further applications include models in biology, chemistry, demography, ecology, economic systems, epidemiology, hydrology, large-scale systems, pharmacokinetics, queuing systems, social dynamics, stochastic systems (whose state variables represent probabilities), structural vibration systems, telecommunications systems, transportation systems etc (see Haddad et al [28], Jacquez [32], Jacquez and Simon [33] and the references therein).

As follows, one finds a non-autonomous non-linear modelization for a compartmental system. Consider $B: I \times\left(\mathbb{R}^{+}\right)^{N} \rightarrow \mathbb{R}^{N \times N}$ and $s: I \times\left(\mathbb{R}^{+}\right)^{N} \rightarrow\left(\mathbb{R}^{+}\right)^{N}$, and the system

$$
\dot{x}=B(t, x) x+s(t, x)
$$

such that for all $t \in I, x \in\left(\mathbb{R}^{+}\right)^{N}$ one has

1) $b_{i i}(t, x)<0 \quad \forall i=1, \ldots, N$;

2) $b_{i j}(t, x) \geq 0 \quad \forall i \neq j$

3) $\sum_{i} b_{i j}(t, x) \leq 0 \quad \forall j=1, \ldots, N$.

The entries of the matrix-valued function $B$ represent the rate of particles moving from one compartment to the other. In this formulation such rates depends on the time $t$ and on the vector of the amount of particles in each of the 
compartment of the system, namely $x$. For every $i \in\{1, \ldots, N\}$, the entry $b_{i i}$ represents the rate of particles leaving box $i$. Assumption 1) implies that there is no creation of matter inside the compartments. For any $i \neq j$ the entry $b_{i j}(t, x)$ represents the rate of particles going from box $j$ to box $i$. As a consequence, for any $i \neq j$ the entry $b_{i j}(t, x)$ is required to be positive (see assumption 2). For every $j \in\{1, \ldots, N\}$, the sum over the entries of the column $j$ gives the rate of particles that leave the system from box $j$. Therefore, assumption 3) implies that there is no accumulation of matter inside the compartments. Finally, for any $i \in\{1, \ldots, N\}, s_{i}(t, x)$ represents the rate of particles entering the system from the external environment directly into box $i$. As an example, consider the simple compartmental system depicted in Picture 3.3. Then, we would write the compartmental matrix and the input vector using the mentioned rules, as follows,

$B(t, x)=\left[\begin{array}{ccc}-2-\cos (t) & \frac{1}{1+x_{1}} & \frac{1}{2} \\ 2+\cos (t) & -5-\frac{1}{1+x_{1}} & 2 \\ 0 & 0 & -2-\frac{1}{2}\end{array}\right] \quad$ and $\quad s(t, x)=\left[\begin{array}{c}0 \\ 0 \\ \frac{1+\sin (t)}{1+x_{3}}\end{array}\right]$.

More in general, $B(\cdot, \cdot, \cdot)$ and $s(\cdot, \cdot)$ can be Lipschitz Carathéodory functions. Such assumption allows to take into account those real phenomena in which the rate of flow or the input flow may possibly change discontinuously in time. For example, in (3.59), one can change the third component in the input vector $s(t, x)$ by

$$
s_{3}(t, x)=\frac{1+\operatorname{sgn}(\sin (t))}{1+x_{3}}
$$

where $\operatorname{sgn}(\cdot)$ denotes the sign function.

Key notions for the modelization of compartmental systems are the mean age of the system, i.e. the mean over the age of the particles inside the system at any given time, and the transit time, i.e. the mean age of mass leaving the system at any instant of time. The following formula has been suggested in [50] for the mean age of each compartment in a non-autonomous compartmental system

$$
\bar{a}_{i}(t)=\frac{\int_{0}^{+\infty} a p_{i}(a, t) d a}{\int_{0}^{+\infty} p_{i}(a, t) d a} \quad \text { for all } i \in\{1, \ldots, d\},
$$

where $p_{i}(a, t)$ is the density function of age $a$ for the mass in pool $i$ at time $t$. Following the same reasonings used in [50], one can use McKendrick-Von Förster PDE

$$
\frac{\partial p_{i}}{\partial t}+\frac{\partial p_{i}}{\partial a}=\sum_{j=1}^{d} b_{i j}(t, x) p_{j}
$$

with boundary condition $p_{i}(0, t)=s_{i}(t)$ to obtain the following ordinary differential equation describing the evolution of the mean ages of any pool.

$$
\dot{\bar{a}}_{i}(t)=1+\frac{\sum_{j=1}^{d}\left[b_{i j}(t, x) x_{j}(t)\left(\bar{a}_{j}(t)-\bar{a}_{i}(t)\right)-\bar{a}_{i}(t) s_{i}(t, x)\right]}{x_{i}(t)} .
$$


Consequently, given a solution $x(t)$ of (3.58), one may write the linear problem

$$
\dot{\bar{a}}=A(t, x(t)) \bar{a}+(1, \ldots, 1)^{T}
$$

where $A(t, x(t))$ is given by

$$
X(t)^{-1}\left[\begin{array}{cccc}
-s_{1}(t, x)-\sum_{j \neq 1} b_{1 j}(t, x) x_{j}(t) & b_{12}(t, x) x_{2}(t) & & b_{1 d}(t, x) x_{d}(t) \\
b_{21}(t, x) x_{1}(t) & -s_{2}(t, x)-\sum_{j \neq 1} b_{2 j}(t, x) x_{j}(t) & & b_{2 d}(t, x) x_{d}(t) \\
\vdots & \vdots & \ddots & \vdots \\
b_{d 1}(t, x) x_{1}(t) & b_{d 2}(t, x) x_{2}(t) & -s_{d}(t, x)-\sum_{j \neq d} b_{d j}(t, x) x_{j}(t)
\end{array}\right]
$$

with $X(t)=\operatorname{diag}\left(x_{1}(t), \ldots, x_{d}(t)\right)$ for all $t \in I$. As a consequence one obtains the system of skew product type made of the following Carathéodory ordinary differential equations

$$
\left\{\begin{array}{l}
\dot{x}=B(t, x) x+s(t, x) \\
\dot{\bar{a}}=A(t, x(t)) \bar{a}+(1, \ldots, 1)^{T} .
\end{array}\right.
$$

A continuous skew-product flow can be obtained for the triangular system (3.60) thanks to any of the Theorems $3.5,3.7$ or 3.9 , whenever $B(\cdot, \cdot)$, and $s(\cdot, \cdot)$ satisfy the relative assumptions. 


\section{Chapter 4}

\section{Pullback and global attractors for Carathéodory ODEs}

This chapter deals with pullback and global attractors for Carathéodory ODEs whose solutions are assumed to be defined up to $+\infty$. If the notion of forward attraction is transversely known in dynamical systems for the study of the long-time qualitative behavior of the solutions, the pullback attraction is instead specific of non-autonomous dynamics. In some sense, since the behavior of the trajectories of an autonomous dynamical system only depends on the elapsed time $t-t_{0}$, looking at what happens as $t \rightarrow \infty$ for a fixed $t_{0} \in \mathbb{R}$, is the same as pulling $t_{0} \rightarrow-\infty$ for a fixed $t \in \mathbb{R}$. On the contrary, for a non-autonomous dynamical system these two approaches give back different type of attractions. Specifically, we refer to pullback attraction whenever we fix a time $t \in \mathbb{R}$ and pull the initial data $x_{0}$ back to $-\infty$ to see the behavior of the corresponding trajectory at time $t$.

It is known that pullback and forward attraction are not related. In particular, a non-autonomous dynamical system can have a forward attractor without having any pullback attractor and vice versa. Moreover, such notions can be scaled up to the whole skew-product semiflow induced for such a kind of differential equation. We recommend Caraballo and Han [13] and Carvalho et al. [16] for a detailed dissertation.

The chapter is structured as follows. In Section 4.1, we include all the initial definition and results which are useful in the rest of the chapter.

In Section 4.2, we show how a continuous skew-product flow can be used to infer the existence of an attractor for a set of limit systems. In particular, starting from specific properties on the solutions of an initial problem $\dot{x}=f(t, x)$, we show how it is possible to obtain the existence of a bounded pullback attractor for the processes induced by systems with vector field in either the alpha limit set of $f$, the omega limit set of $f$, or the whole hull of $f$. Additionally, we conclude the section with a result of existence of pullback and global attractors for the whole skew-product flow. 
In Section 4.3, we provide sufficient conditions to apply the results of the previous section. In fact, several types of attractors, both for the induced process and the induced skew-product flow, are obtained. Before that, some known comparison results for Carathéodory ODEs are recalled. In Subsection 4.3.1 the size of the solutions of a Carathéodory differential system $\dot{x}=f(t, x)$ is compared with the size of the solutions of a scalar linear equation, while in Subsection 4.3.2 a comparison with a system of linear Carathéodory equations is given.

\subsection{Preliminary definitions and results}

The different types of ultimately bounded character of the solutions are subsequently defined in terms of the process associated to the corresponding dynamical system. Recall that a process can be induced by a non-autonomous differential equation if one assumes that for any $r \in \mathbb{R}$ and any $x_{0} \in \mathbb{R}^{N}$, the initial value problem $\dot{x}=f(t, x), x(r)=x_{0}$ has a unique solution $x\left(\cdot, f, r, x_{0}\right)$ defined on $[r, \infty)$. In fact, one can pose

$$
S_{f}(t+r, r) x_{0}=x\left(t+r, f, r, x_{0}\right)=x\left(t, f_{r}, x_{0}\right),
$$

where $t \geq 0$ and $r \in \mathbb{R}$, and easily check that the properties in Definition 1.5 are satisfied. In this chapter, anytime we consider a process induced by a differential equation, we implicitly assume that the relative solutions are defined up to $+\infty$.

Definition 4.1 (Uniformly ultimately bounded solutions). Consider $f \in \mathfrak{L} \mathfrak{C}$. The solutions of $\dot{x}=f(t, x)$ are said to be

- uniformly ultimately bounded if there is a positive constant $c>0$ such that for every $d>0$ there is a time $T(d)>0$ satisfying

$$
\left|S_{f}(t+r, r) x_{0}\right| \leq c \quad \text { for every } r \in \mathbb{R}, t \geq T(d) \text { and }\left|x_{0}\right| \leq d ;
$$

- uniformly ultimately bounded on $[\tau, \infty)$ if there is a positive constant $c(\tau)$ such that for every $d>0$ there is a time $T(\tau, d)>0$ such that

$$
\left|S_{f}(t+r, r) x_{0}\right| \leq c(\tau)
$$

whenever $r \geq \tau, t \geq T(\tau, d)$ and $\left|x_{0}\right| \leq d$.

The definition of pullback attractor for a process is also hereby recalled.

Definition 4.2 (Pullback attractor for a process). Consider a process $S(\cdot, \cdot)$ (see Definition 1.5) defined on $\mathbb{R}^{N}$. A family of subsets $\mathcal{A}(\cdot)=\{\mathcal{A}(t) \mid t \in \mathbb{R}\}$ of $\mathbb{R}^{N}$ is said to be a pullback attractor for the process $S(\cdot, \cdot)$ if

(i) $\mathcal{A}(t)$ is compact for each $t \in \mathbb{R}$; 
(ii) $\mathcal{A}(\cdot)$ is invariant, that is, $S(t, s) \mathcal{A}(s)=\mathcal{A}(t)$ for all $t \geq s$;

(iii) for each $t \in \mathbb{R}, \mathcal{A}(t)$ pullback attracts bounded sets at time t, i.e. for any bounded set $B \subset \mathbb{R}^{N}$ one has

$$
\lim _{s \rightarrow-\infty} \operatorname{dist}(S(t, s) B, \mathcal{A}(t))=0
$$

where $\operatorname{dist}(A, B)$ is the Hausdorff semi-distance between two nonempty sets $A, B \subset \mathbb{R}^{N}$ i.e. $\operatorname{dist}(A, B):=\sup _{x \in A} \inf _{y \in B} d(x, y)$.

(iv) $\mathcal{A}$ is the minimal family of closed sets with property (iii).

The pullback attractor is said to be bounded in the past (resp. bounded) if for all $\tau>0$ one has that $\bigcup_{t \leq \tau} \mathcal{A}(t)$ (resp. $\bigcup_{t \in \mathbb{R}} \mathcal{A}(t)$ ) is bounded.

The following characterizations of the bounded and bounded in the past pullback attractors for $S_{f}(\cdot, \cdot)$ are given in Theorem 1.17 and Corollary 1.18 of Carvalho et al. [16].

Proposition 4.3. Let $\mathcal{A}(\cdot)$ be a pullback attractor for the process $S_{f}(\cdot, \cdot)$ induced by $\dot{x}=f(t, x)$ with solutions denoted by $x(\cdot)$. Then

(i) $\mathcal{A}(t)=\{x(t) \mid x(\cdot)$ is a backward bounded solution $\}$ if $\mathcal{A}(\cdot)$ is bounded in the past, and

(ii) $\mathcal{A}(t)=\{x(t) \mid x(\cdot)$ is a global bounded solution $\}$ if $\mathcal{A}(\cdot)$ is bounded.

The notion of pullback absorbing family will also be necessary.

Definition 4.4 (Pullback absorbing sets). Let $S(\cdot, \cdot)$ be a process (see Definition 1.5) defined on $\mathbb{R}^{N}$. A family of nonempty bounded sets $\left\{B(t) \subset \mathbb{R}^{N} \mid t \in \mathbb{R}\right\}$ pullback absorbs bounded sets, if for every $t \in \mathbb{R}$ and every bounded subset $D$ of $\mathbb{R}^{N}$ there exists a time $T(t, D)>0$ such that

$$
S(t, t-s) D \subset B(t) \quad \text { for every } s \geq T(t, D) \text {. }
$$

We also say that $\left\{B(t) \subset \mathbb{R}^{N} \mid t \in \mathbb{R}\right\}$ is a pullback bounded absorbing family. If for all $t \in \mathbb{R}$ one has $B(t) \equiv B \subset \mathbb{R}^{N}$ we will say that $B$ is a pullback absorbing set.

Definition 4.5 (Pullback strongly bounded dissipative process). A process $S(\cdot, \cdot)$ (see Definition 1.5) is pullback strongly bounded dissipative on $(-\infty, \tau]$ if there exists a family $\left\{B(t) \subset \mathbb{R}^{N} \mid t \in \mathbb{R}\right\}$ of pullback bounded absorbing sets such that for every bounded subset $D \subset \mathbb{R}^{N}$, there is a time $T(\tau, D)>0$ so that

$$
S(t, t-s) D \subset B(\tau) \quad \text { for every } t \leq \tau \text { and } s \geq T(\tau, D) \text {. }
$$


Remark 4.6. In the finite dimensional case, the existence of a pullback bounded absorbing family ensures the existence of a pullback attractor (see, e.g., [16] and [36]). If in addition the family satisfies (4.3), then the pullback attractor $\{\mathcal{A}(t) \mid t \in \mathbb{R}\}$ is bounded in the past. When there is a bounded set $B$ such that for every bounded subset $D \subset \mathbb{R}^{N}$ there is a time $T(D)>0$ so that

$$
S(t, t-s) D \subset B \quad \text { for every } t \in \mathbb{R} \text { and } s \geq T(D),
$$

then there is a bounded pullback attractor.

Remark 4.7. Notice that condition (4.4) is equivalent to the uniformly ultimately bounded character of the solutions of the system.

We also recall the definitions of pullback and global attractor for a skewproduct semiflow. Consider the space of Lipschitz Carathéodory functions mapping $\mathbb{R} \times \mathbb{R}^{N}$ onto $\mathbb{R}^{N}$, endowed with a topology $\mathcal{T}$, namely $(\mathfrak{L} \mathfrak{C}, \mathcal{T})$, and $f \in \mathfrak{L} \mathfrak{C}$, so that the induced local skew-product flow

$$
\begin{array}{ccc}
\Pi: \mathcal{U} \subset \mathbb{R} \times \operatorname{Hull}_{(\mathfrak{L C}, \mathcal{T})}(f) \times \mathbb{R}^{N} & \rightarrow & \operatorname{Hull}_{(\mathfrak{L} \mathfrak{C}, \mathcal{T})}(f) \times \mathbb{R}^{N} \\
\left(t, g, x_{0}\right) & \mapsto & \left(g_{t}, x\left(t, g, x_{0}\right)\right),
\end{array}
$$

is continuous. Recall that the properties on $f$ and the topologies of continuity for (4.5) are treated in Section 3.1. In particular, we recall the following two cases:

- $f \in\left(\mathfrak{L} \mathfrak{C}, \sigma_{\Theta}\right)$ with $L_{l o c}^{1}$-equicontinuous $m$-bounds; see Theorem 3.5(i).

- $f \in\left(\mathfrak{L} \mathfrak{C}, \mathcal{T}_{D}\right)$ with $L_{l o c}^{p}$-bounded l-bounds; see Theorem 3.9(i).

Definition 4.8 (Pullback and global attractors for a skew product semiflow). Assume that for any $g \in \operatorname{Hull}_{(\mathfrak{L C}, \mathcal{T})}(f)$ and any $x_{0} \in \mathbb{R}^{N}$, the solution $x\left(\cdot, g, x_{0}\right)$ of $\dot{x}=g(t, x), x(0)=x_{0}$, is defined on $[0, \infty)$, i.e. the skew-product semiflow (4.5) is defined on $\mathbb{R}^{+} \times \operatorname{Hull}_{(\mathfrak{L C}, \mathcal{T})}(f) \times \mathbb{R}^{N}$.

- A family $\widehat{A}=\left\{A_{g} \mid g \in \operatorname{Hull}_{(\mathfrak{L C}, \mathcal{T})}(f)\right\}$ of nonempty, compact sets of $\mathbb{R}^{N}$ is said to be a pullback attractor for the skew-product semiflow if it is invariant, i.e.

$$
x\left(t, g, A_{g}\right)=A_{g_{t}} \quad \text { for each } t \geq 0 \text { and } g \in \operatorname{Hull}_{(\mathfrak{L C}, \mathcal{T})}(f),
$$

and, for every nonempty bounded set $D$ of $\mathbb{R}^{N}$ and every $g \in \operatorname{Hull}_{(\mathfrak{L C}, \mathcal{T})}(f)$ one has

$$
\lim _{t \rightarrow \infty} \operatorname{dist}\left(x\left(t, g_{-t}, D\right), A_{g}\right)=0,
$$

where $\operatorname{dist}(A, B)$ denotes the Hausdorff semi-distance of two nonempty sets $A$, $B$ of $\mathbb{R}^{N}$.

A pullback attractor for the skew-product flow is said to be bounded if

$$
\bigcup_{g \in \operatorname{Hull}_{(\mathfrak{L C}, \mathcal{T})}(f)} A_{g} \text { is bounded. }
$$


- A compact set $\mathcal{A}$ of $\operatorname{Hull}_{(\mathfrak{L C}, \mathcal{T})}(f) \times \mathbb{R}^{N}$ is said to be a global attractor for the skew-product semiflow if it is the maximal nonempty compact subset of $\operatorname{Hull}_{(\mathfrak{L C}, \mathcal{T})}(f) \times \mathbb{R}^{N}$ which is $\Pi$-invariant, i.e.

$$
\Pi(t, \mathcal{A})=\mathcal{A} \quad \text { for each } t \geq 0
$$

and attracts all compact subsets $\mathcal{D}$ of $\operatorname{Hull}_{(\mathfrak{L C}, \mathcal{T})}(f) \times \mathbb{R}^{N}$, i.e.

$$
\lim _{t \rightarrow \infty} \operatorname{dist}(\Pi(t, \mathcal{D}), \mathcal{A})=0,
$$

where now $\operatorname{dist}(\mathcal{B}, \mathcal{C})$ denotes the Hausdorff semi-distance of two nonempty sets $\mathcal{B}, \mathcal{C}$ of $\operatorname{Hull}_{(\mathfrak{L C}, \mathcal{T})}(f) \times \mathbb{R}^{N}$.

Remark 4.9. We aim to provide a little bit of additional information about the relationship between the pullback attractor of a skew-product semiflow and the behavior of the solutions of each process composing the skew-product semiflow in Definition 4.8. Particularly, for each $g \in \operatorname{Hull}_{(\mathfrak{L} \mathfrak{C}, \mathcal{T})}(f)$, we would like to clarify who is the set $A_{g} \subset \mathbb{R}^{N}$ in the previous definition of the pullback attractor of the skew-product semiflow $\widehat{A}=\left\{A_{g} \mid g \in \operatorname{Hull}_{(\mathfrak{L C}, \mathcal{T})}(f)\right\}$.

Recalling that for every $g \in \operatorname{Hull}_{(\mathfrak{L C}, \mathcal{T})}(f)$ one has

$$
S_{g}(s+r, r) x_{0}=x\left(s, g_{r}, x_{0}\right),
$$

then, for every nonempty bounded set $D$ of $\mathbb{R}^{N}$, (4.7) becomes

$$
\lim _{t \rightarrow \infty} \operatorname{dist}\left(S_{g}(0,-t) D, A_{g}\right)=0,
$$

which implies that, for the given process $S_{g}(\cdot, \cdot), A_{g}$ pullback attracts bounded sets at time 0 . Now, for every $\tau \in \mathbb{R}$ one can write (4.9) for $g_{\tau}$ and $A_{g_{\tau}}$ instead of $g$ and $A_{g}$, respectively, i.e.

$$
\lim _{t \rightarrow \infty} \operatorname{dist}\left(S_{g_{\tau}}(0,-t) D, A_{g_{\tau}}\right)=0 .
$$

Nevertheless, using (4.8) twice, we also have

$$
S_{g_{\tau}}(0,-t) D=x\left(t, g_{\tau-t}, D\right)=S_{g}(\tau, \tau-t) D,
$$

and thus (4.10) can be written as

$$
\lim _{t \rightarrow \infty} \operatorname{dist}\left(S_{g}(\tau, \tau-t) D, A_{g_{\tau}}\right)=0,
$$

which implies that, for the given process $S_{g}(\cdot, \cdot), A_{g_{\tau}}$ pullback attracts bounded sets at time $\tau$. Therefore, as a consequence of the invariance contained in (4.6) and of the fact that $A_{g}$ is taken compact for any $g \in \operatorname{Hull}_{(\mathfrak{L C}, \mathcal{T})}(f)$, we deduce that the process $S_{g}(\cdot, \cdot)$ has a pullback attractor. In particular if

$$
\mathcal{A}_{g}=\left\{A(\tau)=A_{g_{\tau}} \mid \tau \in \mathbb{R}\right\}
$$


is the minimal family of closed sets satisfying (4.11), then $\mathcal{A}_{g}$ is the pullback attractor for the process $S_{g}(\cdot, \cdot)$. On the other hand if for any $g \in \operatorname{Hull}_{(\mathfrak{L C}, \mathcal{T})}(f)$, the induced process $S_{g}(\cdot, \cdot)$ has a pullback attractor and $A_{g}$ denotes the section at time 0 of the pullback attractor of $S_{g}(\cdot, \cdot)$, then one has that

$$
\widehat{A}=\left\{A_{g} \mid g \in \operatorname{Hull}_{(\mathfrak{L C}, \mathcal{T})}(f)\right\}
$$

is a pullback attractor for the skew product flow on $\operatorname{Hull}_{(\mathfrak{L C}, \mathcal{T})}(f)$.

\subsection{Pullback attractors and the skew-product flow}

This section deals with results of existence of pullback and global attractors for Carathéodory ODEs as a consequence of the continuity of the skew-product flow. In particular, we show how, starting from specific properties on the solutions of an initial problem $\dot{x}=f(t, x)$, it is possible to obtain the existence of a bounded pullback attractor for the processes induced by systems with vector field in either the alpha limit set of $f$, the omega limit set of $f$, or the whole hull of $f$. Furthermore, conditions for the existence of pullback and global attractors for the induced skew-product flow are also provided.

Theorem 4.10. Let $f$ be in $\mathfrak{L} \mathfrak{C}$ and $\mathcal{T}$ be a topology such that the induced local skew-product flow (4.5) is continuous, and assume that

- for any $g \in\left\{f_{s} \mid s \leq 0\right\} \cup \mathbb{A}(f)$ and $x_{0} \in \mathbb{R}^{N}$, the solution $x\left(\cdot, g, x_{0}\right)$ of $\dot{x}=g(t, x), x(0)=x_{0}$, is defined on $[0, \infty)$;

- there is a $\tau \in \mathbb{R}$ for which the process $S_{f}(\cdot, \cdot)$ is strongly pullback bounded dissipative on $(-\infty, \tau]$.

Then, for any function $g \in \mathbb{A}(f)$, the solutions of $\dot{x}=g(t, x)$ are uniformly ultimately bounded. In particular, the induced process $S_{g}(\cdot, \cdot)$ has a bounded pullback attractor.

Proof. Let $D$ be a bounded set. By hypothesis, there are $c=c(\tau)>0$ and $T(D)=T(\tau, D)>0$ such that for each $x_{0} \in D$ one has

$$
\left|S_{f}(t, t-s) x_{0}\right|=\left|x\left(s, f_{t-s}, x_{0}\right)\right| \leq c \quad \text { for } t \leq \tau \text { and } s \geq T(D) .
$$

If $g=\lim _{n \rightarrow \infty} f_{t_{n}}$ with $t_{n} \downarrow-\infty$, then we have $g_{t-s}=\lim _{n \rightarrow \infty} f_{t_{n}+t-s}$ and by the continuity of the semiflow

$$
\left.\mid S_{g}(t, t-s) x_{0}\right)|=| x\left(s, g_{t-s}, x_{0}\right)|=| \lim _{n \rightarrow \infty} x\left(s, f_{t_{n}+t-s}, x_{0}\right) \mid .
$$

Finally, there exists $n_{0} \in \mathbb{N}$ such that, if $n \geq n_{0}$, then $t_{n}+t \leq \min \{0, \tau\}$, and thus

$$
\left|S_{g}(t, t-s) x_{0}\right| \leq c \quad \text { for every } t \in \mathbb{R} \text { and } s \geq T(D) .
$$

Therefore, from Remark 4.7 the solutions of $\dot{x}=g(t, x)$ are uniformly ultimately bounded and, from Remark 4.6, a bounded pullback attractor exists. 
Analogously, we give conditions to have a bounded pullback attractor for the process induced by $\dot{x}=g(t, x)$, when $g \in \mathfrak{L} \mathfrak{C}$ is any function in the omega limit set $\mathbb{O}(f)$.

Theorem 4.11. Let $f$ be in $\mathfrak{L} \mathfrak{C}$ and $\mathcal{T}$ be a topology such that the induced local skew-product flow (4.5) is continuous, and assume that

- for any $g \in\left\{f_{s} \mid s \geq 0\right\} \cup \mathbb{O}(f)$, and $x_{0} \in \mathbb{R}^{N}$, the solution $x\left(\cdot, g, x_{0}\right)$ of $\dot{x}=g(t, x), x(0)=x_{0}$, is defined on $[0, \infty)$;

- there is a $\tau \in \mathbb{R}$ for which the solutions of $\dot{x}=f(t, x)$ are uniformly ultimately bounded on $[\tau, \infty)$.

Then, for any function $g \in \mathbb{O}(f)$, the solutions of $\dot{x}=g(t, x)$ are uniformly ultimately bounded and the induced process $S_{g}(\cdot, \cdot)$ has a bounded pullback attractor.

Proof. From (4.2) one has that

$$
\left|S_{f}(t+s, s) x_{0}\right|=\left|x\left(t+s, f, s, x_{0}\right)\right|=\left|x\left(t, f_{s}, x_{0}\right)\right| \leq c(\tau),
$$

whenever $s \geq \tau, t \geq T(\tau, d)$ and $\left|x_{0}\right| \leq d$. Since $g \in \mathbb{O}(f)$ there is a sequence $t_{n} \uparrow \infty$ with $\lim _{n \rightarrow \infty} f_{t_{n}}=g$. Thus, $g_{r}=\lim _{n \rightarrow \infty} f_{t_{n}+r}$ and by the continuity of the solutions

$$
\left|S_{g}(t+r, r) x_{0}\right|=\left|x\left(t, g_{r}, x_{0}\right)\right|=\left|\lim _{n \rightarrow \infty} x\left(t, f_{t_{n}+r}, x_{0}\right)\right| .
$$

Since there is $n_{0} \in \mathbb{N}$ such that, if $n \geq n_{0}$, then $t_{n}+r \geq \max \{0, \tau\}$, we conclude that

$$
\left|S_{g}(t+r, r) x_{0}\right| \leq c(\tau) \quad \text { whenever } r \in \mathbb{R}, t \geq T(\tau, d) \text { and }\left|x_{0}\right| \leq d,
$$

that is, the solutions of $\dot{x}=g(t, x)$ are uniformly ultimately bounded, as claimed. As for Theorem 4.10, from Remarks 4.7 and 4.6 we obtain the thesis.

Finally, we give conditions to have a bounded pullback attractor for the process induced by $\dot{x}=g(t, x)$, when $g \in \mathfrak{L} \mathfrak{C}$ is any function in the hull of $f$.

Theorem 4.12. Let $f$ be in $\mathfrak{L} \mathfrak{C}$ and $\mathcal{T}$ be a topology such that the induced local skew-product flow (4.5) is defined on $\mathbb{R}^{+} \times \operatorname{Hull}_{(\mathfrak{L C}, \mathcal{T})}(f) \times \mathbb{R}^{N}$ and it is continuous. If there is a pullback bounded absorbing set B satisfying (4.4), then, for any $g \in$ $\operatorname{Hull}_{(\mathfrak{L C}, \mathcal{T})}(f)$, one has that the solutions of $\dot{x}=g(t, x)$ are uniformly ultimately bounded and the induced process $S_{g}(\cdot, \cdot)$ has a bounded pullback attractor.

Proof. First, notice that $\operatorname{Hull}_{(\mathfrak{L C}, \mathcal{T})}(f)=\mathbb{A}(f) \cup \mathbb{O}(f) \cup\left\{f_{\tau} \mid \tau \in \mathbb{R}\right\}$. Moreover, condition (4.4) implies that the assumptions of Theorems 4.10 and 4.11 are satisfied, as shown in Remarks 4.7 and 4.6. Therefore, if $g \in \mathbb{A}(f)$ (resp. $g \in \mathbb{O}(f)$ ) the result follows from Theorem 4.10 (resp. Theorem 4.11). If $g$ is $f$, or one of its time-translations, the uniformly ultimately bounded character of the solutions cames again from Remark 4.7, which together with Remark 4.6 allows to end the proof. 
The next result provides the existence of a pullback attractor as well as a global attractor (when $\operatorname{Hull}_{(\mathfrak{L C}, \mathcal{T})}(f)$ is compact) of the skew-product semiflow (4.5) and the relation between them. We denote by $x(t, f, D)$ the subset of $\mathbb{R}^{N}$ given by

$$
x(t, f, D)=\left\{x\left(t, f, x_{0}\right) \mid x_{0} \in D\right\} .
$$

Theorem 4.13. Let $f$ be in $\mathfrak{L} \mathfrak{C}$ and $\mathcal{T}$ be a topology such that the induced skewproduct semiflow (4.5) is defined on $\mathbb{R}^{+} \times \operatorname{Hull}_{(\mathfrak{L C}, \mathcal{T})}(f) \times \mathbb{R}^{N}$ and it is continuous. Assume that there is a bounded set $B \subset \mathbb{R}^{N}$ such that for each nonempty bounded set $D$ there is a time $T(D)$ such that

$$
x\left(t, f_{s}, D\right) \subset B \quad \text { whenever } t \geq T(D)
$$

for every $s \in \mathbb{R}$. Then

(i) there exists a unique bounded pullback attractor $\widehat{A}=\left\{A_{g} \mid g \in \operatorname{Hull}_{(\mathfrak{L C}, \mathcal{T})}(f)\right\}$ of the skew-product semiflow (4.5) given by

$$
A_{g}=\bigcap_{\tau \geq 0} \overline{\bigcup_{t \geq \tau} x\left(t, g_{-t}, B\right)} \quad \text { for each } g \in \operatorname{Hull}_{(\mathfrak{L C}, \mathcal{T})}(f)
$$

(ii) if $\operatorname{Hull}_{(\mathfrak{L C}, \mathcal{T})}(f)$ is compact, there is a global attractor of the skew-product semiflow (4.5) given by

$$
\mathcal{A}=\bigcap_{\tau \geq 0} \overline{\bigcup_{t \geq \tau} \Pi\left(t, \operatorname{Hull}_{(\mathfrak{L C}, \mathcal{T})}(f) \times B\right)}=\bigcup_{g \in \operatorname{Hull}_{(\mathfrak{L C}, \mathcal{T})}(f)}\left\{\{g\} \times A_{g}\right\} .
$$

Proof. First, from the continuity of the skew-product flow, we deduce that

$$
x(t, g, D) \subset B \quad \text { for every } t \geq T(D) \text { and every } g \in \operatorname{Hull}_{(\mathfrak{L C}, \mathcal{T})}(f) .
$$

Therefore, among other references, (i) follows from Theorem 3.20 of [36]. The existence of a global attractor $\mathcal{A}$ under the compactness of the base $\operatorname{Hull}_{(\mathfrak{L C}, \mathcal{T})}(f)$ follows from Theorem 2.2 of Cheban et al. [18] and, as shown in Theorem 16.2 of [16], $A_{g}$ is the section of $\mathcal{A}$ over $g$, that is,

$$
\mathcal{A}=\bigcup_{g \in \operatorname{Hull}_{(\mathfrak{L C}, \mathcal{T})}(f)}\left\{\{g\} \times A_{g}\right\}
$$

which finishes the proof.

Remark 4.14. Notice that, because of the relation

$$
x\left(t, f_{s}, D\right)=S_{f}(t+s, s) D, \quad f \in \mathfrak{L} \mathfrak{C}, s, t \in \mathbb{R}, D \subset \mathbb{R}^{N},
$$

condition (4.12) is equivalent to (4.4), that is, the process induced by $f$ has a pullback bounded absorbing set $B$. 
Remark 4.15. Under the assumptions of Theorem 4.13(i-ii), in general the pullback attractors $\left\{A_{g_{t}} \mid t \in \mathbb{R}\right\}$ with $g \in \operatorname{Hull}_{(\mathfrak{L C}, \mathcal{T})}(f)$ have no forward attraction properties for the corresponding processes. However, the global attractor $\mathcal{A}$ always exhibits collective properties of forward attractivity (see [14]).

Finally, from Theorems 4.10, 4.11 and 4.13 we obtain the corresponding results for the induced skew-product flow on $\mathbb{A}(f) \times \mathbb{R}^{N}$ and $\mathbb{O}(f) \times \mathbb{R}^{N}$.

Corollary 4.16. Let $f \in \mathfrak{L} \mathfrak{C}$ and $\mathcal{T}$ be a topology such that the induced local skew-product flow (4.5) is continuous, and assume that

- for any $g \in\left\{f_{s} \mid s \leq 0\right\} \cup \mathbb{A}(f)$, and any $x_{0} \in \mathbb{R}^{N}$, the solution $x\left(\cdot, g, x_{0}\right)$ of $\dot{x}=g(t, x), x(0)=x_{0}$ is defined on $[0, \infty)$;

- there is a $\tau \in \mathbb{R}$ for which $S_{f}(\cdot, \cdot)$ is strongly pullback bounded dissipative on $(-\infty, \tau]$.

Then, (i) and (ii) of Theorems 4.13 hold for the skew-product flow on $\mathbb{A}(f) \times \mathbb{R}^{N}$.

Corollary 4.17. Let $f \in \mathfrak{L} \mathfrak{C}$ and $\mathcal{T}$ be a topology such that the induced local skew-product flow (4.5) is continuous, and assume that

- for any $g \in\left\{f_{s} \mid s \geq 0\right\} \cup \mathbb{O}(f)$, and any $x_{0} \in \mathbb{R}^{N}$ the solution $x\left(\cdot, g, x_{0}\right)$ of $\dot{x}=g(t, x), x(0)=x_{0}$ is defined on $[0, \infty)$;

- there is a $\tau \in \mathbb{R}$ for which the solutions of $\dot{x}=f(t, x)$ are uniformly ultimately bounded on $[\tau, \infty)$.

Then, (i) and (ii) of Theorems 4.13 hold for the skew-product flow on $\mathbb{O}(f) \times \mathbb{R}^{N}$.

\subsection{Comparison results for Carathéodory ODEs}

This section provides sufficient conditions under which the abstract results of subsection 4.2 can be applied. In fact, several types of attractors, both for the induced process and the induced skew-product flow, are obtained. Such results are based on comparison theorems for the solutions of a Carathéodory differential system which are recalled at the beginning of the section.

In Section 4.3.1, the size of the solutions of a Carathéodory differential system $\dot{x}=f(t, x)$ is compared with the size of the solutions of a scalar linear equation, while in Section 4.3.2 a comparison with a system of linear Carathéodory equations is carried out.

We firstly recall the following comparison result for the scalar case from Olech and Opial [44]. 
Proposition 4.18. Consider $f: \mathbb{R} \times \mathbb{R} \rightarrow \mathbb{R}, f \in \mathfrak{L} \mathfrak{C}, x_{0} \in \mathbb{R}$ and denote by $x\left(\cdot, f, t_{0}, x_{0}\right):\left[t_{0}, t_{1}\right] \rightarrow \mathbb{R}$ the solution of the Cauchy Problem $\dot{x}=f(t, x)$, $x\left(t_{0}\right)=x_{0}$. If $z:\left[t_{0}, t_{1}\right] \rightarrow \mathbb{R}$ is an absolutely continuous function such that

$$
z(0) \leq x_{0} \quad \text { and } \quad \dot{z}(t) \leq f(t, z(t)) \quad \text { for a.e. } t \in\left[t_{0}, t_{1}\right]
$$

then one has

$$
z(t) \leq x\left(t, f, t_{0}, x_{0}\right) \quad \text { for all } t \in\left[t_{0}, t_{1}\right] .
$$

Moreover, if, in particular, $z(0)<x_{0}$, then one has

$$
z(t)<x\left(t, f, t_{0}, x_{0}\right) \quad \text { for all } t \in\left[t_{0}, t_{1}\right] .
$$

Proof. The first part of the proof is a consequence of the comparison theorem contained in [44, Théorème 1 p.250]. If $z(0)<x_{0}$, consider $x_{1} \in \mathbb{R}$ such that $z(0)<x_{1}<x_{0}$. Then, from the first part and by the uniqueness of the solution for the Cauchy Problem $\dot{x}=f(t, x), x\left(t_{0}\right)=x_{0}$, one has that

$$
z(t) \leq x\left(t, f, t_{0}, x_{1}\right)<x\left(t, f, t_{0}, x_{0}\right), \quad \text { for all } t \in\left[t_{0}, t_{1}\right]
$$

which concludes the proof.

From the scalar case a comparison result for the vectorial case can be deduced. Let us firstly recall some notation. For every $i=1, \ldots, N$ the $i$ th component of $x \in \mathbb{R}^{N}$ will be denoted by $x_{i}$. Moreover, if we write $x \geq 0$ we mean that for all $i=1, \ldots, N$ one has $x_{i} \geq 0$, whereas we will write $x \gg 0$ if for every $i=1, \ldots N$ one has $x_{i}>0$. The space $\left(\mathbb{R}^{N}\right)^{+}$will denote the set of points $x \in \mathbb{R}^{N}$ such that $x \geq 0$. Analogously, the $i$ th component of a vector function $f: \mathbb{R} \times \mathbb{R}^{N} \rightarrow \mathbb{R}^{N}$ will be denoted by $f_{i}$.

Proposition 4.19. Consider $f \in \mathfrak{L} \mathfrak{C}$ and assume that for every $z, x \in \mathbb{R}^{N}$ with $z \leq x$ the following condition of monotonocity is satisfied:

$$
z_{i}=x_{i} \text { for some } i=1, \ldots, N \Rightarrow f_{i}(t, z) \leq f_{i}(t, x) \quad \text { for a.e. } t \in \mathbb{R} \text {. }
$$

Fixed $x_{0} \in \mathbb{R}^{N}$, and denoted by $x\left(\cdot, f, t_{0}, x_{0}\right):\left[t_{0}, t_{1}\right] \rightarrow \mathbb{R}^{N}$ the unique solution of the Cauchy Problem $\dot{x}=f(t, x), x\left(t_{0}\right)=x_{0}$. If $z:\left[t_{0}, t_{1}\right] \rightarrow \mathbb{R}^{N}$ is an absolutely continuous function such that

$$
z(0) \leq x_{0} \quad \text { and } \quad \dot{z}(t) \leq f(t, z(t)) \quad \text { for a.e. } t \in\left[t_{0}, t_{1}\right]
$$

then one has that

$$
z(t) \leq x\left(t, f, t_{0}, x_{0}\right) \quad \text { for all } t \in\left[t_{0}, t_{1}\right]
$$


Proof. The case $N=1$ is proved in Proposition 4.18. Consider $N \geq 2$ and, in order to simplify the notation, pose $x(\cdot)=x\left(\cdot, f, t_{0}, x_{0}\right)$. Firstly we prove that

$$
z(0) \ll x_{0} \Rightarrow z(t) \ll x(t) \quad \text { for all } t \in\left[t_{0}, t_{1}\right] .
$$

Assume, on the contrary, that there exists $\tau \in\left[t_{0}, t_{1}\right]$ such that the previous inequality is not true at time $\tau$ for one of the components. In other words, and assuming by simplicity that such component is the first one, we have that

$$
z(t) \ll x(t) \text { for all } t \in\left[t_{0}, \tau\right) \text { and } z_{1}(\tau)=x_{1}(\tau) \text {. }
$$

Consider the function $g: \mathbb{R} \times \mathbb{R} \rightarrow \mathbb{R}$ defined by

$$
g(t, y):=f_{1}\left(t, y, x_{2}(t), \ldots, x_{N}(t)\right)
$$

and notice that, thanks to (4.14) and (4.13), one has

$$
\dot{z}_{1}(t) \leq f_{1}(t, z(t)) \leq f_{1}\left(t, z_{1}(t), x_{2}(t), \ldots, x_{N}(t)\right)=g\left(t, z_{1}(t)\right),
$$

for almost every $t \in\left[t_{0}, \tau\right]$. Therefore, due to Proposition 4.18 we have that

$$
z_{1}(t)<y\left(t, g, t_{0},\left(x_{0}\right)_{1}\right) \quad \text { for all } t \in\left[t_{0}, \tau\right],
$$

where $y\left(\cdot, g, t_{0},\left(x_{0}\right)_{1}\right)$ denotes the solution of the scalar problem $\dot{y}=g(t, y)$ with $y\left(t_{0}\right)=\left(x_{0}\right)_{1}$. However, due to (4.18), one has that $y\left(t, g, t_{0},\left(x_{0}\right)_{1}\right)=$ $y\left(t, f_{1}, t_{0},\left(x_{0}\right)_{1}\right)$ and for the uniqueness of the solution for the Cauchy Problem $\dot{x}=f(t, x)$ with $x\left(t_{0}\right)=x_{0}$, one has

$$
y\left(\cdot, f_{1}, t_{0},\left(x_{0}\right)_{1}\right)=x_{1}\left(\cdot, f, t_{0}, x_{0}\right) .
$$

Therefore, one can write (4.19) as

$$
z_{1}(t)<x_{1}\left(t, f, t_{0}, x_{0}\right) \quad \text { for all } t \in\left[t_{0}, \tau\right] .
$$

However, such inequality evaluated at time $\tau$, contradicts (4.17). As a consequence we obtain (4.16). Now let $x_{0} \in \mathbb{R}^{N}$ be such that $z(0) \leq x_{0}$. Then, taken $\varepsilon>0$ we have that $z(0) \ll x_{0}+\varepsilon 1$, where 1 denotes the $N$-dimensional vector of ones. Then, as a consequence of the first part of the proof, one has

$$
z(t) \ll x\left(t, f, t_{0}, x_{0}+\varepsilon 1\right) \quad \text { for all } t \in\left[t_{0}, t_{1}\right],
$$

and thanks to the continuity of the solutions with respect to the variation of the initial condition (Theorem 1.3), one obtains (4.15) as $\varepsilon \rightarrow 0$, which finishes the proof.

Remark 4.20. If in Proposition 4.19 condition (4.14) is changed for

$$
x_{0} \leq z(0) \quad \text { and } \quad f(t, z(t)) \leq \dot{z}(t) \text { for a.e. } t \in\left[t_{0}, t_{1}\right],
$$

then, one obtains

$$
x\left(t, f, t_{0}, x_{0}\right) \leq z(t), \quad \text { for all } t \in\left[t_{0}, t_{1}\right] .
$$




\subsubsection{Comparison with a scalar Carathéodory linear equa- tion}

Consider a Carathéodory differential system $\dot{x}=f(t, x)$ and the condition below for $f \in \mathfrak{L} \mathfrak{C}$ :

$\left(\mathbf{H}_{1}\right)$ there exist $\alpha(\cdot), \beta(\cdot) \in L_{l o c}^{1}$, with $\beta(\cdot)$ non-negative, such that

$$
2\langle f(t, x), x\rangle \leq \alpha(t)|x|^{2}+\beta(t) \quad \text { for a.e. }(t, x) \in \mathbb{R}^{N+1},
$$

where $\langle\cdot, \cdot\rangle$ represents the scalar product in $\mathbb{R}^{N}$.

This assumption implies the following inequality for the solutions of $\dot{x}=f(t, x)$.

Proposition 4.21. Assume that $\left(\mathbf{H}_{1}\right)$ holds for $f \in \mathfrak{L} \mathfrak{C}$. If $x(\cdot)$ is a solution of $\dot{x}=f(t, x)$ defined on an interval $I$, then it satisfies

$$
2\langle f(t, x(t)), x(t)\rangle \leq \alpha(t)|x(t)|^{2}+\beta(t) \quad \text { for a.e. } t \in I .
$$

Proof. Let $V \subset \mathbb{R} \times \mathbb{R}^{N}$ be such that meas $\mathbb{R}_{\mathbb{R}^{1+N}}\left(\mathbb{R}^{1+N} \backslash V\right)=0$ and

$$
2\langle f(t, x), x\rangle \leq \alpha(t)|x|^{2}+\beta(t) \quad \text { for all }(t, x) \in V .
$$

Consider the set $E=\left\{(t, \varepsilon) \in I \times B_{1} \mid(t, x(t)+\varepsilon) \in V\right\}$, where $B_{1}$ is the closed ball of $\mathbb{R}^{N}$ centered at the origin and with radius 1 , and for any $t \in I$ denote by $E_{t}$ the section in $t$ of $E$, i.e. $E_{t}=\left\{\varepsilon \in B_{1} \mid(t, \varepsilon) \in E\right\}$. Moreover, given $t \in I$ one has that $x(t)+\left(B_{1} \backslash E_{t}\right) \subset B_{r} \backslash V_{t}$ for some $r$, and hence meas $\mathbb{R}_{\mathbb{R}^{N}}\left(B_{1} \backslash E_{t}\right)=0$ for almost every $t \in I$. Then, applying Fubini's theorem twice, one has

$$
\operatorname{meas}_{\mathbb{R}}(I) \cdot \operatorname{meas}_{\mathbb{R}^{N}}\left(B_{1}\right)=\operatorname{meas}_{\mathbb{R}^{1+N}}(E)=\int_{\mathbb{R}^{N}} \operatorname{meas}_{\mathbb{R}}\left(E_{\varepsilon}\right) d \varepsilon,
$$

where $E_{\varepsilon}$ denotes the section of $E$ for any fixed $\varepsilon \in B_{1}$. Therefore, one has $\operatorname{meas}_{\mathbb{R}}\left(E_{\varepsilon}\right)=\operatorname{meas}_{\mathbb{R}}(I)$ for almost every $\varepsilon \in B_{1}$. Now, let $\left(\varepsilon_{n}\right)_{n \in \mathbb{N}} \subset B_{1}$ be such that

$$
\varepsilon_{n} \stackrel{n \rightarrow \infty}{\longrightarrow} 0 \quad \text { and } \quad \operatorname{meas}_{\mathbb{R}}\left(E_{\varepsilon_{n}}\right)=\operatorname{meas}_{\mathbb{R}}(I) \quad \forall n \in \mathbb{N} .
$$

As a consequence, taking $J=\bigcap_{n \in \mathbb{N}} E_{\varepsilon_{n}}$ we deduce that

$$
2\left\langle f\left(t, x(t)+\varepsilon_{n}\right), x(t)+\varepsilon_{n}\right\rangle \leq \alpha(t)\left|x(t)+\varepsilon_{n}\right|^{2}+\beta(t) \quad \forall t \in J,
$$

and as $n \rightarrow \infty$ we obtain $(4.20)$ because $\operatorname{meas}_{\mathbb{R}}(I)=\operatorname{meas}_{\mathbb{R}}(J)$.

Remark 4.22. If $f \in \mathfrak{L} \mathfrak{C}$ satisfies $\left(\mathbf{H}_{1}\right)$ then, considering the Cauchy problem $\dot{x}=f(t, x), x\left(t_{0}\right)=x_{0}$, and denoting by $x(\cdot)$ its solution, from (4.20) and $|x(r)|^{2}=$ $\langle x(r), x(r)\rangle$ one has that for a.e. $r \in \mathbb{R}$

$$
\frac{d}{d r}|x(r)|^{2}=2\langle x(r), \dot{x}(r)\rangle=2\langle x(r), f(r, x(r))\rangle \leq \alpha(r)|x(r)|^{2}+\beta(r) .
$$


Therefore, if we pose $y(r)=|x(r)|^{2}$ and apply Proposition 4.18 and the variation of constants formula, we obtain that

$$
|x(t)|^{2} \leq \exp \left(\int_{t_{0}}^{t} \alpha(u) d u\right)\left|x_{0}\right|^{2}+\int_{t_{0}}^{t} \beta(r) \exp \left(\int_{r}^{t} \alpha(u) d u\right) d r
$$

for every $t>t_{0}$. As a consequence, the solutions of $\dot{x}=f(t, x)$ are defined on $\left[t_{0}, \infty\right)$ and thus a process $S_{f}(\cdot, \cdot)$ can be induced as in (4.1).

In addition to $\left(\mathbf{H}_{1}\right)$ we also consider the following conditions:

$\left(\mathbf{H}_{2}\right)$ the equation $\dot{y}=\alpha(t) y$ has exponential dichotomy on $(-\infty, 0]$ with projection $P=\mathrm{Id}$, that is, there are constants $\alpha_{1}>0$ and $K \geq 1$ such that

$$
\exp \left(\int_{s}^{t} \alpha(u) d u\right) \leq K e^{-\alpha_{1}(t-s)} \quad \text { for } s \leq t \leq 0
$$

$\left(\mathbf{H}_{3}\right)$ the set of functions $\left\{\beta_{t}(\cdot)\right\}_{t \in \mathbb{R}}$ is $L_{l o c}^{1}$-bounded,

Assumptions $\left(\mathbf{H}_{1}\right),\left(\mathbf{H}_{2}\right)$ and $\left(\mathbf{H}_{3}\right)$ allow to obtain that the process $S_{f}(\cdot, \cdot)$ is strongly pullback bounded dissipative on $(-\infty, \tau]$ for all $\tau \in \mathbb{R}$ (see Definition 4.3), as shown in the next result.

Theorem 4.23. Consider $f \in \mathfrak{L} \mathfrak{C}$ and assume that $\left(\mathbf{H}_{1}\right),\left(\mathbf{H}_{2}\right)$ and $\left(\mathbf{H}_{3}\right)$ hold. Therefore the induced process $S_{f}(\cdot, \cdot)$ is strongly pullback bounded dissipative on $(-\infty, \tau]$ for all $\tau \in \mathbb{R}$. Consequently, there exists a pullback attractor which is bounded in the past.

Proof. Consider the nondecreasing function $K(\cdot) \geq 1$ defined by

$$
K(t):= \begin{cases}K & \text { if } t \leq 0 \\ K e^{\alpha_{1} t} N(t) & \text { if } t>0\end{cases}
$$

where $N(t):=\exp \left(\int_{0}^{t}|\alpha(u)| d u\right)$. First we check that one has

$$
\exp \left(\int_{s}^{r} \alpha(u) d u\right) \leq K(t) e^{-\alpha_{1}(r-s)} \quad \text { for } s \leq r \leq t,
$$

that is, for any $t \in \mathbb{R}$, the equation $\dot{y}=\alpha(t) y$ has exponential dichotomy on $(-\infty, t]$ with projection $P=\mathrm{Id}$.

Clearly, if $t \leq 0$ or $s \leq r \leq 0 \leq t$ then, thanks to $\left(\mathbf{H}_{2}\right)$, we have that (4.23) holds. If $0 \leq s \leq r \leq t$, recalling that $K \geq 1$, we have that

$$
\exp \left(\int_{s}^{r} \alpha(u) d u\right) \leq N(t) \leq K e^{\alpha_{1} r} N(t) e^{-\alpha_{1}(r-s)} \leq K(t) e^{-\alpha_{1}(r-s)}
$$


Finally, for $s \leq 0 \leq r \leq t$, again from $\left(\mathbf{H}_{2}\right)$ and from the definition of $N(\cdot)$, we have that

$$
\begin{aligned}
\exp \left(\int_{s}^{r} \alpha(u) d u\right) & \leq \exp \left(\int_{s}^{0} \alpha(u) d u+\int_{0}^{r} \alpha(u) d u\right) \\
& \leq K e^{\alpha_{1} s} N(t) \leq K(t) e^{-\alpha_{1}(r-s)}
\end{aligned}
$$

Gathering all the previous facts, we have that (4.23) holds as stated.

Let $D$ be a bounded set. Thus, there is a positive constant $d>0$ such that $\sup _{x \in D}|x| \leq d$. We take $x_{0} \in D, t \in \mathbb{R}, s \geq 0$ and denote by $x(\cdot):=$ $x\left(\cdot, f, t-s, x_{0}\right)$, i.e. the solution of the Cauchy problem $\dot{x}=f(t, x), x(t-s)=x_{0}$. In particular, since $S_{f}(t, t-s) x_{0}=x\left(t, f, t-s, x_{0}\right)=x(t)$ from (4.21) and (4.23) we deduce that

$$
\left|S_{f}(t, t-s) x_{0}\right|^{2} \leq\left|x_{0}\right|^{2} K(t) e^{-\alpha_{1} s}+I(t, s)
$$

where

$$
\begin{aligned}
I(t, s) & =\int_{t-s}^{t} \beta(r) \exp \left(\int_{r}^{t} \alpha(u) d u\right) d r \leq K(t) \int_{-\infty}^{t} e^{-\alpha_{1}(t-r)} \beta(r) d r \\
& =K(t) \int_{-t}^{\infty} e^{-\alpha_{1}(t+u)} \beta(-u) d u .
\end{aligned}
$$

Now, from $\left(\mathbf{H}_{3}\right)$ there is a $c_{1}>0$ such that

$$
\sup _{t \in \mathbb{R}} \int_{t}^{t+1} \beta(u) d u \leq c_{1}
$$

and, hence, since $[-t, \infty) \subset \bigcup_{j=0}^{\infty}[-t+j,-t+j+1]$, we obtain

$$
I(t, s) \leq K(t) \sum_{j=0}^{\infty} \int_{-t+j}^{-t+j+1} e^{-\alpha_{1} j} \beta(-u) d u \leq c_{1} K(t) \sum_{j=0}^{\infty} e^{-\alpha_{1} j} \leq \frac{c_{1} K(t)}{1-e^{-\alpha_{1}}}
$$

because $\alpha_{1}>0$. Therefore, denoting by

$$
\rho^{2}(t):=1+\frac{c_{1} K(t)}{1-e^{-\alpha_{1}}}, \quad T(t, D):=\frac{\ln \left(d^{2} K(t)\right)}{\alpha_{1}},
$$

from (4.24) and (4.26), one has that

$$
\left|S_{f}(t, t-s) x_{0}\right|^{2} \leq d^{2} K(t) e^{-\alpha_{1} s}+I(t, s) \leq \rho^{2}(t),
$$

provided that $s \geq T(t, D)>0$.

Hence, $\left\{B_{\rho(t)} \mid t \in \mathbb{R}\right\}$ is a family of bounded absorbing sets. In addition, since the function $K(\cdot)$, defined in (4.22), is nondecreasing, then $\rho(t)$ and $T(t, D)$ are also nondecreasing. Therefore, we deduce that

$$
S_{f}(t, t-s) D \subset B_{\rho(\tau)} \quad \text { for } t \leq \tau \text { and } s \geq T(\tau, D)
$$

and the process is strongly pullback bounded dissipative on $(-\infty, \tau]$ for all $\tau \in \mathbb{R}$, as claimed. The existence of a pullback attractor bounded in the past follows from Remark 4.6. 
Consequently, an application of Theorem 4.10 provides for each $g$ in the alpha limit set $\mathbb{A}(f)$ the existence of a bounded pullback attractor for the process $S_{g}(\cdot, \cdot)$.

Corollary 4.24. Let $f$ be in $\mathfrak{L} \mathfrak{C}$ and $\mathcal{T}$ be a topology such that the induced local skew-product flow on $\operatorname{Hull}_{(\mathfrak{L C}, \mathcal{T})}(f) \times \mathbb{R}^{N}$ is continuous. Under assumptions $\left(\mathbf{H}_{1}\right)$, $\left(\mathbf{H}_{2}\right)$ and $\left(\mathbf{H}_{3}\right)$, for each $g \in \mathbb{A}(f)$ the solutions of $\dot{x}=g(t, x)$ are uniformly ultimately bounded. In particular, the induced process $S_{g}(\cdot, \cdot)$ has a bounded pullback attractor.

Proof. Firstly, notice that, thanks to Remark 4.22, the solutions of $\dot{x}=f_{s}(t, x)$, with $s \in \mathbb{R}$, are defined on $[0, \infty)$. Therefore, in order to apply Theorem 4.10, we only need to prove that for any $g \in \mathbb{A}(f)$ and any $x_{0} \in \mathbb{R}^{N}$ the solution of $\dot{x}=g(t, x), x(0)=x_{0}$ is defined on $[0, \infty)$. From (4.24) and (4.26) and recalling that the function $K(\cdot)$, defined in (4.22), is nondecreasing, one has that for any $d \geq 0$, and $x_{0} \in \mathbb{R}^{N}$ with $\left|x_{0}\right|<d$

$$
\left|S_{f}(t, t-s) x_{0}\right|=\left|x\left(s, f_{t-s}, x_{0}\right)\right| \leq c(d) \quad \text { for all } t \leq 0, s \geq 0
$$

where $c^{2}(d)=K(0)\left(d^{2}+c_{1} /\left(1-e^{-\alpha_{1}}\right)\right)$ and $c_{1}>0$ is determined by (4.25). Now, consider some $g \in \mathbb{A}(f)$, i.e. $g=\lim _{n \rightarrow \infty} f_{t_{n}}$ with $t_{n} \downarrow-\infty$, and let us fix $s \in\left[0, b_{g, x_{0}}\right)$. Notice that, for any $n \in \mathbb{N}$, one may write $x\left(s, f_{t_{n}}, x_{0}\right)$ as $x\left(s, f_{\left(t_{n}+s\right)-s}, x_{0}\right)$. Thus, considered $n_{0} \in \mathbb{N}$ such that $t_{n}+s \leq 0$ for any $n \geq$ $n_{0}$, one has that $x\left(s, f_{t_{n}}, x_{0}\right)=x\left(s, f_{\left(t_{n}+s\right)-s}, x_{0}\right)$ satisfies (4.27) for any $n \geq n_{0}$ and, by the continuity of the flow, the sequence $\left(x\left(s, f_{t_{n}}, x_{0}\right)\right)_{n \in \mathbb{N}}$ converges to $x\left(s, g, x_{0}\right)$. Therefore, we conclude that

$$
\left|x\left(s, g, x_{0}\right)\right| \leq c(d) \quad \text { for all } s \in\left[0, b_{g, x_{0}}\right) \text { and } x_{0} \in \mathbb{R}^{N} \text { with }\left|x_{0}\right|<d .
$$

As a consequence, one has that the solution $x\left(\cdot, g, x_{0}\right)$ of $\dot{x}=g(s, x), x(0)=x_{0}$ can not explode in finite time, i.e. it has to be defined on $[0, \infty)$. Otherwise it is easy to prove that a contradiction arises.

One concludes the proof applying Theorem 4.10.

In order to have that for all $\tau \in \mathbb{R}$ the solutions of $\dot{x}=f(t, x)$ are uniformly ultimately bounded on $[\tau, \infty)$, we change hypothesis $\left(\mathbf{H}_{2}\right)$ by

$\left(\mathbf{H}_{2}^{*}\right)$ the linear equation $\dot{y}=\alpha(t) y$ has exponential dichotomy on $[0, \infty)$ with projection $P=$ Id, i.e. there is an $\alpha_{1}>0$ and a constant $K \geq 1$ such that

$$
\exp \left(\int_{s}^{t} \alpha(u) d u\right) \leq K e^{-\alpha_{1}(t-s)} \quad \text { for every } 0 \leq s \leq t .
$$

Theorem 4.25. Under conditions $\left(\mathbf{H}_{1}\right),\left(\mathbf{H}_{2}^{*}\right)$ and $\left(\mathbf{H}_{3}\right)$, for each fixed $\tau \in \mathbb{R}$ the solutions of $\dot{x}=f(t, x)$ are uniformly ultimately bounded on $[\tau, \infty)$. 
Proof. Analogously to the first part of the proof of Theorem 4.23, consider now the nonincreasing function $K(\cdot) \geq 1$ defined by

$$
K\left(t_{0}\right):= \begin{cases}K e^{-\alpha_{1} t_{0}} N\left(t_{0}\right) & \text { if } t_{0}<0 \\ K & \text { if } t_{0} \geq 0\end{cases}
$$

where $N\left(t_{0}\right):=\exp \left(\int_{t_{0}}^{0}|\alpha(u)| d u\right)$. First we check that one has

$$
\exp \left(\int_{s}^{r} \alpha(u) d u\right) \leq K\left(t_{0}\right) e^{-\alpha_{1}(r-s)} \quad \text { for every } t_{0} \leq s \leq r,
$$

that is, for any $t_{0} \in \mathbb{R}$, the equation $\dot{y}=\alpha(t) y$ has exponential dichotomy on $\left[t_{0}, \infty\right)$ with projection $P=\mathrm{Id}$.

Clearly, if $t_{0} \geq 0$ or $t_{0} \leq 0 \leq s \leq r$ then, thanks to $\left(\mathbf{H}_{2}^{*}\right)$, we have that (4.29) holds. If $t_{0} \leq s \leq r \leq 0$, then, recalling that $K \geq 1$, we have that

$$
\exp \left(\int_{s}^{r} \alpha(u) d u\right) \leq N\left(t_{0}\right) \leq K\left(t_{0}\right) e^{-\alpha_{1}(r-s)} .
$$

Finally, for $t_{0} \leq s \leq 0 \leq r$, again from $\left(\mathbf{H}_{2}^{*}\right)$ and from the definition of $N(\cdot)$, we have that

$$
\begin{aligned}
\exp \left(\int_{s}^{r} \alpha(u) d u\right) & \leq \exp \left(\int_{s}^{0} \alpha(u) d u+\int_{0}^{r} \alpha(u) d u\right) \\
& \leq N\left(t_{0}\right) K e^{-\alpha_{1} r} \leq K\left(t_{0}\right) e^{-\alpha_{1}(r-s)} .
\end{aligned}
$$

As a consequence we have that (4.29) holds as stated.

Now, consider $d>0$ and let $x_{0} \in \mathbb{R}^{N}$ be such that $\left|x_{0}\right| \leq d$. Recalling that $S_{f}\left(t+t_{0}, t_{0}\right) x_{0}$ represents the solution $x\left(t+t_{0}, f, t_{0}, x_{0}\right)$ of the Cauchy Problem $\dot{x}=f(t, x), x\left(t_{0}\right)=x_{0}$, then, thanks to (4.21) and (4.29), we deduce that

$$
\left|S_{f}\left(t+t_{0}, t_{0}\right) x_{0}\right|^{2} \leq\left|x_{0}\right|^{2} K\left(t_{0}\right) e^{-\alpha_{1} t_{0}}+I\left(t, t_{0}\right), \quad \text { for all } t \geq 0,
$$

where

$$
\begin{aligned}
I\left(t, t_{0}\right) & =\int_{t_{0}}^{t+t_{0}} \beta(r) \exp \left(\int_{r}^{t+t_{0}} \alpha(u) d u\right) d r \leq K\left(t_{0}\right) \int_{t_{0}}^{t+t_{0}} \beta(r) e^{-\alpha_{1}\left(t+t_{0}-r\right)} d r \\
& =K\left(t_{0}\right) \int_{-t_{0}}^{t-t_{0}} \beta(t-u) e^{-\alpha_{1}\left(t_{0}+u\right)} d u \leq K\left(t_{0}\right) \int_{-t_{0}}^{\infty} \beta(t-u) e^{-\alpha_{1}\left(t_{0}+u\right)} d u .
\end{aligned}
$$

Now, recalling that for $\left(\mathbf{H}_{3}\right)$ there exists $c_{1}>0$ is such that

$$
\sup _{t \in \mathbb{R}} \int_{t}^{t+1} \beta(u) d u \leq c_{1},
$$

then, as in Theorem 4.23, we deduce that

$$
I\left(t, t_{0}\right) \leq \frac{c_{1} K\left(t_{0}\right)}{1-e^{-\alpha_{1}}} \quad \text { for all } t \geq 0 .
$$


Thus, denoting by

$$
c^{2}\left(t_{0}\right):=1+\frac{c_{1} K\left(t_{0}\right)}{1-e^{-\alpha_{1}}} \quad \text { and } \quad T\left(t_{0}, d\right):=\frac{\ln \left(d^{2} K\left(t_{0}\right)\right)}{\alpha_{1}},
$$

from (4.30) and (4.31) we have that

$$
\left|S_{f}\left(t+t_{0}, t_{0}\right) x_{0}\right| \leq c\left(t_{0}\right) \quad \text { whenever } t \geq T\left(t_{0}, d\right) \text { and }\left|x_{0}\right| \leq d,
$$

and the nonincreasing character of $c(\cdot)$ and $T(\cdot, d)$ proves $(4.2)$ and finishes the proof.

Hence, an application of Theorem 4.11 provides, for each $g$ in the omega limit set $O(f)$, the existence of a bounded pullback attractor for the induced process $S_{g}(\cdot, \cdot)$.

Corollary 4.26. Let $f$ be in $\mathfrak{L} \mathfrak{C}$ and $\mathcal{T}$ be a topology such that the induced local skew-product flow on $\operatorname{Hull}_{(\mathfrak{L C}, \mathcal{T})}(f) \times \mathbb{R}^{N}$ is continuous and assume that conditions $\left(\mathbf{H}_{1}\right),\left(\mathbf{H}_{2}^{*}\right)$ and $\left(\mathbf{H}_{3}\right)$ hold. Then, for each $g \in \mathbb{O}(f)$ the solutions of $\dot{x}=g(t, x)$ are uniformly ultimately bounded and the induced process $S_{g}(\cdot, \cdot)$ has a bounded pullback attractor.

Proof. Firstly, notice that, thanks to Remark 4.22, the solutions of $\dot{x}=f_{s}(t, x)$, with $s \in \mathbb{R}$, are defined on $[0, \infty)$. Therefore, in order to apply Theorem 4.11, we only need to prove that for any $g \in \mathbb{O}(f)$ and any $x_{0} \in \mathbb{R}^{N}$ the solution of $\dot{x}=g(t, x), x(0)=x_{0}$ is defined on $[0, \infty)$. From (4.30), (4.31) and recalling that the function $K(\cdot)$, defined in (4.28), is nonincreasing, one has that for any $d \geq 0$, and $x_{0} \in \mathbb{R}^{N}$ with $\left|x_{0}\right|<d$

$$
\left|S_{f}\left(t+t_{0}, t_{0}\right) x_{0}\right|=\left|x\left(t, f_{t_{0}}, x_{0}\right)\right| \leq \widetilde{c}(d) \quad \text { for all } t_{0} \in \mathbb{R}, t \geq 0,
$$

where $\widetilde{c}^{2}(d)=K(0)\left(d^{2}+c_{1} /\left(1-e^{-\alpha_{1}}\right)\right)$ and $c_{1}>0$ is determined by (4.25). Now, consider some $g \in \mathbb{O}(f)$, i.e. $g=\lim _{n \rightarrow \infty} f_{t_{n}}$ with $t_{n} \uparrow \infty$, and let us fix $t \in\left[0, b_{g, x_{0}}\right)$. Thus, considered $n_{0} \in \mathbb{N}$ such that $t_{n} \geq 0$ for any $n \geq n_{0}$, one has that $x\left(t, f_{t_{n}}, x_{0}\right)$ satisfies (4.33) for any $n \geq n_{0}$ and, by the continuity of the flow, the sequence $\left(x\left(t, f_{t_{n}}, x_{0}\right)\right)_{n \in \mathbb{N}}$ converges to $x\left(t, g, x_{0}\right)$. Therefore, we conclude that

$$
\left|x\left(t, g, x_{0}\right)\right| \leq \widetilde{c}(d) \quad \text { for all } t \in\left[0, b_{g, x_{0}}\right) \text { and } x_{0} \in \mathbb{R}^{N} \text { with }\left|x_{0}\right|<d .
$$

As a consequence, one has that the solution $x\left(\cdot, g, x_{0}\right)$ of $\dot{x}=g(s, x), x(0)=x_{0}$ can not explode in finite time, i.e. it has to be defined on $[0, \infty)$. Otherwise it is easy to prove that a contradiction arises.

One concludes the proof applying Theorem 4.11 .

Next, substituting $\left(\mathbf{H}_{2}\right)$ with the stronger assumption below, we obtain a pullback bounded absorbing set $B$ satisfying (4.4) which is what we need in the assumptions of Theorem 4.12. 
$\left(\mathbf{H}_{2}^{\bullet}\right)$ the linear equation $\dot{y}=\alpha(t) y$ has exponential dichotomy on $\mathbb{R}$ with projection $P=\mathrm{Id}$, i.e. there is an $\alpha_{1}>0$ and a constant $K \geq 1$ such that

$$
\exp \left(\int_{s}^{t} \alpha(u) d u\right) \leq K e^{-\alpha_{1}(t-s)} \quad \text { for } s \leq t ;
$$

Theorem 4.27. Consider $f \in \mathfrak{L} \mathfrak{C}$ and assume that $\left(\mathbf{H}_{1}\right),\left(\mathbf{H}_{2}^{\bullet}\right)$ and $\left(\mathbf{H}_{3}\right)$ hold. Then there is a pullback bounded absorbing set B satisfying (4.4) and, hence, the induced process $S_{f}(\cdot, \cdot)$ has a bounded pullback attractor.

Proof. From $\left(\mathbf{H}_{2}^{\bullet}\right)$ we have that

$$
\exp \left(\int_{t-s}^{t} \alpha(u) d u\right) \leq K e^{-\alpha_{1} t} \quad \text { for all } t \in \mathbb{R} \text { and } s \geq 0 .
$$

Therefore, reasoning as in Theorem 4.23, with the exception that now $K(\cdot) \equiv K$, one has that

$$
S_{f}(t, t-s) D \subset B_{\rho} \quad \text { for every } t \in \mathbb{R} \text { and } s \geq T(D),
$$

where $\rho^{2}=1+c_{1} K /\left(1-e^{-\alpha_{1}}\right), T(D)=\ln \left(d^{2} K\right) / \alpha_{1}$, and (4.4) holds with $B_{\rho}$, as stated. In particular, $S_{f}(\cdot, \cdot)$ has a bounded pullback attractor.

As a consequence, an application of Theorem 4.12 provides the existence of a bounded pullback attractor for the process $S_{g}(\cdot, \cdot)$ induced by $g \in \operatorname{Hull}_{(\mathfrak{L C}, \mathcal{T})}(f)$.

Corollary 4.28. Let $f$ be in $\mathfrak{L} \mathfrak{C}$ and $\mathcal{T}$ be a topology such that the induced local skew-product flow on $\operatorname{Hull}_{(\mathfrak{L C}, \mathcal{T})}(f) \times \mathbb{R}^{N}$ is continuous and assume that $\left(\mathbf{H}_{1}\right)$, $\left(\mathbf{H}_{2}^{\bullet}\right)$ and $\left(\mathbf{H}_{3}\right)$ hold. Then if $g \in \operatorname{Hull}_{(\mathfrak{L C}, \mathcal{T})}(f)$ one has that the solutions of $\dot{x}=g(t, x)$ are uniformly ultimately bounded, and the induced process $S_{g}(\cdot, \cdot)$ has a bounded pullback attractor.

Proof. In order to apply Theorem 4.12, we need to prove that for any $g \in$ $\operatorname{Hull}_{(\mathfrak{L C}, \mathcal{T})}(f)$ and any $x_{0} \in \mathbb{R}^{N}$ the solution of $\dot{x}=g(t, x), x(0)=x_{0}$ is defined on $[0, \infty)$. Reasoning as in Theorem 4.23, with the exception that now, since (4.35) holds, we can pose $K(\cdot) \equiv K$, from (4.24) and (4.26), one has that

$$
\left|S_{f}(t, t-s) x_{0}\right|^{2} \leq\left|x_{0}\right|^{2} K e^{-\alpha_{1} s}+\frac{c_{1} K}{1-e^{-\alpha_{1}}} .
$$

where $c_{1}>0$ is determined by (4.25). In other words, we can write

$$
\left|S_{f}(t, t-s) x_{0}\right|=\left|x\left(s, f_{t-s}, x_{0}\right)\right| \leq \widetilde{c}(d) \quad \text { for all } t \in \mathbb{R} \text { and } s \geq 0,
$$

where $\widetilde{c}^{2}(d)=K\left(d^{2}+c_{1} /\left(1-e^{-\alpha_{1}}\right)\right)$. Now, reasoning as in either Remark 4.22, Corollary 4.24 or Corollary 4.26, depending on the fact that, respectively, $g=f_{\tau}$ for some $\tau \in \mathbb{R}, g \in \mathbb{A}(f)$ or $g \in \mathbb{O}(f)$, one obtains that the solution of $\dot{x}=$ $g(t, x), x(0)=x_{0}$ is defined on $[0, \infty)$.

One concludes the proof applying Theorem 4.10. 
We summarize the results for the existence of a pullback and a global attractor for the induced skew-product semiflow in the following remark.

Remark 4.29. Under assumptions $\left(\mathbf{H}_{1}\right),\left(\mathbf{H}_{2}^{\bullet}\right)$ and $\left(\mathbf{H}_{3}\right)$, (i) and (ii) of Theorem 4.13 hold. The same happens for Corollary 4.16 (resp. 4.17) when $\left(\mathbf{H}_{1}\right),\left(\mathbf{H}_{2}\right)$ and $\left(\mathbf{H}_{3}\right)$ (resp. $\left(\mathbf{H}_{1}\right),\left(\mathbf{H}_{2}^{*}\right)$ and $\left.\left(\mathbf{H}_{3}\right)\right)$ are assumed.

As follows we present three simple examples to show the applicability of the previous theorems.

Example 4.30. Consider the system

$$
\dot{x}=f(t, x)=a(t) x, \quad \text { with } a(t)=\left\{\begin{aligned}
-1 & \text { if } t \leq 0 \\
1 & \text { if } t>0
\end{aligned}\right.
$$

Such a system trivially satisfies $\left(\mathbf{H}_{1}\right),\left(\mathbf{H}_{2}\right)$ and $\left(\mathbf{H}_{3}\right)$, and thus the induced process, $S_{f}(\cdot, \cdot)$ is strongly pullback dissipative on $(-\infty, \tau]$ for all $\tau \in \mathbb{R}$. As a consequence, the system admits a pullback attractor which is bounded in the past. In fact, the pullback attractor for $S_{f}(\cdot, \cdot)$ is given by

$$
\mathcal{A}(t)=0, \quad \text { for all } t \in \mathbb{R} .
$$

Indeed, for any $x_{0} \in \mathbb{R}^{N}, s \leq 0$ and any fixed $t \in \mathbb{R}$ one has

$$
\left|S_{f}(t, s) x_{0}\right|=\left|x_{0}\right| \exp \left(\int_{s}^{t} a(u) d u\right)= \begin{cases}\left|x_{0}\right| e^{s-t} & \text { if } t \leq 0, \\ \left|x_{0}\right| e^{s+t} & \text { if } t>0\end{cases}
$$

which goes to zero as $s \rightarrow-\infty$. Notice also that the system does not have any forward attractor since for any fixed $s \in \mathbb{R}$ one has that $\left|S_{f}(t, s)\right| \rightarrow \infty$ as $t \rightarrow \infty$. Finally, notice that $\mathbb{A}(f)=\{-x\}$, and again the set given by $\mathcal{A}(t)=\{0\}$ for all $t \in \mathbb{R}$, is the (bounded) pullback attractor for the process induced by the system $\dot{x}=-x$. However, in this case, $\{0\}$ is also a forward attractor for $\dot{x}=-x$.

Example 4.31. Consider the system

$$
\dot{x}=f(t, x)=a(t) x+1
$$

where $a(\cdot)$ is the one given in (4.37). The induced process is

$$
S_{f}(t, s) x_{0}=x_{0} \exp \left(\int_{s}^{t} a(u) d u\right)+\int_{s}^{t} \exp \left(\int_{r}^{t} a(u) d u\right) d r .
$$

Notice that the system trivially satisfies $\left(\mathbf{H}_{1}\right),\left(\mathbf{H}_{2}\right)$ and $\left(\mathbf{H}_{3}\right)$ and in fact we obtain a pullback attractor which is bounded in the past. In particular, using the definition of $a(\cdot)$, if $t \leq 0$, then

$$
S_{f}(t, s) x_{0}=x_{0} e^{s-t}+1-e^{s-t}
$$


which goes to 1 as $s \rightarrow-\infty$. As a consequence, we have that $\mathcal{A}(t)=1$ for all $t \leq 0$. On the other hand, by the invariance of the pullback attractor, we have that for any $t>0$

$$
\mathcal{A}(t)=S_{f}(t, 0) \mathcal{A}(0)=x(t, f, 1),
$$

that is, the solution of (4.38) starting at $x(0)=1$ which is

$$
x(t, f, 1)=-1+2 e^{t} .
$$

Summing up, the pullback attractor for this system is given by

$$
\mathcal{A}(t)=\left\{\begin{array}{cc}
1 & \text { if } t \leq 0 \\
-1+2 e^{t} & \text { if } t>0
\end{array}\right.
$$

and notice that $\bigcup_{t \in \mathbb{R}} \mathcal{A}(t)$ is not bounded. Finally, one has that $\mathbb{A}(f)=\{-x+1\}$ and the set given by $\mathcal{A}(t)=\{1\}$ for all $t \in \mathbb{R}$, is the bounded pullback attractor for the process induced by the system $\dot{x}=-x+1$.

Example 4.32. Consider the system

$$
\dot{x}=f(t, x)=a(t) x, \quad \text { with } a(t)=\left\{\begin{aligned}
1 & \text { if } t \leq 0 \\
-1 & \text { if } t>0
\end{aligned}\right.
$$

Such a system trivially satisfies $\left(\mathbf{H}_{1}\right),\left(\mathbf{H}_{2}^{*}\right)$ and $\left(\mathbf{H}_{3}\right)$, and thus, for each fixed $\tau \in \mathbb{R}$ the solutions of $\dot{x}=f(t, x)$ are uniformly ultimately bounded on $[\tau, \infty)$. In fact, we have that

$$
\left|S_{f}(t, s) x_{0}\right|=\left|x_{0}\right| \exp \left(\int_{s}^{t} a(u) d u\right)= \begin{cases}\left|x_{0}\right| e^{t-s} & \text { if } s \leq t \leq 0 \\ \left|x_{0}\right| e^{-s-t} & \text { if } s \leq 0<t \\ \left|x_{0}\right| e^{s-t} & \text { if } 0 \leq s \leq t\end{cases}
$$

where $S_{f}(\cdot, \cdot)$ denotes the process induced by (4.39), and one has that

$$
\lim _{t \rightarrow \infty} S_{f}(t, s) x=0, \quad \text { for all } x \in \mathbb{R}^{N} \text { and } s \in \mathbb{R} .
$$

Notice also that such system does not admit a pullback attractor. In fact for any fixed $t \in \mathbb{R}$ one has that

$$
\lim _{s \rightarrow-\infty} S_{f}(t, s) x=\infty, \quad \text { for all } x \in \mathbb{R}^{N}
$$

Finally, $\mathbb{O}(f)=\{-x\}$ and the set given by $\mathcal{A}(t)=\{0\}$ for all $t \in \mathbb{R}$, is the (bounded) pullback attractor for the process induced by the system $\dot{x}=-x$. 


\subsubsection{Comparison with a Carathéodory linear system}

In this subsection we aim to develop results analogous to the ones proved in Subsection 4.3.1 but the Carathéodory vector field $f$ is now compared with a whole system of linear Carathéodory equations. Let us firstly recall some notation. For every $i=1, \ldots, N$ the $i$ th component of $x \in \mathbb{R}^{N}$ will be denoted by $x_{i}$. Moreover, if we write $x \geq 0$ we mean that for all $i=1, \ldots, N$ one has $x_{i} \geq 0$, whereas we will write $x \gg 0$ if for every $i=1, \ldots N$ one has $x_{i}>0$. The space $\left(\mathbb{R}^{N}\right)^{+}$will denote the set of points $x \in \mathbb{R}^{N}$ such that $x \geq 0$. Analogously, the $i$ th component of a vector function $f: \mathbb{R} \times \mathbb{R}^{N} \rightarrow \mathbb{R}^{N}$ will be denoted by $f_{i}$.

We consider the new assumptions for $f \in \mathfrak{L} \mathfrak{C}$ :

$\left(\mathbf{A}_{1}\right)$ if $x \geq 0$ with $x_{i}=0$, then $f_{i}(t, x) \geq 0$ for a.e. $t \in \mathbb{R}$;

$\left(\mathbf{A}_{2}\right)$ for a.e $(t, x) \in \mathbb{R} \times\left(\mathbb{R}^{N}\right)^{+}$

$$
f(t, x) \leq A(t) x+b(t)
$$

where the functions $A(\cdot)=\left[a_{i j}(\cdot)\right] \in L_{l o c}^{1}\left(\mathbb{R}^{N \times N}\right), a_{i j}(\cdot) \geq 0$ for every $i \neq j$, $b(\cdot) \in L_{\text {loc }}^{1}\left(\mathbb{R}^{N}\right)$, and $b(t) \geq 0$ for every $t \in \mathbb{R}$;

$\left(\mathbf{A}_{3}\right)$ the linear equation $\dot{y}=A(t) y$ has exponential dichotomy on $(-\infty, 0]$ with projection $P=\mathrm{Id}$, i.e. there is an $\alpha_{1}>0$ and a constant $K \geq 1$ such that

$$
\left\|\Phi(t) \Phi^{-1}(s)\right\| \leq K e^{-\alpha_{1}(t-s)} \quad \text { for } s \leq t \leq 0,
$$

where $\Phi(t)$ is the fundamental matrix solution with $\Phi(0)=\mathrm{I}_{N}$;

$\left(\mathbf{A}_{4}\right)$ the set of functions $\left\{b_{t}(\cdot)\right\}_{t \in \mathbb{R}}$ is $L_{\text {loc }}^{1}\left(\mathbb{R}^{N}\right)$-bounded.

The inequality in $\left(\mathbf{A}_{2}\right)$ actually holds for the positive solutions of $\dot{x}=f(t, x)$. The proof is similar to the one of Proposition 4.21 and thus omitted.

Proposition 4.33. Let $f$ be a function in $\mathfrak{L} \mathfrak{C}$ satisfying $\left(\mathbf{A}_{2}\right)$. If $x(t)$ is a solution of $\dot{x}=f(t, x)$ defined on an interval $I$, with $x(t) \geq 0$ for all $t \in I$, then

$$
f(t, x(t)) \leq A(t) x(t)+b(t) \quad \text { for a.e. } t \in I .
$$

Conditions $\left(\mathbf{A}_{1}\right)$ and $\left(\mathbf{A}_{2}\right)$ imply that the system $\dot{x}=f(t, x)$ induces a continuous time process on $\left(\mathbb{R}^{N}\right)^{+}$, as shown in the following result.

Proposition 4.34. Let $f$ be a function in $\mathfrak{L} \mathfrak{C}$ and $x\left(t, f, t_{0}, x_{0}\right)$ the solution of $\dot{x}=f(t, x), x\left(t_{0}\right)=x_{0}$ with $x_{0} \geq 0$.

(i) If $f$ satisfies $\left(\mathbf{A}_{1}\right)$, then $x\left(t, f, t_{0}, x_{0}\right) \geq 0$ for every $t \geq t_{0}$ on its maximal interval of existence. 
(ii) If $f$ satisfies $\left(\mathbf{A}_{1}\right)$ and $\left(\mathbf{A}_{2}\right)$, then $x\left(t, f, t_{0}, x_{0}\right)$ is defined on $\left[t_{0}, \infty\right)$.

As a consequence, under assumptions $\left(\mathbf{A}_{1}\right)$ and $\left(\mathbf{A}_{2}\right)$ a continuous time process is induced on $\left(\mathbb{R}^{N}\right)^{+}$by

$$
S_{f}(t, s) x_{0}=x\left(t, f, s, x_{0}\right)=x\left(t-s, f_{s}, x_{0}\right) \geq 0, \quad \forall t \geq s \text { and } x_{0} \in\left(\mathbb{R}^{N}\right)^{+} .
$$

Proof. (i) Denoting by 0 the $N$-dimensional null vector, by $\left(\mathbf{A}_{1}\right)$, we have

$$
0 \leq f(t, x) \quad \text { for all } x \in\left(\mathbb{R}^{N}\right)^{+} \text {and a.e. } t \in \mathbb{R} \text {. }
$$

Therefore, applying Proposition 4.19 to $z(\cdot) \equiv 0$, we deduce that for any $x_{0} \geq 0$

$$
0 \leq x\left(t, f, t_{0}, x_{0}\right)
$$

for all $t \geq t_{0}$ in the maximal interval of existence of $x\left(\cdot, f, t_{0}, x_{0}\right)$, which ends the proof of part (i).

(ii) For simplicity of notation, let $x(t)=x\left(t, f, t_{0}, x_{0}\right)$. From (4.40) we have

$$
\dot{x}(t) \leq A(t) x(t)+b(t) \quad \text { for a.e. } t \in \mathbb{R} .
$$

Thus, since $a_{i j}(\cdot) \geq 0$ for $i \neq j$, the linear system $\dot{y}=A(t) y+b(t)$ is quasimonotone and the assumptions of Proposition 4.19 are satisfied. Therefore, using also (i), one has that $0 \leq x(t) \leq y(t)$ for every $t \in\left(t_{0}, t_{1}\right)$ where $y(t)$ denotes the solution of $\dot{y}=A(t) y+b(t)$ with initial data $y\left(t_{0}\right)=x_{0}$, that is,

$$
0 \leq x(t) \leq \Phi(t) \Phi^{-1}\left(t_{0}\right) x_{0}+\int_{t_{0}}^{t} \Phi(t) \Phi^{-1}(r) b(r) d r,
$$

where the right-hand side comes from the variation of constants formula for Carathéodory linear systems (see [6, Theorem 2.10 p.58]). Therefore, we have that $x(t)=x\left(t, f, t_{0}, x_{0}\right)$ is defined on $\left[t_{0}, \infty\right)$, which concludes part (ii).

The following result provides the existence of a pullback attractor bounded in the past in $\left(\mathbb{R}^{N}\right)^{+}$.

Theorem 4.35. Let $f$ be a function in $\mathfrak{L} \mathfrak{C}$ satisfying $\left(\mathbf{A}_{1}\right),\left(\mathbf{A}_{2}\right),\left(\mathbf{A}_{3}\right)$ and $\left(\mathbf{A}_{4}\right)$. Then, the induced process (4.41) is strongly pullback bounded dissipative on $(-\infty, \tau]$ for all $\tau \in \mathbb{R}$ and, as a consequence, there exists a pullback attractor which is bounded in the past.

Proof. As is Theorem 4.23, from $\left(\mathbf{A}_{3}\right)$ we deduce the existence of exponential dichotomy on $(-\infty, t]$ for any fixed $t \geq 0$. More precisely, there is a nondecreasing function $K(\cdot) \geq 1$ such that

$$
\left\|\Phi(r) \Phi^{-1}(s)\right\| \leq K(t) e^{-\alpha_{1}(r-s)} \quad \text { for } s \leq r \leq t .
$$


Let $D$ be a bounded set of $\left(\mathbb{R}^{N}\right)^{+}$. Thus, there is a positive constant $d>0$ such that $\sup _{x \in D}|x| \leq d$. We take $x_{0} \in D, s \geq 0$ and consider $x(r):=x\left(r, f, t-s, x_{0}\right)$, i.e. the solution of the Cauchy problem $\dot{x}(r)=f(r, x(r)), x(t-s)=x_{0}$. As in Proposition 4.34, we deduce that $0 \leq x(r) \leq y(r)$ for every $r \in[t-s, t]$ where $y(r)$ denotes the solution of $\dot{y}=A(r) y+b(r)$ with initial data $y(t-s)=x_{0}$, that is,

$$
0 \leq x(t) \leq \Phi(t) \Phi^{-1}(t-s) x_{0}+\int_{t-s}^{t} \Phi(t) \Phi^{-1}(r) b(r) d r .
$$

Therefore, inequality (4.42) provides

$$
\left|S_{f}(t, t-s) x_{0}\right| \leq\left|x_{0}\right| K(t) e^{-\alpha_{1} s}+K(t) \int_{t-s}^{t} e^{-\alpha_{1}(t-r)}|b(r)| d r,
$$

and the rest of the proof follows step by step the one of Theorem 4.23 and thus it is omitted.

Remark 4.36. The part in condition $\left(\mathbf{A}_{2}\right)$ which implies that the system $\dot{y}=$ $A(r) y+b(r)$ is quasi monotone, i.e. $a_{i j}(\cdot) \geq 0$ for $i \neq j$, can be substituted by the quasi monotone condition for $\dot{x}=f(r, x)$, that is (4.13). In this case, maintaining the notation of Theorem 4.35 , we would obtain

$$
f(r, y(r)) \leq \dot{y}(r) \quad \text { for a.e. } r
$$

which implies from Remark 4.20 that $x(r) \leq y(r)$ for every $r \in[t-s, t]$, and the rest of the proof remains the same.

From Theorems 4.35 and 4.10 we obtain we following result, whose proof is omitted because it is analogous to the one of Corollary 4.24 .

Corollary 4.37. Let $f$ be a function in $\mathfrak{L} \mathfrak{C}^{\mathfrak{a}}$ and $\mathcal{T}$ be a topology such that the induced skew-product flow on $\operatorname{Hull}_{(\mathfrak{L C}, \mathcal{T})}(f) \times\left(\mathbb{R}^{N}\right)^{+}$is continuous. If $f$ satisfies $\left(\mathbf{A}_{1}\right),\left(\mathbf{A}_{2}\right),\left(\mathbf{A}_{3}\right)$ and $\left(\mathbf{A}_{4}\right)$, and $g \in \mathbb{A}(f)$, then the solutions of $\dot{x}=g(t, x)$ are uniformly ultimately bounded, and the induced process $S_{g}(\cdot, \cdot)$ on $\left(\mathbb{R}^{N}\right)^{+}$has a bounded pullback attractor.

If we change hypothesis $\left(\mathbf{A}_{3}\right)$ by

$\left(\mathbf{A}_{3}^{*}\right)$ the linear equation $\dot{y}=A(t) y$ has exponential dichotomy on $[0, \infty)$ with projection $P=\mathrm{Id}$, i.e. there is an $\alpha_{1}>0$ and a constant $K \geq 1$ such that

$$
\left\|\Phi(t) \Phi^{-1}(s)\right\| \leq K e^{-\alpha_{1}(t-s)} \quad \text { for } 0 \leq s \leq t,
$$

where $\Phi(t)$ is the fundamental matrix solution with $\Phi(0)=\mathrm{I}_{N}$,

we obtain a result analogous to Theorem 4.25.

Theorem 4.38. Let $f$ be a function in $\mathfrak{L} \mathfrak{C}$ satisfying $\left(\mathbf{A}_{1}\right),\left(\mathbf{A}_{2}\right),\left(\mathbf{A}_{3}^{*}\right)$ and $\left(\mathbf{A}_{4}\right)$, for each fixed $\tau \in \mathbb{R}$ the solutions are uniformly ultimately bounded on $[\tau, \infty)$. 
Proof. As is Theorem 4.25, from $\left(\mathbf{A}_{3}^{*}\right)$ we deduce the existence of exponential dichotomy on $\left[t_{0}, \infty\right)$ for any fixed $t_{0} \in \mathbb{R}$. More precisely, there is a nonincreasing function $K(\cdot) \geq 1$ such that

$$
\left\|\Phi(r) \Phi^{-1}(s)\right\| \leq K\left(t_{0}\right) e^{-\alpha_{1}(r-s)} \quad \text { for } t_{0} \leq s \leq r .
$$

Let $x_{0} \in\left(\mathbb{R}^{N}\right)^{+}$be such that $\left|x_{0}\right| \leq d$ for some $d>0$, and consider $x(r):=$ $x\left(r, f, t_{0}, x_{0}\right)$, i.e. the solution of the Cauchy problem $\dot{x}=f(r, x), x\left(t_{0}\right)=x_{0}$. As in Proposition 4.34, we deduce that $0 \leq x(r) \leq y(r)$ for every $r \in\left[t_{0}, t\right]$ where $y(r)$ denotes the solution of $\dot{y}=A(r) y+b(r)$ with initial data $y\left(t_{0}\right)=x_{0}$, that is,

$$
0 \leq x(t) \leq \Phi(t) \Phi^{-1}\left(t_{0}\right) x_{0}+\int_{t_{0}}^{t} \Phi(t) \Phi^{-1}(r) b(r) d r .
$$

Therefore, inequality (4.43) and $x\left(t+t_{0}\right)=S_{f}\left(t+t_{0}, t_{0}\right) x_{0}$ provides

$$
\left|S_{f}\left(t+t_{0}, t_{0}\right) x_{0}\right| \leq\left|x_{0}\right| K\left(t_{0}\right) e^{-\alpha_{1} t_{0}}+K\left(t_{0}\right) \int_{t_{0}}^{t+t_{0}} e^{-\alpha_{1}\left(t+t_{0}-r\right)}|b(r)| d r
$$

and the rest of the proof follows step by step the one of Theorem 4.25 and thus it is omitted.

In particular, this implies that Theorem 4.11 holds in this case and we deduce the following result.

Corollary 4.39. Let $f$ be a function in $\mathfrak{L} \mathfrak{C}$ and $\mathcal{T}$ be a topology such that the induced skew-product flow on $\operatorname{Hull}_{(\mathfrak{L C}, \mathcal{T})}(f) \times\left(\mathbb{R}^{N}\right)^{+}$is continuous. If $f$ satisfies conditions $\left(\mathbf{A}_{1}\right),\left(\mathbf{A}_{2}\right),\left(\mathbf{A}_{3}^{*}\right)$ and $\left(\mathbf{A}_{4}\right)$ and $g \in \mathbb{O}(f)$, then the solutions of $\dot{x}=$ $g(t, x)$ are uniformly ultimately bounded, and the induced process $S_{g}(\cdot, \cdot)$ on $\left(\mathbb{R}^{N}\right)^{+}$ has a bounded pullback attractor.

Finally, if we change hypothesis $\left(\mathbf{A}_{3}\right)$ by

$\left(\mathbf{A}_{3}^{\bullet}\right)$ the linear equation $\dot{y}=A(t) y$ has exponential dichotomy on $\mathbb{R}$ with projection $P=\mathrm{Id}$, i.e. there is an $\alpha_{1}>0$ and a constant $K \geq 1$ such that

$$
\left\|\Phi(t) \Phi^{-1}(s)\right\| \leq K e^{-\alpha_{1}(t-s)} \quad \text { for } s \leq t
$$

where $\Phi(t)$ is the fundamental matrix solution with $\Phi(0)=\mathrm{I}_{N}$,

we obtain a result analogous to Theorem 4.27, whose proof is omitted, and the corresponding corollary, consequence of Theorem 4.12.

Theorem 4.40. Consider $f \in \mathfrak{L} \mathfrak{C}$ satisfying $\left(\mathbf{A}_{1}\right),\left(\mathbf{A}_{2}\right),\left(\mathbf{A}_{3}^{\bullet}\right)$ and $\left(\mathbf{A}_{4}\right)$. Then, there is a pullback bounded absorbing set B satisfying (4.4) and, hence, the induced process (4.41) has a bounded pullback attractor. 
Corollary 4.41. Let $f$ be a function in $\mathfrak{L} \mathfrak{C}$ and $\mathcal{T}$ be a topology such that the induced local skew-product flow on $\operatorname{Hull}_{(\mathfrak{L C}, \mathcal{T})}(f) \times\left(\mathbb{R}^{N}\right)^{+}$is continuous. If $f$ satisfies $\left(\mathbf{A}_{1}\right),\left(\mathbf{A}_{2}\right),\left(\mathbf{A}_{3}^{\bullet}\right)$ and $\left(\mathbf{A}_{4}\right)$, and $g \in \operatorname{Hull}_{(\mathfrak{L C}, \mathcal{T})}(f)$, then the induced process $S_{g}(\cdot, \cdot)$ has a bounded pullback attractor.

Again, we summarize the results for the existence of a pullback and a global attractor for the induced skew-product semiflow on $\operatorname{Hull}_{(\mathfrak{L C}, \mathcal{T})}(f) \times\left(\mathbb{R}^{N}\right)^{+}$(resp. $\mathbb{A}(f) \times\left(\mathbb{R}^{N}\right)^{+}$and $\left.\mathbb{O}(f) \times\left(\mathbb{R}^{N}\right)^{+}\right)$in the following remark.

Remark 4.42. Under assumptions $\left(\mathbf{A}_{1}\right),\left(\mathbf{A}_{2}\right),\left(\mathbf{A}_{3}\right)$ and $\left(\mathbf{A}_{4}\right)$, (i) and (ii) of Theorem 4.13 hold. The same happens for the conclusions of Corollary 4.16 (resp. 4.17) when $\left(\mathbf{A}_{1}\right),\left(\mathbf{A}_{2}\right),\left(\mathbf{A}_{3}\right)$ and $\left(\mathbf{A}_{4}\right)\left(\operatorname{resp} .\left(\mathbf{A}_{1}\right),\left(\mathbf{A}_{2}\right),\left(\mathbf{A}_{3}^{*}\right)\right.$ and $\left.\left(\mathbf{A}_{4}\right)\right)$ are assumed.

Example 4.43. Let us go back to the compartmental modelization presented in Section 3.4.3. Consider $B: I \times \mathbb{R}^{d} \rightarrow \mathbb{R}^{d \times d}$ and $s: I \times \mathbb{R}^{d} \rightarrow\left(\mathbb{R}^{+}\right)^{d}$ satisfying Lipschitz Carathéodory conditions and defining the compartmental system

$$
\dot{x}=f(t, x):=B(t, x) x+s(t, x) .
$$

Therefore, we know that the compartmental conditions hold for $B$. In particular we have that for all $t \in I, x \in \mathbb{R}^{d}$ one has

$$
\begin{aligned}
& -b_{i i}(t, x)<0 \quad \forall i=1, \ldots, n, \\
& -b_{i j}(t, x) \geq 0 \quad \forall i \neq j .
\end{aligned}
$$

As a consequence, for any $x \geq 0$ and such that $x_{i}=0$ for some $i=1, \ldots, N$, we have that

$$
\sum_{j=1}^{N} b_{i j}(t, x) x_{j}+s_{i}(t, x) \geq 0
$$

since the only negative entry on the $i$ th row of $B$ is $b_{i i}$, which is multiplied by zero, and $s(t, x) \geq 0$ by definition. Hence, any compartmental model satisfies condition $\left(\mathbf{A}_{1}\right)$.

If there exist functions $C(\cdot)=\left[c_{i j}(\cdot)\right] \in L_{l o c}^{1}\left(\mathbb{R}^{N \times N}\right), c_{i j}(\cdot) \geq 0$ for every $i \neq j$, $b(\cdot) \in L_{l o c}^{1}\left(\mathbb{R}^{N}\right)$, and $b(t) \geq 0$ for every $t \in \mathbb{R}$ such that

- for a.e $(t, x) \in \mathbb{R} \times\left(\mathbb{R}^{N}\right)^{+}$one has

$$
f(t, x)=B(t, x) x+s(t, x) \leq C(t) x+b(t),
$$

- the linear equation $\dot{y}=C(t) y$ has exponential dichotomy on $(-\infty, 0]$ with projection $P=\mathrm{Id}$, and

- the set of functions $\left\{b_{t}(\cdot)\right\}_{t \in \mathbb{R}}$ is $L_{l o c}^{1}\left(\mathbb{R}^{N}\right)$-bounded, 
then, $f$ satisfies also $\left(\mathbf{A}_{2}\right),\left(\mathbf{A}_{3}\right)$ and $\left(\mathbf{A}_{4}\right)$. Thus, Theorem 4.35 provides the existence of a pullback attractor bounded in the past for the process induced by (4.44), whereas Corollary 4.37 provides a bounded pullback attractor for any process induced by a system whose vector field is in $\mathbb{A}(f)$. On the other hand, if $f$ itself is in the alpha limit set of a Lipschitz Carathéodory function satisfying $\left(\mathbf{A}_{1}\right),\left(\mathbf{A}_{2}\right),\left(\mathbf{A}_{3}\right)$ and $\left(\mathbf{A}_{4}\right)$, then the process induced by (4.44) has a bounded pullback attractor.

Similar reasonings hold in case the system $\dot{y}=C(t) y$ has exponential dichotomy on $[0, \infty)$ (resp. on $\mathbb{R}$ ) with projection $P=\mathrm{Id}$ and applying Theorem 4.38 and Corollary 4.39 (resp. Theorem 4.40 and Corollary 4.41). 


\section{Chapter 5}

\section{Continuity of the semiflow for Carathéodory DDEs}

This chapter aims to extend part of the theory that has been developed so far, to the case of Carathéodory delay differential equations with constant delay of the type

$$
\dot{x}=f(t, x(t), x(t-\tau)),
$$

i.e. in which the vector field not only depends on the time and on the present state, but also on the past.

It is important to notice that such extension is not trivial because steps into an infinite-dimensional formulation. In fact the phase space for a skew-product flow induced by (5.1) would be $C\left([-\tau, 0], \mathbb{R}^{N}\right)$. Nevertheless, the specific type of differential equations considered, together with the fact that the function $f$ is defined on a finite-dimensional space, make it possible to use some of the arguments of the previous chapters to extend or develop, with unavoidable discrepancies, some of the results seen before.

An equation like (5.1) allows to modelize a variety of phenomena in which the past consistently influences the future. One may object that, however, this is the simplest type of dependence on the past that one can think of. Yet, this formulation finds extensive and successful application in the modelization of many phenomena. Examples include applications in economics, industry, military problems, population dynamics and more (see Bellman and Danskin [8] and Smith [56] for further details).

The chapter is structured as follows. In Section 5.1, we provide basic preliminaries of Carathéodory delay differential equations with constant delay.

In Section 5.2, we introduce new strong and weak topologies which exhibit a hybrid behavior with respect to the ones presented in Chapter 2. Furthermore, we show how such topologies relate to the first ones and how to apply or develop, in this new context, some of the topological results contained in Sections 2.2, 2.3, 2.4 and 2.5 . 
Section 5.3 deals with the continuity of the skew-product semiflow induced by problems of the type (5.1) when $\mathfrak{L} \mathfrak{C}$ is endowed with either $\mathcal{T}_{B}, \mathcal{T}_{D}$ or the new hybrid topologies $\mathcal{T}_{\Theta B}, \mathcal{T}_{\Theta D}$ and $\sigma_{\Theta D}$ (and suitable hypothesis are assumed on $f$ ).

\subsection{Preliminaries}

For the sake of completeness and to set some notation, we include a theorem of existence and uniqueness of the solution for a Cauchy Problem of Carathéodory type with constant delay. A proof can be derived by the one given for Carathéodory ordinary differential equations in [21, Theorem 1.1, p.43, Theorem 1.2, p.45 and Theorem 2.2, p.49] as shown below (see Cruz and Hale [22] and Hale and Verduyn Lunel [29] for the proofs of existence, uniqueness and continuous dependence for a more general class of delay differential equations). Notice also that, in order to simplify the notation, from now on we will consider the delay $\tau=1$ so that (5.1) takes the form

$$
\dot{x}=f(t, x(t), x(t-1)) .
$$

Moreover, we will denote by $\mathcal{C}$ the set

$$
\mathcal{C}:=C\left([-1,0], \mathbb{R}^{N}\right) .
$$

Theorem 5.1. For any $f \in \mathfrak{L} \mathfrak{C}$ and any $\phi \in \mathcal{C}$ there exists a maximal interval $I_{f, \phi}=\left(-1, b_{f, \phi}\right)$ and a unique continuous function $x(\cdot, f, \phi)$ defined on $I_{f, \phi}$ which is the solution of the delay differential problem

$$
\left\{\begin{array}{l}
\dot{x}=f(t, x(t), x(t-1)), \\
x(t)=\phi(t) \quad \text { for } t \in[-1,0] .
\end{array}\right.
$$

In particular, if $b_{f, \phi}<\infty$, then $|x(t, f, \phi)| \rightarrow \infty$ as $t \rightarrow b_{f, \phi}$.

Proof. Firstly, consider the ordinary differential Cauchy Problem

$$
\left\{\begin{array}{l}
\dot{x}=f(t, x(t), \phi(t-1)), \\
x(0)=\phi(0) .
\end{array}\right.
$$

Due to Theorem 1.2, such problem admits a solution $\widetilde{x}_{0}(\cdot)$ defined on $\left[0, b_{f, \phi(0)}\right)$, where $b_{f, \phi(0)}$ is as in Theorem 1.2. In particular, $\widetilde{x}_{0}(\cdot)$ satisfies $(5.2)$ on $\left[0, T_{0}\right]$ for any $0 \leq T_{0}<\min \left\{1, b_{f, \phi(0)}\right\}$. Therefore, defining

$$
\widetilde{x}_{0}(t):=\phi(t) \text { for } t \in[-1,0],
$$

we have that $\widetilde{x}_{0}(\cdot)$ is a solution of $(5.2)$ on $\left[-1, T_{0}\right]$. If in particular $1<b_{f, \phi(0)}$, then we can repeat the same reasoning in the interval $\left[0, T_{1}\right]$, with $1 \leq T_{1}<$ $\min \left\{2, b_{f, \widetilde{x}_{0}(1)}\right\}$ for the delay differential problem

$$
\left\{\begin{array}{l}
\dot{x}=f(t, x(t), x(t-1)), \\
x(t)=\widetilde{x}_{0}(t) \quad \text { for } t \in[0,1]
\end{array}\right.
$$


and obtain the solution $\widetilde{x}_{1}(\cdot)$ defined in $\left[0, T_{1}\right]$. In particular, due to the uniqueness of the solution for the ordinary differential Cauchy Problems provided by Theorem 1.2 , we can extend $\widetilde{x}_{0}(\cdot)$ to $\left[-1, T_{1}\right]$ by posing $\widetilde{x}_{0}(t):=\widetilde{x}_{1}(t)$ for all $t \in\left[T_{0}, T_{1}\right]$ which is the solution of $(5.2)$ on $\left[-1, T_{1}\right]$. Reiterating such argument we end up with a solution of (5.2) in a maximal interval $\left[-1, b_{f, \phi}\right)$.

\subsection{Spaces and topologies revised}

In this section we aim to get back to the spaces introduced in section 2 and blend them in the context of delay differential equations. While still using the same spaces of functions as before, in the following we will slightly change the notation on such spaces (to avoid any possible misunderstanding) by including the set where the space variable takes values. For example, if $f: \mathbb{R} \times \mathbb{R}^{2 N} \rightarrow \mathbb{R}^{N}$ satisfies (C1) and (C2), we will write that $f \in \mathfrak{C}\left(\mathbb{R}^{2 N}, \mathbb{R}^{N}\right)$ (instead of just $\left.f \in \mathfrak{C}\left(\mathbb{R}^{N}\right)\right)$. The same principle holds also for the rest of the spaces.

An important role will be played by an hybrid version of the topologies presented in Chapter 2. The term hybrid means that, while still asking for a convergence of $L_{l o c}^{1}$ type, we will possibly treat the first $N$ components of the spatial variable (representing the current state in a delay differential equation) in a different way from the last $N$ ones (representing the history of the state). Firstly, let us consider the set of Lipschitz Carathéodory functions $\mathfrak{L} \mathfrak{C}\left(\mathbb{R}^{2 N}, \mathbb{R}^{N}\right.$ ) (see Definition 2.1) and introduce the notion of $l_{1}$-bounds and $l_{2}$-bounds.

Definition $5.2\left(l_{1}\right.$-bounds and $l_{2}$-bounds in $\left.\mathfrak{C}\left(\mathbb{R}^{2 N}, \mathbb{R}^{N}\right)\right)$. Let us consider a function $f \in \mathfrak{C}\left(\mathbb{R}^{2 N}, \mathbb{R}^{N}\right)$. We say that $f$ admits $l_{1}$-bounds (resp. $l_{2}$-bounds) if for every $j \in \mathbb{N}$ there exists a function $l_{1}^{j}(\cdot) \in L_{l o c}^{1}\left(\operatorname{resp} . l_{2}^{j}(\cdot) \in L_{l o c}^{1}\right)$ such that for almost every $t \in \mathbb{R}$

$$
\left|f\left(t, x_{1}, u\right)-f\left(t, x_{2}, u\right)\right| \leq l_{1}^{j}(t)\left|x_{1}-x_{2}\right| \quad \text { for all }\left(x_{1}, u\right),\left(x_{2}, u\right) \in B_{j}
$$

(resp. $\left|f\left(t, x, u_{1}\right)-f\left(t, x, u_{2}\right)\right| \leq l_{2}^{j}(t)\left|u_{1}-u_{2}\right| \quad$ for all $\left.\left(x, u_{1}\right),\left(x, u_{2}\right) \in B_{j}\right)$.

In particular, if $f \in \mathfrak{L} \mathfrak{C}\left(\mathbb{R}^{2 N}, \mathbb{R}^{N}\right)$, i.e. $f: \mathbb{R} \times \mathbb{R}^{2 N} \rightarrow \mathbb{R}^{N}$ satisfying the assumptions in Definition 2.1, for every $j \in \mathbb{N}$ we refer to the optimal $l_{1}$-bound and the optimal $l_{2}$-bound for $f$ on $B_{j} \subset \mathbb{R}^{2 N}$ as to

$$
\begin{aligned}
& l_{1}^{j}(t)=\sup _{\substack{\left(x_{1}, u\right),\left(x_{2}, u\right) \in B_{j} \\
x_{1} \neq x_{2}}} \frac{\left|f\left(t, x_{1}, u\right)-f\left(t, x_{2}, u\right)\right|}{\left|x_{1}-x_{2}\right|}, \\
& l_{2}^{j}(t)=\sup _{\substack{\left(x, u_{1}\right),\left(x, u_{2}\right) \in B_{j} \\
u_{1} \neq u_{2}}} \frac{\left|f\left(t, x, u_{1}\right)-f\left(t, x, u_{2}\right)\right|}{\left|u_{1}-u_{2}\right|} .
\end{aligned}
$$

If $f \in \mathfrak{S} \mathfrak{C}\left(\mathbb{R}^{2 N}, \mathbb{R}^{N}\right)$ one can still define either the optimal $l_{1}$-bounds and/or the optimal $l_{2}$-bounds if, for almost every $t \in \mathbb{R}, f$ is Lipschitz continuous with respect to the first and/or the last $N$ space variables, respectively. 
Remark 5.3. Consider $f \in \mathfrak{L} \mathfrak{C}\left(\mathbb{R}^{2 N}, \mathbb{R}^{N}\right)$. We claim that for all $t \in \mathbb{R}$ one has $l^{j}(t) \leq l_{1}^{j}(t)+l_{2}^{j}(t)$, where by $l^{j}(\cdot)$ we denote the optimal $l$-bound for $f$ on $B_{j}$ as in (2.2). In order to prove it, firstly observe that if $\left(x_{1}, u_{1}\right),\left(x_{2}, u_{2}\right) \in B_{j} \subset \mathbb{R}^{2 N}$, then, at least one between $\left(x_{1}, u_{2}\right)$ and $\left(x_{2}, u_{1}\right)$ is in $B_{j} \subset \mathbb{R}^{2 N}$. Otherwise, we would have

$$
\left|\left(x_{1}, u_{2}\right)\right|^{2}>j^{2} \quad \text { and } \quad\left|\left(x_{2}, u_{1}\right)\right|^{2}>j^{2} .
$$

However, by assumption we know that

$$
\left|\left(x_{1}, u_{1}\right)\right|^{2} \leq j^{2} \quad \text { and } \quad\left|\left(x_{2}, u_{2}\right)\right|^{2} \leq j^{2},
$$

and thus we would obtain the following contradiction

$$
2 j^{2}<\left|\left(x_{1}, u_{2}\right)\right|^{2}+\left|\left(x_{2}, u_{1}\right)\right|^{2}=\left|\left(x_{1}, u_{1}\right)\right|^{2}+\left|\left(x_{2}, u_{2}\right)\right|^{2} \leq 2 j^{2} .
$$

Therefore, for any $t \in \mathbb{R}$, any pair $\left(x_{1}, u_{1}\right),\left(x_{2}, u_{2}\right) \in B_{j} \subset \mathbb{R}^{2 N}$, and assuming without any loss of generality that $\left(x_{2}, u_{1}\right) \in B_{j}$, one has

$$
\begin{aligned}
& \frac{\left|f\left(t, x_{1}, u_{1}\right)-f\left(t, x_{2}, u_{2}\right)\right|}{\left|\left(x_{1}, u_{1}\right)-\left(x_{2}, u_{2}\right)\right|} \\
& \leq \frac{\left|f\left(t, x_{1}, u_{1}\right)-f\left(t, x_{2}, u_{1}\right)\right|+\left|f\left(t, x_{2}, u_{1}\right)-f\left(t, x_{2}, u_{2}\right)\right|}{\left|\left(x_{1}, u_{1}\right)-\left(x_{2}, u_{2}\right)\right|} \\
& \leq l_{1}^{j}(t) \frac{\left|x_{1}-x_{2}\right|}{\left|\left(x_{1}, u_{1}\right)-\left(x_{2}, u_{2}\right)\right|}+l_{2}^{j}(t) \frac{\left|u_{1}-u_{2}\right|}{\left|\left(x_{1}, u_{1}\right)-\left(x_{2}, u_{2}\right)\right|} \leq l_{1}^{j}(t)+l_{2}^{j}(t),
\end{aligned}
$$

which gives us the aimed inequality once, in the previous formula, one takes the superior over $\left(x_{1}, u_{1}\right),\left(x_{2}, u_{2}\right) \in B_{j}$, with $\left(x_{1}, u_{1}\right) \neq\left(x_{2}, u_{2}\right)$.

As follows, we introduce some new topologies on $\mathfrak{S} \mathfrak{C}\left(\mathbb{R}^{2 N}, \mathbb{R}^{N}\right)$.

Definition 5.4 (Hybrid topologies on $\mathfrak{S C}\left(\mathbb{R}^{2 N}, \mathbb{R}^{N}\right)$ ). Let $\Theta$ and $\widehat{\Theta}$ be suitable sets of moduli of continuity as defined in Definition 2.3, $D$ be a countable dense subset of $\mathbb{R}^{N}$ and, for any $I=\left[q_{1}, q_{2}\right], q_{1}, q_{2} \in \mathbb{Q}$ and $j \in \mathbb{N}$, let $\mathcal{K}_{j}^{I}$ and $\widehat{\mathcal{K}}_{j}^{I}$ be the sets of functions in $C\left(I, B_{j}\right)$ which admit $\theta_{j}^{I}$ and $\hat{\theta}_{j}^{I}$, respectively, as a moduli of continuity.

- We call $\mathcal{T}_{\Theta D}$ the topology on $\mathfrak{S} \mathfrak{C}\left(\mathbb{R}^{2 N}, \mathbb{R}^{N}\right)$ generated by the family of seminorms

$$
p_{I, u, j}(f)=\sup _{x(\cdot) \in \mathcal{K}_{j}^{I}} \int_{I}|f(t, x(t), u)| d t, \quad f \in \mathfrak{S} \mathfrak{C}\left(\mathbb{R}^{2 N}, \mathbb{R}^{N}\right)
$$

with $I=\left[q_{1}, q_{2}\right], q_{1}, q_{2} \in \mathbb{Q}, u \in D$ and $j \in \mathbb{N}$. $\left(\mathfrak{S} \mathfrak{C}\left(\mathbb{R}^{2 N}, \mathbb{R}^{N}\right), \mathcal{T}_{\Theta D}\right)$ is a locally convex metric space. 
- We call $\mathcal{T}_{\Theta} \widehat{\Theta}$ the topology on $\mathfrak{S} \mathfrak{C}\left(\mathbb{R}^{2 N}, \mathbb{R}^{N}\right)$ generated by the family of seminorms

$$
p_{I, j}(f)=\sup _{x(\cdot) \in \mathcal{K}_{j}^{I}, u(\cdot) \in \widehat{\mathcal{K}}_{j}^{I-1}} \int_{I}|f(t, x(t), u(t-1))| d t, \quad f \in \mathfrak{S} \mathfrak{C}\left(\mathbb{R}^{2 N}, \mathbb{R}^{N}\right),
$$

with $I=\left[q_{1}, q_{2}\right], q_{1}, q_{2} \in \mathbb{Q}$ and $j \in \mathbb{N} .\left(\mathfrak{S C}\left(\mathbb{R}^{2 N}, \mathbb{R}^{N}\right), \mathcal{T}_{\Theta} \widehat{\Theta}\right)$ is a locally convex metric space.

- We call $\mathcal{T}_{\Theta B}$ the topology on $\mathfrak{S} \mathfrak{C}\left(\mathbb{R}^{2 N}, \mathbb{R}^{N}\right)$ generated by the family of seminorms

$$
p_{I, j}(f)=\sup _{x(\cdot) \in \mathcal{K}_{j}^{I}, u(\cdot) \in C\left(I-1, B_{j}\right)} \int_{I}|f(t, x(t), u(t-1))| d t, f \in \mathfrak{S} \mathfrak{C}\left(\mathbb{R}^{2 N}, \mathbb{R}^{N}\right),
$$

with $I=\left[q_{1}, q_{2}\right], q_{1}, q_{2} \in \mathbb{Q}$ and $j \in \mathbb{N} .\left(\mathfrak{S C}\left(\mathbb{R}^{2 N}, \mathbb{R}^{N}\right), \mathcal{T}_{\Theta B}\right)$ is a locally convex metric space.

- We call $\sigma_{\Theta D}$ the topology on $\mathfrak{S C}\left(\mathbb{R}^{2 N}, \mathbb{R}^{N}\right)$ generated by the family of seminorms

$$
p_{I, u, j}(f)=\sup _{x(\cdot) \in \mathcal{K}_{j}^{I}}\left|\int_{I}[f(t, x(t), u)] d t\right|, \quad f \in \mathfrak{S} \mathfrak{C}\left(\mathbb{R}^{2 N}, \mathbb{R}^{N}\right),
$$

with $I=\left[q_{1}, q_{2}\right], q_{1}, q_{2} \in \mathbb{Q}, u \in D$ and $j \in \mathbb{N}$. $\left(\mathfrak{S} \mathfrak{C}\left(\mathbb{R}^{2 N}, \mathbb{R}^{N}\right), \sigma_{\Theta D}\right)$ is a locally convex metric space.

- We call $\sigma_{\Theta \Theta}$ the topology on $\mathfrak{S} \mathfrak{C}\left(\mathbb{R}^{2 N}, \mathbb{R}^{N}\right)$ generated by the family of seminorms

$$
p_{I, j}(f)=\sup _{x(\cdot) \in \mathcal{K}_{j}^{I}, u(\cdot) \in \widehat{\mathcal{K}}_{j}^{I-1}}\left|\int_{I}[f(t, x(t), u(t-1))] d t\right|, \quad f \in \mathfrak{S} \mathfrak{C}\left(\mathbb{R}^{2 N}, \mathbb{R}^{N}\right),
$$

with $I=\left[q_{1}, q_{2}\right], q_{1}, q_{2} \in \mathbb{Q}$ and $j \in \mathbb{N} .\left(\mathfrak{S C}\left(\mathbb{R}^{2 N}, \mathbb{R}^{N}\right), \sigma_{\Theta} \widehat{\Theta}\right)$ is a locally convex metric space.

The topologies introduced in the previous definition are called hybrid because they are derived from the ones presented in Chapter 2 so that the condition of uniformity for the first $N$ space variables differ from the one of the remaining $N$ space variables. A natural question arises, concerning the relation between the topologies $\mathcal{T}_{\Theta}$ and $\mathcal{T}_{\Theta \Theta}$ (resp. $\sigma_{\Theta}$ and $\left.\sigma_{\Theta \Theta}\right)$ on $\mathfrak{S} \mathfrak{C}\left(\mathbb{R}^{2 N}, \mathbb{R}^{N}\right)$. In order to deal with such a problem, we need to state the following technical lemma for the topologies of the type $\sigma_{\Theta} \widehat{\Theta}$ (which is the analogous of Lemma 2.14 for $\sigma_{\Theta}$ ). Since its proof differs from the one of Lemma 2.14 only on minor details, we skip it. 
Lemma 5.5. Let $\Theta$ and $\widehat{\Theta}$ be suitable sets of moduli of continuity as in Definition 2.3 and, for each $j \in \mathbb{N}$ and $I=\left[q_{1}, q_{2}\right], q_{1}, q_{2} \in \mathbb{Q}$, let $\mathcal{K}_{j}^{I}$ and $\widehat{\mathcal{K}}_{j}^{I}$ be the compact sets in $C\left(I, B_{j}\right)$ which admit $\theta_{j}^{I}$ and $\hat{\theta}_{j}^{I}$, respectively, as a modulus of continuity.

(i) Let $f$ be a function of $\mathfrak{S} \mathfrak{C}\left(\mathbb{R}^{2 N}, \mathbb{R}^{N}\right)$. For each $j \in \mathbb{N}$ and $I=\left[q_{1}, q_{2}\right]$, $q_{1}, q_{2} \in \mathbb{Q}$, if $\left(x_{n}(\cdot)\right)_{n \in \mathbb{N}}$ is a sequence in $\mathcal{K}_{j}^{I}$ converging uniformly to some function $x(\cdot) \in \mathcal{K}_{j}^{I}$ and $\left(u_{n}(\cdot)\right)_{n \in \mathbb{N}}$ is a sequence in $\widehat{\mathcal{K}}_{j}^{I-1}$ converging uniformly to some function $u(\cdot) \in \widehat{\mathcal{K}}_{j}^{I-1}$, then

$$
\lim _{n \rightarrow \infty} \int_{p_{1}}^{p_{2}} f\left(t, x_{n}(t), u_{n}(t-1)\right) d t=\int_{p_{1}}^{p_{2}} f(t, x(t), u(t-1)) d t
$$

whenever $p_{1}, p_{2} \in \mathbb{Q}$ and $q_{1} \leq p_{1}<p_{2} \leq q_{2}$.

(ii) Let $\left(g_{n}\right)_{n \in \mathbb{N}}$ be a sequence in $\mathfrak{S} \mathfrak{C}\left(\mathbb{R}^{2 N}, \mathbb{R}^{N}\right)$ converging to some function $g$ in $\left(\mathfrak{S C}\left(\mathbb{R}^{N}\right), \sigma_{\Theta} \widehat{\Theta}\right)$. Then, for any $I=\left[q_{1}, q_{2}\right], q_{1}, q_{2} \in \mathbb{Q}$ and $j \in \mathbb{N}$ one has

$$
\lim _{n \rightarrow \infty} \sup _{x(\cdot) \in \mathcal{K}_{j}^{I}, u(\cdot) \in \widehat{\mathcal{K}}_{j}^{I-1}}\left|\int_{p_{1}}^{p_{2}}\left[g_{n}(t, x(t), u(t-1))-g(t, x(t), u(t-1))\right] d t\right|=0,
$$

whenever $p_{1}, p_{2} \in \mathbb{Q}$ and $q_{1} \leq p_{1}<p_{2} \leq q_{2}$.

Then, we are finally ready to relate the topologies $\mathcal{T}_{\Theta}$ and $\mathcal{T}_{\Theta \Theta}$ (resp. $\sigma_{\Theta}$ and $\left.\sigma_{\Theta \Theta}\right)$ on $\mathfrak{S C}$.

Proposition 5.6. Let $\Theta$ be a suitable set of moduli of continuity as in Definition 2.3. Considered the topologies $\mathcal{T}_{\Theta}, \sigma_{\Theta}, \mathcal{T}_{\Theta \Theta}$ and $\sigma_{\Theta \Theta}$ introduced in Definitions 2.9, 2.10, and 5.4, respectively, the following order relations hold in $\mathfrak{S} \mathfrak{C}$ :

$$
\mathcal{T}_{\Theta} \leq \mathcal{T}_{\Theta \Theta} \quad \text { and } \quad \sigma_{\Theta} \leq \sigma_{\Theta \Theta}
$$

Proof. We will complete the proof for the weak topologies because the other one is analogous (and simpler). Furthermore, in order to avoid any abuse of notation, within this proof we will write

$$
\begin{aligned}
\mathcal{K}_{j, 2 N}^{I} & :=\left\{\xi: I \rightarrow B_{j} \subset \mathbb{R}^{2 N}|| \xi(t)-\xi(s) \mid \leq \theta_{j}^{I}(|t-s|), \text { for all } t, s \in I\right\}, \\
\mathcal{K}_{j, N}^{I} & :=\left\{\eta: I \rightarrow B_{j} \subset \mathbb{R}^{N}|| \eta(t)-\eta(s) \mid \leq \theta_{j}^{I}(|t-s|), \text { for all } t, s \in I\right\} .
\end{aligned}
$$

Firstly, let us consider a sequence $\left(f_{n}\right)_{n \in \mathbb{N}}$ in $\mathfrak{S} \mathfrak{C}$ converging to some $f \in \mathfrak{S} \mathfrak{C}$ with respect to the topology $\sigma_{\Theta \Theta}$ and prove that one also has $f_{n} \stackrel{\sigma_{\Theta}}{\longrightarrow} f$ as $n \rightarrow \infty$. To the aim, fix $I=\left[q_{1}, q_{2}\right], q_{1}, q_{2} \in \mathbb{Q}$, and $j \in \mathbb{N}$ and consider $J=\left[p_{1}, p_{2}\right]$, 
$p_{1}, p_{2} \in \mathbb{Q}$ such that $I \subset J$ and $I \cup(I-1) \subset J-1$. Then, one has

$$
\begin{aligned}
& \sup _{(x(\cdot), u(\cdot)) \in \mathcal{K}_{j, 2 N}^{I}}\left|\int_{I}\left[f_{n}(t, x(t), u(t))-f(t, x(t), u(t))\right] d t\right| \\
& \quad \leq \sup _{x(\cdot) \in \mathcal{K}_{j, N}^{I}, u(\cdot) \in \mathcal{K}_{j, N}^{I}}\left|\int_{I}\left[f_{n}(t, x(t), u(t))-f(t, x(t), u(t))\right] d t\right| \\
& \quad \leq \sup _{x(\cdot) \in \mathcal{K}_{j, N}^{J}, u(\cdot) \in \mathcal{K}_{j, N}^{J-1}}\left|\int_{I}\left[f_{n}(t, x(t), u(t-1))-f(t, x(t), u(t-1))\right] d t\right|
\end{aligned}
$$

where the last inequality is obtained up to extending by constants to $J$ the functions $x(\cdot) \in \mathcal{K}_{j, N}^{I}$, and up to changing any function $u(\cdot) \in \mathcal{K}_{j, N}^{I}$ for its translation $\tilde{u}: I-1 \rightarrow B_{j} \subset \mathbb{R}^{N}$ so that $\tilde{u}(t)=u(t+1)$ for all $t \in I-1$, and up to extending such functions $\widetilde{u}$ by constant to $J-1$. Now, by assumption we have that

$$
\sup _{x(\cdot) \in \mathcal{K}_{j, N}^{J}, u(\cdot) \in \mathcal{K}_{j, N}^{J-1}}\left|\int_{J}\left[f_{n}(t, x(t), u(t-1))-f(t, x(t), u(t-1))\right] d t\right| \stackrel{n \rightarrow \infty}{\longrightarrow} 0,
$$

and thus, passing to the limit as $n \rightarrow \infty$ in (5.3), we obtain the result thanks to Lemma 5.5(ii) and recalling that $I \subset J$.

Remark 5.7. Consider any dense and countable set $D \subset \mathbb{R}^{N}$, and any pair $\Theta$ and $\widehat{\Theta}$ of suitable sets of moduli of continuity as in Definition 2.3 such that, for any $I=\left[q_{1}, q_{2}\right], q_{1}, q_{2} \in \mathbb{Q}$ and $j \in \mathbb{N}$, one has

$$
\theta_{j}^{I}(t) \leq \hat{\theta}_{j}^{I}(t), \quad \text { for all } t \in[0, \infty) .
$$

Then, the following chains of order relate the topologies introduced in Definition 5.4 to some of the topologies presented in Section 2.2 when all of them are considered on $\mathfrak{S} \mathfrak{C}\left(\mathbb{R}^{2 N}, \mathbb{R}^{N}\right)$ :

$$
\begin{gathered}
\sigma_{D} \leq \mathcal{T}_{D} \leq \mathcal{T}_{\Theta D} \leq \mathcal{T}_{\Theta} \leq \mathcal{T}_{\Theta \Theta} \leq \mathcal{T}_{\Theta \widehat{\Theta}} \leq \mathcal{T}_{\Theta B} \leq \mathcal{T}_{B} \quad \text { and } \\
\sigma_{D} \leq \sigma_{\Theta D} \leq \sigma_{\Theta} \leq \sigma_{\Theta \Theta} \leq \sigma_{\Theta \widehat{\Theta}} \leq \mathcal{T}_{\Theta \widehat{\Theta}}
\end{gathered}
$$

where, in particular, the order relations $\mathcal{T}_{\Theta} \leq \mathcal{T}_{\Theta \Theta}$ and $\sigma_{\Theta} \leq \sigma_{\Theta \Theta}$ hold true thanks to Proposition 5.6. As a consequence, we have that some of the theorems proved in Chapter 2 already give information about the new topologies introduced in Definition 5.4. In particular, the results of propagation of the $L_{l o c}^{1}$-boundedness and/or $L_{l o c}^{1}$-equicontinuity of the $m$-bounds and/or $l$-bounds from a set to its topological closure contained in Propositions 2.26, 2.27 and 2.29 directly apply. Furthermore, by checking the proof of Theorem 2.31, one immediately notice that all the strong topologies in the first chain of (5.4) coincide on the subsets of $\mathfrak{L} \mathfrak{C}\left(\mathbb{R}^{2 N}, \mathbb{R}^{N}\right)$ with $L_{\text {loc }}^{1}$-bounded l-bounds. Consequently, analogous comments hold for the results of relative compactness contained in Section 2.6.

In analogy of Proposition 2.26, we present a result of propagation of the $l_{2}$-bounds with respect to all the considered topologies. 
Proposition 5.8. Let $\mathcal{T}$ be any of the topologies depicted in (5.4), and whose definitions are contained either in Section 2.2 or in Definition 5.4. The following statements hold true.

(i) If $E \subset \mathfrak{S C}\left(\mathbb{R}^{2 N}, \mathbb{R}^{N}\right)$ has $L_{\text {loc }}^{1}$-bounded $l_{2}$-bounds then $\operatorname{cls}_{\left(\mathfrak{S C}\left(\mathbb{R}^{2 N}\right), \mathcal{T}\right)}(E)$ has $L_{\text {loc }}^{1}$-bounded $l_{2}$-bounds.

(ii) If $f \in \mathfrak{S C}\left(\mathbb{R}^{2 N}, \mathbb{R}^{N}\right)$ admits $L_{\text {loc }}^{1}$-bounded $l_{2}$-bounds then also any $g \in$ $\operatorname{Hull}_{\left(\mathfrak{S C}\left(\mathbb{R}^{2 N}\right), \mathcal{T}\right)}(f)$ has $L_{\text {loc }}^{1}$-bounded $l_{2}$-bounds.

Proof. Let $D$ be any countable dense subset of $\mathbb{R}^{2 N}$. By (5.4), if we prove the result for the topology $\sigma_{D}$, then we have it for all the other topologies. Furthermore, in order to simplify the notation, let

$$
\bar{E}=\operatorname{cls}_{\left(\mathfrak{S C}\left(\mathbb{R}^{N}\right), \sigma_{D}\right)}(E) .
$$

Consider $f \in \bar{E}$ and let $\left(f_{n}\right)_{n \in \mathbb{N}}$ be a sequence in $E$ converging to $f$ with respect to $\sigma_{D}$. For any $j, n \in \mathbb{N}$ let us denote by $l_{n}^{j}(\cdot) \in L_{\text {loc }}^{1}$ the optimal $l_{2}$-bound of $f_{n}$ on $B_{j}$. Consider $t, h \in \mathbb{Q}$, with $h>0$ and $\left(x, u_{1}\right),\left(x, u_{2}\right) \in B_{j} \cap D$. Then one has

$$
\begin{aligned}
& \left|\int_{t}^{t+h}\left[f\left(s, x, u_{1}\right)-f\left(s, x, u_{2}\right)\right] d s\right| \leq\left|\int_{t}^{t+h}\left[f\left(s, x, u_{1}\right)-f_{n}\left(s, x, u_{1}\right)\right] d s\right|+ \\
& \quad+\left|\int_{t}^{t+h}\left[f_{n}\left(s, x, u_{1}\right)-f_{n}\left(s, x, u_{2}\right)\right] d s\right|+\left|\int_{t}^{t+h}\left[f_{n}\left(s, x, u_{2}\right)-f\left(s, x, u_{2}\right)\right] d s\right| .
\end{aligned}
$$

Notice that, since $\left(f_{n}\right)_{n \in \mathbb{N}}$ converges to $f$ in $\left(\mathfrak{S C}\left(\mathbb{R}^{2 N}, \mathbb{R}^{N}\right), \sigma_{D}\right)$, then the first and the last integrals on the right-hand side of the previous inequality tend to zero as $n \rightarrow \infty$. Moreover, one has

$$
\frac{1}{h}\left|\int_{t}^{t+h}\left[f_{n}\left(s, x, u_{1}\right)-f_{n}\left(s, x, u_{2}\right)\right] d s\right| \leq \frac{\left|u_{1}-u_{2}\right|}{h} \int_{t}^{t+h} l_{n}^{j}(s) d s,
$$

and by the $L_{l o c}^{1}$-boundedness of the $l_{2}$-bounds we have that the set of positive measures $\left\{\lambda_{n}^{j} \mid n \in \mathbb{N}\right\}$ with densities $\left\{l_{n}^{j}(\cdot) \mid n \in \mathbb{N}\right\}$ is relatively compact in $\mathcal{M}^{+}$with respect to the vague topology (see Remark 2.21 and Definition 2.19). Therefore, there exists $\lambda^{j} \in \mathcal{M}^{+}$such that, up to a subsequence, one has $\lambda_{n}^{j} \stackrel{\widetilde{\sigma}}{\rightarrow} \lambda^{j}$. Moreover, recall that by the regularity of $\lambda^{j}$, one has

$$
\lambda^{j}([t, t+h])=\inf \left\{\int_{\mathbb{R}} \phi(s) d \lambda^{j}(s) \mid \phi \in C_{C}^{+}(\mathbb{R}), \phi \equiv 1 \text { in }[t, t+h]\right\} .
$$

Hence, dividing both the sides in (5.5) by $h$, taking the limit as $n \rightarrow \infty$ and gathering all the previous reasoning, one has

$$
\frac{1}{h}\left|\int_{t}^{t+h}\left[f\left(s, x, u_{1}\right)-f\left(s, x, u_{2}\right)\right] d s\right| \leq \frac{\lambda^{j}([t, t+h])}{h}\left|u_{1}-u_{2}\right| .
$$


Now, consider $t, h \in \mathbb{R}$, with $h>0$, and let $\left(s_{n}\right)_{n \in \mathbb{N}}$ and $\left(t_{n}\right)_{n \in \mathbb{N}}$ be two sequences in $\mathbb{Q}$ such that, as $n \rightarrow \infty, s_{n} \downarrow t$ and $t_{n} \uparrow t+h$, respectively. By (5.6), applied on the intervals $\left[s_{n}, t_{n}\right]$, and noticing that $\lambda^{j}\left(\left[s_{n}, t_{n}\right)\right] \leq \lambda^{j}([t, t+h])$ for every $n \in \mathbb{N}$, one can write

$$
\frac{1}{h}\left|\int_{s_{n}}^{t_{n}}\left[f\left(s, x, u_{1}\right)-f\left(s, x, u_{2}\right)\right] d s\right| \leq \frac{\lambda^{j}([t, t+h])}{h}\left|u_{1}-u_{2}\right| .
$$

Hence, passing to the limit as $n \rightarrow \infty$ and using the continuity of the integral, one obtains (5.6) for every $t, h \in \mathbb{R}$ with $h>0$.

Now, by the Lebesgue-Besicovitch differentiation theorem (see [1, Theorem 2.22, p.54]), there exists $l^{j}(\cdot) \in L_{l o c}^{1}$ such that

$$
\lim _{h \rightarrow 0} \frac{\lambda^{j}([t, t+h])}{h}=l^{j}(t) \quad \text { for a.e. } t \in \mathbb{R}
$$

and $l^{j}(\cdot)$ is the density of the absolutely continuous part of the Radon-Nikodym decomposition of $\lambda^{j}$. Therefore, as $h \rightarrow 0$ (see [24, Corollary III.12.7, p.216]), (5.6) becomes

$$
\left|f\left(t, x, u_{1}\right)-f\left(t, x, u_{2}\right)\right| \leq l^{j}(t)\left|u_{1}-u_{2}\right| \quad \text { for a.e. } t \in \mathbb{R} \text {. }
$$

The set $R\left(x, u_{1}, u_{2}\right) \subset \mathbb{R}$ such that $\operatorname{meas}\left(\mathbb{R} \backslash R\left(x, u_{1}, u_{2}\right)\right)=0$ where (5.8) holds, depends on $x, u_{1}$ and $u_{2}$. However, since $D$ is numerable, by simply intersecting all the possible $R\left(x, u_{1}, u_{2}\right)$, with $\left(x, u_{1}\right),\left(x, u_{2}\right) \in B_{j} \cap D$ (there is only a numerable quantity of them), one obtains a set $R_{0} \subset \mathbb{R}$ of full measure for which (5.8) holds for all $\left(x, u_{1}\right),\left(x, u_{2}\right) \in B_{j} \cap D$. Nevertheless, (5.8) can be extended to all $\left(x, u_{1}\right),\left(x, u_{2}\right) \in B_{j}$ through the continuity of $f$ with respect to the last variables; which proves that the functions in $\bar{E}$ admit a Lipschitz coefficient in the third variable.

Finally, we prove that $\bar{E}$ admits $L_{\text {loc }}^{1}$-bounded $l_{2}$-bounds. For each $f \in \bar{E}$ and any $j \in \mathbb{N}$, let $l_{f}^{j}$ be either, the optimal $l_{2}$-bound of $f$ on $B^{j}$ if $f \in E$, or the $l_{2}$-bound given by (5.7) if $f \in \bar{E} \backslash E$, i.e. the absolutely continuous part of a limit measure. Moreover, for each $f \in \bar{E}$, let $\left(f_{n}\right)_{n \in \mathbb{N}}$ be a sequence in $E$ converging to $f$ with respect to $\sigma_{D}$. Consider $j \in \mathbb{N}, r>0$ and $\phi \in C_{C}^{+}$such that $\operatorname{supp} \phi \subset[-r-1, r+1]$ and $\phi \equiv 1$ in $[-r, r]$, then, we have

$$
\begin{aligned}
\int_{-r}^{r} l_{f}^{j}(t) d t & \leq \int_{\mathbb{R}} \phi(t) l_{f}^{j}(t) d t \leq \lim _{n \rightarrow \infty} \int_{\mathbb{R}} \phi(t) l_{f_{n}}^{j}(t) d t \\
& \leq \sup _{g \in E} \int_{-r-1}^{r+1} l_{g}^{j}(t) d t<\infty
\end{aligned}
$$

which ends the proof.

Considered the different role played in delay differential equations by the first $N$ and the last $N$ space variables of the vector field, and the notion of $l_{1}$ and $l_{2}$ 
bounds given in Definition 5.2, at this point we would like to see if it is possible to deduce a result similar to Theorem 2.33, but just for the hybrid topologies presented in Definition 5.4.

Theorem 5.9. Let $E$ be a set in $\mathfrak{S} \mathfrak{C}\left(\mathbb{R}^{2 N}, \mathbb{R}^{N}\right)$ with $L_{\text {loc }}^{1}$-bounded $l_{2}$-bounds. If $D$ is any dense and numerable subset of $\mathbb{R}^{N}$, and $\Theta$ and $\widehat{\Theta}$ are any pair of suitable sets of moduli of continuity (see Definition 2.3), then

$$
\left(E, \mathcal{T}_{1}\right)=\left(E, \mathcal{T}_{2}\right) \quad \text { and } \quad \operatorname{cls}_{\left(\mathfrak{S C}\left(\mathbb{R}^{2 N}, \mathbb{R}^{N}\right), \mathcal{T}_{1}\right)}(E)=\operatorname{cls}_{\left(\mathfrak{S C}\left(\mathbb{R}^{2 N}, \mathbb{R}^{N}\right), \mathcal{T}_{2}\right)}(E) .
$$

where $\mathcal{T}_{1}$ and $\mathcal{T}_{2}$ are any of the strong topologies introduced in Definition 5.4. Furthermore, one also has that

$$
\left(E, \sigma_{\Theta D}\right)=\left(E, \sigma_{\Theta \widehat{\Theta}}\right) \quad \text { and } \quad \operatorname{cls}_{\left(\mathfrak{S} \mathfrak{C}\left(\mathbb{R}^{2 N}, \mathbb{R}^{N}\right), \sigma_{\Theta D}\right)}(E)=\operatorname{cls}_{\left(\mathfrak{S C}\left(\mathbb{R}^{2 N}, \mathbb{R}^{N}\right), \sigma_{\Theta} \widehat{\Theta}\right)}(E) .
$$

Proof. We will prove the case of the weak topologies and omit the proof for the strong topologies because it is analogous and simpler. Moreover, in order to simplify the notation, let us use the following symbols:

$$
\bar{E}_{\Theta D}=\operatorname{cls}_{\left(\mathfrak{S C}\left(\mathbb{R}^{2 N}, \mathbb{R}^{N}\right), \sigma_{\Theta D}\right)}(E) \quad \text { and } \quad \bar{E}_{\Theta \widehat{\Theta}}=\operatorname{cls}_{\left(\mathfrak{S}\left(\mathbb{R}^{2 N}, \mathbb{R}^{N}\right), \sigma_{\Theta}\right)}(E) .
$$

Thanks to Proposition 5.8, we know that $\bar{E}_{\Theta D}$ has $L_{l o c}^{1}$-bounded $l_{2}$-bounds. In analogy with Theorem 2.33, we will complete the proof in two steps.

Step 1. Consider a set $E_{1}$ in $\mathfrak{S C}\left(\mathbb{R}^{2 N}, \mathbb{R}^{N}\right)$ with $L_{\text {loc }}^{1}$-bounded $m$-bounds and $L_{l o c}^{1}$-bounded $l_{2}$-bounds. Let $\left(f_{n}\right)_{n \in \mathbb{N}}$ be a sequence of elements of $E_{1}$ converging to some $f$ in $\left(\mathfrak{S C}\left(\mathbb{R}^{2 N}, \mathbb{R}^{N}\right), \sigma_{\Theta D}\right)$. We shall prove that $\left(f_{n}\right)_{n \in \mathbb{N}}$ converges to $f$ in $\left(\mathfrak{S C}\left(\mathbb{R}^{2 N}, \mathbb{R}^{N}\right), \sigma_{\Theta} \widehat{\Theta}\right)$ where $\Theta$ and $\widehat{\Theta}$ are suitable sets of moduli of continuity. Fix a compact interval $I=\left[q_{1}, q_{2}\right]$, with $q_{1}, q_{2} \in \mathbb{Q}, j \in \mathbb{N}$ and, for any $n \in \mathbb{N}$, let $m_{n}^{2 j}(\cdot) \in L_{l o c}^{1}$ and $l_{n}^{2 j}(\cdot) \in L_{l o c}^{1}$ be respectively the optimal $m$-bound and the optimal $l_{2}$-bound of $f_{n}$ on $B_{2 j} \subset \mathbb{R}^{2 N}$. By the $L_{l o c}^{1}$-boundedness of the $l_{2}$-bounds, there exists $\rho>0$ such that

$$
\sup _{n \in \mathbb{N}} \int_{I} l_{n}^{2 j}(s) d s<\rho<\infty .
$$

Fix $\varepsilon>0$ and consider $\delta=\varepsilon / 3 \rho$. Since $B_{j} \subset \mathbb{R}^{N}$ is compact, and $D$ is dense in $\mathbb{R}^{N}$, there exist $u_{1}, \ldots u_{\nu} \in D$ such that

$$
B_{j} \subset \bigcup_{i=1}^{\nu} B_{\delta}\left(u_{i}\right)
$$

where $B_{\delta}(u)$ denotes the closed ball of $\mathbb{R}^{N}$ of radius $\delta$ centered at $u \in \mathbb{R}^{N}$. For $i=1, \ldots, \nu$, let us consider the continuous functions $\phi_{i}: \mathbb{R}^{N} \rightarrow[0,1]$, so that

$$
\operatorname{supp}\left(\phi_{i}\right) \subset B_{\delta}\left(u_{i}\right) \quad \text { and } \quad \sum_{i=1}^{\nu} \phi_{i}(u)=1 \quad \forall u \in B_{j},
$$


and define the functions

$$
f_{n}^{*}(t, x, u)=\sum_{i=1}^{\nu} \phi_{i}(u) f_{n}\left(t, x, u_{i}\right) \quad \text { and } \quad f^{*}(t, x, u)=\sum_{i=1}^{\nu} \phi_{i}(u) f\left(t, x, u_{i}\right) .
$$

Denote by $\mathcal{K}_{j}^{I}$ and $\widehat{\mathcal{K}}_{j}^{I-1}$ the compact subsets of $C\left(I, B_{j}\right)$ and $C\left(I-1, B_{j}\right)$, with $B_{j} \subset \mathbb{R}^{N}$, admitting $\theta_{j}^{I} \in \Theta$ and $\hat{\theta}_{j}^{I-1} \in \widehat{\Theta}$, respectively, as moduli of continuity. For any $x(\cdot) \in \mathcal{K}_{j}^{I}$ and $u(\cdot) \in \widehat{\mathcal{K}}_{j}^{I-1}$ one has

$$
\begin{aligned}
\left|\int_{I}\left[f_{n}(t, x(t), u(t-1))-f(t, x(t), u(t-1))\right] d t\right| \\
\leq\left|\int_{I}\left[f_{n}(t, x(t), u(t-1))-f_{n}^{*}(t, x(t), u(t-1))\right] d t\right| \\
+\left|\int_{I}\left[f_{n}^{*}(t, x(t), u(t-1))-f^{*}(t, x(t), u(t-1))\right] d t\right| \\
+\left|\int_{I}\left[f^{*}(t, x(t), u(t-1))-f(t, x(t), u(t-1))\right] d t\right| .
\end{aligned}
$$

Let us separately analyze each element in the sum on the right-hand side of the previous inequality. As regards the first one, we have that

$$
\begin{aligned}
\left|\int_{I}\left[f_{n}(t, x(t), u(t-1))-f_{n}^{*}(t, x(t), u(t-1))\right] d t\right| \\
\quad=\left|\int_{I} \sum_{i=1}^{\nu} \phi_{i}(u(t-1))\left[f_{n}(t, x(t), u(t-1))-f_{n}\left(t, x(t), u_{i}\right)\right] d t\right| \\
\quad \leq \int_{I} \sum_{i=1}^{\nu} \phi_{i}(u(t-1)) l_{n}^{2 j}(t)\left|u(t-1)-u_{i}\right| d t \\
\leq \int_{I} \sum_{i=1}^{\nu} \phi_{i}(u(t-1)) l_{n}^{2 j}(t) \delta d t=\frac{\varepsilon}{3 \rho} \int_{I} l_{n}^{2 j}(t) d t \leq \frac{\varepsilon}{3},
\end{aligned}
$$

where the first inequality holds thanks to Definition 5.2 and recalling that for any $t \in I$, one has that $|(x(t), u(t-1))| \leq 2 j$. Similar reasonings apply to the third element of the sum in (5.9): in particular, recall that, due to Proposition 5.8, the $l_{2}$-bound for $f$ on $B_{2 j}$, namely $l^{2 j}(\cdot) \in L_{l o c}^{1}$, satisfies

$$
\int_{I} l^{2 j}(s) d s<\rho<\infty
$$

Finally, let us deal with the the remaining integral in (5.9). By the uniform continuity of the functions $\phi_{i}(\cdot)$ on $B_{j} \subset \mathbb{R}^{N}$, and recalling that all $u(\cdot) \in \widehat{\mathcal{K}}_{j}^{I-1}$ share the same modulus of continuity, we have that for the given $\varepsilon>0$ there exists $\delta>0$ such that for all $i \in\{1, \ldots, \nu\}$ one has

$$
\forall s, t \in I-1, \forall u(\cdot) \in \widehat{\mathcal{K}}_{j}^{I-1}: \quad|s-t|<\delta \Rightarrow\left|\phi_{i}(u(s))-\phi_{i}(u(t))\right|<\frac{\varepsilon}{9 \nu \rho_{m}},
$$


where

$$
\rho_{m}:=\max \left\{\int_{I} m^{2 j}(t) d t, \sup _{n \in \mathbb{N}} \int_{I} m_{n}^{2 j}(t) d t\right\}<\infty,
$$

and by $m_{2 j}(\cdot) \in L_{\text {loc }}^{1}$ we denote the optimal $m$-bound for $f$ on $B^{2 j} \subset \mathbb{R}^{2 N}$, whose existence is guaranteed by Proposition 2.26 and (5.4). In particular, the constant $\rho_{m}$ is well defined thanks to the $L_{l o c}^{1}$-boundedness of the $m$-bounds of the functions in $E_{1}$. Thus, let us consider a $\delta$-partition of $I$, i.e. $\tau_{1}, \ldots, \tau_{\eta} \in I \cap \mathbb{Q}$ such that $I=\left[\tau_{1}, \tau_{\eta}\right]$ and $0<\tau_{k+1}-\tau_{k}<\delta$, with $k=1, \ldots, \eta-1$, and a function

$\bar{\phi}_{i}: \widehat{\mathcal{K}}_{j}^{I-1} \rightarrow L^{\infty}(I, \mathbb{R}) \quad$ defined by $\quad \bar{\phi}_{i}(u(t-1))=\sum_{k=1}^{\eta} \phi_{i}\left(u\left(\tau_{k}-1\right)\right) \chi_{\left(\tau_{k}, \tau_{k+1}\right]}(t)$

Notice that, for any $u(\cdot) \in \widehat{\mathcal{K}}_{j}^{I-1}$ and any $i=1, \ldots, \nu$ one has

$$
\left\|\phi_{i}(u(\cdot-1))-\bar{\phi}_{i}(u(\cdot-1))\right\|_{L^{\infty}(I)}<\frac{\varepsilon}{9 \nu \rho_{m}} .
$$

Now, we have

$$
\begin{aligned}
& \left|\int_{I}\left[f_{n}^{*}(t, x(t), u(t-1))-f^{*}(t, x(t), u(t-1))\right] d t\right| \\
& \leq \sum_{i=1}^{\nu}\left|\int_{I} \phi_{i}(u(t-1))\left[f_{n}\left(t, x(t), u_{i}\right)-f\left(t, x(t), u_{i}\right)\right] d t\right| \\
& \leq \sum_{i=1}^{\nu}\left|\int_{I} \bar{\phi}_{i}(u(t-1))\left[f_{n}\left(t, x(t), u_{i}\right)-f\left(t, x(t), u_{i}\right)\right] d t\right|+ \\
& \quad+\sum_{i=1}^{\nu} \int_{I}\left|f_{n}\left(t, x(t), u_{i}\right)\right|\left|\phi_{i}(u(t-1))-\bar{\phi}_{i}(u(t-1))\right| d t+ \\
& \quad+\sum_{i=1}^{\nu} \int_{I}\left|f\left(t, x(t), u_{i}\right)\right|\left|\bar{\phi}_{i}(u(t-1))-\phi_{i}(u(t-1))\right| d t \\
& \leq \sum_{i=1}^{\nu}\left[\sum_{k=1}^{\eta} \phi_{i}\left(u\left(\tau_{k}-1\right)\right)\left|\int_{\tau_{k}}^{\tau_{k+1}}\left[f_{n}\left(t, x(t), u_{i}\right)-f\left(t, x(t), u_{i}\right)\right] d t\right|+\right. \\
& \left.\quad+2 \rho_{m}\left\|\phi_{i}(u(\cdot-1))-\bar{\phi}_{i}(u(\cdot-1))\right\|_{L^{\infty}(I)}\right] .
\end{aligned}
$$

By the convergence of $\left(f_{n}\right)_{n \in \mathbb{N}}$ to $f$ in $\left(\mathfrak{S C}\left(\mathbb{R}^{2 N}, \mathbb{R}^{N}\right), \sigma_{\Theta D}\right)$ and considering that we are only using a finite number of points $u_{i}$, with $i=1, \ldots, \nu$, there exists $n_{0} \in \mathbb{N}$ such that, if $n>n_{0}$, then for all $i=1, \ldots, \nu$ and for all $k=1, \ldots, \eta$ one has

$$
\left|\int_{\tau_{k}}^{\tau_{k+1}}\left[f_{n}\left(t, x(t), u_{i}\right)-f\left(t, x(t), u_{i}\right)\right] d t\right|<\frac{\varepsilon}{9 \nu \eta} .
$$

Thus for $n>n_{0}$, (5.11) becomes

$$
\left|\int_{I}\left[f_{n}^{*}(t, x(t), u(t-1))-f^{*}(t, x(t), u(t-1))\right] d t\right|<\frac{\varepsilon}{9}+\frac{2 \nu \rho_{m} \varepsilon}{9 \nu \rho_{m}}=\frac{\varepsilon}{3} .
$$


From (5.9), (5.10) and (5.12) we obtain that the sequence $\left(f_{n}\right)_{n \in \mathbb{N}}$ converges to $f$ in $\left(\mathfrak{S C}\left(\mathbb{R}^{2 N}, \mathbb{R}^{N}\right), \sigma_{\Theta} \widehat{\Theta}\right)$, and thus the equivalence of the topologies of type $\sigma_{\Theta D}$ and $\sigma_{\Theta} \widehat{\Theta}$ on $E_{1}$.

Step 2. Now, fixed a dense and countable set $D \subset \mathbb{R}^{N}$ and a pair $\Theta$ and $\widehat{\Theta}$ of suitable sets of moduli of conitnuity, assume that $\left(f_{n}\right)_{n \in \mathbb{N}}$ is a sequence of elements of $E$ converging to some $f$ in $\left(\mathfrak{S C}\left(\mathbb{R}^{2 N}, \mathbb{R}^{N}\right), \sigma_{\Theta D}\right)$. In order to complete the proof, we prove that $\left(f_{n}\right)_{n \in \mathbb{N}}$ converges to $f$ in $\left(\mathfrak{S C}\left(\mathbb{R}^{2 N}, \mathbb{R}^{N}\right), \sigma_{\Theta} \widehat{\Theta}\right)$. In particular consider $u_{0} \in B_{1} \cap D \subset \mathbb{R}^{N}$ and define the functions

$$
\begin{aligned}
h(t, x, u) & =f(t, x, u)-f\left(t, x, u_{0}\right) \quad \text { and } \\
h_{n}(t, x, u) & =f_{n}(t, x, u)-f_{n}\left(t, x, u_{0}\right), \forall n \in \mathbb{N} .
\end{aligned}
$$

Notice that the set $\left\{h_{n} \mid n \in \mathbb{N}\right\} \cup\{h\}$ has $L_{\text {loc }}^{1}$-bounded $m$-bounds. Indeed, for any $j \in \mathbb{N}$, considered $(x, u) \in B_{j} \subset \mathbb{R}^{2 N}$ one has that for any $n \in \mathbb{N}$

$$
\left|h_{n}(t, x, u)\right|=\left|f_{n}(t, x, u)-f_{n}\left(t, x, u_{0}\right)\right| \leq l_{n}^{j}(t)\left|u-u_{0}\right| \leq(j+1) l_{n}^{j}(t)
$$

for almost every $t \in \mathbb{R}$, where $l_{n}^{j}(\cdot) \in L_{l o c}^{1}$ is the optimal $l_{2}$-bound for $f_{n}$ on $B_{j} \subset \mathbb{R}^{2 N}$. Additionally, one can repeat analogous arguments for the function $h$ obtaining

$$
|h(t, x, u)| \leq(j+1) l^{j}(t) \text { for a.e. } t \in \mathbb{R},
$$

where $l^{j}(\cdot) \in L_{l o c}^{1}$ is the optimal $l_{2}$-bound for $f$ on $B_{j} \subset \mathbb{R}^{2 N}$. Thus, the $L_{l o c}^{1}$ boundedness of the $l_{2}$-bounds for the set $E$ gives $L_{l o c}^{1}$-bounded $m$-bounds for $\left\{h_{n} \mid n \in \mathbb{N}\right\} \cup\{h\}$. Moreover, we also have that the same set has $L_{l o c}^{1}$-bounded $l_{2}$-bounds. Indeed, for any $j \in \mathbb{N}$, considered $\left(x, u_{1}\right),\left(x, u_{2}\right) \in B_{j} \subset \mathbb{R}^{2 N}$ one has that for every $n \in \mathbb{N}$

$$
\left|h_{n}\left(t, x, u_{1}\right)-h_{n}\left(t, x, u_{2}\right)\right|=\left|f_{n}\left(t, x, u_{1}\right)-f_{n}\left(t, x, u_{2}\right)\right| \leq l_{n}^{j}(t)\left|u_{1}-u_{2}\right|,
$$

for almost every $t \in \mathbb{R}$, and one can repeat analogous arguments for the function $h$ obtaining

$$
\left|h\left(t, x, u_{1}\right)-h\left(t, x, u_{2}\right)\right| \leq l^{j}(t)\left|u_{1}-u_{2}\right| \quad \text { for a.e. } t \in \mathbb{R} .
$$

Therefore, from the $L_{l o c}^{1}$-boundedness of the $l_{2}$-bounds for the set $E$ one has that $\left\{h_{n} \mid n \in \mathbb{N}\right\} \cup\{h\}$ also has $L_{\text {loc }}^{1}$-bounded $l_{2}$-bounds. Finally notice that $\left(h_{n}\right)_{n \in \mathbb{N}}$ converges to $h$ in $\left(\mathfrak{S C}\left(\mathbb{R}^{2 N}, \mathbb{R}^{N}\right), \sigma_{\Theta D}\right)$. Indeed, fixed $I=\left[q_{1}, q_{2}\right]$, with $q_{1}, q_{2} \in \mathbb{Q}$ and $j \in \mathbb{N}$, for any $x(\cdot) \in \mathcal{K}_{j}^{I}$ and $u \in D$ we have

$$
\begin{aligned}
& \left|\int_{I}\left[h_{n}(t, x(t), u)-h(t, x(t), u)\right] d t\right| \\
& \quad \leq\left|\int_{I}\left[f_{n}(t, x(t), u)-f(t, x(t), u)\right] d t\right|+\left|\int_{I}\left[f\left(t, x(t), u_{0}\right)-f_{n}\left(t, x(t), u_{0}\right)\right] d t\right|
\end{aligned}
$$

and since $\left(f_{n}\right)_{n \in \mathbb{N}}$ converges to $f$ in $\left(\mathfrak{S C}\left(\mathbb{R}^{2 N}, \mathbb{R}^{N}\right), \sigma_{\Theta D}\right)$, then the integrals on the right-hand side of the previous inequality go to zero as $n \rightarrow \infty$. Therefore, 
the assumptions of step 1 apply to the set $E_{1}=\left\{h_{n} \mid n \in \mathbb{N}\right\} \cup\{h\}$ and thus one has that $\left(h_{n}\right)_{n \in \mathbb{N}}$ converges to $h$ in $\left(\mathfrak{S C}\left(\mathbb{R}^{2 N}, \mathbb{R}^{N}\right), \sigma_{\Theta \widehat{\Theta}}\right)$. Hence, for each interval $I=\left[q_{1}, q_{2}\right]$, with $q_{1}, q_{2} \in \mathbb{Q}$ and for each $j \in \mathbb{N}$ one has

$$
\begin{aligned}
& \sup _{x(\cdot) \in \mathcal{K}_{j}^{I}, u(\cdot) \in \widehat{\mathcal{K}}_{j}^{I-1}}\left|\int_{I}\left[f_{n}(t, x(t), u(t))-f(t, x(t), u(t))\right] d t\right| \\
& \leq \sup _{x(\cdot) \in \mathcal{K}_{j}^{I}, u(\cdot) \in \widehat{\mathcal{K}}_{j}^{I-1}}\left|\int_{I}\left[h_{n}(t, x(t), u(t))-h(t, x(t), u(t))\right] d t\right| \\
& \quad+\sup _{x(\cdot) \in \mathcal{K}_{j}^{I}}\left|\int_{I}\left[f_{n}\left(t, x(t), u_{0}\right)-f\left(t, x(t), u_{0}\right)\right] d t\right|
\end{aligned}
$$

and the right-hand side goes to zero as $n \rightarrow \infty$ because $\left(h_{n}\right)_{n \in \mathbb{N}}$ converges to $h$ in $\left(\mathfrak{S C}\left(\mathbb{R}^{2 N}, \mathbb{R}^{N}\right), \sigma_{\Theta} \widehat{\Theta}\right)$ and $\left(f_{n}\right)_{n \in \mathbb{N}}$ converges to $f$ in $\left(\mathfrak{S C}\left(\mathbb{R}^{2 N}, \mathbb{R}^{N}\right), \sigma_{\Theta D}\right)$. As a consequence $\left(f_{n}\right)_{n \in \mathbb{N}}$ converges to $f$ in $\left(\mathfrak{S} \mathfrak{C}\left(\mathbb{R}^{2 N}, \mathbb{R}^{N}\right), \sigma_{\Theta \widehat{\Theta}}\right)$ and, thus, all the topologies of type $\sigma_{D}$ and $\sigma_{\Theta}$ coincide on $E$.

Remark 5.10. As a consequence of Theorems 5.9 and 2.33, and recalling that $L_{l o c}^{1}$-bounded $l$-bounds imply $L_{l o c}^{1}$-bounded $l_{2}$-bounds, one immediately notice that all the weak topologies in the second chain of (5.4) coincide on the subsets of $\mathfrak{L} \mathfrak{C}\left(\mathbb{R}^{2 N}, \mathbb{R}^{N}\right)$ with $L_{\text {loc }}^{1}$-bounded $l$-bounds. Consequently, the results of relative compactness contained in Section 2.6 apply also to the weak hybrid topologies on the subsets of $\mathfrak{L} \mathfrak{C}\left(\mathbb{R}^{2 N}, \mathbb{R}^{N}\right)$ with $L_{\text {loc }}^{1}$-bounded l-bounds.

\subsection{Continuity of the semiflow}

This section will include some results allowing to construct a continuous skew product semiflow for Carathéodory DDEs. Depending on the properties on the vector field of the initial Carathéodory problem and on the topology used, several different results can be written.

\subsubsection{Continuity with respect to $\mathcal{T}_{B}$ and $\mathcal{T}_{D}$}

The first results of continuity for skew product semiflows that we present, take into account two topologies that have been introduced in Chapter 2: $\mathcal{T}_{B}$ and $\mathcal{T}_{D}$. Notice that the content of Section 2.7 already solves the problem of the continuity on the base for this topologies and thus we can directly face the continuity of the solutions with respect to initial data and vector fields.

Theorem 5.11. Consider a sequence $\left(f_{n}\right)_{n \in \mathbb{N}}$ in $\mathfrak{L} \mathfrak{C}\left(\mathbb{R}^{2 N}, \mathbb{R}^{N}\right)$ converging to $f$ in $\left(\mathfrak{L} \mathfrak{C}\left(\mathbb{R}^{2 N}, \mathbb{R}^{N}\right), \mathcal{T}_{B}\right)$ and $\left(\phi_{n}(\cdot)\right)_{n \in \mathbb{N}}$ in $\mathcal{C}$ converging uniformly to $\phi \in \mathcal{C}$. Then, with the notation of Theorem 5.1,

$$
x\left(\cdot, f_{n}, \phi_{n}\right) \stackrel{n \rightarrow \infty}{\longrightarrow} x(\cdot, f, \phi)
$$

uniformly in any $\left[T_{1}, T_{2}\right] \subset I_{f, \phi}$. 
Proof. Following the notation of Theorem 5.1, let $I_{f, \phi}$ be the maximal interval of definition of the solution of the delay differential equation $\dot{x}=f(t, x(t), x(t-1))$ with initial data $x(t)=\phi(t)$ for all $t \in[-1,0]$. Let us consider the interval $[-1, \beta]$ with $\beta<b_{f, \phi}$. Denote

$$
0<\rho=1+\max \left\{\left(\left\|\phi_{n}\right\|_{L^{\infty}([-1,0])}\right)_{n \in \mathbb{N}},\|x(\cdot, f, \phi)\|_{L^{\infty}([-1, \beta])}\right\},
$$

and define

$$
z_{n}(t)= \begin{cases}x\left(t, f_{n}, \phi_{n}\right), & \text { if }-1 \leq t<T_{n}, \\ x\left(T_{n}, f_{n}, \phi_{n}\right), & \text { if } T_{n} \leq t \leq \beta\end{cases}
$$

where $T_{n}=\sup \left\{t \in[-1, \beta]|| x\left(s, f_{n}, \phi_{n}\right) \mid \leq \rho, \forall s \in[-1, t]\right\}$. Notice that by (5.13) and by the continuity of $\left(x\left(\cdot, f_{n}, \phi_{n}\right)\right)_{n \in \mathbb{N}}$, we have that $T_{n}>0$ for any $n \in \mathbb{N}$. In particular notice that $\left(z_{n}(\cdot)\right)_{n \in \mathbb{N}}$ is uniformly bounded and let $j \in \mathbb{N}$ be so that $\rho<j$. If $t_{1}<t_{2}$, and in particular they are both in the same subinterval, either $[-1,0),\left[0, T_{n}\right)$ or $\left[T_{n}, \beta\right]$, then

$$
\left|z_{n}\left(t_{1}\right)-z_{n}\left(t_{2}\right)\right| \leq \begin{cases}\left|\phi_{n}\left(t_{1}\right)-\phi_{n}\left(t_{2}\right)\right| & \text { if } t_{1}, t_{2} \in[-1,0), \\ \left|\int_{t_{1}}^{t_{2}} f_{n}\left(s, z_{n}(s), z_{n}(s-1)\right) d s\right| & \text { if } t_{1}, t_{2} \in\left[0, T_{n}\right), \\ 0 & \text { if } t_{1}, t_{2} \in\left[T_{n}, \beta\right] .\end{cases}
$$

In order to study the equicontinuity of the functions $z_{n}(\cdot)$, with $n \in \mathbb{N}$, we can assume, without any loss of generality, that $t_{1}, t_{2}$ always belong to the same subinterval, either $[-1,0),\left[0, T_{n}\right)$ or $\left[T_{n}, \beta\right]$. Let us fix $\varepsilon>0$ and analyze each case separately.

Case 1: $t_{1}, t_{2} \in[-1,0)$. Since $\left(\phi_{n}(\cdot)\right)_{n \in \mathbb{N}}$ converges uniformly to $\phi(\cdot)$ on $[-1,0]$, there exists $n_{0} \in \mathbb{N}$ such that if $n>n_{0}$, then $\left\|\phi_{n}(\cdot)-\phi(\cdot)\right\|_{L^{\infty}([-1,0])}<\varepsilon / 2$. Using the continuity of the functions $\phi_{n}(\cdot)$ for $n=1, \ldots, n_{0}$, we find a $\delta_{1}>0$ such that for every $n=1, \ldots, n_{0}$ and $t_{1}, t_{2} \in[-1,0)$, if $\left|t_{1}-t_{2}\right|<\delta_{1}$, then $\left|\phi_{n}\left(t_{1}\right)-\phi_{n}\left(t_{2}\right)\right|<\varepsilon$. Therefore, irrespectively of $n \in \mathbb{N}$, if $t_{1}, t_{2} \in[-1,0)$ and $\left|t_{1}-t_{2}\right|<\delta_{1}$, we have $\left|\phi_{n}\left(t_{1}\right)-\phi_{n}\left(t_{2}\right)\right|<\varepsilon$.

Case 2: $t_{1}, t_{2} \in\left[0, T_{n}\right)$. Denote by $m_{f}(\cdot)$ the optimal $m$-bound of $f$ on $B_{2 j} \subset$ $\mathbb{R}^{2 N}$. Then, we have that

$$
\begin{aligned}
& \left|z_{n}\left(t_{1}\right)-z_{n}\left(t_{2}\right)\right| \leq\left|\int_{t_{1}}^{t_{2}} f_{n}\left(s, z_{n}(s), z_{n}(s-1)\right) d s\right| \\
& \quad \leq \int_{t_{1}}^{t_{2}}\left|f_{n}\left(s, z_{n}(s), z_{n}(s-1)\right)-f\left(s, z_{n}(s), z_{n}(s-1)\right)\right| d s+\int_{t_{1}}^{t_{2}} m_{f}(s) d s \\
& \quad \leq \int_{0}^{\beta}\left|f_{n}\left(s, z_{n}(s), z_{n}(s-1)\right)-f\left(s, z_{n}(s), z_{n}(s-1)\right)\right| d s+\int_{t_{1}}^{t_{2}} m_{f}(s) d s .
\end{aligned}
$$

For the given $\varepsilon>0$, since $\left(f_{n}\right)_{n \in \mathbb{N}}$ converges to $f$ in $\left(\mathfrak{L C}\left(\mathbb{R}^{2 N}, \mathbb{R}^{N}\right), T_{B}\right)$ and 
$\left(z_{n}(\cdot)\right)_{n \in \mathbb{N}}$ is bounded, one has that there exists $n_{1} \in \mathbb{N}$ such that, if $n>n_{1}$ then

$$
\sup _{(x(\cdot), u(\cdot)) \in C\left([0, \beta], B_{2 j}\right)} \int_{0}^{\beta}\left|f_{n}(s, x(s), u(s))-f(s, x(s), u(s))\right| d s<\frac{\varepsilon}{2} .
$$

On the other side, for $n=1, \ldots, n_{1}$, by the continuity of the integral, there exists a $\delta_{2}>0$ such that if $t_{1}, t_{2} \in\left[0, T_{n}\right)$ and $\left|t_{1}-t_{2}\right|<\delta_{2}$, then

$$
\int_{t_{1}}^{t_{2}}\left|f_{n}\left(s, z_{n}(s), z_{n}(s-1)\right)-f\left(s, z_{n}(s), z_{n}(s-1)\right)\right| d s<\frac{\varepsilon}{2}, \quad n=1, \ldots, n_{1},
$$

and also

$$
\int_{t_{1}}^{t_{2}} m_{f}(s) d s<\frac{\varepsilon}{2}
$$

Hence, irrespectively of $n \in \mathbb{N}$, if $t_{1}, t_{2} \in\left[0, T_{n}\right)$ and $\left|t_{1}-t_{2}\right|<\delta_{2}$, we have that

$$
\left|\int_{t_{1}}^{t_{2}} f_{n}\left(s, z_{n}(s), z_{n}(s-1)\right) d s\right|<\varepsilon
$$

Case 3: $t_{1}, t_{2} \in\left[T_{n}, \beta\right]$. This case is trivial.

Therefore, taking $\delta=\min \left\{\delta_{1}, \delta_{2}\right\}$ we have that for all $n \in \mathbb{N}$ if $\left|t_{1}-t_{2}\right|<\delta$ then $\left|z_{n}\left(t_{1}\right)-z_{n}\left(t_{2}\right)\right|<\varepsilon$, i.e. $\left(z_{n}(\cdot)\right)_{n \in \mathbb{N}}$ is equicontinuous. Thus, applying AscoliArzelá's theorem we obtain a subsequence, that we keep denoting with the same indexes, converging uniformly to some $z(\cdot) \in C([-1, \beta])$. We claim that $z(\cdot)$ coincides with $x(\cdot, f, \phi)$ on $[-1, \beta]$. Trivially, $z(\cdot)=\phi(\cdot)$ on $[-1,0]$. Define

$$
T_{0}=\sup \{t \in[-1, \beta]|| z(s) \mid<\rho-1 / 2 \quad \forall s \in[-1, t]\}
$$

and notice that $T_{0}>0$ because $\left(\phi_{n}(\cdot)\right)_{n \in \mathbb{N}}$ converges uniformly to $\phi$ in $[-1,0]$ and $z(\cdot)$ is continuous. Moreover, from the uniform convergence of $\left(z_{n}(\cdot)\right)_{n \in \mathbb{N}}$ to $z(\cdot)$ in $[-1, \beta]$, we have that there exists $n_{0} \in \mathbb{N}$ such that if $n>n_{0}$, then

$$
\left|z_{n}(t)\right|<\rho-1 / 4 \quad \forall t \in\left[-1, T_{0}\right]
$$

Therefore, for any $t \in\left[-1, T_{0}\right]$ and for any $n>n_{0}$ one has $z_{n}(t)=x\left(t, f_{n}, \phi_{n}\right)$, i.e., if $n>n_{0}$, then

$$
z_{n}(t)= \begin{cases}\phi_{n}(t) & \text { if } t \in[-1,0) \\ \phi_{n}(0)+\int_{0}^{t} f_{n}\left(s, z_{n}(s), z_{n}(s-1)\right) d s & \text { if } t \in\left[0, T_{0}\right] .\end{cases}
$$

Now consider the bounded set $\mathcal{B} \subset C\left(\left[0, T_{0}\right], B_{j}\right) \times C\left(\left[-1, T_{0}-1\right], B_{j}\right)$ given by

$$
\mathcal{B}=\left\{\left(z_{n}(\cdot), z_{n}(\cdot-1)\right) \mid n \in \mathbb{N}\right\} \cup\{(z(\cdot), z(\cdot-1))\}
$$


Then, for all $t \in\left[0, T_{0}\right]$, we have that

$$
\begin{gathered}
\left|\int_{0}^{t} f_{n}\left(s, z_{n}(s), z_{n}(s-1)\right) d s-\int_{0}^{t} f(s, z(s), z(s-1)) d s\right| \\
\leq \int_{0}^{t}\left|f_{n}\left(s, z_{n}(s), z_{n}(s-1)\right)-f\left(s, z_{n}(s), z_{n}(s-1)\right)\right| d s \\
\quad+\int_{0}^{t}\left|f\left(s, z_{n}(s), z_{n}(s-1)\right)-f(s, z(s), z(s-1))\right| d s \\
\leq \sup _{(\zeta(\cdot), \zeta(\cdot-1)) \in \mathcal{B}} \int_{0}^{t}\left|f_{n}(s, \zeta(s), \zeta(s-1))-f(s, \zeta(s), \zeta(s-1))\right| d s \\
\quad+2\left\|z_{n}(\cdot)-z(\cdot)\right\|_{L^{\infty}([-1, \beta])} \int_{0}^{t} l_{f}(s) d s,
\end{gathered}
$$

where $l_{f}(\cdot) \in L_{\text {loc }}^{1}$ is the optimal $l$-bound for $f$ on $B_{2 j} \subset \mathbb{R}^{2 N}$. In particular, when $t \in\left[0, T_{0}\right] \cap \mathbb{Q}$, the right-hand side of the previous inequality goes to zero as $n \rightarrow \infty$ because $\left(f_{n}\right)_{n \in \mathbb{N}}$ converges to $f$ in $\left(\mathfrak{L} \mathfrak{C}\left(\mathbb{R}^{2 N}, \mathbb{R}^{N}\right), T_{B}\right)$ and $\left(z_{n}(\cdot)\right)_{n \in \mathbb{N}}$ converges uniformly to $z(\cdot)$ in $[-1, \beta]$. Then, thanks to $(5.16)$ and to the fact that $\left(\phi_{n}(\cdot)\right)_{n \in \mathbb{N}}$ converges uniformly to $\phi \in \mathcal{C}$, passing to the limit in (5.15), we have that

$$
z(t)= \begin{cases}\phi(t) & \text { if } t \in[-1,0) \\ \phi(0)+\int_{0}^{t} f(s, z(s), z(s-1)) d s & \text { if } t \in\left[0, T_{0}\right] \cap \mathbb{Q} .\end{cases}
$$

As a matter of fact, the formula in the second line of (5.17) holds for any $t \in\left[0, T_{0}\right]$ thanks to the continuity of $z(\cdot)$ and the continuity of the integral. Therefore, $z(\cdot)$ coincides with $x\left(\cdot, f, x_{0}\right)$ on $\left[0, T_{0}\right]$.

Moreover, we claim that $T_{0}=\beta$. Otherwise, by (5.14) and by the continuity of $z(\cdot)$, one would have $\left|z\left(T_{0}\right)\right|=\left|x\left(T_{0}, f, \phi\right)\right|=\rho-1 / 2$, which contradicts (5.13). Hence, $T_{0}=\beta$, as claimed, and thus for any $t \in[0, \beta]$ we have that $x(t, f, \phi)=z(t)$ and $x\left(t, f_{n}, \phi_{n}\right)=z_{n}(t)$ for any $n \in \mathbb{N}$, which concludes the proof.

Theorem 5.12. Consider $E \subset \mathfrak{L C}\left(\mathbb{R}^{2 N}, \mathbb{R}^{N}\right)$ with $L_{\text {loc }}^{p}$-bounded l-bounds. If $\left(f_{n}\right)_{n \in \mathbb{N}}$ is a sequence in $E$ converging to $f$ in $\left(\mathfrak{L} \mathfrak{C}\left(\mathbb{R}^{2 N}, \mathbb{R}^{N}\right), \mathcal{T}_{D}\right)$ and $\left(\phi_{n}(\cdot)\right)_{n \in \mathbb{N}}$ is a sequence in $\mathcal{C}$ converging uniformly to $\phi \in \mathcal{C}$, then, with the notation of Theorem 5.1,

$$
x\left(\cdot, f_{n}, \phi_{n}\right) \stackrel{n \rightarrow \infty}{\longrightarrow} x(\cdot, f, \phi)
$$

uniformly in any $\left[T_{1}, T_{2}\right] \subset I_{f, \phi}$.

Proof. This result is a direct consequence of Theorem 5.11 and Theorem 2.31.

As a consequence of Theorem 5.11 and Theorem 5.12, together with Remark 2.41 and Corollary 2.42, one can deduce a first result of continuity of the skew-product semiflow composed of the time translation of the initial vector field and the solutions of the respective delay differential equation. 
Consider $f \in \mathfrak{L} \mathfrak{C}\left(\mathbb{R}^{2 N}, \mathbb{R}^{N}\right)$. With the notation introduced in Theorem 5.2, let us denote by $\mathcal{U}_{B}$ the subset of $\mathbb{R} \times \operatorname{Hull}_{\left(\mathfrak{L C}, \mathcal{T}_{B}\right)}(f) \times \mathcal{C}$ given by

$$
\mathcal{U}_{B}=\bigcup_{\substack{g \in \operatorname{Hull}_{\left(\mathfrak{s} \mathfrak{C}, \mathcal{T}_{B}\right)}(f) \\ \phi \in \mathcal{C}}}\left\{(t, g, \phi) \mid t \in I_{g, \phi}\right\},
$$

and by $\mathcal{U}_{D}$ the subset of $\mathbb{R} \times \operatorname{Hull}_{\left(\mathfrak{L C}, \mathcal{T}_{B}\right)}(f) \times \mathcal{C}$ given by

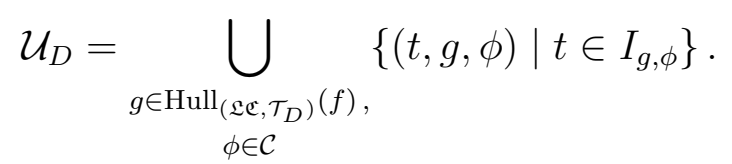

Theorem 5.13. Consider $f \in \mathfrak{L} \mathfrak{C}\left(\mathbb{R}^{2 N}, \mathbb{R}^{N}\right)$.

(i) The set $\mathcal{U}_{B}$ is open in $\mathbb{R} \times \operatorname{Hull}_{\left(\mathfrak{L C}, \mathcal{T}_{B}\right)}(f) \times \mathcal{C}$ and the map

$$
\begin{array}{rlr}
\Pi: \mathcal{U}_{B} \subset \mathbb{R} \times \operatorname{Hull}_{\left(\mathfrak{L C}, \mathcal{T}_{B}\right)}(f) \times \mathcal{C} & \rightarrow & \operatorname{Hull}_{\left(\mathfrak{L C}, \mathcal{T}_{B}\right)}(f) \times \mathcal{C} \\
(t, g, \phi) & \mapsto & \left(g_{t}, x_{t}(\cdot, g, \phi)\right)
\end{array}
$$

defines a local continuous skew-product semiflow on $\operatorname{Hull}_{\left(\mathfrak{L C}, \mathcal{T}_{B}\right)}(f) \times \mathcal{C}$.

(ii) If additionally $f$ admits $L_{\text {loc }}^{p}$-bounded l-bounds, then the set $\mathcal{U}_{D}$ is open in $\mathbb{R} \times \operatorname{Hull}_{\left(\mathfrak{L C}, \mathcal{T}_{D}\right)}(f) \times \mathcal{C}$ and the map

$$
\begin{array}{rlc}
\Pi: \mathcal{U}_{D} \subset \mathbb{R} \times \operatorname{Hull}_{\left(\mathfrak{L C}, \mathcal{T}_{D}\right)}(f) \times \mathcal{C} & \rightarrow & \operatorname{Hull}_{\left(\mathfrak{L C}, \mathcal{T}_{D}\right)}(f) \times \mathcal{C} \\
(t, g, \phi) & \mapsto & \left(g_{t}, x_{t}(\cdot, g, \phi)\right)
\end{array}
$$

defines a local continuous skew-product semiflow on $\operatorname{Hull}_{\left(\mathfrak{L C}, \mathcal{T}_{D}\right)}(f) \times \mathcal{C}$.

Proof. The proof is a direct consequence of Corollary 2.42, of Theorem 5.11 and of Theorem 5.12.

\subsubsection{Continuity with respect to $\mathcal{T}_{\Theta B}, \mathcal{T}_{\Theta D}$ and $\sigma_{\Theta D}$}

As one can easily check, the results contained in the previous subsection are mainly based on the ones presented in Chapter 3. As follows, we want to inquire into the new topologies presented in Definition 5.4 and see which advantages they have with respect to $\mathcal{T}_{B}$ and $\mathcal{T}_{D}$. As a first step we need to prove the continuity on the base for such hybrid topologies.

Theorem 5.14. Let $\Theta$ and $\widehat{\Theta}$ be suitable sets of moduli of continuity and $D$ a dense countable subset of $\mathbb{R}^{N}$.

(i) The map

$$
\begin{gathered}
\Pi: \mathbb{R} \times \mathfrak{L} \mathfrak{C}\left(\mathbb{R}^{2 N}, \mathbb{R}^{N}\right) \rightarrow \mathfrak{L} \mathfrak{C}\left(\mathbb{R}^{2 N}, \mathbb{R}^{N}\right), \quad(t, f) \mapsto \Pi(t, f)=f_{t}, \\
\text { defines a continuous flow on }\left(\mathfrak{L} \mathfrak{C}\left(\mathbb{R}^{2 N}, \mathbb{R}^{N}\right), \mathcal{T}\right) \text {, with } \mathcal{T} \in\left\{\mathcal{T}_{\Theta B}, \mathcal{T}_{\Theta \widehat{\Theta}}, \mathcal{T}_{\Theta D}\right\} .
\end{gathered}
$$


(ii) Moreover, if $E \subset \mathfrak{L} \mathfrak{C}\left(\mathbb{R}^{2 N}, \mathbb{R}^{N}\right)$ has $L_{\text {loc }}^{1}$-equicontinuous $m$-bounds and it is such that $f \in E$ implies $f_{t} \in E$ for all $t \in \mathbb{R}$, then the map

$$
\Pi: \mathbb{R} \times E \rightarrow E, \quad(t, f) \mapsto \Pi(t, f)=f_{t},
$$

defines a continuous flow on $(E, \sigma)$, where $\sigma \in\left\{\sigma_{\Theta \Theta}, \sigma_{\Theta D}\right\}$.

Proof. Let us firstly deal with the case $\left(\mathfrak{L} \mathfrak{C}\left(\mathbb{R}^{2 N}, \mathbb{R}^{N}\right), \mathcal{T}_{\Theta} \widehat{\Theta}\right)$. We separately deal with the continuity with respect to $f$ and with respect to $t$, and eventually gather them together.

Let $\left(f_{n}\right)_{n \in \mathbb{N}}$ be a sequence in $\mathfrak{L} \mathfrak{C}\left(\mathbb{R}^{2 N}, \mathbb{R}^{N}\right)$ converging to some function $f$ in $\left(\mathfrak{L} \mathfrak{C}\left(\mathbb{R}^{2 N}, \mathbb{R}^{N}\right), \mathcal{T}_{\Theta} \widehat{\Theta}\right)$. We prove that $\left(f_{n}\right)_{t} \rightarrow f_{t}$ in $\left(\mathfrak{L} \mathfrak{C}\left(\mathbb{R}^{2 N}, \mathbb{R}^{N}\right), \mathcal{T}_{\Theta} \widehat{\Theta}\right)$ as $n \rightarrow \infty$, uniformly for $t$ in a compact interval. Consider $I=\left[p_{1}, p_{2}\right]$ and $J=$ $\left[q_{1}, q_{2}\right]$ such that $p_{1}, p_{2}, q_{1}, q_{2} \in \mathbb{Q}$ and fix $t \in J$. Moreover, for any $j \in \mathbb{N}$ consider $\mathcal{K}_{j}^{I}, \mathcal{K}_{j}^{I+J}, \widehat{\mathcal{K}}_{j}^{I-1}$ and $\widehat{\mathcal{K}}_{j}^{I-1+J}$ as in Definition 5.4. Notice that $x(\cdot) \in \mathcal{K}_{j}^{I}$ implies $x(\cdot-t) \in \mathcal{K}_{j}^{I+J}$ up to a suitable extension by constants of the function $x(\cdot-t)$ in $I+J$, and that similar reasonings apply to $\widehat{\mathcal{K}}_{j}^{I-1}$ and $\widehat{\mathcal{K}}_{j}^{I-1+J}$. Then

$$
\begin{aligned}
& \sup _{x(\cdot) \in \mathcal{K}_{j}^{I}, u(\cdot) \in \widehat{\mathcal{K}}_{j}^{I-1}} \int_{I}\left|\left(f_{n}\right)_{t}(s, x(s), u(s-1))-f_{t}(s, x(s), u(s-1))\right| d s \\
& =\sup _{x(\cdot) \in \mathcal{K}_{j}^{I}, u(\cdot) \in \widehat{\mathcal{K}}_{j}^{I-1}} \int_{I+t}\left|f_{n}(r, x(r-t), u(r-t-1))-f(r, x(r-t), u(r-t-1))\right| d r \\
& \leq \sup _{x(\cdot) \in \mathcal{K}_{j}^{I+J}, u(\cdot) \in \widehat{\mathcal{K}}_{j}^{I+J-1}} \int_{I+J}\left|f_{n}(r, x(r), u(r-1))-f(r, x(r), u(r-1))\right| d r,
\end{aligned}
$$

and taking the limit as $n \rightarrow \infty$ we have that the right-hand side of (5.18) goes to zero, since $f_{n} \stackrel{n \rightarrow \infty}{\longrightarrow} f$ in $\left(\mathfrak{L C}\left(\mathbb{R}^{2 N}, \mathbb{R}^{N}\right), \mathcal{T}_{\Theta} \widehat{\Theta}\right)$. Therefore, one obtains the aimed continuity in the first variable, uniformly for $t$ in a compact set. Notice that, with an analogous reasoning, one obtains the continuity of $\Pi$ with respect to the second variable, uniformly for $t$ in a compact set, for any of the strong or weak hybrid topologies cited in the statement.

Next, we prove the continuity with respect to the first variable; in other words that the map $t \mapsto f_{t}$ of $\mathbb{R}$ into $\left(\mathfrak{L} \mathfrak{C}\left(\mathbb{R}^{2 N}, \mathbb{R}^{N}\right), \mathcal{T}_{\Theta} \widehat{\Theta}\right)$ is continuous. Consider $f \in \mathfrak{L} \mathfrak{C}\left(\mathbb{R}^{2 N}, \mathbb{R}^{N}\right)$, an interval $I=[a, b]$, with $a, b \in \mathbb{Q}$, and $t \in \mathbb{R}$ fixed. We aim to prove that for any couple of compact sets $\mathcal{K}_{j}^{I}$ and $\widehat{\mathcal{K}}_{j}^{I-1}$ as in Definition 5.4, we have that

$$
\lim _{\tau \rightarrow 0} \sup _{x(\cdot) \in \mathcal{K}_{j}^{I}, u(\cdot) \in \widehat{\mathcal{K}}_{j}^{I-1}} \int_{I}\left|f_{t+\tau}(s, x(s), u(s-1))-f_{t}(s, x(s), u(s-1))\right| d s=0 .
$$

Firstly, let us fix $x(\cdot) \in \mathcal{K}_{j}^{I}$ and $u(\cdot) \in \widehat{\mathcal{K}}_{j}^{I-1}$ and prove that if $\tau_{n} \stackrel{n \rightarrow \infty}{\longrightarrow} 0$ then

$$
\lim _{n \rightarrow \infty} \int_{I}\left|f_{t+\tau_{n}}(s, x(s), u(s-1))-f_{t}(s, x(s), u(s-1))\right| d s=0 .
$$


Notice that, for all $t \in \mathbb{R}, f_{t}(\cdot, x(\cdot), y(\cdot-1)) \in L^{1}\left(I, \mathbb{R}^{M}\right)$ and consider the operator $T_{\tau}: L^{1}\left(I, \mathbb{R}^{M}\right) \rightarrow L^{1}\left(\mathbb{R}, \mathbb{R}^{M}\right)$, such that $g(\cdot) \mapsto T_{\tau} g(\cdot)$, where $T_{\tau} g(\cdot)$ is defined by

$$
T_{\tau} g(s)=\left\{\begin{array}{cl}
g(s+\tau), & \text { if } s+\tau \in I \\
0, & \text { otherwise }
\end{array}\right.
$$

By the continuity of translations in $L^{1}(I)$, see Castillo and Rafeiro [17, Theorem 3.58, p.101], we have that, if $\left|\tau_{n}\right| \rightarrow 0$ as $n \rightarrow \infty$, then for a given $\varepsilon>0$ there exists $\delta>0$ such that

$$
\sup _{\left|\tau_{n}\right|<\delta}\left\|T_{\tau_{n}} f_{t}(\cdot, x(\cdot), u(\cdot-1))-f_{t}(\cdot, x(\cdot), u(\cdot-1))\right\|_{L^{1}(I)} \leq \varepsilon .
$$

Now, for any $n \in \mathbb{N}$ define $a_{n}=\max \left\{a, a-\tau_{n}\right\}$ and $b_{n}=\min \left\{b, b-\tau_{n}\right\}$, and consider $n_{0} \in \mathbb{N}$ so that for any $n>n_{0}$ we have $\left|\tau_{n}\right|<\delta$. Therefore, for any $n>n_{0}$ the following chain of inequalities holds

$$
\begin{aligned}
\| f_{t+\tau_{n}}(\cdot, & x(\cdot), u(\cdot-1))-f_{t}(\cdot, x(\cdot), u(\cdot-1)) \|_{L^{1}(I)} \\
\leq \| & T_{\tau_{n}} f_{t}(\cdot, x(\cdot), u(\cdot-1))-f_{t}(\cdot, x(\cdot), u(\cdot-1)) \|_{L^{1}(I)} \\
& +\left\|f_{t+\tau_{n}}(\cdot, x(\cdot), u(\cdot-1))-T_{\tau_{n}} f_{t}(\cdot, x(\cdot), u(\cdot-1))\right\|_{L^{1}(I)} \\
\leq \varepsilon & +\left\|f_{t+\tau_{n}}(\cdot, x(\cdot), u(\cdot-1))-T_{\tau_{n}} f_{t}(\cdot, x(\cdot), u(\cdot-1))\right\|_{L^{1}(I)} \\
\leq \varepsilon & +\int_{a_{n}}^{b_{n}}\left|f_{t}\left(s+\tau_{n}, x(s), u(s-1)\right)-f_{t}\left(s+\tau_{n}, x\left(s+\tau_{n}\right), u\left(s+\tau_{n}-1\right)\right)\right| d s \\
& +\int_{a}^{a_{n}}\left|f_{t}\left(s+\tau_{n}, x(s), u(s-1)\right)\right| d s+\int_{b_{n}}^{b}\left|f_{t}\left(s+\tau_{n}, x(s), u(s-1)\right)\right| d s \\
\leq \varepsilon & +\int_{a_{n}+\tau_{n}}^{b_{n}+\tau_{n}}\left|f_{t}\left(r, x\left(r-\tau_{n}\right), u\left(r-\tau_{n}-1\right)\right)-f_{t}(r, x(r), u(r-1))\right| d r \\
& +\int_{a+\tau_{n}}^{a_{n}+\tau_{n}}\left|f_{t}\left(r, x\left(r-\tau_{n}\right), u\left(r-\tau_{n}-1\right)\right)\right| d r \\
& +\int_{b_{n}+\tau_{n}}^{b+\tau_{n}}\left|f_{t}\left(r, x\left(r-\tau_{n}\right), u\left(r-\tau_{n}-1\right)\right)\right| d r=\varepsilon+I_{1}+I_{2}+I_{3} .
\end{aligned}
$$

As regards $I_{1}$, notice that, up to extending the functions $x(\cdot), u(\cdot),\left(x\left(\cdot-\tau_{n}\right)\right)_{n \in \mathbb{N}}$ and $\left(u\left(\cdot-\tau_{n}-1\right)\right)_{n \in \mathbb{N}}$ by constants to an interval $J$ containing $I+[-\delta, \delta]$ we have that

$$
\begin{aligned}
I_{1} & \leq \int_{J}\left|f_{t}\left(r, x\left(r-\tau_{n}\right), u\left(r-\tau_{n}-1\right)\right)-f_{t}(r, x(r), u(r-1))\right| d r \\
& \leq \int_{J} l_{t}^{2 j}(r)\left|\left(x\left(r-\tau_{n}\right), u\left(r-\tau_{n}-1\right)\right)-(x(r), u(r-1))\right| d r \\
& \leq\left\|\left(x\left(\cdot-\tau_{n}\right), u\left(\cdot-\tau_{n}-1\right)\right)-(x(\cdot), u(\cdot-1))\right\|_{L^{\infty}(J)} \int_{J} l_{t}^{2 j}(r) d r
\end{aligned}
$$


where $l^{2 j}(\cdot)$ denotes the optimal $l$-bound for $f$ on $B_{2 j} \subset \mathbb{R}^{2 N}$. The right-hand side of equation (5.21) goes to zero as $n \rightarrow \infty$, due to the fact that

$$
\left\|\left(x\left(\cdot-\tau_{n}\right), u\left(\cdot-\tau_{n}-1\right)\right)-(x(\cdot), u(\cdot-1))\right\|_{L^{\infty}(J)} \stackrel{n \rightarrow \infty}{\longrightarrow} 0 .
$$

As regards $I_{2}$, let $m^{2 j}$ be the optimal $m$-bound of $f$ on $B_{2 j}$ and notice that the following chain of inequalities holds

$$
\left.I_{2} \leq \int_{a-\left|\tau_{n}\right|}^{a}\left|f_{t}\left(r, x\left(r-\tau_{n}\right), u\left(r-\tau_{n}-1\right)\right)\right| d r \leq \int_{a-\left|\tau_{n}\right|}^{a} m_{t}^{2 j}(r)\right) d r,
$$

and the integral on the right-hand side goes to zero as $n \rightarrow \infty$, thanks to the absolute continuity of the Lebesgue integral. Similar reasonings apply to $I_{3}$. Therefore, for any fixed $t \in \mathbb{R}, x(\cdot) \in \mathcal{K}_{j}^{I}$ and $u(\cdot) \in \widehat{\mathcal{K}}_{j}^{I-1}$ we obtain the limit in (5.20). Next we check that such a convergence is uniform in $\mathcal{K}_{j}^{I}$ and $\widehat{\mathcal{K}}_{j}^{I-1}$. Otherwise, it would exist an $\varepsilon>0$, a sequence $\left(x_{n}(\cdot)\right)_{n \in \mathbb{N}}$ in $\mathcal{K}_{j}^{I}$, a sequence $\left(u_{n}(\cdot)\right)_{n \in \mathbb{N}}$ in $\widehat{\mathcal{K}}_{j}^{I-1}$, and a sequence $\left(\tau_{n}\right)_{n \in \mathbb{N}}$ in $\mathbb{R}$ converging to 0 , such that for all $n \in \mathbb{N}$

$$
\int_{I}\left|f_{t+\tau_{n}}\left(s, x_{n}(s), u_{n}(s-1)\right)-f_{t}\left(s, x_{n}(s), u_{n}(s-1)\right)\right| d s>\varepsilon .
$$

However, being $\mathcal{K}_{j}^{I}$ and $\widehat{\mathcal{K}}_{j}^{I-1}$ compact, there exist subsequences of $\left(x_{n}(\cdot)\right)_{n \in \mathbb{N}}$ and $\left(u_{n}(\cdot)\right)_{n \in \mathbb{N}}$, which we keep denoting with the same indexes, converging uniformly to some $x(\cdot) \in \mathcal{K}_{j}^{I}$ and $u(\cdot) \in \widehat{\mathcal{K}}_{j}^{I-1}$, respectively, as $n \rightarrow \infty$. From (5.20), there exists $n_{0} \in \mathbb{N}$ such that, if $n>n_{0}$, then

$$
\left\|f_{t+\tau_{n}}(\cdot, x(\cdot), u(\cdot-1))-f_{t}(\cdot, x(\cdot), u(\cdot-1))\right\|_{L^{1}(I)}<\frac{\varepsilon}{2} .
$$

Then, recalling that $l^{2 j}(\cdot)$ denotes the optimal $l$-bound for $f$ on $B_{2 j} \subset \mathbb{R}^{2 N}$, considered $\delta>0$ such that $\left|\tau_{n}\right|<\delta$ for all $n \in \mathbb{N}$, for all $n>n_{0}$ we can write

$$
\begin{aligned}
& \varepsilon<\left\|f_{t+\tau_{n}}\left(\cdot, x_{n}(\cdot), u_{n}(\cdot-1)\right)-f_{t}\left(\cdot, x_{n}(\cdot), u_{n}(\cdot-1)\right)\right\|_{L^{1}(I)} \\
& \leq\left\|f_{t+\tau_{n}}\left(\cdot, x_{n}(\cdot), u_{n}(\cdot-1)\right)-f_{t+\tau_{n}}(\cdot, x(\cdot), u(\cdot-1))\right\|_{L^{1}(I)} \\
& \quad+\left\|f_{t+\tau_{n}}(\cdot, x(\cdot), u(\cdot-1))-f_{t}(\cdot, x(\cdot), u(\cdot-1))\right\|_{L^{1}(I)} \\
& \quad+\left\|f_{t}(\cdot, x(\cdot), u(\cdot-1))-f_{t}\left(\cdot, x_{n}(\cdot), u_{n}(\cdot-1)\right)\right\|_{L^{1}(I)} \\
& \leq \varepsilon / 2+\left\|\left(x_{n}(\cdot), u_{n}(\cdot-1)\right)-(x(\cdot), u(\cdot-1))\right\|_{L^{\infty}(I)}\left[\int_{I} l_{t+\tau_{n}}^{2 j}(s) d s+\int_{I} l_{t}^{2 j}(s) d s\right] \\
& \leq \varepsilon / 2+\left\|\left(x_{n}(\cdot), u_{n}(\cdot-1)\right)-(x(\cdot), u(\cdot-1))\right\|_{L^{\infty}(I)} 2 \int_{I+[-\delta, \delta]} l_{t}^{2 j}(s) d s .
\end{aligned}
$$

Moreover, since $\left(x_{n}(\cdot)\right)_{n \in \mathbb{N}}$ and $\left(u_{n}(\cdot)\right)_{n \in \mathbb{N}}$ converge uniformly to $x(\cdot) \in \mathcal{K}_{j}^{I}$ and $u(\cdot) \in \widehat{\mathcal{K}}_{j}^{I-1}$, respectively, then there exists $n_{1} \in \mathbb{N}$ such that, if $n>n_{1}$, one has

$$
\left\|\left(x_{n}(\cdot), u_{n}(\cdot-1)\right)-(x(\cdot), u(\cdot-1))\right\|_{L^{\infty}(I)}<\frac{\varepsilon}{2 C},
$$


where $C=2 \int_{I+[-\delta, \delta]} l_{t}^{j}(s) d s$. Therefore, for $n>\max \left\{n_{0}, n_{1}\right\}$, from the two previous inequalities we obtain a contradiction which implies the uniform limit in (5.19).

In order to conclude the proof, consider $\left(f_{n}\right)_{n \in \mathbb{N}} \subset \mathfrak{L} \mathfrak{C}\left(\mathbb{R}^{2 N}, \mathbb{R}^{N}\right)$ converging to some $f$ in $\left(\mathfrak{L C}\left(\mathbb{R}^{2 N}, \mathbb{R}^{N}\right), \mathcal{T}_{\Theta} \widehat{\Theta}\right)$ and $\left(t_{n}\right)_{n \in \mathbb{N}} \subset \mathbb{R}$ converging to some $t \in \mathbb{R}$. Fixed $j \in \mathbb{N}, I=\left[q_{1}, q_{2}\right]$ with $q_{1}, q_{2} \in \mathbb{Q}$, and $\mathcal{K}_{j}^{I}$ and $\widehat{\mathcal{K}}_{j}^{I-1}$ as in Definition 5.4, recalling that the limit in (5.18) is uniform for $t$ in compact intervals, we have that

$$
\begin{aligned}
& \lim _{n \rightarrow \infty} \sup _{x(\cdot) \in \mathcal{K}_{j}^{I}, u(\cdot) \in \widehat{\mathcal{K}}_{j}^{I-1}} \int_{I}\left|\left(f_{n}\right)_{t_{n}}(s, x(s), u(s-1))-f_{t}(s, x(s), u(s-1))\right| d s \\
& \leq \lim _{n \rightarrow \infty} \sup _{x(\cdot) \in \mathcal{K}_{j}^{I}, u(\cdot) \in \widehat{\mathcal{K}}_{j}^{I-1}} \int_{I}\left|\left(f_{n}\right)_{t_{n}}(s, x(s), u(s-1))-f_{t_{n}}(s, x(s), u(s-1))\right| d s \\
& \quad+\lim _{n \rightarrow \infty} \sup _{x(\cdot) \in \mathcal{K}_{j}^{I}, u(\cdot) \in \widehat{\mathcal{K}}_{j}^{I-1}} \int_{I}\left|f_{t_{n}}(s, x(s), u(s-1))-f_{t}(s, x(s), u(s-1))\right| d s=0,
\end{aligned}
$$

which ends the proof for the topology $\mathcal{T}_{\Theta} \widehat{\Theta}$.

The proof for the topology $\mathcal{T}_{\Theta D}$ can be easily deduced from the previous one and thus we skip it. As regard the topology $\mathcal{T}_{\Theta B}$, notice that we can still use the same arguments till formula (5.22) except fo the fact that now the sequence $\left(u_{n}(\cdot)\right)_{n \in \mathbb{N}}$ is only bounded, that is for all $n \in \mathbb{N}$ one has $u_{n}(\cdot) \in C\left(I-1, B_{j}\right)$, and thus it does not necessarily admit a converging subsequence. Therefore, we change the proof as follows. Denote by $l_{1}^{2 j}(\cdot) \in L_{l o c}^{1}$ the optimal $l_{1}$-bound for the function $f$ on $B_{2 j} \subset \mathbb{R}^{2 N}$. Then we can write

$$
\begin{aligned}
\varepsilon<\left\|f_{t+\tau_{n}}\left(\cdot, x_{n}(\cdot), u_{n}(\cdot-1)\right)-f_{t}\left(\cdot, x_{n}(\cdot), u_{n}(\cdot-1)\right)\right\|_{L^{1}(I)} \\
\leq\left\|f_{t+\tau_{n}}\left(\cdot, x_{n}(\cdot), u_{n}(\cdot-1)\right)-f_{t+\tau_{n}}\left(\cdot, x(\cdot), u_{n}(\cdot-1)\right)\right\|_{L^{1}(I)} \\
\quad+\left\|f_{t+\tau_{n}}\left(\cdot, x(\cdot), u_{n}(\cdot-1)\right)-f_{t}\left(\cdot, x(\cdot), u_{n}(\cdot-1)\right)\right\|_{L^{1}(I)} \\
\quad+\left\|f_{t}\left(\cdot, x(\cdot), u_{n}(\cdot-1)\right)-f_{t}\left(\cdot, x_{n}(\cdot), u_{n}(\cdot-1)\right)\right\|_{L^{1}(I)} \\
\leq\left\|x_{n}(\cdot)-x(\cdot)\right\|_{L^{\infty}(I)}\left[\int_{I}\left(l_{1}^{2 j}\right)_{t+\tau_{n}}(s) d s+\int_{I}\left(l_{1}^{2 j}\right)_{t}(s) d s\right] \\
\quad+\left\|f_{t+\tau_{n}}\left(\cdot, x(\cdot), u_{n}(\cdot-1)\right)-f_{t}\left(\cdot, x(\cdot), u_{n}(\cdot-1)\right)\right\|_{L^{1}(I)}
\end{aligned}
$$

Let us analyze separately the two elements of the sum on the right-hand side of the previous chain of inequalities. As regard the first one, recall that we can consider $\delta>0$ such that $\left|\tau_{n}\right|<\delta$ for all $n \in \mathbb{N}$, and thus write

$$
\begin{aligned}
& \left\|x_{n}(\cdot)-x(\cdot)\right\|_{L^{\infty}(I)}\left[\int_{I}\left(l_{1}^{2 j}\right)_{t+\tau_{n}}(s) d s+\int_{I}\left(l_{1}^{2 j}\right)_{t}(s) d s\right] \\
& \leq 2\left\|x_{n}(\cdot)-x(\cdot)\right\|_{L^{\infty}(I)} \int_{I+[-\delta, \delta]}\left(l_{1}^{2 j}\right)_{t}(s) d s<\varepsilon / 2,
\end{aligned}
$$


where the last inequality is true recalling that $\left(x_{n}(\cdot)\right)_{n \in \mathbb{N}}$ converges uniformly to $x(\cdot) \in \mathcal{K}_{j}^{I}$ and thus there exists $n_{0} \in \mathbb{N}$ such that for $n>n_{0}$ one has

$$
\left\|x_{n}(\cdot)-x(\cdot)\right\|_{L^{\infty}(I)}<\frac{\varepsilon}{2 C}, \quad \text { where } C=2 \int_{I+[-\delta, \delta]}\left(l_{1}^{2 j}\right)_{t}(s) d s .
$$

Furthermore, as regard the second element of the sum on the right-hand side of (5.24), one has

$$
\begin{aligned}
& \left\|f_{t+\tau_{n}}\left(\cdot, x(\cdot), u_{n}(\cdot-1)\right)-f_{t}\left(\cdot, x(\cdot), u_{n}(\cdot-1)\right)\right\|_{L^{1}(I)} \\
& \leq\left\|f_{t+\tau_{n}}\left(\cdot, x(\cdot), u_{n}(\cdot-1)\right)-f_{t+\tau_{n}}\left(\cdot, x\left(\cdot+\tau_{n}\right), u_{n}(\cdot-1)\right)\right\|_{L^{1}(I)} \\
& \quad+\left\|f_{t+\tau_{n}}\left(\cdot, x\left(\cdot+\tau_{n}\right), u_{n}(\cdot-1)\right)-f_{t}\left(\cdot, x(\cdot), u_{n}(\cdot-1)\right)\right\|_{L^{1}(I)} \\
& \leq\left\|x(\cdot)-x\left(\cdot+\tau_{n}\right)\right\|_{L^{\infty}(I)} \int_{I+[-\delta, \delta]}\left(l_{1}^{2 j}\right)_{t}(s) d s \\
& \quad+\left\|f_{t+\tau_{n}}\left(\cdot, x\left(\cdot+\tau_{n}\right), u_{n}(\cdot-1)\right)-f_{t}\left(\cdot, x(\cdot), u_{n}(\cdot-1)\right)\right\|_{L^{1}(I)} \cdot
\end{aligned}
$$

Since $\left(\tau_{n}\right)_{n \in \mathbb{N}}$ in $\mathbb{R}$ converges to 0 , there exists $n_{1} \in \mathbb{N}$ such that for $n>n_{1}$ one has

$$
\left\|x(\cdot)-x\left(\cdot+\tau_{n}\right)\right\|_{L^{\infty}(I)}<\frac{\varepsilon}{4 C},
$$

where, as before, $C=2 \int_{I+[-\delta, \delta]}\left(l_{1}^{2 j}\right)_{t}(s) d s$. Moreover, define $\tilde{f} \in \mathfrak{L} \mathfrak{C}\left(\mathbb{R}^{N}, \mathbb{R}^{N}\right)$ by

$$
\widetilde{f}(s, u)=f(s, x(s), u) .
$$

Therefore, by the continuity of the time translation for $\left(\mathfrak{L} \mathfrak{C}\left(\mathbb{R}^{N}, \mathbb{R}^{N}\right), \mathcal{T}_{B}\right)$ given in [41, Lemma II.1, p.24] one has that

$$
\tilde{f}_{t+\tau_{n}} \stackrel{n \rightarrow \infty}{\longrightarrow} \widetilde{f}_{t} \quad \text { with respect to the topology } \mathcal{T}_{B} .
$$

In particular, considering an interval $J \subset \mathbb{R}$ with extrema in $\mathbb{Q}$ such that $J$ contains the intervals $I, I-1$, and $I+[-\delta, \delta]$ and up to extending the functions in $\mathcal{K}_{j}^{I}$ and $\widehat{\mathcal{K}}_{j}^{I-1}$ by constants to the interval $J$, one has that there exists $n_{2} \in \mathbb{N}$ such that for all $n \geq n_{2}$ one has

$$
\begin{aligned}
\| f_{t+\tau_{n}}(\cdot, & \left.x\left(\cdot+\tau_{n}\right), u_{n}(\cdot-1)\right)-f_{t}\left(\cdot, x(\cdot), u_{n}(\cdot-1)\right) \|_{L^{1}(I)} \\
& \leq \sup _{u(\cdot) \in C\left(J, B_{j}\right)}\left\|f_{t}\left(\cdot+\tau_{n}, x\left(\cdot+\tau_{n}\right), u(\cdot)\right)-f_{t}(\cdot, x(\cdot), u(\cdot))\right\|_{L^{1}(J)} \\
& =\sup _{u(\cdot) \in C\left(J, B_{j}\right)}\left\|\tilde{f}_{t+\tau_{n}}(\cdot, u(\cdot))-\tilde{f}_{t}(\cdot, u(\cdot))\right\|_{L^{1}(J)}<\varepsilon / 4 .
\end{aligned}
$$

Therefore, gathering together (5.24), (5.25), (5.26), (5.27) and (5.28) one obtains the following contradiction

$$
\varepsilon<\left\|f_{t+\tau_{n}}\left(\cdot, x_{n}(\cdot), u_{n}(\cdot-1)\right)-f_{t}\left(\cdot, x_{n}(\cdot), u_{n}(\cdot-1)\right)\right\|_{L^{1}(I)}<\varepsilon .
$$

Consequently, we obtain the uniform limit in (5.19). The joint continuity for the two variables is then proved exactly as in the case of the topology $\mathcal{T}_{\Theta} \widehat{\Theta}$.

The proof for the weak hybrid topologies $\sigma_{\Theta} \widehat{\Theta}$ and $\sigma_{\Theta D}$ can be deduced from the one of Theorem 2.43 with minor modifications. 
Theorem 5.15. Consider $E \in \mathfrak{L} \mathfrak{C}\left(\mathbb{R}^{2 N}, \mathbb{R}^{N}\right)$ with $L_{\text {loc }}^{1}$-equicontinuous $m$-bounds and let $\Theta$ be the suitable set of moduli of continuity given by the $m$-bounds as in Definition 3.2. Moreover, let $D$ be any dense and countable subset of $\mathbb{R}^{N}$. With the notation of Theorem 5.11, the following statements hold:

(i) if $\left(f_{n}\right)_{n \in \mathbb{N}}$ is a sequence in $E$ converging to $f$ in $\left(\mathfrak{L} \mathfrak{C}\left(\mathbb{R}^{2 N}, \mathbb{R}^{N}\right), \mathcal{T}_{\Theta B}\right)$ and $\left(\phi_{n}(\cdot)\right)_{n \in \mathbb{N}}$ is a sequence in $\mathcal{C}$ converging uniformly to some function $\phi \in \mathcal{C}$, then

$$
x\left(\cdot, f_{n}, \phi_{n}\right) \stackrel{n \rightarrow \infty}{\longrightarrow} x(\cdot, f, \phi)
$$

uniformly in any $[-1, T] \subset I_{f, \phi}$;

(ii) if $E$ has also $L_{\text {loc }}^{1}$-bounded $l_{2}$-bounds, $\left(f_{n}\right)_{n \in \mathbb{N}}$ is a sequence in $E$ converging to $f$ in $\left(\mathfrak{L C}\left(\mathbb{R}^{2 N}, \mathbb{R}^{N}\right), \mathcal{T}_{\Theta D}\right)$ and $\left(\phi_{n}(\cdot)\right)_{n \in \mathbb{N}}$ is a sequence in $\mathcal{C}$ converging uniformly to $\phi \in \mathcal{C}$, then

$$
x\left(\cdot, f_{n}, \phi_{n}\right) \stackrel{n \rightarrow \infty}{\longrightarrow} x(\cdot, f, \phi)
$$

uniformly in any $[-1, T] \subset I_{f, \phi}$;

(iii) if $E$ has also $L_{\text {loc }}^{1}$-bounded $l_{2}$-bounds, $\left(f_{n}\right)_{n \in \mathbb{N}}$ is a sequence in $E$ converging to $f$ in $\left(\mathfrak{L} \mathfrak{C}\left(\mathbb{R}^{2 N}, \mathbb{R}^{N}\right), \sigma_{\Theta D}\right)$ and $\left(\phi_{n}(\cdot)\right)_{n \in \mathbb{N}}$ is a sequence in $\mathcal{C}$ converging uniformly to $\phi \in \mathcal{C}$, then

$$
x\left(\cdot, f_{n}, \phi_{n}\right) \stackrel{n \rightarrow \infty}{\longrightarrow} x(\cdot, f, \phi)
$$

uniformly in any $[-1, T] \subset I_{f, \phi}$;

Proof. Firstly, notice that once we prove (i), we immediately obtain (ii) thanks to Theorem 5.9.

As regards both (i) and (iii), proceed as in the proof of Theorem 5.11 till Case 1 included. Case 2 has to be treated differently, yet notice that in both the cases, for each $n \in \mathbb{N}$, denoted by $m_{n}^{2 j}(\cdot)$ the optimal $m$-bound for $f_{n}$ on $B_{2 j} \subset \mathbb{R}^{2 N}$, one can write

$$
\begin{aligned}
\left|z_{n}\left(t_{1}\right)-z_{n}\left(t_{2}\right)\right| & \leq\left|\int_{t_{1}}^{t_{2}} f_{n}\left(s, z_{n}(s), z_{n}(s-1)\right) d s\right| \\
& \leq \int_{t_{1}}^{t_{2}}\left|f_{n}\left(s, z_{n}(s), z_{n}(s-1)\right)\right| d s \leq \int_{t_{1}}^{t_{2}} m_{n}^{j}(s) d s \\
& \leq \sup _{n \in \mathbb{N}} \int_{t_{1}}^{t_{2}} m_{n}^{j}(s) d s
\end{aligned}
$$

and, due to the $L_{l o c}^{1}$-equicontinuity of the $m$-bounds we obtain the result. The rest of the proof is as for Theorem 5.11 with the exception that in order to pass from (5.15) to (5.17) as $n \rightarrow \infty$, the limit has to be taken in the proper topology, either $\mathcal{T}_{\Theta B}$ for (i), or $\sigma_{\Theta D}$ for (iii). We shall treat the two cases separately and with further details. 
(i) In order to pass from (5.15) to (5.17) as $n \rightarrow \infty$, we use the topology $\mathcal{T}_{\Theta B}$. Notice that everything is consistent since $z_{n}(t)=x\left(t, f_{n}, \phi_{n}\right)$ for any $t \in$ $\left[-1, T_{0}\right]$ and $n>n_{0}$, and $\left(z_{n}(\cdot)\right)_{n \in \mathbb{N}}$ is uniformly bounded in $\left[-1, T_{0}\right]$ by construction. Therefore, instead of $(5.16)$, for all $t \in\left[0, T_{0}\right] \cap \mathbb{Q}$, we can write

$$
\begin{gathered}
\left|\int_{0}^{t} f_{n}\left(s, z_{n}(s), z_{n}(s-1)\right) d s-\int_{0}^{t} f(s, z(s), z(s-1)) d s\right| \\
\leq \sup _{\substack{x(\cdot) \in \mathcal{K}_{j}^{[0, t]} \\
u(\cdot) \in C\left([-1, t-1], B_{j}\right)}} \int_{0}^{t}\left|f_{n}(s, x(s), u(s-1))-f(s, x(s), u(s-1))\right| d s \\
+2\left\|z_{n}(\cdot)-z(\cdot)\right\|_{L^{\infty}([-1, \beta])} \int_{0}^{t} l_{f}^{2 j}(s) d s .
\end{gathered}
$$

As said, the rest of the proof is as for Theorem 5.11.

(iii) In order to pass from (5.15) to (5.17) as $n \rightarrow \infty$, we should use the topology $\sigma_{\Theta D}$. However, this does not allow to directly obtain the result as in case (i). Yet, thanks to Theorem 5.9 we know that $\sigma_{\Theta D}$ coincides with any topology of the type $\sigma_{\Theta} \widehat{\Theta}$, where $\widehat{\Theta}$ is a suitable set of moduli of continuity. In particular, since $\left(\phi_{n}(\cdot)\right)_{n \in \mathbb{N}}$ converges uniformly to $\phi \in \mathcal{C}$, then by Ascoli-Arzelá's theorem, there exists a shared modulus of continuity $\theta_{0} \in C\left(\mathbb{R}^{+}, \mathbb{R}^{+}\right)$. Let us consider the following suitable set of moduli of continuity

$$
\widehat{\Theta}:=\left\{\hat{\theta}_{j}^{I}(\cdot) \in C\left(\mathbb{R}^{+}, \mathbb{R}^{+}\right) \mid \hat{\theta}_{j}^{I}(s)=\max \left\{\theta_{0}(s), \theta_{j}^{I}(s)\right\}\right\}
$$

Then, as said, since $\left(f_{n}\right)_{n \in \mathbb{N}}$ converges to $f$ with respect to $\sigma_{\Theta D}$, then it also converges to $f$ with respect to $\sigma_{\Theta} \widehat{\Theta}$. Therefore, instead of (5.16), for all $t \in[0, \beta] \cap \mathbb{Q}$, we can now write

$$
\begin{aligned}
& \left|\int_{0}^{t} f_{n}\left(s, z_{n}(s), z_{n}(s-1)\right) d s-\int_{0}^{t} f(s, z(s), z(s-1)) d s\right| \\
& \leq \sup _{x(\cdot) \in \mathcal{K}_{j}^{[0, t]}, u(\cdot) \in \widehat{\mathcal{K}}_{j}^{[-1, t-1]}}\left|\int_{0}^{t}\left[f_{n}(s, x(s), u(s-1))-f(s, x(s), u(s-1))\right] d s\right| \\
& \quad+2\left\|z_{n}(\cdot)-z(\cdot)\right\|_{L^{\infty}([-1, \beta])} \int_{0}^{t} l_{f}^{2 j}(s) d s .
\end{aligned}
$$

As said, the rest of the proof is, in both the cases, as for Theorem 5.11.

As a consequence of the previous theorem, one can obtain a new theorem of continuity of the induced skew product semiflow for the topologies $\mathcal{T}_{\Theta B}, \mathcal{T}_{\Theta D}$ and $\sigma_{\Theta D}$, with $\Theta$ being a suitable set of moduli of continuity (see Definition 2.3) and $D$ a dense and countable subset of $\mathbb{R}^{N}$.

Consider $f \in \mathfrak{L} \mathfrak{C}\left(\mathbb{R}^{2 N}, \mathbb{R}^{N}\right)$ with $L_{\text {loc }}^{1}$-equicontinuous $m$-bounds, a dense and countable set $D \subset \mathbb{R}^{N}$ and let $\Theta$ be defined as in Definition 3.2. With the 
notation introduced in Theorem 5.1, let us denote by $\mathcal{U}_{\mathcal{T}_{\Theta B}}$ the subset of $\mathbb{R} \times$ $\operatorname{Hull}_{\left(\mathfrak{L C}, \mathcal{T}_{\Theta B}\right)}(f) \times \mathcal{C}$ given by

$$
\mathcal{U}_{\mathcal{T}_{\Theta B}}=\bigcup_{\substack{g \in \operatorname{Hull}_{\left(\mathfrak{S c}, \mathcal{T}_{\Theta B}\right)}(f) \\ \phi \in \mathcal{C}}}\left\{(t, g, \phi) \mid t \in I_{g, \phi}\right\}
$$

by $\mathcal{U}_{\mathcal{T}_{\Theta D}}$ the subset of $\mathbb{R} \times \operatorname{Hull}_{\left(\mathfrak{L C}, \mathcal{T}_{\Theta D}\right)}(f) \times \mathcal{C}$ given by

$$
\mathcal{U}_{\mathcal{T}_{\Theta D}}=\bigcup_{\substack{g \in \operatorname{Hull}_{\left(\mathfrak{S c}, \mathcal{T}_{\Theta D}\right)}(f) \\ \phi \in \mathcal{C}}}\left\{(t, g, \phi) \mid t \in I_{g, \phi}\right\} .
$$

and by $\mathcal{U}_{\sigma_{\Theta D}}$ the subset of $\mathbb{R} \times \operatorname{Hull}_{\left(\mathfrak{L C}, \mathcal{T}_{\Theta D}\right)}(f) \times \mathcal{C}$ given by

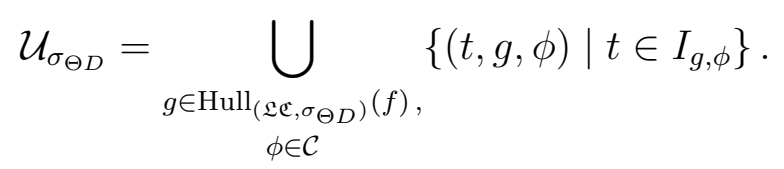

Theorem 5.16. Consider $f \in \mathfrak{L} \mathfrak{C}\left(\mathbb{R}^{2 N}, \mathbb{R}^{N}\right)$ with $L_{\text {loc }}^{1}$-equicontinuous $m$-bounds and let $\Theta$ be defined as in Definition 3.2.

(i) The set $\mathcal{U}_{\mathcal{T}_{\Theta B}}$ is open in $\mathbb{R} \times \operatorname{Hull}_{\left(\mathfrak{L C}, \mathcal{T}_{\Theta B}\right)}(f) \times \mathcal{C}$ and the map

$$
\begin{aligned}
\mathcal{U}_{\mathcal{T}_{\Theta B}} \subset \mathbb{R} \times \operatorname{Hull}_{\left(\mathfrak{L C}, \mathcal{T}_{\Theta B}\right)}(f) \times \mathcal{C} & \rightarrow \operatorname{Hull}_{\left(\mathfrak{L C} \mathfrak{C}, \mathcal{T}_{\Theta B}\right)}(f) \times \mathcal{C} \\
(t, g, \phi) & \mapsto \quad\left(g_{t}, x_{t}(\cdot, g, \phi)\right)
\end{aligned}
$$

defines a local continuous skew-product semiflow on $\operatorname{Hull}_{\left(\mathfrak{L C}, \mathcal{T}_{\Theta B}\right)}(f) \times \mathcal{C}$.

If additionally $f$ has $L_{\text {loc-bounded }}^{1} l_{2}$-bounds, then the following statements hold true.

(ii) The set $\mathcal{U}_{\mathcal{T}_{\Theta D}}$ is open in $\mathbb{R} \times \operatorname{Hull}_{\left(\mathfrak{L C}, \mathcal{T}_{\Theta D}\right)}(f) \times \mathcal{C}$ and the map

$$
\begin{aligned}
\mathcal{U}_{\mathcal{T}_{\Theta D}} \subset \mathbb{R} \times \operatorname{Hull}_{\left(\mathfrak{L C}, \mathcal{T}_{\Theta D}\right)}(f) \times \mathcal{C} & \rightarrow \operatorname{Hull}_{\left(\mathfrak{L C}, \mathcal{T}_{\Theta D}\right)}(f) \times \mathcal{C} \\
(t, g, \phi) & \mapsto \quad\left(g_{t}, x_{t}(\cdot, g, \phi)\right)
\end{aligned}
$$

defines a local continuous skew-product semiflow on $\operatorname{Hull}_{\left(\mathfrak{L C}, \mathcal{T}_{\Theta D}\right)}(f) \times \mathcal{C}$.

(iii) The set $\mathcal{U}_{\sigma_{\Theta D}}$ is open in $\mathbb{R} \times \operatorname{Hull}_{\left(\mathfrak{L C}, \sigma_{\Theta D}\right)}(f) \times \mathcal{C}$ and the map

$$
\begin{aligned}
\mathcal{U}_{\sigma_{\Theta D}} \subset \mathbb{R} \times \operatorname{Hull}_{\left(\mathfrak{L C}, \sigma_{\Theta D}\right)}(f) \times \mathcal{C} & \rightarrow \operatorname{Hull}_{\left(\mathfrak{L C}, \sigma_{\Theta D}\right)}(f) \times \mathcal{C} \\
(t, g, \phi) & \mapsto \quad\left(g_{t}, x_{t}(\cdot, g, \phi)\right)
\end{aligned}
$$

defines a local continuous skew-product semiflow on $\operatorname{Hull}_{\left(\mathfrak{L C}, \sigma_{\Theta D}\right)}(f) \times \mathcal{C}$. 


\section{Conclusions}

The theory developed in this work allows to extend the skew-product formalism to Carathéodory ordinary differential equations and delay differential equations with constant delay through the use of strong and weak metric topologies of integral type. As a result, one obtains a variety of tools from topological dynamics to study the qualitative behavior of the solutions of such classes of differential problems.

As an example, the work includes several applications for Carathéodory ODEs such as linearized skew-product flows, propagation of the exponential dichotomy and of the dichotomy spectrum of a linear system and study of pullback and global attractors, as well as some simple motivational examples taken from modelizations of real phenomena, which aim to show the applicability of the theory. Additionally, the thesis provides a rich description of the topological structure of the considered spaces of Carathéodory functions (among which, some are new) presenting, for example, characterizations of the classes of equivalences for functions which differ on negligible subset of $\mathbb{R} \times \mathbb{R}^{N}$, propagation of properties on the so-called $m$-bounds and $l$-bounds through the limits in the given topologies, and sufficient conditions of relative compactness for subsets of Lipschitz Carathéodory functions.

Having constructed several different skew-product flows also for Carathéodory DDEs with constant delay, it seems natural that many of the previously cited results could be possibly developed also for this class of differential problems and thus we aim to include them in future publications. Furthermore, the theory hereby developed offers many inspirations for a further investigation for both the classes of problems. Among the many possible research directions for the future, we like to mention applications in bifurcation theory, control theory, mathematical biology and numerical analysis. 



\section{Summary in Spanish}

Un ejercicio elemental de análisis matemático consiste en probar que, para cada función continua $f: D \subset \mathbb{R} \times \mathbb{R}^{N} \rightarrow \mathbb{R}^{N}$, el problema de Cauchy

$$
\dot{x}=f(t, x), \quad x\left(t_{0}\right)=x_{0},
$$

es equivalente al problema integral

$$
x(t)=x_{0}+\int_{t_{0}}^{t} f(s, x(s)) d s .
$$

Sin embargo, gracias a la teoría de integración de Lebesgue, es fácil darse cuenta de que la fórmula (S.2) tiene sentido para una clase de funciones más amplia que la de las continuas. Es decir, dado un intervalo $I \subset \mathbb{R}$ de manera que $t_{0} \in I$, si uno busca una función $x: I \subset \mathbb{R} \rightarrow \mathbb{R}^{N}$ que no sea necesariamente derivable y satisfaga (S.2) en su intervalo de definición, entonces se pueden relajar las hipótesis sobre $f$ a la integrabilidad local. El matemático griego Constantin Carathéodory probó en [15] que, bajo las hipótesis,

- $f$ medible Borel,

- para cada conjunto compacto $K \subset \mathbb{R}^{N}$ existe una función real $m^{K} \in L_{l o c}^{1}$ tal que para casi todo $t \in \mathbb{R}$, se tiene

$$
|f(t, x)| \leq m^{K}(t) \quad \text { para todo } x \in K
$$

- para casi todo $t \in \mathbb{R}, f(t, \cdot)$ es continua,

cualquier problema de tipo (S.1) admite una solución generalizada, es decir, una función absolutamente continua, definida sobre un intervalo $I \subset \mathbb{R}$ que contiene $t_{0}$, tal que (S.1) se satisface en casi todo punto de $I$ o, equivalentemente, (S.2) se verifica para todo punto de $I$.

Esa clase de problemas diferenciales toma su nombre de Carathéodory y ha contribuido al enriquecimiento del campo de aplicación de las ecuaciones diferenciales tanto en los estudios teóricos como en los prácticos. De hecho, el desarrollo de una teoría matemática consistente para ecuaciones diferenciales con campo vectorial discontinuo ha sido, de alguna manera, impulsada por muchas de sus 
aplicaciones a problemas en teoría de control, en mecánica y en ingeniería eléctrica, donde el uso de interruptores, relés y señales digitales, no sólo es habitual, sino incluso necesario para conseguir resultados óptimos (véase Bressan y Piccoli [11], Brogliato [12], Clarke [19]).

El objetivo de este trabajo es aplicar herramientas de dinámica no autónoma a ecuaciones diferenciales de Carathéodory y, en particular, definir un flujo triangular continuo que permita el estudio cualitativo de las soluciones. La noción de flujo triangular se debe a Bebutov [7] y se ha convertido en una herramienta fundamental en dinámica no autónoma. Para poder recuperar una estructura de grupo en la evolución de un sistema no-autónomo (lo que es inmediato para sistemas dinámicos autónomos, donde se puede construir un flujo a través de las soluciones), la idea es considerar simultáneamente la solución y la evolución del campo vectorial en el tiempo. Si las dos componentes son continuas respecto del tiempo, del campo vectorial inicial y de los datos iniciales, entonces la aplicación así obtenida, define un flujo triangular continuo.

El estudio de las topologías de continuidad para flujos triangulares generados por ecuaciones diferenciales de Carathéodory es un tema clásico que fue iniciado por Miller y Sell [41, 42] y posteriormente tratado por Artstein [3, 4, 5], Heunis [30], Neustadt [43], Opial [45], Sell [52, 53], entre otros muchos autores. Desde entonces, se han utilizado topologías fuertes y débiles de tipo integral para investigar ecuaciones diferenciales no autónomas lineales (véase Bodin y Sacker [10], Chow y Leiva [20] y Siegmund [55] entre otros) y, sin embargo, a pesar de su interés potencial, la teoría clásica no ha sido convenientemente desarrollada en el caso no lineal.

En este trabajo armonizamos muchos de los resultados obtenidos en Longo et al. $[38,39]$ y continuamos con el estudio para completar la teoría original, mejorar su aplicabilidad y realizar un análisis exhaustivo del comportamiento cualitativo de las soluciones. En particular, definimos nuevas topologías métricas en espacios vectoriales localmente convexos adecuados, en los que la aplicación de translación en el tiempo resulta continua, así como las soluciones varían continuamente respecto de los datos iniciales. De esa forma, en dichos espacios conseguimos un flujo triangular continuo formado por el flujo base de las trasladadas en el tiempo sobre la envolvente de un campo vectorial y por las soluciones del respectivo problema diferencial.

En la demostración de estos resultados juega un papel importante el estudio meticuloso de las $m$-cotas y $l$-cotas de una función de Carathéodory $f$, es decir, las familias de funciones positivas y localmente integrables que sirven, respectivamente, como acotación para el módulo de $f$ y como coeficiente de Lipschitz para $f$ sobre los compactos de $\mathbb{R}^{N}$. Además, el análisis de las $m$-cotas y l-cotas nos permite obtener alguna información topológica adicional. En particular, caracterizamos los subconjuntos relativamente compactos de funciones Lipschitz Carathéodory y aclaramos las condiciones (de hecho bastante débiles) 
bajo las cuales las topologías (fuertes o débiles) consideradas en nuestro trabajo, así como anteriormente en la literatura, coinciden. Esos resultados son particularmente importantes, no sólo porque contribuyen a homogeneizar toda la teoría, sino porque, en los casos en los que se puedan aplicar, mejoran o simplifican las herramientas matemáticas disponibles. En concreto, en el primer caso podemos obtener la existencia de una medida ergódica invariante para el flujo en la base y la posibilidad de utilizar técnicas de teoría ergódica, mientras que en el segundo caso, podemos elegir, entre las posibles topologías, las más fáciles de manejar como las que suponen una especie de convergencia puntual. Curiosamente, muchas de las aplicaciones en ingeniería y ciencias aplicadas se basan en hipótesis que son más fuertes de las que se necesitan para aplicar estos resultados.

Completamos la importancia de los resultados de continuidad obtenidos para flujos triangulares con algunas aplicaciones teóricas. En particular, definimos dos tipos de flujos linealizados y obtenemos la derivabilidad con respecto a las condiciones iniciales de las soluciones de algunos sistemas que no son derivables en la variable $x$ y por tanto, no tienen una ecuación variacional clásica. Además, propagamos la dicotomía exponencial de un sistema lineal, así como su espectro de Sacker-Sell, a través de las trayectorias de estos flujos linealizados. Finalmente, incluimos también un resultado de existencia de soluciones para algunas ecuaciones de Carathéodory cuyo campo vectorial es posiblemente discontinuo en la variable $x$ y por tanto, no se le puede aplicar el teorema de Carathéodory. La prueba es independiente de la teoría de Filippov y se basa en la teoría de Carathódory y el teorema de continuidad de las soluciones con respecto al campo vectorial.

Como consecuencia de estos resultados, se abre una gama de escenarios dinámicos en los que es posible combinar técnicas de flujos triangulares continuos, procesos, y sistemas dinámicos aleatorios (véase Arnold [2], Aulbach y Wanner [6], Berger y Siegmund [9], Caraballo y Han [13], Carvalho et al. [16], Johnson et al. [34], Kloeden y Rasmussen [36], Sell [53], Shen y Yi [54] y las referencias citadas en ellos). En concreto, combinando cuidadosamente el uso simultáneo de los formalismos de procesos y flujos triangulares, somos capaces de probar la existencia de determinados conjuntos acotados y absorbentes para el proceso inducido por un campo vectorial $f$ en una clase específica de ecuaciones de Carathéodory. Esto nos permite encontrar atractores pullback acotados para los procesos inducidos por ecuaciones cuyos campos vectoriales están en el conjunto alpha-límite, en el conjunto omega-límite o en toda la envolvente de $f$. Bajo condiciones adecuadas, probamos además la existencia de un atractor pullback o de un atractor global para el respectivo flujo triangular.

Como última contribución teórica, queremos dejar la teoría preparada para que los resultados obtenidos en este trabajo se puedan generalizar a ecuaciones diferenciales de Carathéodory con retardo del tipo

$$
\dot{x}=f(t, x(t), x(t-\tau)) \text {. }
$$


Ese problema tiene la dificultad intrínseca añadida de ser infinito dimensional debido al hecho de que el espacio fase es el espacio de las funciones continuas definidas en el intervalo $[-\tau, 0]$. Sin embargo, el tipo de ecuación en concreto y el hecho que $f$ esté definida en un espacio finito dimensional, nos permite utilizar algunas de las técnicas y de los razonamientos citados anteriormente, para extender, hasta un cierto punto y con las inevitables discrepancias, la teoría desarrollada para ecuaciones ordinarias de Carathéodory a esta clase de problemas. En particular, construiremos un semiflujo triangular continuo asociado a (S.3), respecto de varias topologías métricas (débiles y fuertes). Es interesante notar que, a pesar de ser una clase muy específica de problemas diferenciales, las ecuaciones como (S.3) están ampliamente utilizadas in ingeniería y ciencias aplicadas para modelizar muchos fenómenos reales en los que el pasado afecta el futuro.

A lo largo de la memoria, aplicaremos algunos de los resultados obtenidos a tres ejemplos que han sido sacado de unos modelos concretos de mecánica (K. Popp and P. Stelter [47]), teoría de control (Fabbri et al. [25]) y biología matemática (Rasmussen et al. [50]). No pretendemos hacer una exposición exhaustiva, sino demostrar la vasta aplicabilidad de los resultados obtenidos en este trabajo.

La teoría de ecuaciones diferenciales de Carathéodory contiene, a día de hoy, casi un siglo de resultados matemáticos. Desde el punto de vista de los sistemas dinámicos se han realizado muchos estudios en estabilidad, análisis númerico y teoría de bifurcación (véase, por ejemplo, Filippov [26], Osinenko et al [46], Pötzsche y Rasmussen [49], Pötszche [48], entre otros). Los resultados obtenidos en esta memoria pueden ser aplicados en estas direcciones.

A continuación proporcionamos un resumen un poco más detallado de cada uno de los capítulos contenidos en la memoria.

\section{Preliminares}

En este capítulo, fijamos la notación y proporcionamos algunas nociones básicas que sirven de preliminares para el contenido de la memoria. El capítulo está dividido en dos secciones. En la sección 1.1 se recuerdan definiciones y resultados clásicos de ecuaciones diferenciales ordinarias de Carathéodory como el teorema de existencia y unicidad de soluciones para un problema de Cauchy y el teorema de variación continua respecto de los datos iniciales. Además, presentamos un ejemplo de un fenómeno de vibración mediante la modelización de una cuerda de violín utilizando condiciones de Carathéodory.

La sección 1.2 contiene nociones elementales de dinámica no autónoma como las definiciones de proceso y flujo triangular y la manera en la que una ecuación diferencial puede inducir cada uno de ellos. Además, se dan las definiciones de envolvente de una función, de dicotomía exponencial y de espectro dicotómico. 


\section{Espacios y Topologías}

Este capítulo contiene el marco topológico en el que se basan la mayor parte de los resultados de los capítulos siguientes. En la sección 2.1 presentamos todos los espacios de funciones que se utilizarán para el estudio de las ecuaciones diferenciales ordinarias de Carathéodory. Junto a los espacios clásicos de funciones Lipschitz Carathéodory ( $\mathfrak{L} \mathfrak{C})$ y Strong Carathéodory $(\mathfrak{S} \mathfrak{C})$, introducimos dos espacios nuevos que contienen a los anteriores y cuyos elementos son funciones de Carathéodory que no son necesariamente continuas en la variable $x$ : los espacios de funciones $\Theta$-Carathéodory $(\Theta \mathfrak{C})$ y weak $\Theta$-Carathéodory ( $\mathfrak{W} \Theta \mathfrak{C})$. El símbolo $\Theta$ representa un conjunto de módulos de continuidad que identifican una cantidad numerable de compactos de funciones continuas en los que las funciones de $\Theta \mathfrak{C} \mathrm{y} / \mathrm{o}$ $\mathfrak{W} \Theta \mathfrak{C}$ se comporten "bien", es decir, de (alguna) manera continua en $L_{l o c}^{1}$. Estos espacios jugarán un papel importante en el desarrollo de algunos resultados de esta memoria. Además, tratamos el problema de la identificación de las funciones que difieren en un conjunto de medida cero.

En la sección 2.2 dotamos los espacios anteriores de topologías métricas de tipo integral (débil o fuerte). Entre ellas, se distinguen dos clases nuevas de topologías, las $\mathcal{T}_{\Theta}$ y las $\sigma_{\Theta}$; la convergencia (fuerte o débil, respectivamente) para las sucesiones en $\left(\Theta \mathfrak{C}, \mathcal{T}_{\Theta}\right)$ y en $\left(\mathfrak{W} \Theta \mathfrak{C}, \sigma_{\Theta}\right)$ deberá ser uniforme sobre los compactos de funciones continuas determinados por $\Theta$.

En las secciones siguientes nos centramos en un estudio más cuidadoso de las $m$-cotas y $l$-cotas. El papel de estas funciones, una vez que se les dote de una estructura adecuada, va más allá de la existencia y unicidad proporcionadas por el Teorema 1.2 para las ecuaciones diferenciales de Carathéodory. En particular, en la sección 2.3 introducimos las nociones de $L_{l o c}^{p}$-acotación y $L_{l o c}^{1}$-equicontinuidad que posteriormente se relacionan con los conceptos correspondientes para las funciones de Carathéodory a través de sus $m$-cotas y $l$-cotas.

Como primera aplicación, en la sección 2.4 probamos que la $L_{l o c}^{p}$-acotación y la $L_{l o c}^{1}$-equicontinuidad de las $m$-cotas y las $l$-cotas en conjuntos de funciones de Carathéodory se conserva respecto de la clausura topológica en los casos:

(a) Clausura de subconjuntos en $\mathfrak{S} \mathfrak{C}$ o $\mathfrak{L} \mathfrak{C}$ respecto de cualquiera de las topologías introducidas.

(b) Clausura de subconjuntos en $\mathfrak{S C}_{p}$ o $\mathfrak{L C}_{p}$, con $1<p<\infty$, respecto de cualquiera de las topologías fuertes introducidas.

(c) Clausura de subconjuntos en $\left(\Theta \mathfrak{C}, \mathcal{T}_{\Theta}\right)$ y en $\left(\mathfrak{W} \Theta \mathfrak{C}, \sigma_{\Theta}\right)$.

Esta propagación es crucial porque nos permitirá tratar la clausura de dicho conjuntos como "homogénea" (respecto a una propiedad específica). Citamos dos ejemplos concretos: el límite de una sucesión de funciones convergente, con $l$ cotas $L_{l o c}^{p}$-acotadas, es una función del espacio $\mathfrak{L} \mathfrak{C}$ y por consiguiente, el problema 
diferencial inducido tendrá automáticamente unicidad de soluciones. Por otro lado, si consideramos una sucesión de funciones, con $m$-cotas $L_{l o c}^{1}$-equicontinuas y que sea convergente en $\mathfrak{S} \mathfrak{C}$, entonces las soluciones del problema diferencial inducido por el límite tendrán el mismo módulo de continuidad que las soluciones de los problemas inducidos por los elementos de la sucesión.

En las secciones 2.5 y 2.6 tratamos dos tipos importantes de resultados para el espacio $\mathfrak{L} \mathfrak{C}$ : las condiciones de equivalencia para las topologías fuertes y para las topologías débiles consideradas, así como el estudio de los conjuntos relativamente compactos. En primer lugar demostramos que en un subconjunto de $\mathfrak{L C}_{p}$ (resp. $\mathfrak{L} \mathfrak{C})$ con $l$-cotas $L_{l o c}^{p}$-acotadas (resp. $L_{l o c}^{1}$-acotadas) las topologías fuertes (resp. débiles) coinciden. Además, proporcionamos una caracterización de los conjuntos relativamente compactos para las topologías fuertes, cuando tienen $l$-cotas $L_{l o c}^{p}$-acotadas, mientras que para las topologías débiles recordamos los resultados existentes en [3] y [4].

Concluimos el capítulo con la sección 2.7, donde utilizamos las propiedades de $L_{l o c}^{1}$-equicontinuidad y de $L_{l o c}^{p}$-acotación para las $m$-cotas y/o las $l$-cotas para probar la continuidad de las trasladadas en el tiempo en los espacios $\left(\Theta \mathfrak{C}_{p}\left(\mathbb{R}^{M}\right), \mathcal{T}_{\Theta}\right)$ y $\left(\mathfrak{W} \Theta \mathfrak{C}\left(\mathbb{R}^{M}\right), \sigma_{\Theta}\right)$. Como consecuencia, podemos construir flujos continuos en las envolventes de una función en $\left(\Theta \mathfrak{C}_{p}\left(\mathbb{R}^{M}\right), \mathcal{T}_{\Theta}\right)$ y $\left(\mathfrak{W} \Theta \mathfrak{C}\left(\mathbb{R}^{M}\right), \sigma_{\Theta}\right)$, lo que es el primer paso para poder definir los flujos triangulares continuos inducidos por los respectivos problemas diferenciales, tarea que se realizará en el siguiente capítulo de la memoria.

\section{Continuidad del flujo para ODEs de tipo Carathéodory}

En este capítulo tratamos el problema de definir un flujo triangular continuo inducido por ecuaciones diferenciales ordinarias de Carathéodory del tipo

$$
\dot{x}=f(t, x), \quad x(0)=x_{0},
$$

y sistemas triangulares de ecuaciones de Carathéodory del tipo

$$
\begin{cases}\dot{x}=f(t, x), & x(0)=x_{0}, \\ \dot{y}=F(t, x) y+h(t, x), & y(0)=y_{0},\end{cases}
$$

donde $f$ es una función Lipschitz Carathéodory, mientras $F$ y $h$ pertenecen, respectivamente a $\mathfrak{W} \Theta \mathfrak{C}$ y $\Theta \mathfrak{C}_{p}$ para que los problemas estén bien puestos.

En la sección 3.1 proporcionamos los resultados de continuidad del flujo triangular, óptimos en relación a la topología utilizada y a las hipótesis sobre el campo vectorial inicial. A continuación, damos un esquema de las hipótesis para los tres casos que demostramos (se simplifica la notación omitiendo la dimensión de los espacios de llegada). 
Caso 1: $\quad f \in\left(\mathfrak{L C}, \sigma_{\Theta}\right)$ con $m$-cotas $L_{l o c}^{1}$-equicontinuas,

$F \in\left(\mathfrak{W} \Theta \mathfrak{C}, \sigma_{\Theta}\right)$ con $m$-cotas $L_{l o c}^{1}$-equicontinuas,

$h \in\left(\mathfrak{W} \Theta \mathfrak{C}, \sigma_{\Theta}\right)$ con $m$-cotas $L_{l o c}^{1}$-equicontinuas.

Caso 2: $\quad f \in\left(\mathfrak{L} \mathfrak{C}_{p}, \mathcal{T}_{\Theta}\right)$ con $m$-cotas $L_{l o c}^{1}$-equicontinuas, $F \in\left(\Theta \mathfrak{C}_{p}, \mathcal{T}_{\Theta}\right), h \in\left(\Theta \mathfrak{C}_{p}, \mathcal{T}_{\Theta}\right)$.

Caso 3: $\quad f \in\left(\mathfrak{L} \mathfrak{C}_{p}, \mathcal{T}_{D}\right)$ con $l$-cotas $L_{\text {loc }}^{p}$-acotadas,

$F \in\left(\Theta \mathfrak{C}_{p}, \mathcal{T}_{\Theta}\right), h \in\left(\Theta \mathfrak{C}_{p}, \mathcal{T}_{\Theta}\right)$.

Se puede apreciar que hay un intercambio entre la fuerza de la topología utilizada y las hipótesis sobre las funciones $f, F, \mathrm{y} h$. Es decir, con pocas hipótesis sobre los campos vectoriales, será necesaria una topología más fuerte y viceversa.

Las secciones restantes del capítulo contienen algunas aplicaciones directas de la continuidad del flujo triangular. En particular, en la sección 3.2 tratamos el caso en que la función $f$ es de clase $C^{1}$ en la variable $x$ y la segunda ecuación de (S.5) es la ecuación variacional de la primera, es decir, $F=J_{x} f$ es una función de $\mathfrak{S} \mathfrak{C}$ y $h=0$. Gracias a los resultados de la sección 3.1, podemos construir dos tipos de flujos linealizados (dependiendo de la topología utilizada, $\mathcal{T}_{\Theta}$ o $\sigma_{\Theta}$ ) y probar la derivabilidad de las soluciones respecto de los datos iniciales para problemas cuyo campo vectorial no es de clase $C^{1}$ en la variable $x \mathrm{y}$, por tanto, no admite una ecuación variacional clásica. Concluimos la sección presentando un ejemplo concreto en el que se verifica este fenómeno.

En la sección 3.3 enseñamos como propagar algunas propiedades de estabilidad de un sistema linealizado a través de los flujos linealizados definidos en la sección anterior.

La sección 3.4 contiene otra aplicación teórica y dos ejemplos. La aplicación teórica es un teorema de existencia de soluciones para problemas diferenciales cuyos campos vectoriales son funciones en $\mathfrak{W} \Theta \mathfrak{C}$ que sean límites, en la topología $\sigma_{\Theta}$, de sucesiones en $\mathfrak{S C}$. Dicho teorema se obtiene gracias al resultado de continuidad de las trasladadas en el tiempo (Teorema 2.43), y al teorema de variación continua de las soluciones respecto de los datos iniciales y de los campos vectoriales contenido en la sección 3.1.

Los dos ejemplos tratados al final del capítulo proceden de [25] y de [50], respectivamente. En el primer caso, notamos que las hipótesis consideradas para conseguir algunos resultados de estabilidad para un esquema abstracto de digitización en teoría de control, son tales que el Teorema 2.31 se puede aplicar y entonces la topología de tipo integral considerada en este artículo coincide con cualquiera de las topologías fuertes utilizadas en esta memoria. Además, evidenciamos que algunos resultados incluidos en la misma referencia se pueden obtener de manera más sencilla utilizando la teoría desarrollada en este trabajo.

Por otro lado, inspirados por el segundo artículo, proponemos un sistema triangular compuesto por un sistema compartimental no lineal no autónomo y 
por una ecuación para la edad media asociada. Indicamos bajo que hipótesis se puede construir un flujo triangular continuo utilizando los teoremas probados en la sección 3.1.

\section{Atractores pullback y atractores globales para ODEs de Carathéodory}

Este capítulo contiene resultados de existencia de atractores para una ecuación ordinaria de Carathéodory (cuyas soluciones se suponen definidas hasta $+\infty$ ) y para el flujo triangular continuo inducido por ella. Tras recordar algunas definiciones y resultados básicos, enseñamos como un flujo triangular continuo permite deducir la existencia de un atractor para un conjunto de problemas límite. En particular, considerando ciertas propiedades sobre las soluciones de un problema $\dot{x}=f(t, x)$, conseguimos la existencia de un atractor pullback acotado para los procesos inducidos por sistemas cuyo campo vectorial esté en el conjunto alpha-límite, en el conjunto omega-límite o en la envolvente de $f$. Además, proporcionamos un resultado de existencia de un atractor pullback y un atractor global para el flujo triangular inducido.

También, facilitamos condiciones suficientes para aplicar los resultados anteriores. En concreto, a través de resultados de comparación, obtenemos varios atractores para ambos, proceso y flujo triangular inducidos. Comparamos el tamaño de las soluciones de un sistema diferencial de Carathéodory, en primer lugar con el tamaño de las soluciones de una ecuación lineal escalar de Carathéodory adecuada y después con el tamaño de las soluciones de un sistema lineal de ecuaciones de Carathéodory. Finalizamos el capítulo aplicando los resultados obtenidos al ejemplo del sistema compartimental de la sección 3.4.3, y de este modo, obtener condiciones suficientes para la existencia de distintos tipos de atractores para los procesos y flujos triangulares inducidos.

\section{Continuidad del semiflujo para DDEs de Carathéodory}

En el capítulo final de la memoria, mostramos como la teoría desarrollada para ecuaciones diferenciales ordinarias de Carathéodory puede extenderse a ecuaciones diferenciales de Carathéodory con retardo constante del tipo (S.3).

Comenzamos el capítulo con algunos resultados preliminares sobre ecuaciones diferenciales de Carathéodory con retardo constante y, a continuación, introducimos nuevas topologías de tipo híbrido respecto de las que presentamos en el Capítulo 2. El término híbrido quiere enfatizar el hecho de que, a pesar de seguir pidiendo una convergencia de tipo $L_{l o c}^{1}$, trataremos las primeras $N$ variables espaciales (que representan el estado actual del sistema en una DDE) de manera diferente respecto a las últimas $N$ (que representan la historia del estado). Además, relacionamos estas topologías con las introducidas en el Capitúlo 2 y 
enseñamos como aplicar o desarrollar, en este nuevo contexto, algunos de los resultados topológicos obtenidos anteriormente en la memoria.

Finalmente, probamos la continuidad del semiflujo triangular inducido por problemas del tipo (S.3), cuando $\mathfrak{L} \mathfrak{C}$ está dotado de una de las topologías $\mathcal{T}_{B}, \mathcal{T}_{D}$ del Capítulo 2, o una de las nuevas topologías hibridas (y $f$ satisfaga hipótesis adecuadas). Por consiguiente, la teoría queda lista para poder desarrollar, donde sea posible, resultados análogos a los contenidos en los Capitúlos 3 y 4, pero para ecuaciones diferenciales de Carathéodory con retardo constante.

\section{Conclusiones}

La teoría desarrollada en esta memoria permite extender el formalismo de flujo y semiflujo triangular a las ecuaciones diferenciales ordinarias y con retardo constante de tipo Carathéodory, utilizando topologías métricas (fuertes y débiles) de tipo integral. Como consecuencia, el estudio del comportamiento cualitativo de las soluciones de esta clase de problemas podrá llevarse a cabo mediante el uso de herramientas de dinámica topológica.

Este trabajo incluye diversas aplicaciones para ecuaciones diferenciales ordinarias de Carathéodory como el estudio de flujos triangulares linealizados, la propagación de la dicotomía exponencial y del espectro dicotómico de un sistema lineal y el estudio de los atractores pullback y globales, así como algunos ejemplos inspirados por modelos de fenómenos reales que demuestran la aplicabilidad de la teoría desarrollada.

Además la tesis proporciona una descripción detallada de la estructura topológica considerada en los espacios de funciones de Carathéodory estudiados, muchos de ellos introducidos por primera vez. Se caracterizan las clases de equivalencia de las funciones que definen las ecuaciones, se estudia la propagación de las $m$-cotas y l-cotas a través de los límites en las topologías dadas y se obtienen caracterizaciones de los subconjuntos relativamente compactos.

Por último, la construcción y caracterización de los distintos semiflujos continuos para ecuaciones funcionales de Carathéodory con retardo constante, prepara el camino para el estudio posterior del comportamiento cualitativo de las soluciones y de la existencia de atractores pullback y globales que será llevado a cabo en futuras publicaciones.

La teoría presentada aporta ideas para investigaciones posteriores, tanto en el caso de ecuaciones ordinarias como en el de ecuaciones funcionales con retardo constante. Entre las posibles direcciones para el futuro queremos señalar las aplicaciones a teoría de bifurcación, teoría de control, biología matemática y análisis numérico. 



\section{Bibliography}

[1] L. Ambrosio, N. Fusco, D. Pallara: Functions Of Bounded Variation And Free Discontinuity Problems, Oxford Mathematical Monographs. The Clarendon Press Oxford University Press, New York, 2000.

[2] L. Arnold: Random Dynamical Systems, Springer-Verlag, Berlin, Heidelberg, 1998.

[3] Z. Artstein: Topological dynamics of an ordinary differential equation, $J$. Differential Equations 23 (1977), 216-223.

[4] Z. Artstein: Topological dynamics of ordinary differential equations and Kurzweil equations, J. Differential Equations 23 (1977), 224-243.

[5] Z. Artstein: The limiting equations of non-autonomous ordinary differential equations, J. Differential Equations 25 (1977), 184-202.

[6] B. Aulbach, T. Wanner: Integral manifolds for Carathéodory type differential equations in Banach spaces, Six Lectures on Dynamical Systems (B. Aulbach \& F. Colonius eds), World Scientific, Singapore, 1996, 45-119.

[7] M. Bebutov: On dynamical systems in the space of continuous functions, Boll. Moskov. Univ. Matematica (1941), 1-52.

[8] R. Bellman, J.M. Danskin: A survey of the mathematical theory of time-lag, retarded control, and hereditary processes, The Rand Corporation, R-256, 1954.

[9] A. Berger, S. Siegmund: On the gap between Random Dynamical Systems and Continuous Skew Products, J. Dynam. Differential Equations 15 (2003), 237-279.

[10] S.I. Bodin, R.J. SACKER: A new approach to asymptotic diagonalization of linear differential systems, J. Dynam. Differential Equations 12 (2000), $229-245$.

[11] A. Bressan, B. Piccoli: Introduction To The Mathematical Theory Of Control, AIMS on Applied Math. vol. 2, American Institute of Mathematical Sciences (AIMS), Springfield, MO, 2007. 
[12] B. Brogliato: Nonsmooth Mechanics Models, Dynamics And Control, Third Edition, Communications and Control Engineering Series, Springer, Switzerland, 2016.

[13] T. Caraballo, X. Han: Applied Non-Autonomous And Random Dynamical Systems. Applied Dynamical Systems. SpringerBriefs in Mathematics. Springer, Cham, 2016.

[14] T. Caraballo, J.A Langa, R. Obaya: Pullback, forward and chaotic dynamics in $1 \mathrm{D}$ noautonomous linear-dissipative equations, Nonlinearity 30 (2017), no. 1, 274-299.

[15] C. CARAthéodory: Vorlesungen Über Reelle Funktionen, Springer Fachmedien Wiesbaden GMBH, Leipzig, 1927.

[16] A. Carvalho, J.A. Langa, J. Robinson: Attractors for infinitedimensional non-autonomous dynamical systems, Springer-Verlag New York, 2013.

[17] R.E. Castillo, H. Rafeiro: An Introductory Course In Lebesgue Spaces, Springer, Switzerland, 2016.

[18] D.N. Cheban, P. Kloeden, B. Schmalfuss, B: The relationship between pullback, forward and global attractors of non-autonomous dynamical systems, Nonlinear Dyn. Syst. Theory 2 (2002), no. 2, 125-144.

[19] F. Clarke: Discontinuous feedback and nonlinear systems, IFAC Proceedings Volumes 43 (2010), 1-29.

[20] S.-N. Chow, H. LeIva: Dynamical spectrum for time dependent linear systems in Banach spaces, Japan J. Indust. Appl. Math. 11 (1994), 379-415.

[21] E.A. Coddington, N. Levinson: Theory Of Ordinary Differential Equations, McGraw-Hill, New York, 1955.

[22] M.A. Cruz, J.K. Hale: Existence, uniqueness and continuous dependence for hereditary systems, Ann. Math. Pura Appl. 85 (1970), 63-82.

[23] L. Dieci, C. Elia, D. Pi: Limit cycles for regularized discontinuous dynamical systems with a hyperplane of discontinuity., Discrete Contin. Dyn. Syst. Ser. B 22 (2017), 3091-3112.

[24] N. Dunford, J.T. Schwartz: Linear Operators: Part I General Theory, Wiley-Interscience, New York, 1988.

[25] R. Fabbri, R.A. Johnson, P.E. Kloeden: Digitization of nonautonomous control systems, J. Differential Equations 195 (2003), 210-229. 
[26] A.F. Filippov: Differential Equations With Discontinuous Righthand Sides, Math. Appl., Kluwer Academic Publishers, Dordrecht, the Netherlands, 1988.

[27] L. GRÜnE: Asymptotic Behavior of Dynamical and Control Systems under Perturbation and Discretization. Springer Lecture Notes in Mathematics, Vol. 1783, Springer, Berlin, 2002.

[28] W.M. Haddad, V. Chellaboina, Q. Hui: Nonnegative And Compartmental Dynamical Systems, Princeton University Press, Princeton, Oxford, 2010.

[29] J.K. Hale, S.M. Verduyn Lunel: Introduction To Functional Differential Equations, Applied Mathematical Sciences, 99, Springer-Verlag, NewYork, 1993.

[30] A.J. Heunis: Continuous dependence of the solutions of an ordinary differential equation, J. Differential Equations 54 (1984), 121-138.

[31] E. Hewitt, K. Stromberg: Real And Abstract Analysis. A Modern Treatment Of The Theory Of Functions Of A Real Variable, Springer-Verlag, New York, 1975.

[32] J.A. JACQuez: Compartmental Analysis In Biology And Medicine, Third Edition, Thomson-Shore Inc., Ann Arbor, Michigan, 1996.

[33] J.A. JACQUEZ, C.P. Simon: Qualitative theory of compartmental systems, SIAM Review 35 No.1 (1993), 43-79.

[34] R. Johnson, R. Obaya, S. Novo, C. Nuñez, R. Fabbri: Nonautonomous Linear Hamiltonian Systems: Oscillation, Spectral Theory And Control., Developments in Mathematics 36, Springer, Switzerland, 2016.

[35] O. Kallenberg: Random Measures, Third Edition, Akademie-Verlag, Berlin, 1983.

[36] P. Kloeden, M. Rasmussen: Nonautonomous Dynamical Systems, Mathematical Surveys and Monographs, 176, American Mathematical Society, Providence, RI, 2011.

[37] J. KurzweIL: Ordinary Differential Equations, Studies in Applied Mechanics 13, Elsevier, Amsterdam, 1986.

[38] I.P. Longo, S. Novo, R. OBaya: Topologies of $L_{l o c}^{p}$ type for Carathéodory functions with applications in non-autonomous differential equations, J. Differential Equations 263 (2017), 7187-7220. 
[39] I.P. Longo, S. Novo, R. OBaya: Weak topologies for Carathéodory differential equations. Continuous dependence, exponential dichotomy and attractors, submitted. arXiv:1709.06343v2 [math.DS]

[40] D. Nešić, A.R. Teel, P.V. Kokotović: Sufficient conditions for stability of sampled-data nonlinear systems via discrete time approximations, System Control Newslett. 38 (1999), 259-270.

[41] R.K. Miller, G. Sell: Volterra Integral Equations and Topological Dynamics, Mem. Amer. Math. Soc., no. 102, Amer. Math. Soc., Providence, 1970.

[42] R.K. Miller, G. Sell: Existence, uniqueness and continuity of solutions of integral equations, Ann. Math. Pura Appl. 80 (1968), 135-152; Addendum: ibid. 87 (1970), 281-286.

[43] L.W. Neustadt: On the solutions of certain integral-like operator equations. Existence, uniqueness and dependence theorems, Arch. Rational Mech. Anal. 38 (1970), 131-160.

[44] C. Olech, Z. Opial: Sur une inégalité différentielle, Ann. Polon. Math. 7 (1960) 247-254.

[45] Z. OPIAL: Continuous parameter dependence in linear systems of differential equations, J. Differential Equations 3 (1967), 571-579.

[46] P. Osinenko, G. Devadze, S. Streif: Analysis of the Carathéodory's theorem on dynamical system trajectories under numerical uncertainty, IEEE/CAA Journal of Automatica Sinica 5 (2018), 787-793.

[47] K. Popp, P. Stelter: Stick-slip vibrations and chaos, Philos. Trans. Roy. Soc. A 332 (1990), 89-105.

[48] C. Pötzsche: Persistence and imperfection of nonautonomous bifurcation patterns, J. Differential Equations 250 (2011), 3874-3906.

[49] C. Pötzsche, M. Rasmussen: Computation of integral manifolds for Carathéodory differential equations, J. Numer. Anal. 30 (2010), 401-430.

[50] M. Rasmussen, A. Hastings, M.J. Smith, F.B. Agusto, B.M.ChenCharpentier, F.M. Hoffman, J. Jiang, K.E.O.Todd-Brown, Y. WAng, Y.P. WAng, Y. LuO: Transit times and mean ages for nonautonomous and autonomous compartmental systems, J. Math. Biol. 73 (2016), 1379-1398.

[51] R.J. SACKer, G.R. SELl: A spectral theory for linear differential systems, J. Differential Equations 27 (1978), 320-358. 
[52] G. SELL: Compact sets of nonlinear operators, Funkcial. Ekvac. 11 (1968), $131-138$.

[53] G. SELL: Topological Dynamics and Ordinary Differential Equations, Van Nostrand-Reinhold, London, 1971.

[54] W. Shen, Y. Yi: Almost automorphic and almost periodic dynamics in skew-product semiflows, Mem. Amer. Math. Soc. 136, no. 647, Amer. Math. Soc., Providence, 1998.

[55] S. Siegmund: Dichotomy spectrum for non-autonomous differential equations, J. Dynam. Differential Equations 14 (2002), 243-258.

[56] H. Smith: An Introduction To Delay Differential Equations With Applications To The Life Sciences, Springer, New York, 2011. 